\title{
Hydrogeochemical Data for Thermal and Nonthermal Waters and Gases of the Valles Caldera- Southern Jemez Mountains Region, New Mexico
}

\author{
Lisa Shevenell \\ Fraser Goff \\ François Vuataz* \\ P. E. Trujillo, Jr.
}

Dale Counce

Cathy J. Janik**

William Evans ${ }^{\star \star}$

\section{DISCLAIMER}

This report was prepared as an account of work sponsored by an agency of the United States Government. Neither the United States Government nor any agency of the United States employees, makes any warranty, express or implied, or assumes any legal liability or responsiprocess disclosed, or completeness, or usefulness of any information, apparatus, product, or ence herein to any specific commercial use would not infringe privately owned rights. Refermanufacturer, or otherwise doescial product, process, or service by trade name, trademark, mendation, or favoring by the not necessarily constitute or imply its endorsement, recomand opinions of authors expressed herein Government or any agency thereof. The views United States Government or any agency do not necessarily state or reflect those of the 



\section{DISCLAIMER}

This report was prepared as an account of work sponsored by an agency of the United States Government. Neither the United States Government nor any agency Thereof, nor any of their employees, makes any warranty, express or implied, or assumes any legal liability or responsibility for the accuracy, completeness, or usefulness of any information, apparatus, product, or process disclosed, or represents that its use would not infringe privately owned rights. Reference herein to any specific commercial product, process, or service by trade name, trademark, manufacturer, or otherwise does not necessarily constitute or imply its endorsement, recommendation, or favoring by the United States Government or any agency thereof. The views and opinions of authors expressed herein do not necessarily state or reflect those of the United States Government or any agency thereof. 


\section{DISCLAIMER}

Portions of this document may be illegible in electronic image products. Images are produced from the best available original document. 
HYDROGEOCHEMICAL DATA FOR THERMAL AND NONTHERMAL WATERS AND GASES OF THE VALLES CALDERA--SOUTHERN JEMEZ MOUNTAINS REGION, NEW MEXICO

by

Lisa Shevenell, Fraser Goff, François Vuataz,

P. E. Trujillo, Jr., Dale Counce, Cathy J. Janik, and William Evans

\section{ABSTRACT}

This report presents field, chemical, gas, and isotopic data for thermal and nonthermal waters of the southern Jemez Mountains, New Mexico. This region includes all thermal and mineral waters associated with Valles Caldera and many of those located near the Nacimiento Uplift, north of San Ysidro. Waters of the region can be categorized into five general types: (1) surface and near-surface meteoric waters; (2) acid-sulfate waters at Sulphur Springs (Valles Caldera); (3) thermal meteoric waters in the ring fracture zone (Valles Caldera); (4) deep geothermal waters of the Baca geothermal field and derivative waters in the Soda Dam and Jemez Springs area (Valles Caldera); and (5) mineralized waters near San Ysidro. Some waters display chemical and isotopic characteristics intermediate between the types 1isted. Data in this report will help in interpreting the geothermal potential of the Jemez Mountains region and will provide background for investigating problems in hydrology, structural geology, hydrothermal alterations, and hydrothermal solution chemistry.

I. INTRODUCTION

This report is a revised edition of an earlier data report, LA-9367OBES, Goff et al. (1982), and contains 107 additional listings including 9 analyses from wells in the Baca geothermal field. Other additions include tables of gas analyses and calculated temperatures from chemical geothermometers. The manner in which the samples are listed has been revised in this report; thermal and nonthermal waters are currently grouped according to their locations in the Jemez Mountains region. 
The Jemez Mountains consist of volcanic rocks of basaltic to rhyolitic composition that overlie Tertiary to Paleozoic sediments on the western margin of the Rio Grande rift. Volcanic activity culminated in the Pleistocene with eruption of $1600 \mathrm{~km}^{3}$ of Bandelier Tuff and with formation of the Valles Caldera, a large well-preserved silicic cauldron. The Valles region contains a variety of hot springs having distinct geologic, chemical, and isotopic characteristics. Young, large silicic volcanic centers such as the Valles Caldera have great potential for geothermal energy because they overlie shallow magma reservoirs of batholithic proportions. A study of the chemistry of geothermal fluids can provide information on the hydrothermal systems and the geologic formations and structures through which they flow. The purpose of this report is to present field, chemical, and isotopic data for cold waters, thermal waters, and gases in the Jemez Mountains area and to characterize different water types and relate them to the hydrothermat systems. These data are presented to aid the overall assessment of geothermal resources of the Jemez Mountains and to provide data for other scientific investigations.

Different aspects of the geohydrology and geochemistry of waters in the Jemez Mountains have been described by Goff and Grigsby (1982), Goff et al. (1981), Goff and Sayer (1980), Goff et al. (1985), Goff et al. (1982), Trainer and Lyford (1979), Trainer (1974, 1975, 1978, 1984), Titus (1961), Purtymun and Johansen (1974), Purtymun et al. (1974, 1980), Purtymun (1977), Phillips et a1. (1984), Vuataz and Goff (1986), White (1986), Grigsby et a1. (1984), and Truesdell and Janik (1986).

11. SIMPLIFIED GEOLOGY

The geology of the Jemez Mountains has been described by Ross et al. (1961), Smith et a1. (1961), Griggs (1964), Doell et al. (1968), Bailey et al. (1969), Laughl in (1981), Laughl in et al. (1983), Gardner and Goff (1984), Heiken and Goff (1983), Nielson and Hulen (1984), Gardner et al. (1986), Heiken et al. (1986), and Self et al. (1986). Smith, Bailey, and Ross (1970) published an excellent regional geologic map. The Jemez Mountains consist of an extensive pile of Tertiary and Quaternary lavas and tuffs ( $>13$ to 0.1 Myr) overlying Precambrian granite, gneiss, and schist and the Paleozoic to Mesozoic sedimentary sequence of the Colorado Plateau (Figs. 1 and 2). The main Paleozoic units include the Pennsylvanian Madera Limestone and Sandia Formation and the red sandstones, siltstones, and shales of the Permian Abo 


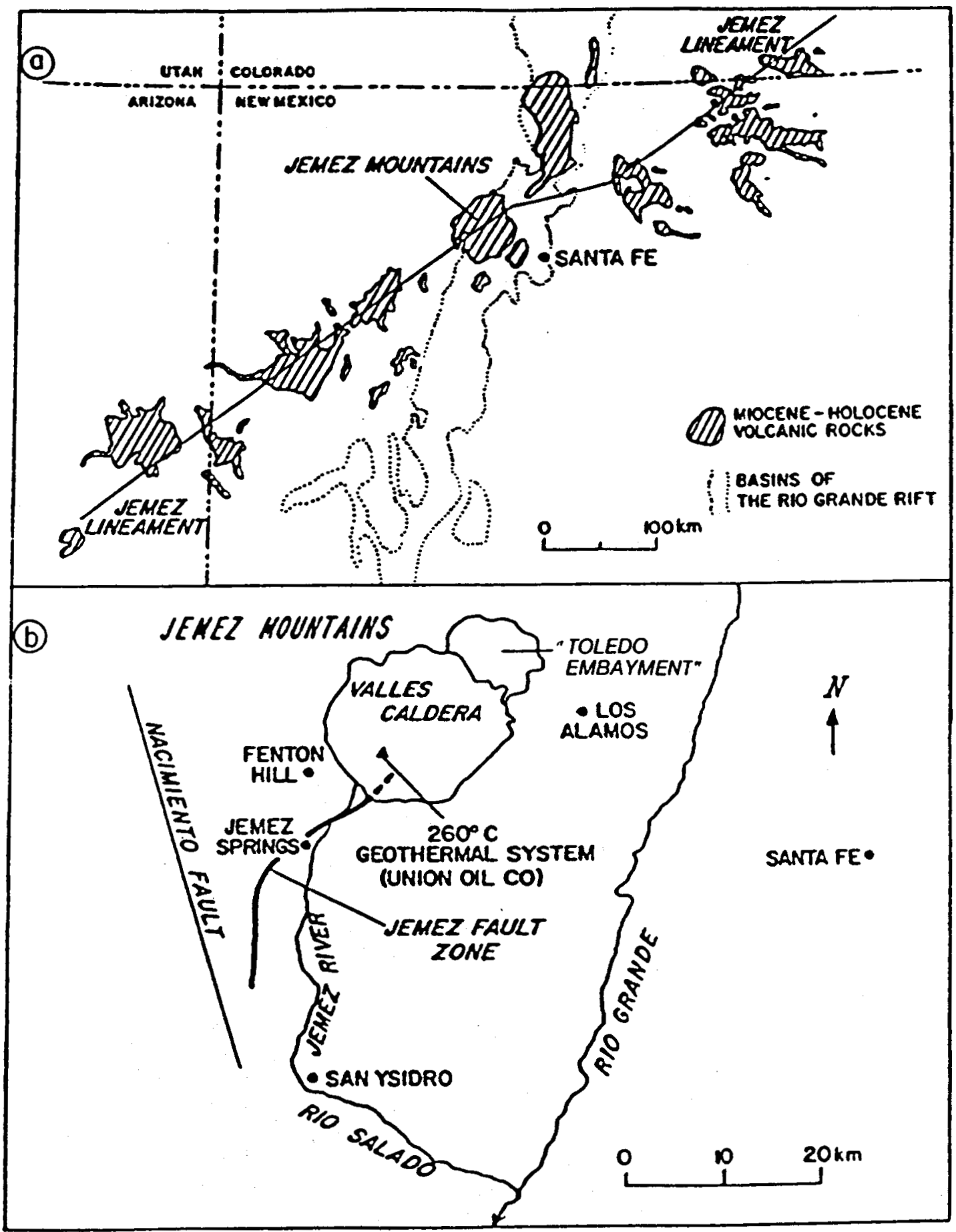

Fig. 1.

(a) Volcanic centers in relationship to the Rio Grande Rift and Jemez Lineament and (b) sketch of Jemez Mountains region. 


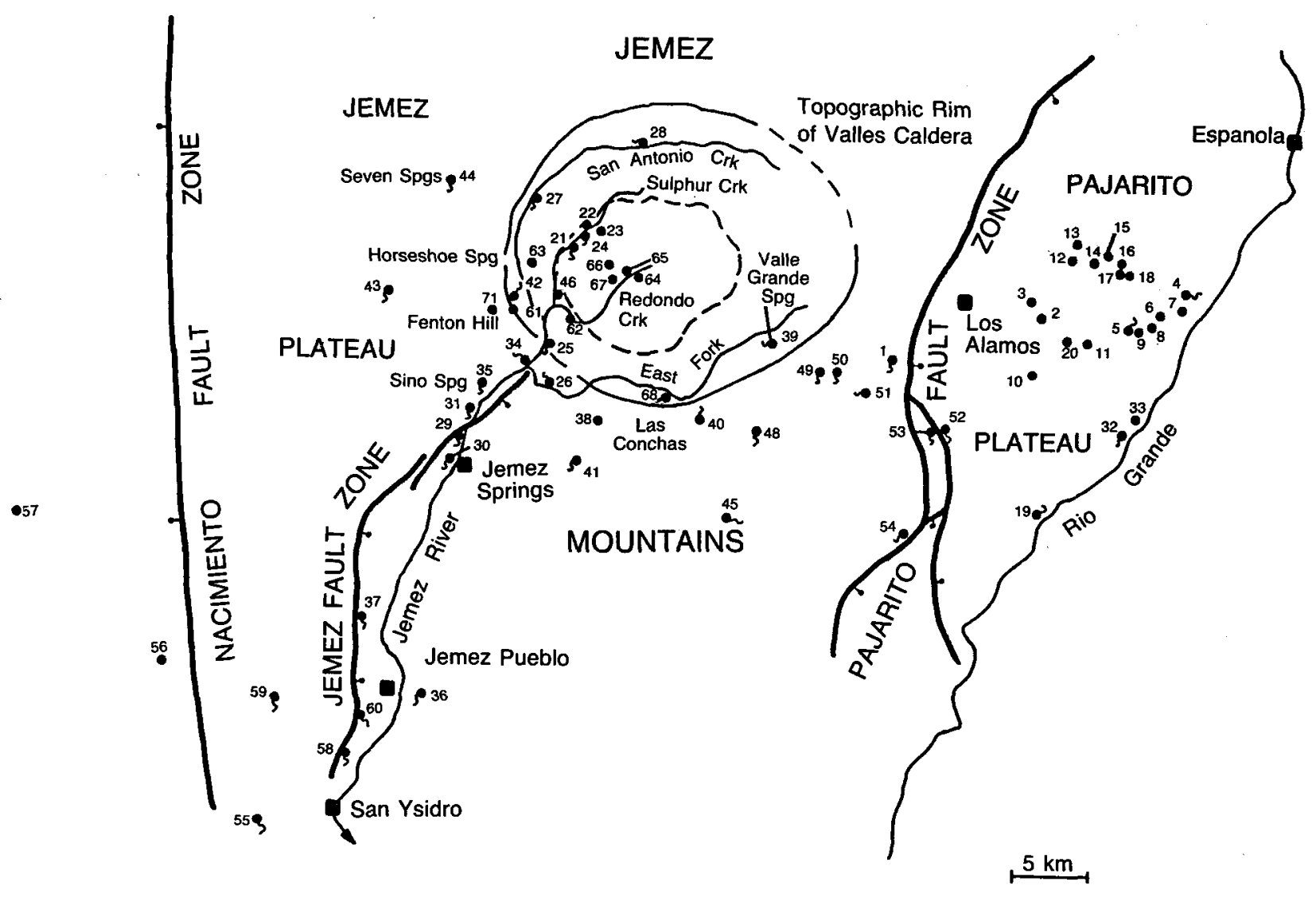

Fig. 2 .

Map of Jemez Mountains region showing major faults. Numbers refer to spring and well locations listed in tables in Appendix $B$.

and Yeso Formations. Mesozoic units include the Jurassic Entrada Formation and the Triassic Chinle Formation. Mesozoic outcrops are located south of the Jemez Mountains near San Ysidro. The older Mesozoic units include the Entrada Formation sandstone, the Jurassic Todilto Formation evaporites (mainly gypsum), and the Jurassic Morrison Formation shale and sandstone.

Colorado Plateau rocks of this region are down-faulted to the east into the Rio Grande rift. Unconsolidated Tertiary sediments of the Santa Fe Formation thicken eastward toward the axis of the rift. The Jemez Mountains volcanics occur at the intersection of the rift with the northeast-trending Jemez Lineament, a line of Miocene to Quaternary volcanic fields extending across the northwest portion of New Mexico (Aldrich and Laughl in 1984).

Volcanic activity commenced with dominantly mafic to intermediate lava flows that are partly interbedded with the Tertiary sediments. These rocks are best exposed in the areas north, northeast, and south of the valles 
Caldera complex. Two major eruptions of Bandelier rhyolite tuff in the early Pleistocene resulted in the formation of the Toledo and Valles Calderas. Deposits of tuff up to $300 \mathrm{~m}$ thick occur to the west and east of the caldera forming the Jemez and Pajarito Plateaus, respectively. The final activity in the Jemez Mountains involved eruption of rhyolite domes, obsidian, and tuffs in the moat zone of the Valles Caldera (Smith et al. 1970, Bailey et al. 1969, Gardner and Goff 1984).

\section{METHODS AND PROCEDURES FOR COLLECTION AND ANALYSIS OF WATERS}

Before 1982 temperatures were recorded with mercury thermometers, and field $\mathrm{pH}$ was determined using a $\mathrm{pH}$ meter or using sensitive, limited-range $\mathrm{pH}$ test papers (Colorfast Indicator Strips nos. 9581, 9582, and 9583). After 1982, $\mathrm{pH}$, temperature, conductivity, and Eh were measured in the field using a Presto-Tek model 500 digital recorder. Laboratory values of $\mathrm{pH}$ are not considered reliable because most waters gain or lose $\mathrm{CO}_{2}$ gas after sampling and before laboratory analysis. This gain or loss alters the concentration of bicarbonate ions, which in turn changes the $\mathrm{pH}$. Flow rates of springs were estimated visually or measured approximately with a bucket and stopwatch; flow rates of wells were obtained from measurements provided by well owners where possible. A compilation of photographs of thermal springs of the Jemez Mountains is presented in Appendix A (Figs. A-1 through A-11). Field data are recorded in Table $B-I$ (a 11 tables appear in Appendix $B$ ).

Samples of water for chemical analysis were filtered using a handoperated vacuum pump system or a large syringe attached to a filter holder containing $0.45-\mu \mathrm{m}$ filter paper. The filtered water was poured brimful into polyethylene bottles and sealed with polyseal caps. Five types of samples were collected: (1) a 500-me bottle of filtered unacidified water for anions, (2) a 250-me bottle of filtered acidified water for cations, (3) a 125-me bottle of filtered diluted water for silica, (4) a 500-me glass bottle of unfiltered water for tritium analysis, and (5) a 125-me glass bottle of unfiltered water for stable isotope analysis. The cation samples were acidified in the field so that the sample pH dropped below 2. Before 1982, the samples were acidified by the dropwise addition of dilute $\mathrm{HCl}$. After March 1982, 20 drops of $70 \% \mathrm{HNO}_{3}$ were used to acidify the samples in the less mineralized waters, and 40 drops were used in the highly mineralized waters. The bottles used for silica analyses contained $90 \mathrm{ml}$ of deionized water before 
$10 \mathrm{~m} \ell$ of sample were added. This dilution prevents polymerization of monomeric silica in more concentrated water samples before analysis. Samples for determination of $\mathrm{Al}$ were collected and analyzed according to our modified procedure of Barnes (1975). Major element analyses are presented in Table B-II.

Chemical analyses were performed by the following methods: $\mathrm{SiO}_{2}$ by a colorimetric method using ammonium molybdate; Al, As, Ba, Ca, Fe, K, Li, Mg, $\mathrm{Mn}$, Mo, $\mathrm{Na}$, Si, $\mathrm{Sr}$, and $\mathrm{Zn}$ by inductively coupled plasma (ICP) emission spectroscopy; $\mathrm{Ag}, \mathrm{Al}^{+3}, \mathrm{Cd}, \mathrm{Co}, \mathrm{Cr}, \mathrm{Cu}, \mathrm{Cs}, \mathrm{Ni}, \mathrm{Pb}$, and $\mathrm{Rb}$ by atomic absorption (AA) spectroscopy using a graphite furnace; $\mathrm{Br}, \mathrm{Cl}, \mathrm{NO}_{3}, \mathrm{PO}_{4}$, and $\mathrm{SO}_{4}$ by ion chromatography; $\mathrm{B}$ by colorimetry using azomethine- $\mathrm{H}$ or by ICP; $\mathrm{F}$ by selective ion electrode; low-level $\mathrm{Na}, \mathrm{K}$, and $\mathrm{Li}$ by flame atomic absorption; $\mathrm{HCO}_{3}{ }^{-}$and $\mathrm{CO}_{3}{ }^{-2}$ by titration with $\mathrm{H}_{2} \mathrm{SO}_{4} ; \mathrm{S}^{-2}, \mathrm{NH}_{4}$, and $\mathrm{O}_{2}$ (dissociated) with electrodes (Trujillo et al., in prep.). All chemical analyses were performed at the Fenton Hill Laboratory (LOS Alamos National Laboratory), and the data appear in Tables B-II and B-III.

Before 1982, oxygen-18 and deuterium analyses were performed by $L$. Merlivat of the Bureau des Isotopes Stables, Centre d'Etudes Nucléaires, Saclay, France; and after 1982, they were performed by Russ Harmon of the Stable Isotope Lab., Southern Methodist University, Dallas, Texas. Before 1982, tritium analyses were provided by Teledyne Isotopes, Westwood, New Jersey; after March 1982, samples were analyzed by H. Gote Ostlund, Tritium Laboratory at the University of Miami.

\section{GEOHYDROLOGY AND GEOCHEMISTRY}

The waters described in this report can be divided into several groups on the basis of field, chemical, and isotopic characteristics. In this section each group is discussed separately, but the reader should refer to Figs. 3-5 for sample locations and to Figs. 6-14 for chemical and isotopic differences and similarities among water types.

\section{A. Surface and Near-Surface Meteoric Water}

Surface and near-surface meteoric waters are generally cold, potable, and dilute. Waters included in this group issue from water-supply and test wells in the Pajarito Plateau (LA sample numbers) and from cold springs, creeks, and wells throughout the Valles Caldera-southern Jemez Mountains 


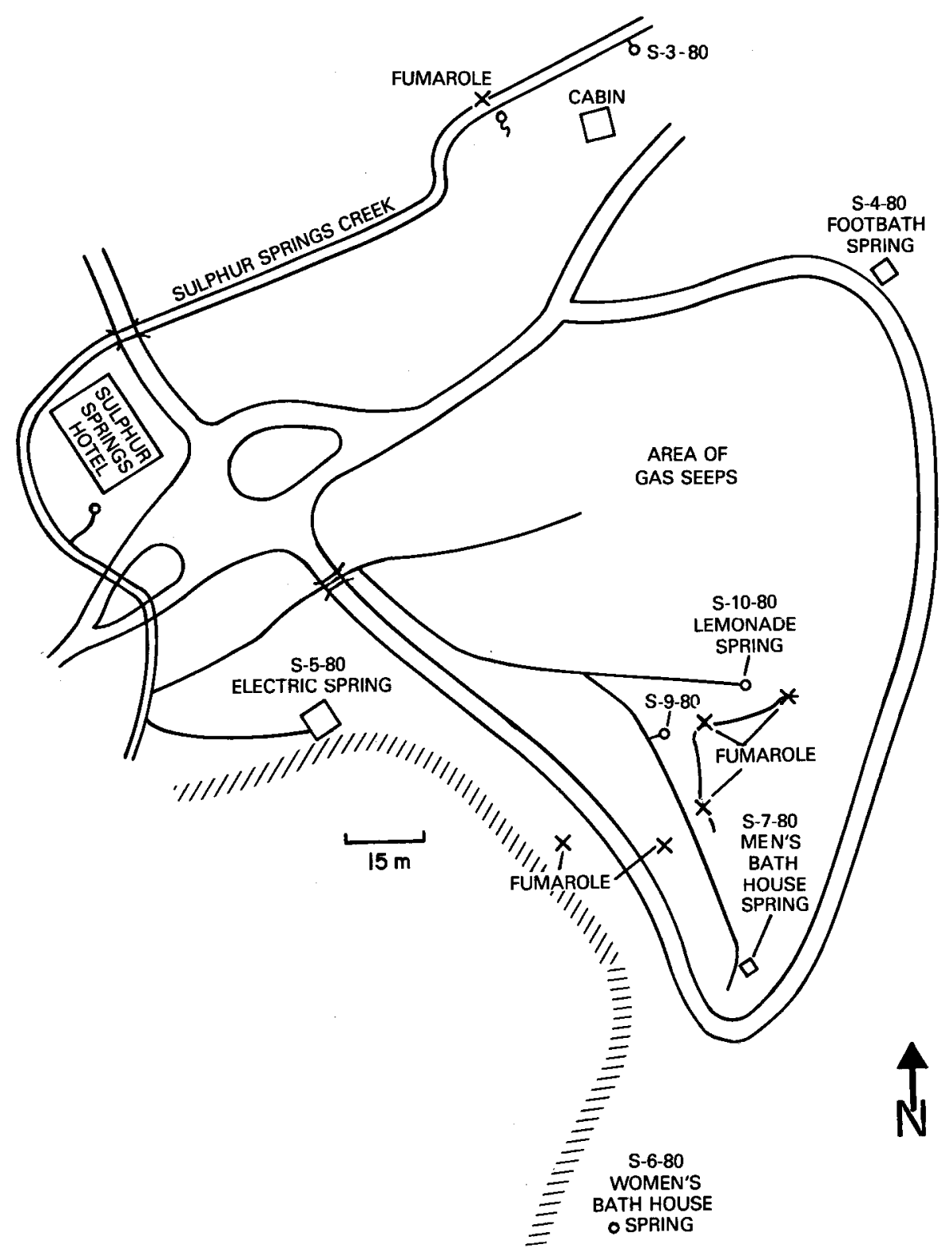

Fig. 3 .

Sketch map of Sulphur Springs area (modified from Summers 1976).

region (VA sample numbers; see Figs. 2-5 for Tocations). Cold meteoric waters occur in three different geologic settings: (1) within late Tertiary to Quaternary volcanic rocks of the Jemez Mountains volcanic field, (2) within late Tertiary basin-fill sediments of the Rio Grande rift, and (3) within Paleozoic to Mesozoic sediments of the Colorado Plateau. These three different settings produce subtle differences in the chemistry of near-surface waters.

The hydrology of the Pajarito Plateau has been described by Purtymun and Johansen (1974), and the geochemistry of the waters with respect to 


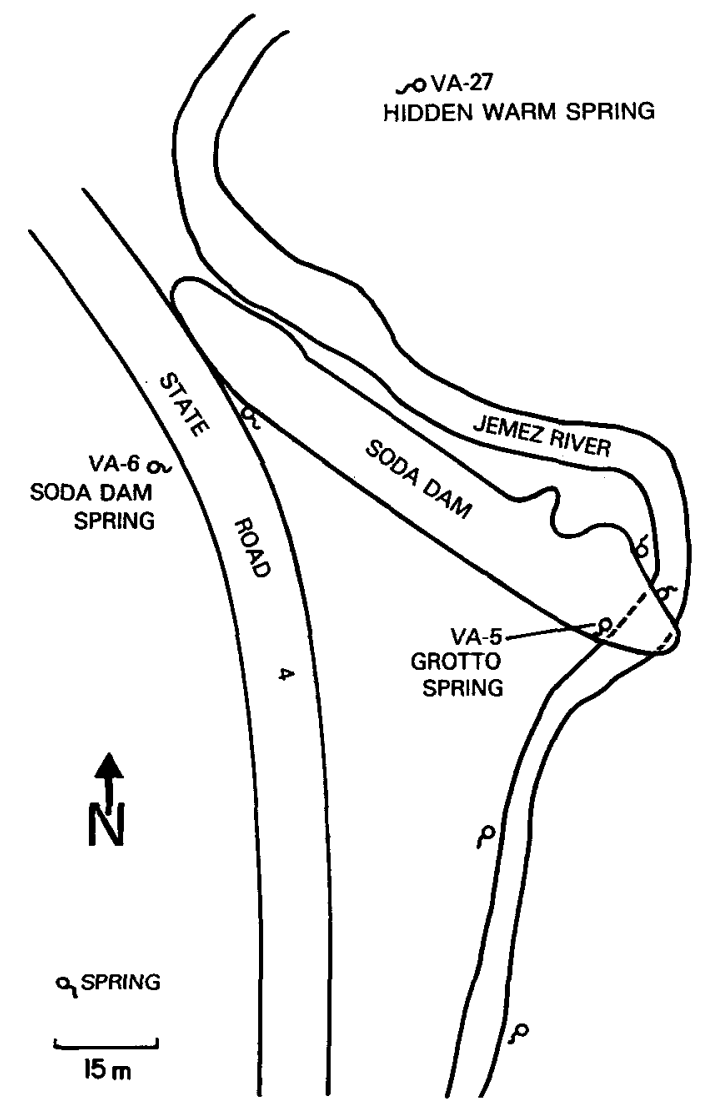

Fig. 4 .

Sketch map of Soda Dam area.

geothermal potential has been discussed in detail by Goff and Sayer (1980). Hydrology of the southern Jemez Mountains is not known in detail although the Jemez River and tributaries drain Valles Caldera in a southerly direction.

In general, surface and near-surface waters of this category are calcium-bicarbonate waters although some of the Los Alamos water-supply wells and cold mineral springs in the Valles Caldera region are sodium-bicarbonate waters (Fig. 6). The waters can be characterized by low conductivity (since they are relatively dilute), low temperature, and $\mathrm{pH}$ near 7 . They typically contain low concentrations of $\mathrm{F}, \mathrm{Cl}, \mathrm{Li}, \mathrm{B}$, and $\mathrm{SiO}_{2}$ (Figs. 7a through 11a) indicating a low-temperature, near-surface environment (White 1957). The wel1 waters of the Pajarito Plateau show a wider range in all these constituents than do most cold waters of the region, possibly because some waters have long resided in deep aquifers or have mixed with deep thermal/mineral water rising along faults in the Rio Grande rift (Goff and Sayer 1980). A few springs in the caldera region are anomalous in that they are somewhat mineralized but are 


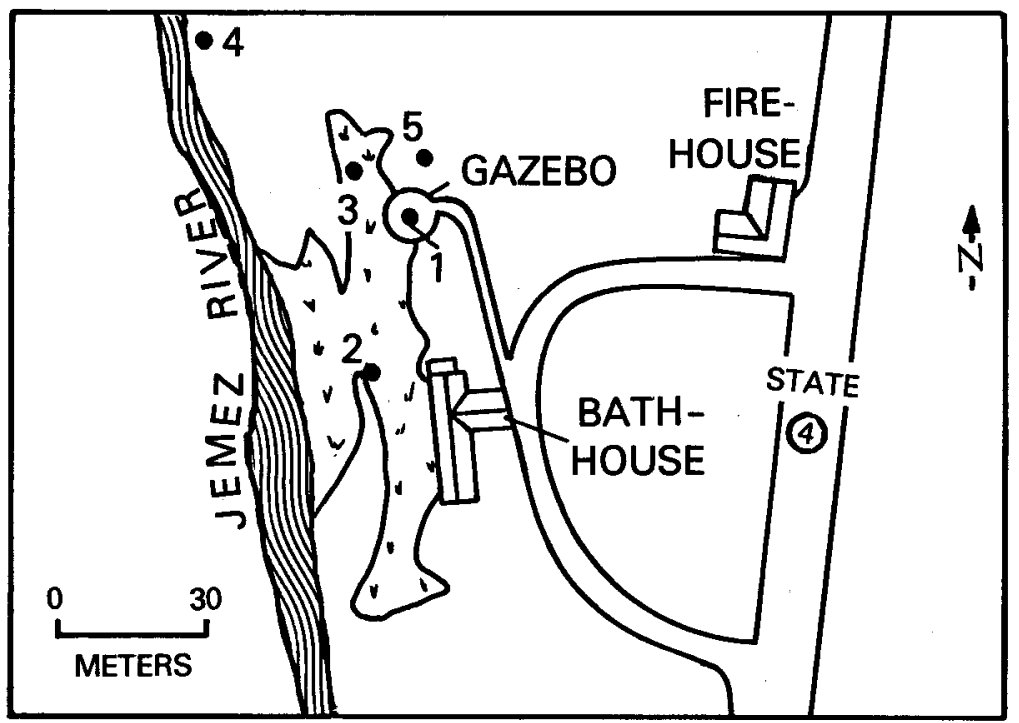

1. MAIN JEMEZ SPRING, VA-10

2. TRAVERTINE MOUND SPRING, VA-7

3. UNNAMED WARM SPRING, VA-12

4. BUDDHIST SPRING, VA-8

5. JEMEZ SPRINGS GEOTHERMAL WELL, VA-19

Fig. 5.

Sketch map of Jemez Springs area (modified from Summers 1976).

also cold (location nos. 34, 36, 37 and to a lesser extent location 31, Fig. 2; samples from Battleship Seep, Ponderosa Spring, Cañon Spring, and Panorama Spring, VA-39 and VA-40, Table B-II). These samples have much greater conductivity, comparatively high $\mathrm{Na}+\mathrm{K}$ relative to $\mathrm{Ca}$, high $\mathrm{Cl}$ relative to $\mathrm{HCO}_{3}$, and relatively high $F, B$, and Li (see Figs. 8a, 9a, 10a, 11a, and 14a). Two of the springs (location nos. 34 and 36 ) also have very low $\mathrm{SiO}_{2} \cdot$ These slightly mineralized meteoric waters issue from Mesozoic-Paleozoic sediments and either leach out evaporitic minerals from them or else contain an extremely small fraction of deep thermal water from Valles Caldera.

Trace elements in all waters in this group are fairly low (Table B-III). Strontium appears to be higher in waters issuing from Madera limestone (especially location nos. 34 and 36 ), suggesting that $\mathrm{Sr}$ is dissolved out of the carbonate rocks.

The tritium content of the surface waters in the Jemez Mountains is generally greater than 5 tritium units (T.U.), indicating that the waters are young, less than 50 years old (Table $B-I V)$. The $D$ and ${ }^{18} 0$ isotope ratios 


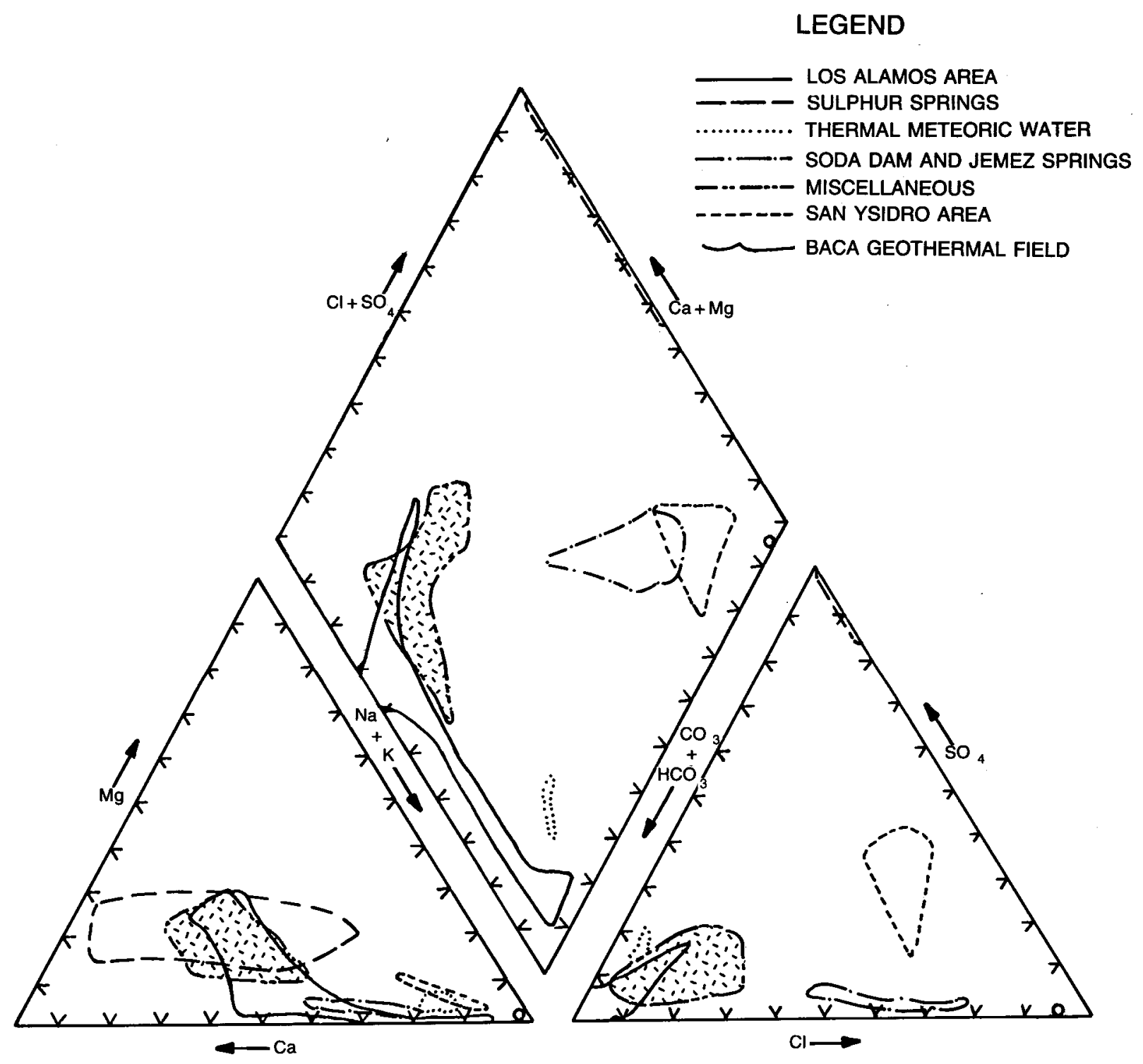

Fig. 6.

Piper diagram showing the range in chemical composition in equivalents of various types of water in southern Jemez Mountains, New Mexico. Patterns are added to help distinguish overlapping fields of data points.

(Fig. 13a) parallel the world meteoric water line (Craig 1961). The cold mineral waters described above are not isotopically distinct from other waters in this group.

B. Valles Caldera-Sulphur Springs Area

A suite of thermal and nonthermal acid-sulfate springs and mudpots and some associated dilute carbonated waters are located within the Valles Caldera near the western margin of the resurgent dome (Figs. 2 and 3 ). Discussion of 10 


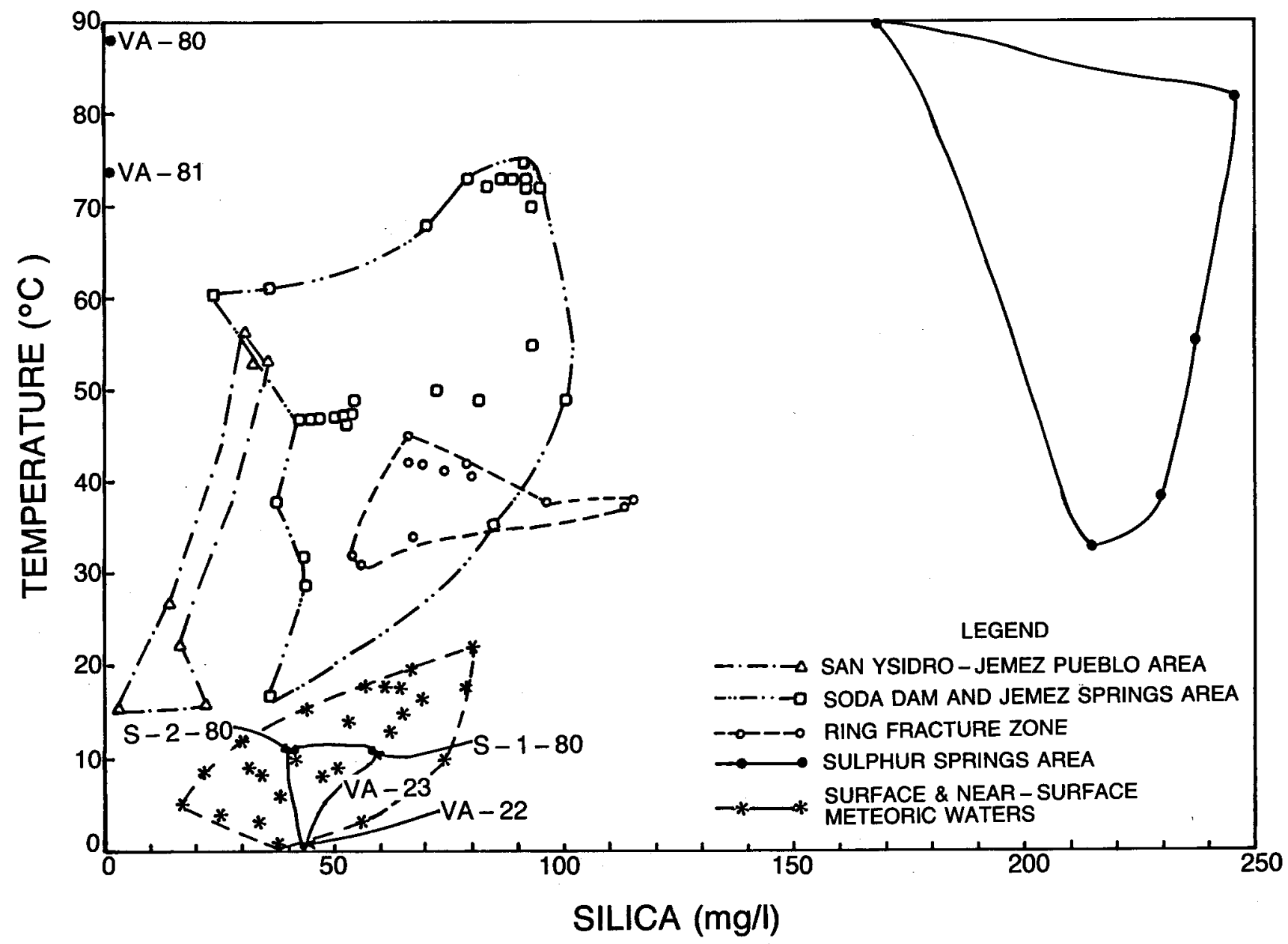

Fig. 7a.

Plot of $\mathrm{SiO}_{2}$ VS. measured temperature for various types of water in the southern Jemez Mountains region, New Mexico. Note that waters from Sulphur Springs area fall into two distinct fields.

the chemistry and origin of the Sulphur Springs has been presented by Trainer (1974), Goff and Grigsby (1982), and Goff et al. (1985). Names used for various springs in Tables B-I through B-VI correspond with those presented by Summers (1976). Rhyolitic flows and tuffs and caldera fill deposits in the area have been extensively altered and leached to clays, silica minerals, authigenic feldspars, and sulfates. Native sulfur, pyrite, and aluminum sulfates have precipitated near fumaroles (Charles et al. 1986). The waters are characterized by a high conductivity lexcept for the dilute bubbling waters) and low $\mathrm{pH}$, especially in the most concentrated waters. Most of the samples have moderate $F$ and low $B, L i$, and $C l$ (Figs. 8a through 11a). The $F$ concentrations are highest in the $\mathrm{Cl}$-free waters. $\mathrm{SiO}_{2}$ contents vary within 


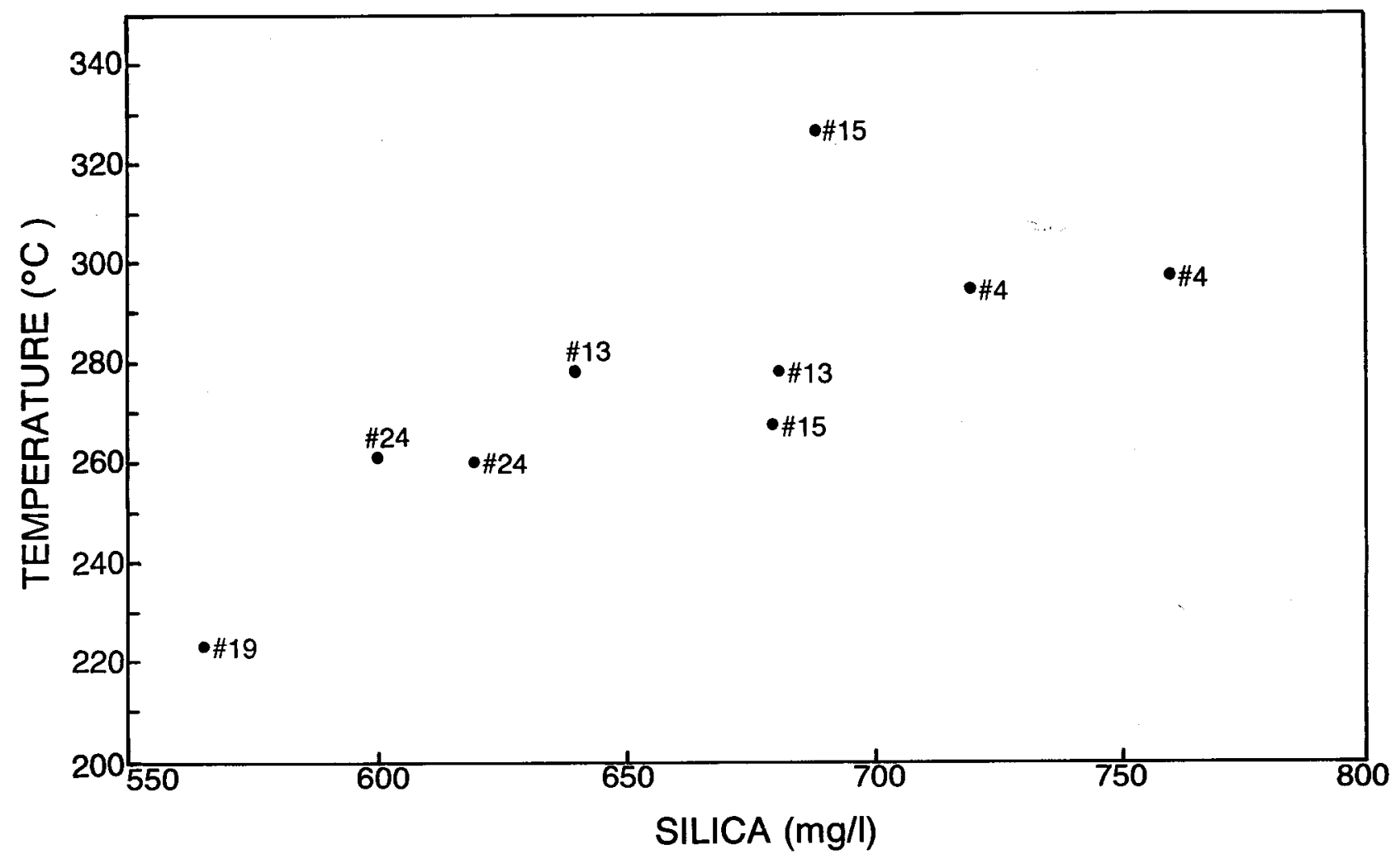

Fig. 7b.

Plot of $\mathrm{SiO}_{2}$ vs. temperature, which was calculated using steam-enthalpy tables, for the Baca geothermal wells. Numbers represent the well from which the sample was taken.

the suite, but the waters can generally be divided into two groups: a high-temperature, high-SiO 2 group and a low-temperature, low-SiO 2 group (Fig. 7a). This relationship is, in part, a function of the increasing solubility of $\mathrm{SiO}_{2}$ with increasing temperature.

Trace elements $\mathrm{Zn}, \mathrm{Cu}, \mathrm{Cr}$, $\mathrm{Co}$, and $\mathrm{Ni}$ show relatively high concentrations in acid waters that are also rich in Fe (Table B-III), a relationship probably due to the greater solubilities of these metals in acid waters.

The tritium contents of 2.1 and $2.3 \mathrm{~T}$.U. in the mudpot and steam at the Men's Bathhouse indicate that the condensed steam originated from a deep source of relatively great age, greater than 50 years old. However, Footbath Spring and the Women's Bathhouse Spring appear to be mixtures of condensed steam and near-surface waters having an age between 20 and 30 years. The $D$ and ${ }^{18} 0$ isotope ratios of the bubbling seep (sample VA-23, location no. 22 , Fig. 2) of the unnamed acid spring (sample VA-14, location no. 21, Fig. 2) fall on the world meteoric line, the unnamed spring being more enriched in ${ }^{18_{0}}$ 


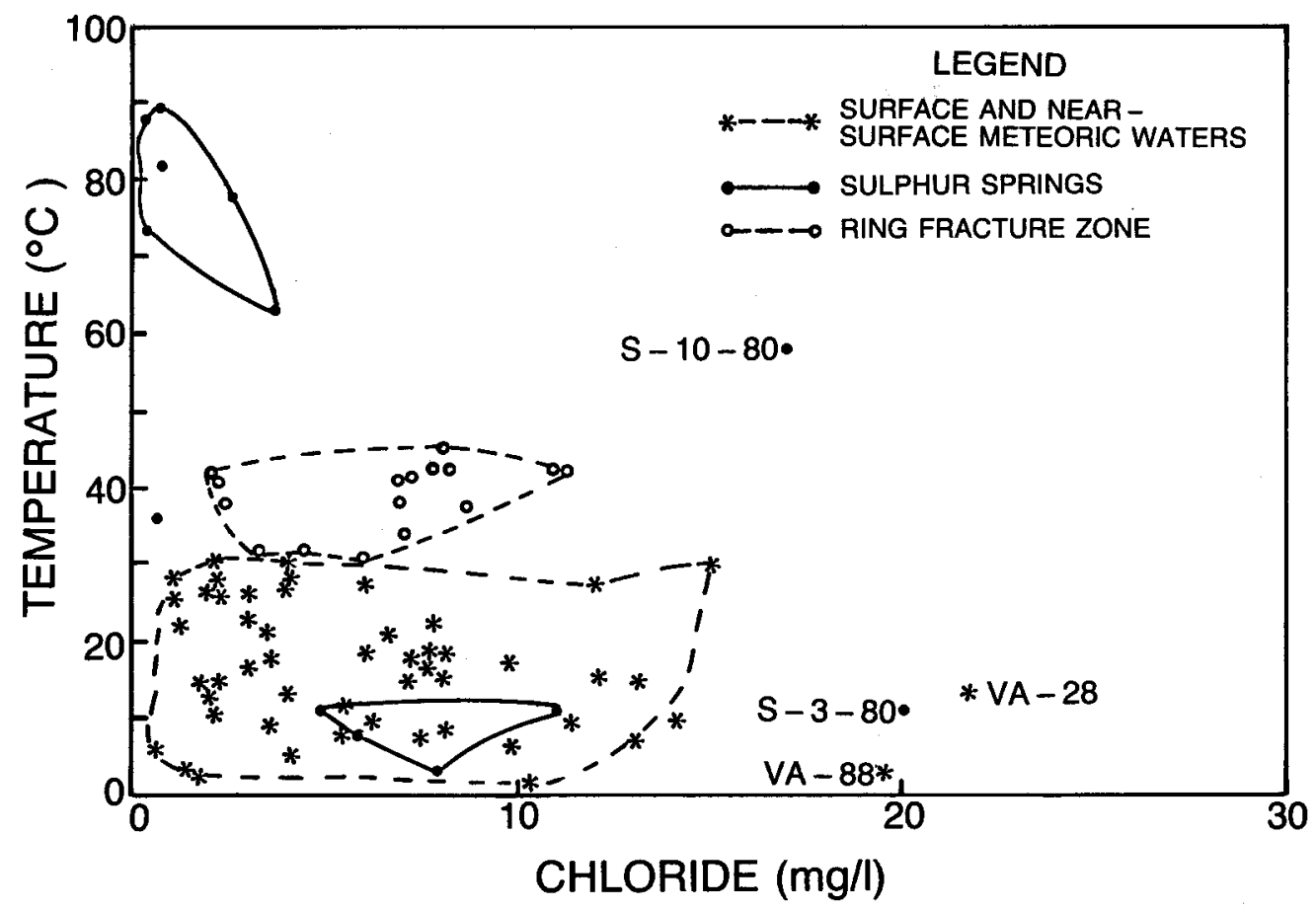

Fig. 8 a.

Plot of $\mathrm{Cl}$ vs. measured temperature for meteoric waters and waters in the ring fracture zone and Sulphur Springs areas in the southern Jemez Mountains region, New Mexico. Sample numbers refer to waters that fall outside the fields of generalized water types.

and D (Fig. 13b). In contrast, the Men's Bathhouse mudpot consists mostly of condensed steam from depth and is very enriched in ${ }^{18} 0$, probably the result of extensive surface evaporation.

The Sulphur Springs are typical of a vapor-dominated geothermal system (White et al. 1971) where water vapor, $\mathrm{H}_{2} \mathrm{~S}$, and $\mathrm{CO}_{2}$ rise from an underlying boiling water table. The known occurrence of a high-temperature $\left(280^{\circ} \mathrm{C}\right)$ hydrothermal system beneath the resurgent dome of Valles Caldera (Goff et al. 1985) indicates that such a boiling water table may exist beneath Sulphur Springs. Condensation of the steam near the surface and surface oxidation of $\mathrm{H}_{2} \mathrm{~S}$ to $\mathrm{H}_{2} \mathrm{SO}_{4}$ results in acid-sulfate water. The flowing springs mix seasonally with surface meteoric water since they show lower flow rates and higher temperatures during the dry months of the year.

\section{Valles Caldera-Ring Fracture Zone}

The thermal meteoric waters of this zone consist of a group of warm springs in the western ring fracture zone, previously described by Goff and Grigsby (1982), Goff and Sayer (1980), and Trainer (1974). The waters are 


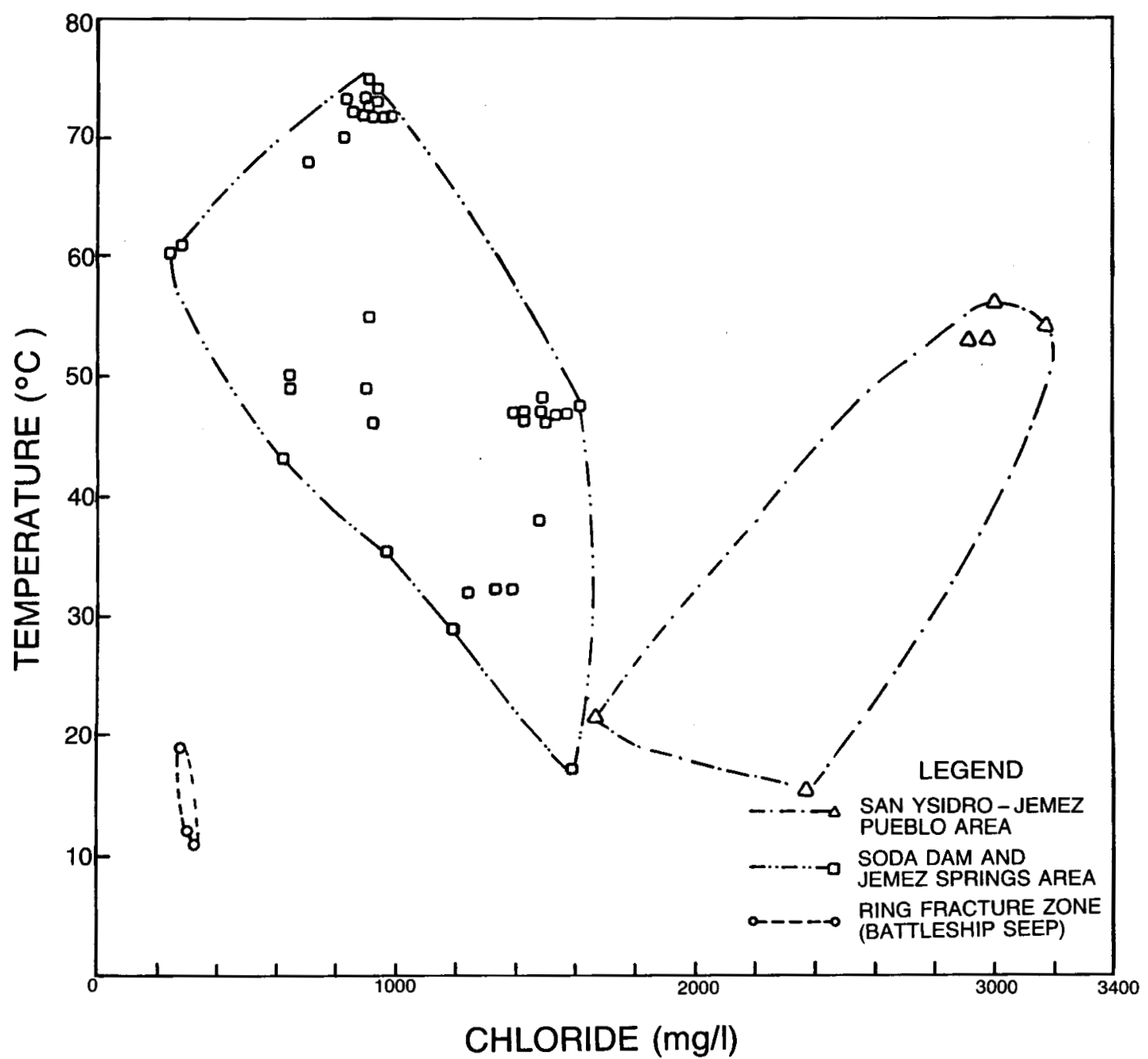

Fig. 8b.

Plot of $\mathrm{Cl}$ vs. temperature for waters in the San Ysidro and Soda Dam-Jemez Springs areas.

typically dilute, near neutral in $\mathrm{pH}$, and moderate in temperature $\left(31-45^{\circ} \mathrm{C}\right)$. They are sodium-bicarbonate waters displaying low concentrations of $\mathrm{F}, \mathrm{Cl}, \mathrm{B}$, and $L i$ (Figs. 8a, 9a, 10a, and 11a). The F, B, and $L i$ contents are slightiy higher than most of the surface waters in the caldera, and B and Li concentrations are both slightly enriched in samples with higher $\mathrm{Cl}$. McCauley Spring differs from the others in that it contains more $\mathrm{Ca}$ and $\mathrm{Mg}$ and less alkalis. A1l samples show low concentrations of the trace elements analyzed (Table $B-I I I)$. The $D$ and ${ }^{18} 0$ isotopes fall close to the world meteoric line in a tight cluster (Fig. 13b). The generally low tritium content $(0.2$ to 2.3 T.U.), but low trace element content, of these waters indicates that they are 


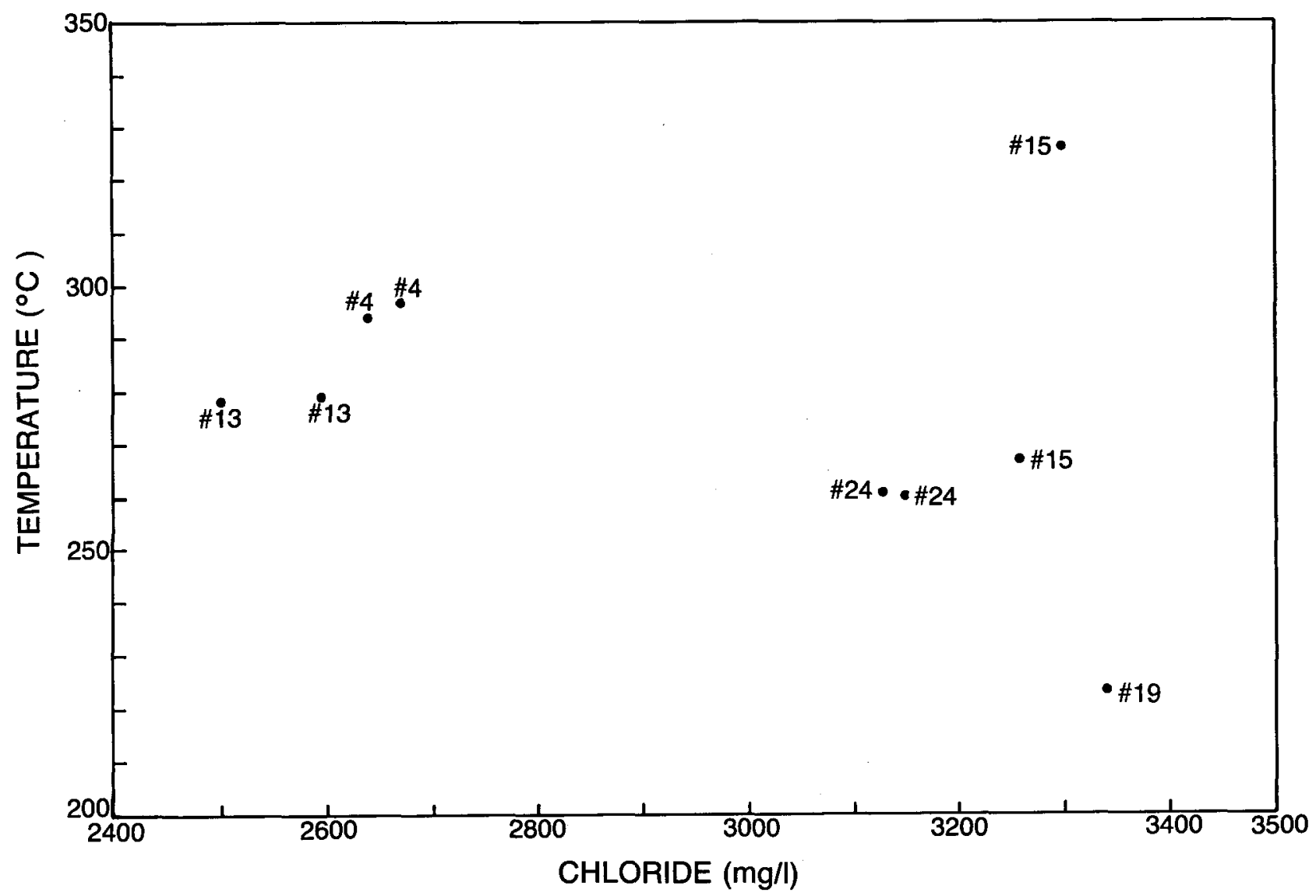

Fig. 8c.

Plot of $\mathrm{Cl}$ vs. temperature for the Baca geothermal wells. Temperatures were calculated using steam-enthalpy tables. Numbers refer to the well from which the sample was collected.

derived from relatively old groundwater circulating in the moat zone of the caldera.

These waters can be considered to be meteoric waters that have been heated during circulation in the ring fracture zone of the Valles Caldera. All waters of this type discharge near the youngest (less than 0.5 Myr) of the moat rhyolites erupted in the caldera. They are slightly depleted in $\mathrm{Ca}$ and Mg compared with surface waters in the caldera region because of the inverse relationship between carbonate solubility and temperature. Very slight mixing with deep thermal waters may have occurred in Spence, Little Spence, and possibly McCauley Hot Springs (location nos. 25 and 26, Fig. 2) because they have higher $\mathrm{Li}$ and $\mathrm{Na}$ than most of the meteoric waters in the Valles Caldera region. 


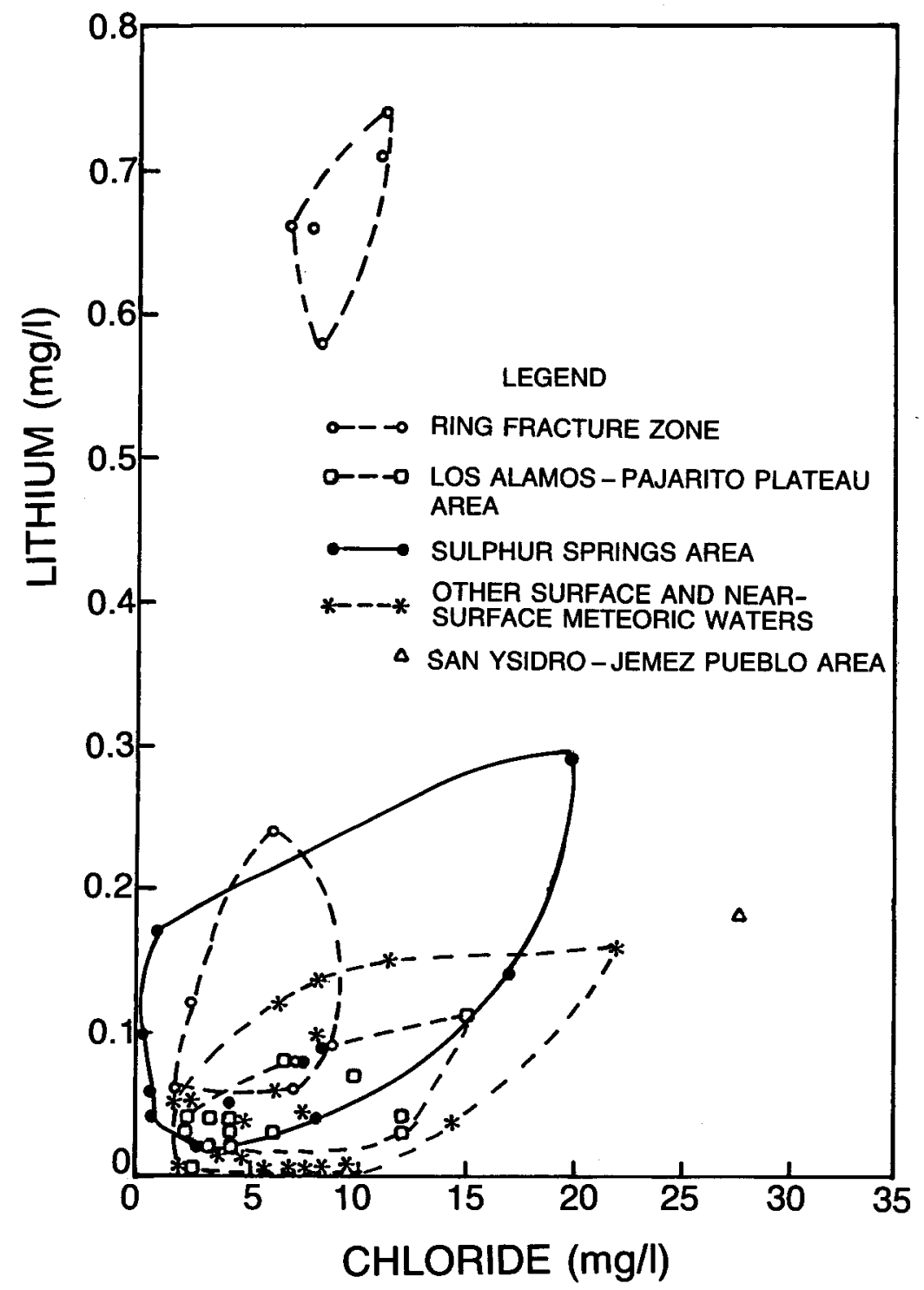

Fig. 9 a.

Plot of $\mathrm{Li}$ vs. $\mathrm{Cl}$ for waters with $\mathrm{Cl}$ concentrations of 1 ess than $50 \mathrm{mg} / \ell$ in the southern Jemez Mountains region, New Mexico.

\section{Baca Geothermal Field}

Several geothermal wells were drilled by Union 0il Company of California in the Redondo Creek area in the keystone graben of the resurgent dome. These wells, drilled on the Baca Location \#1, intersect the deepest and highest temperature fluids encountered in the Valles Caldera area. The chemistry of these well waters has been discussed by White et al. (1984), Truesdell and Janik (1986), and white (1986).

The Baca well waters are high-temperature, near-neutral to slightly alkaline, sodium-chloride fluids; the highest temperature measured was 


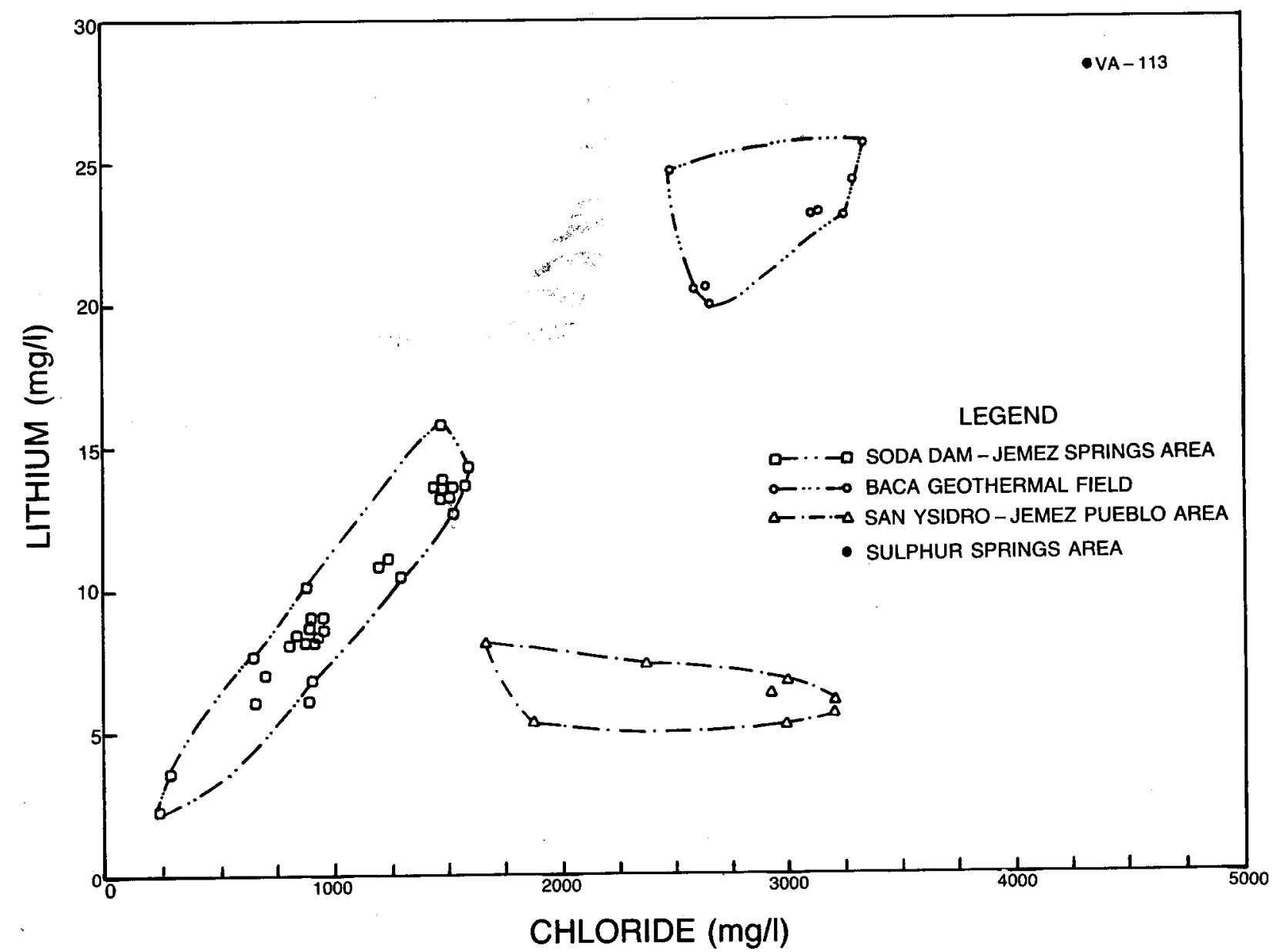

Fig. 9b.

Plot of $\mathrm{Li}$ vs. $\mathrm{Cl}$ for waters with $\mathrm{Cl}$ concentrations greater than $50 \mathrm{mg} / \mathrm{l}$ in the southern Jemez Mountains region, New Mexico. Sample VA-116, from the GRI wel1, plots out of the field of view. The sample number refers to waters that fall outside fields of generalized water types.

$297^{\circ} \mathrm{C}$ at Baca well \#4. They contain large amounts of $\mathrm{B}, \mathrm{Li}$, and $\mathrm{SiO}_{2}$ and relatively low concentrations of $\mathrm{HCO}_{3}, \mathrm{Ca}$, and $\mathrm{Mg}$ (Figs. 7b, 8bc, 9b, and 10b, Table B-II). These concentrations are typical of fluids in equilibrium with intermediate to silicic volcanic reservoir rocks, such as Bandelier Tuff (White et a1. 1984).

other trace element concentrations are generally low; As is the notable exception. Up to $5 \mathrm{mg} / \mathrm{l}$ of As has been detected in Baca Well \#24 (Table B-III).

Tritium values of the Baca wells are all less than 1.1 T.U. (Table B-IV). This low value indicates that the wells tap a reservoir composed of waters older than 50 years. 


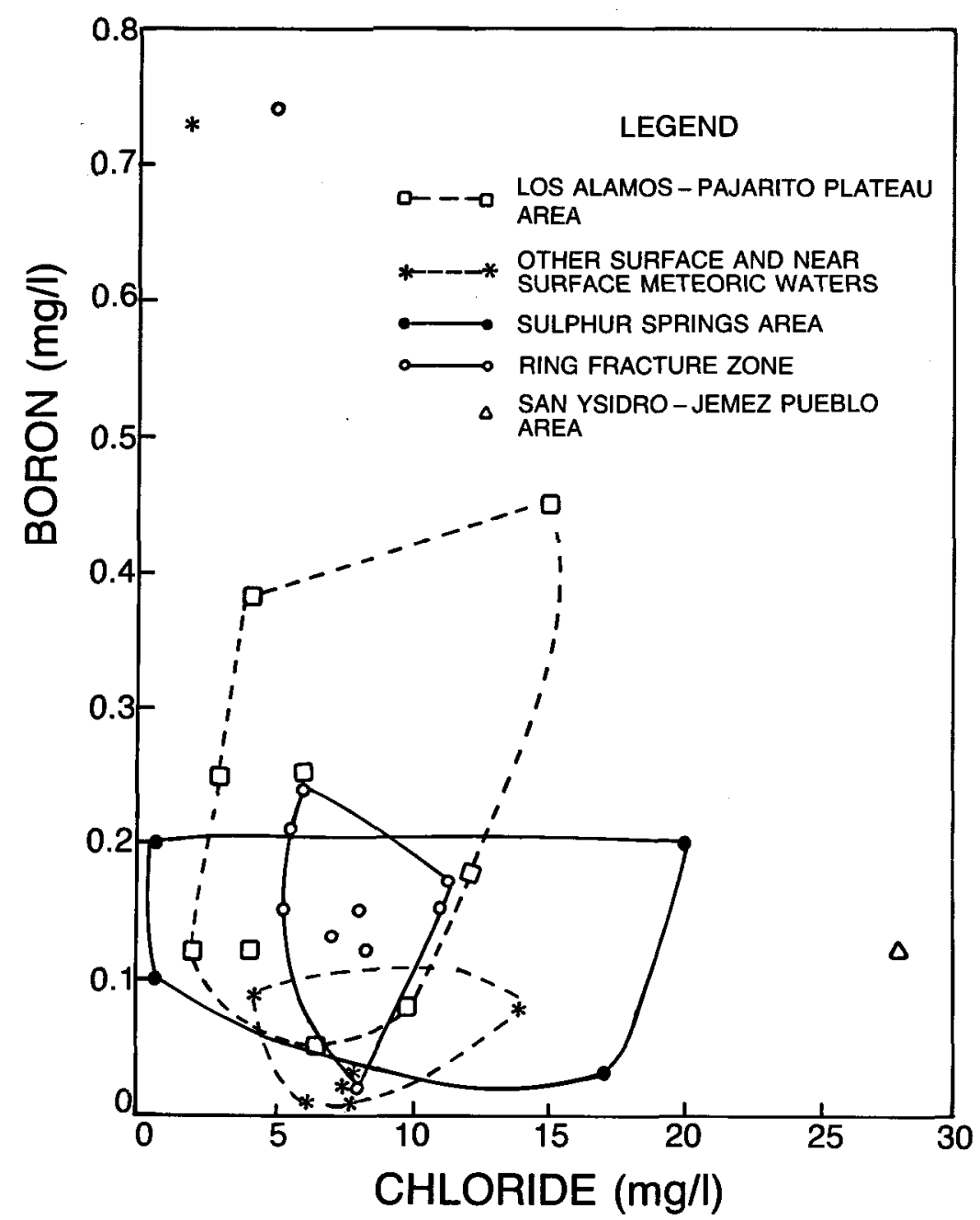

Fig. 10a.

Plot of $\mathrm{B}$ vs. $\mathrm{Cl}$ for waters with $\mathrm{Cl}$ concentrations 1 ess than $50 \mathrm{mg} / \mathrm{l}$ in the southern Jemez Mountains region, New Mexico.

Plots of $\delta D$ and $\delta^{18} 0$ of the Baca waters fall away from the world meteoric water line (Fig. 13b). These data show a distinct oxygen shift indicating an ${ }^{18} 0$ enrichment caused by high-temperature rock/water interaction.

\section{E. Valles Caldera-Soda Dam and Jemez Springs Area}

A number of deep thermal and derivative waters emerge as hot and warm springs along the trace of the Jemez fault zone in Cañon de San Diego. Springs at Soda Dam (Fig. 4) and springs near the village of Jemez Springs (Fig. 5) are included in this area. The Jemez fault zone extends southwest and south from the caldera for about $20 \mathrm{~km}$. It was active before eruption of the Jemez Mountains volcanic field (Goff et al. 1981) and locally represents 


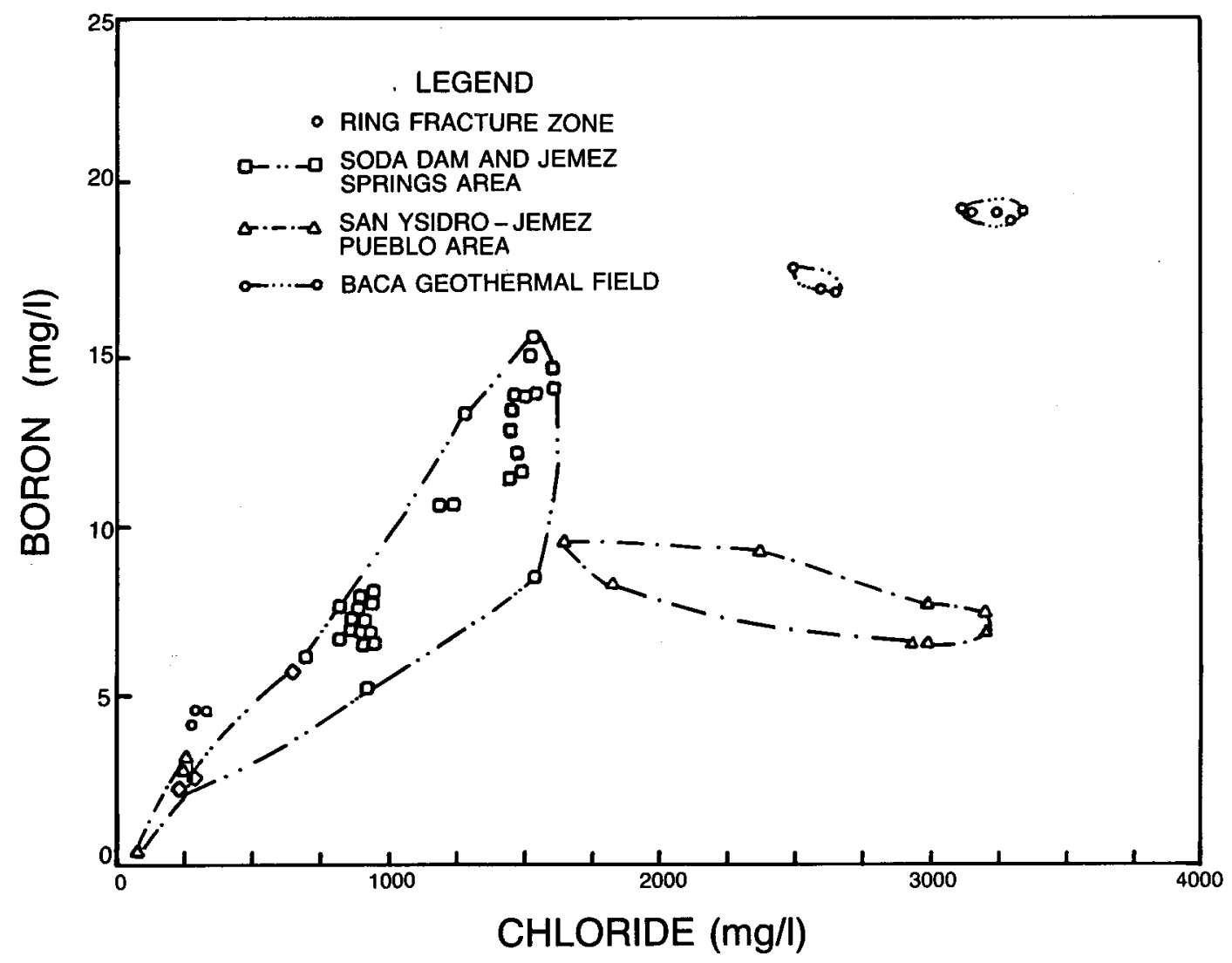

Fig. 10b.

Plot of $B$ vs. $C l$ for water with $\mathrm{Cl}$ concentrations greater than $50 \mathrm{mg} / \mathrm{l}$ in the southern Jemez Mountains region, New Mexico.

the western boundary of the Rio Grande rift. The hot springs occur at intersections of the Jemez River with the fault and fracture zones. Chemical compositions and origin of these mineralized springs have been discussed by Goff et a1. (1981), Trainer (1974), and in part by Goff and Grigsby (1982) and Goff and Sayer (1980). Soda Dam, Travertine Mound Spring, and some others are actively depositing carbonate travertine, and over $1 \mathrm{~km}^{2}$ of ancient travertine overlies the bluff above Soda Dam on the western side of the Jemez River.

The springs display fairly high conductivity and are neutral to slightly acid sodium-chloride to calcium-bicarbonate waters. The $\mathrm{Cl}$ and $\mathrm{Ca}$ concentrations show a slight inverse relationship suggesting a mixing of deep thermal water and surface meteoric water. They contain moderate $F$ and high Li and $B$ concentrations with a marked trend of increasing $B$ and $L i$ with increasing $\mathrm{Cl}$ (Figs. 9b, 10b, and 11b). 


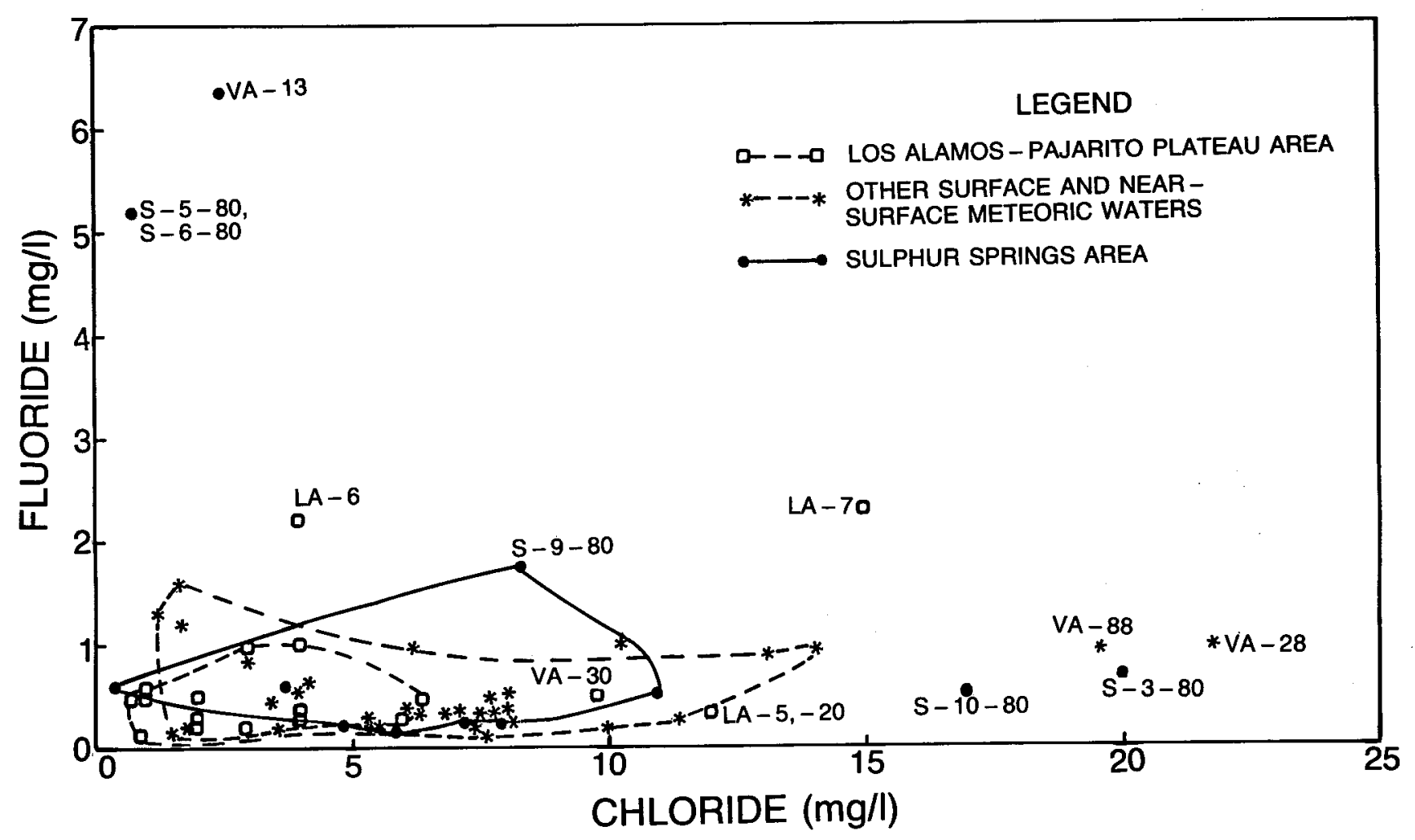

Fig. 11a.

Plot of $\mathrm{F}$ vs. $\mathrm{Cl}$ for thermal and nonthermal waters in the southern Jemez Mountains region, New Mexico. Sample numbers refer to waters that fall outside fields of generalized water types.

Strontium is a noteworthy minor element in these waters, ranging from 0.4 to $2 \mathrm{mg} / \mathrm{l}$ (Table B-III). There is an approximate trend of increasing $\mathrm{Sr}$ with increasing $\mathrm{Ca}$. The waters have probably derived most of their $\mathrm{Ca}$ and $\mathrm{HCO}_{3}$ by flowing through fractured Paleozoic carbonate rocks, which are probably the main source of $\mathrm{Sr}$. Arsenic is also enriched in these waters.

The $D$ and ${ }^{18} 0$ isotopes fall away from the meteoric line of Craig (1961), showing a distinct trend of ${ }^{18} 0$ enrichment (Fig. 13b). Water from Travertine Mound Spring and from the well near the main Jemez Spring is isotopically most similar to meteoric water, and the main Jemez and Soda Dam Springs show the most relative enrichment in 18 .

The tritium content of the springs is generaliy less than $7 \mathrm{~T} . \mathrm{U}$. and of ten less than 2 T.U., which implies a relatively old source water for the springs. The exception is at Buddhist Spring, which has a tritium content of 10 T.U. This higher tritium value is clearly the result of thermal water mixing with 


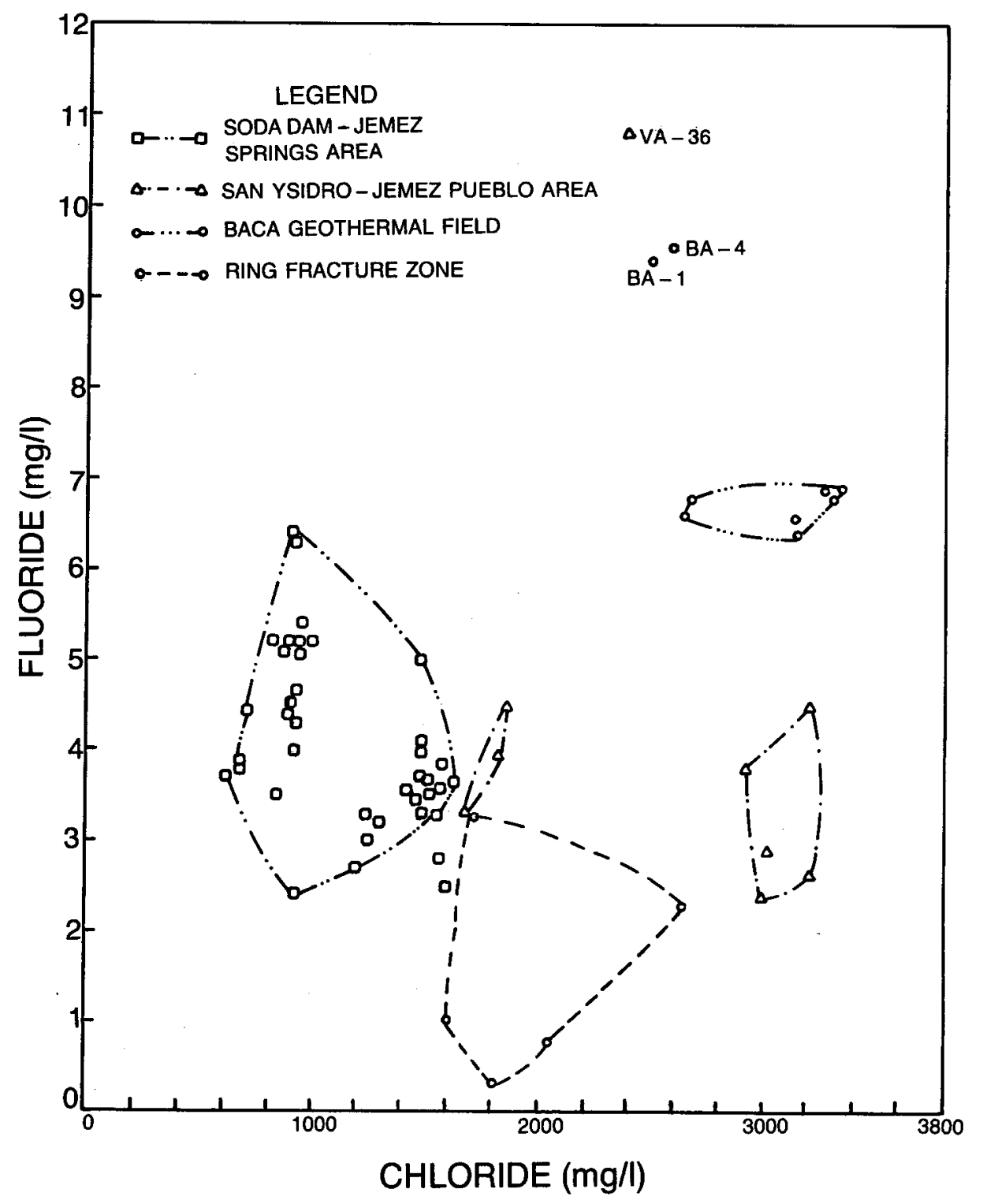

Fig. 11b.

Plot of $F$ vs. $\mathrm{Cl}$ for high-chloride thermal waters in the southern Jemez Mountains region, New Mexico. Sample numbers refer to waters that fall outside fields of generalized water types.

younger near-surface and surface waters, and water from the nearby Jemez River is a likely contributor to the high tritium values of Buddhist Spring.

These thermal waters are probably derivatives of the deep geothermal fluids beneath Valles Caldera, which are almost pure $\mathrm{NaCl}$ waters (Trainer 1974, Goff et a1. 1981). Mixing of this hot $\mathrm{NaCl}$ water with calciumbicarbonate meteoric water has cooled it down and diluted it. This mixed water dissolves $\mathrm{CaCO}_{3}$ while flowing southwest through shattered Paleozoic 


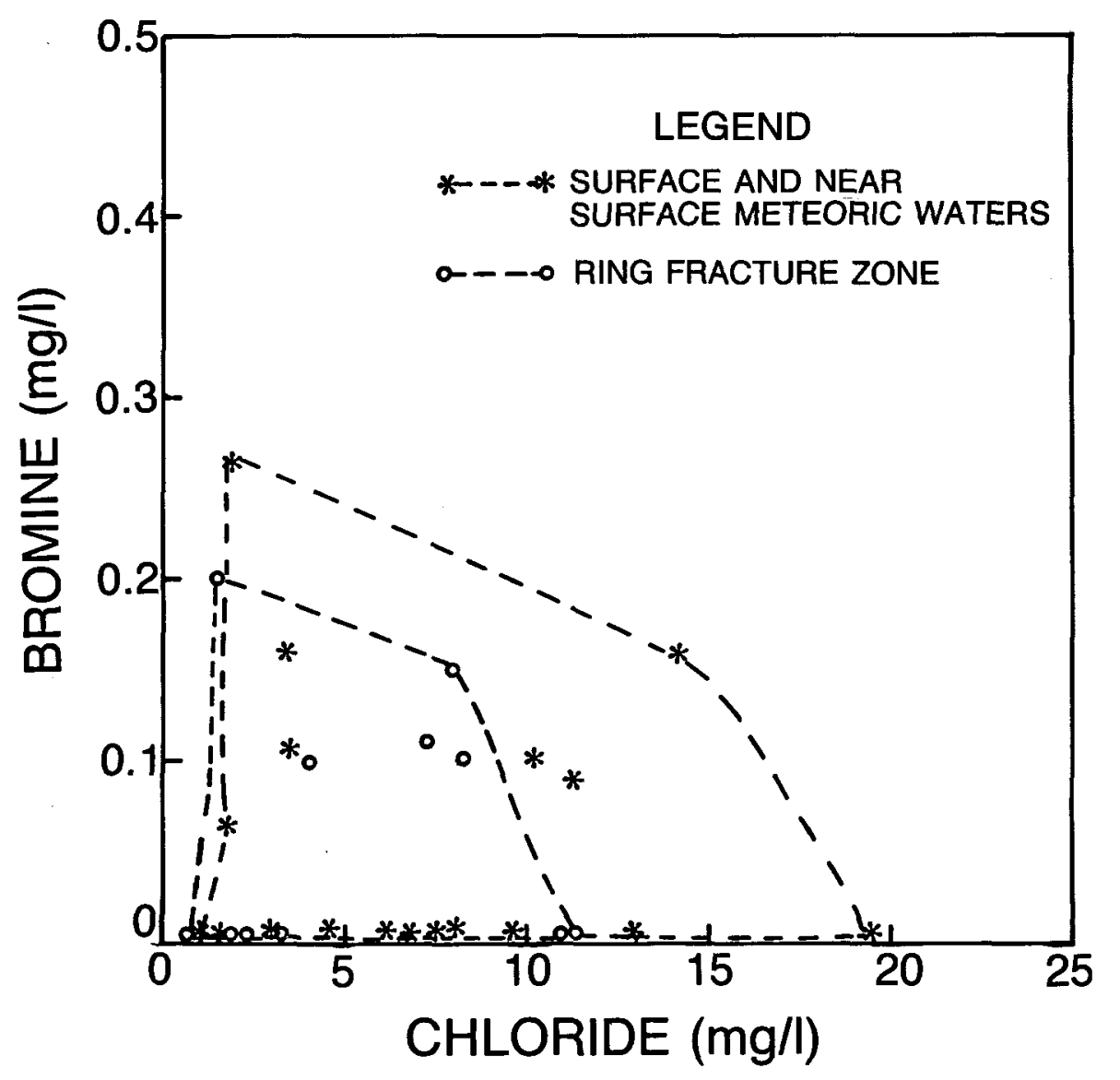

Fig $12 a$.

Plot of $\mathrm{Br}$ vs. $\mathrm{Cl}$ for waters with $\mathrm{Cl}$ concentrations 1 ess than $50 \mathrm{mg} / \mathrm{l}$ in the southern Jemez Mountains region, New Mexico. The only Br analyses available for low-Cl waters are from the ring fracture zone and surface and near-surface meteoric waters.

sediments along the Jemez fault zone, giving rise to the higher $\mathrm{Ca}$ and $\mathrm{HCO}_{3}$ contents observed in the springs. This mixing is supported by oxygen isotopes, since the deep geothermal water from Valles Caldera is much more enriched in ${ }^{18} 0$ relative to meteoric water (Goff and Grigsby 1982, Vuataz and Goff 1986).

F. San Ysidro-Jemez Pueblo Area

A number of warm springs and hot and cold water wells including mineral and nonmineral waters were sampled in the San Ysidro-Jemez Pueblo area. Many of the samples were collected for isotope analysis up to two years before they were collected for chemical analysis. The chemistry of the waters in this 


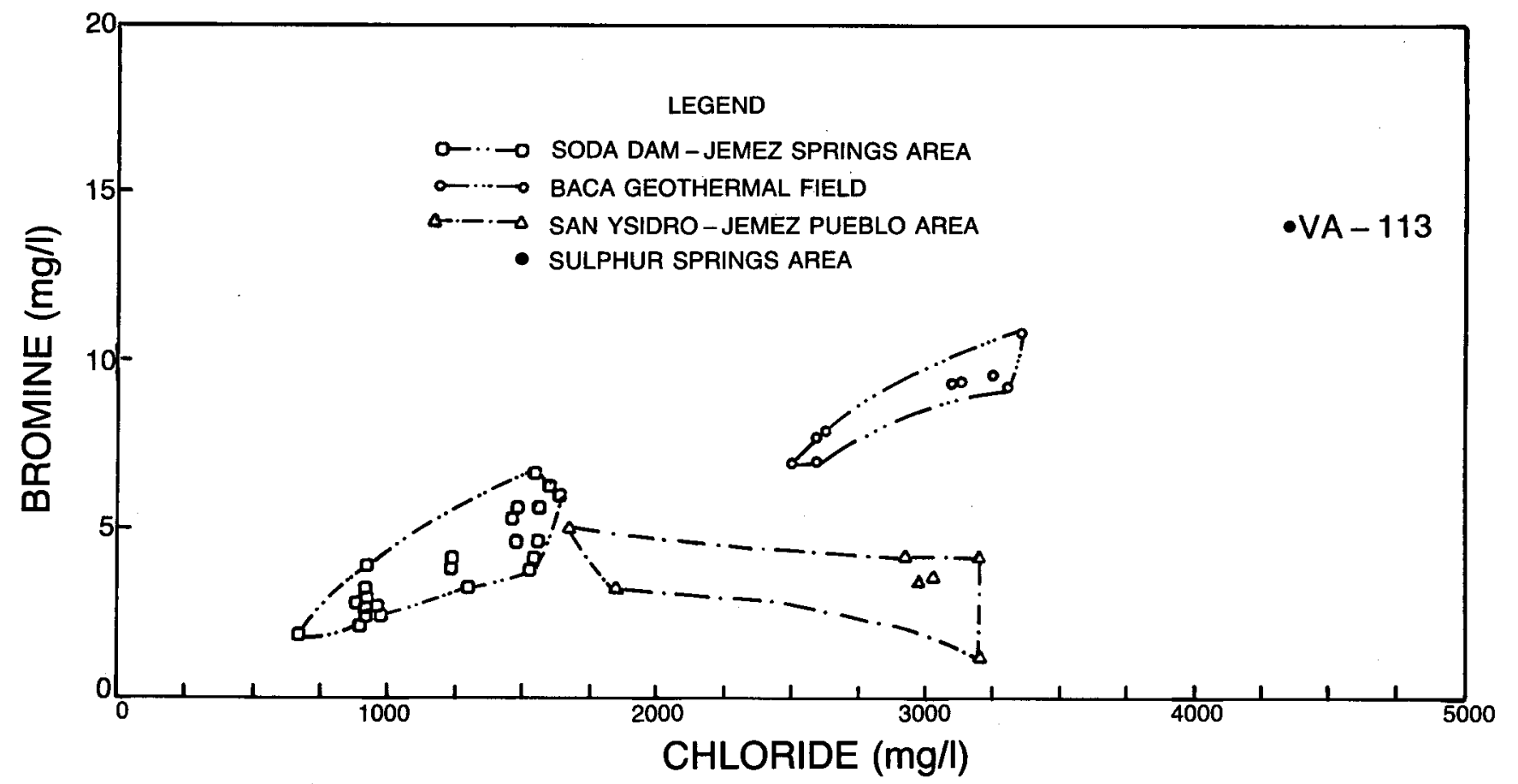

Fig. 12b.

Plot of $\mathrm{Br}$ vs. $\mathrm{Cl}$ for waters with $\mathrm{Cl}$ concentrations greater than $50 \mathrm{mg} / \mathrm{l}$ in the southern Jemez Mountains region, New Mexico. Sample number refers to waters that fall outside fields of generalized water types. Sample VA-116, from the GRI well, plots out of the field of view.

area has been disccussed by Goff et a1. (1981), Goff and Sayer (1980), Mariner et a1. (1977), Trainer (1974, 1975), and Vuataz and Goff (1986).

The waters are low to moderate temperature, near neutral, concentrated (except for dilute surface water) sodium chloride to sodium sulfate. They conta in much more $\mathrm{SO}_{4}, \mathrm{Na}$, and $\mathrm{Cl}$ thàn do the thermal waters discharging along the Jemez fault zone (Table B-II), except for Owl Spring, which resembles surface meteoric calcium-bicarbonate water. The concentrated waters have very high $\mathrm{Cl}$ and moderate to high F, B, and $\mathrm{Li}$ contents (Figs, 8b, 9a, 10a, and 11b). Silica contents are much lower than are most other waters described in this report, al though concentrations of silica increase with temperature (Fig. 7a).

Trace element concentrations are low except for $\mathrm{Sr}$, which increases with Ca concentration (Table B-III). Strontium contents are much higher in this area than in waters from the Jemez fault zone, which have comparable Ca.

Most of the $\mathrm{SO}_{4}$ in these waters may be derived from massive gypsum deposits (Jurassic Todilto Formation) near San Ysidro. Most of the springs issue from pre-Jurassic sediments, so the $\mathrm{SO}_{4}$ is probably derived both from 


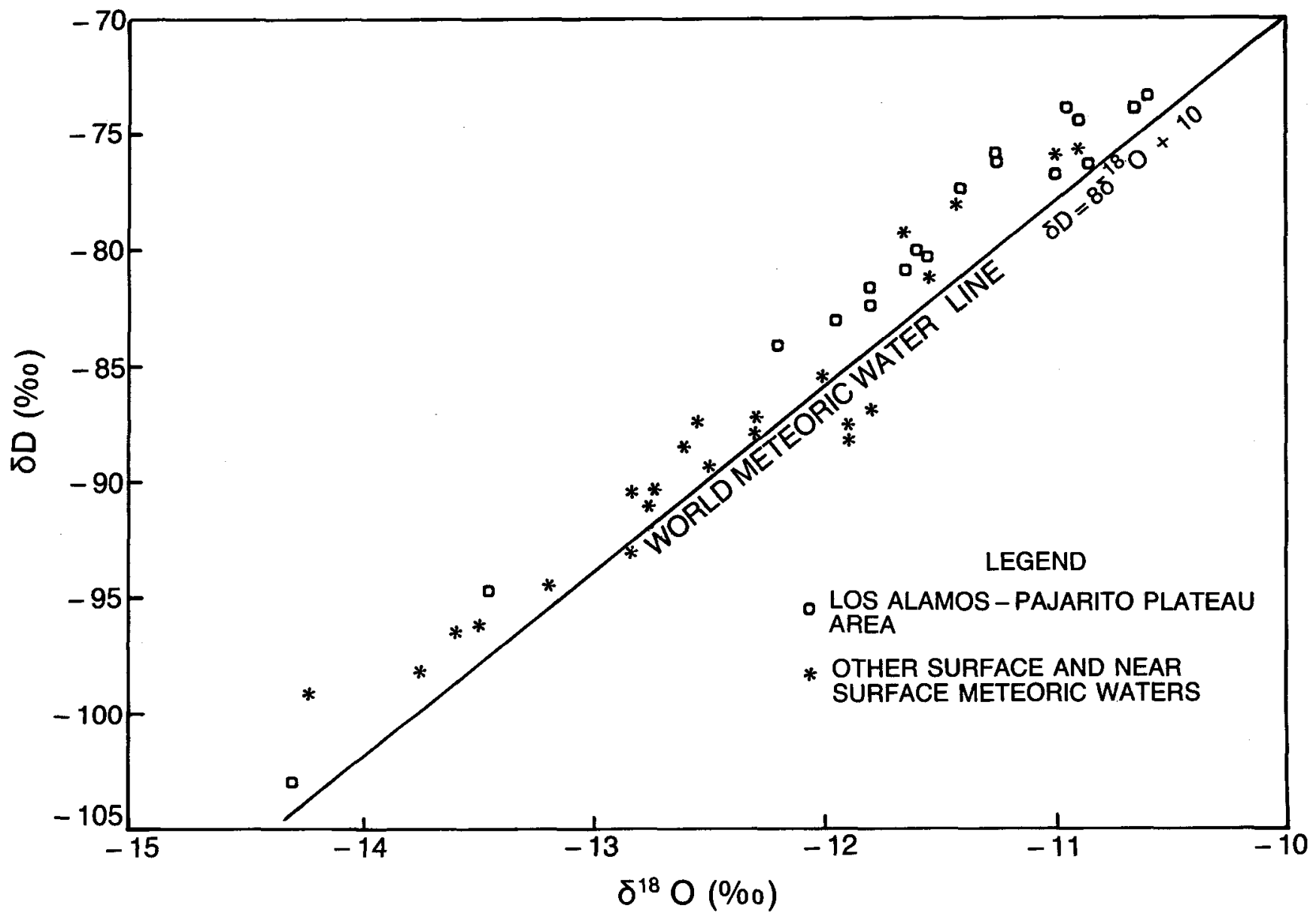

Fig. $13 a$.

Plot of $\delta D$ vs. $\delta^{18} 0$ for surface and near-surface meteoric waters in the Jemez Mountains and Los Alamos-Pajarito Plateau areas.

pore fluids in the rocks and from dowriward percolating groundwater. If we assume that $\mathrm{Ca}$ is also derived in part from gypsum, the higher $\mathrm{Sr} / \mathrm{Ca}$ in these waters compared with the Jemez and Soda Dam Springs may result from greater leaching of $\mathrm{Sr}$ from gypsum than from carbonate.

Several chemical features make these waters distinct from the waters derived from the deep geothermal system in the Valles Caldera (Goff et al. 1981). The ratios $\mathrm{Na} / \mathrm{Cl}$ and $\mathrm{B} / \mathrm{Cl}$ in the Jemez Springs waters are $1.3-1.9$ and 0.008-0.009, respectively, whereas the same ratios in the San Ysidro waters are 0.8-1.2 and 0.002-0.005, respectively. Li/Na ratios in waters from the Jemez Springs area are in the range 0.012-0.019 compared with 0.002-0.CJ5 for waters from the San Ysidro region. Finally, the isotopic enrichment of ${ }^{18} 0$ in San Ysidro waters follows a parallel but different trend (Fig. 13b) than do deep caldera waters. The tritium values obtained for San Ysidro Springs and 


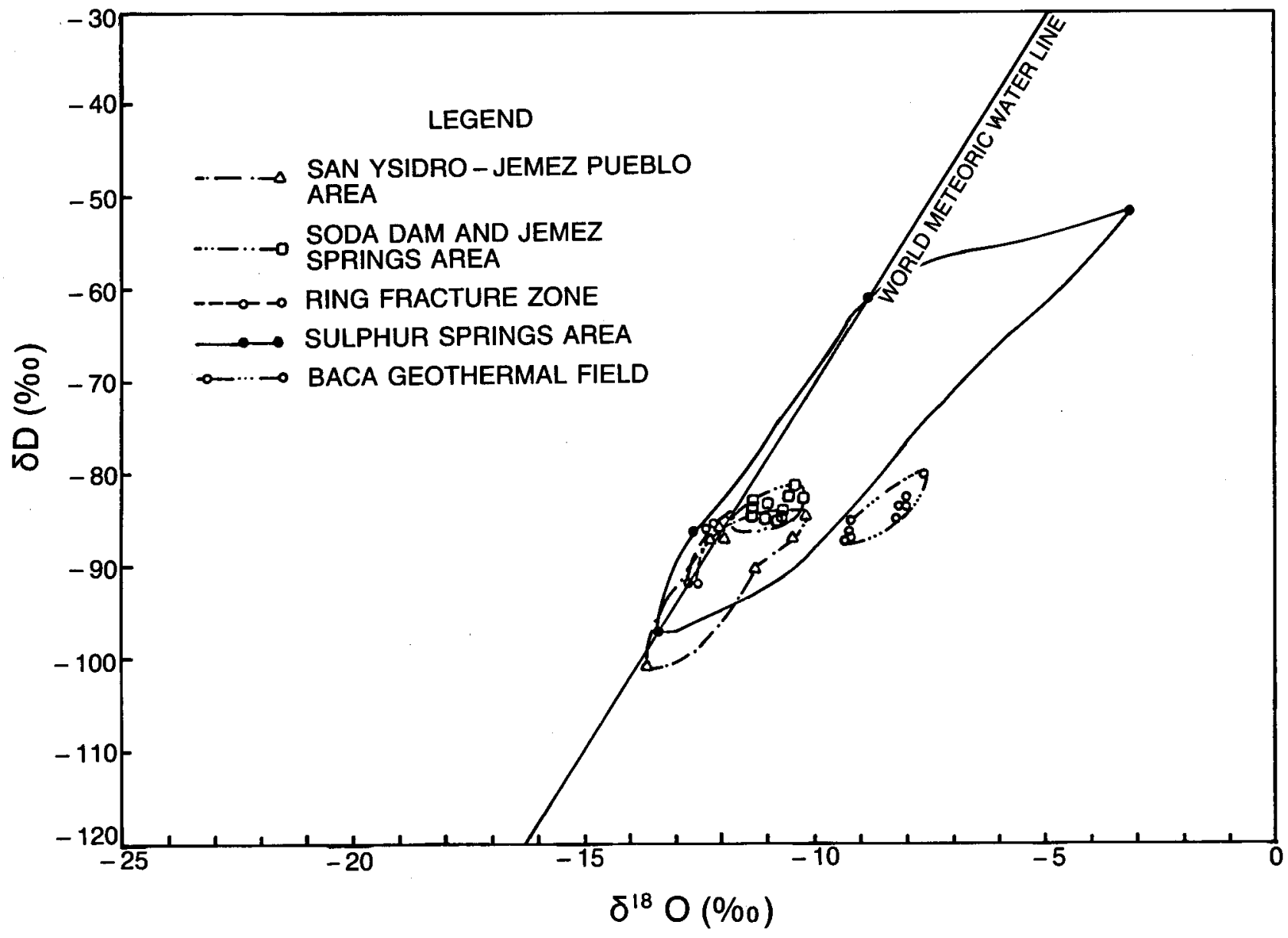

Fig. 13b.

Plot of $\delta D$ vs. $\delta^{18} 0$ for various types of water in the southern Jemez Mountains region, New Mexico.

the Zia hot well are $0.40 \mathrm{~T} . \mathrm{U}$. and $0.05 \mathrm{~T} . \mathrm{U} .$, respectively. These waters are therefore very old, much greater than 50 years old. All these data indicate that the San Ysidro waters originate from a separate low-temperature geothermal system near the Nacimiento fautt zone rather than from the one located beneath Valles caldera.

\section{SUMMARY}

The geologic, chemical, and isotopic data presented here are sufficient to characterize the different thermal and nonthermal waters of the valles Caldera-southern Jemez Mountains region. Further interpretations are beyond the scope of this report. However, the authors feel these data can be applied to the solution of other hydrologic and geochemical problems of the region. 


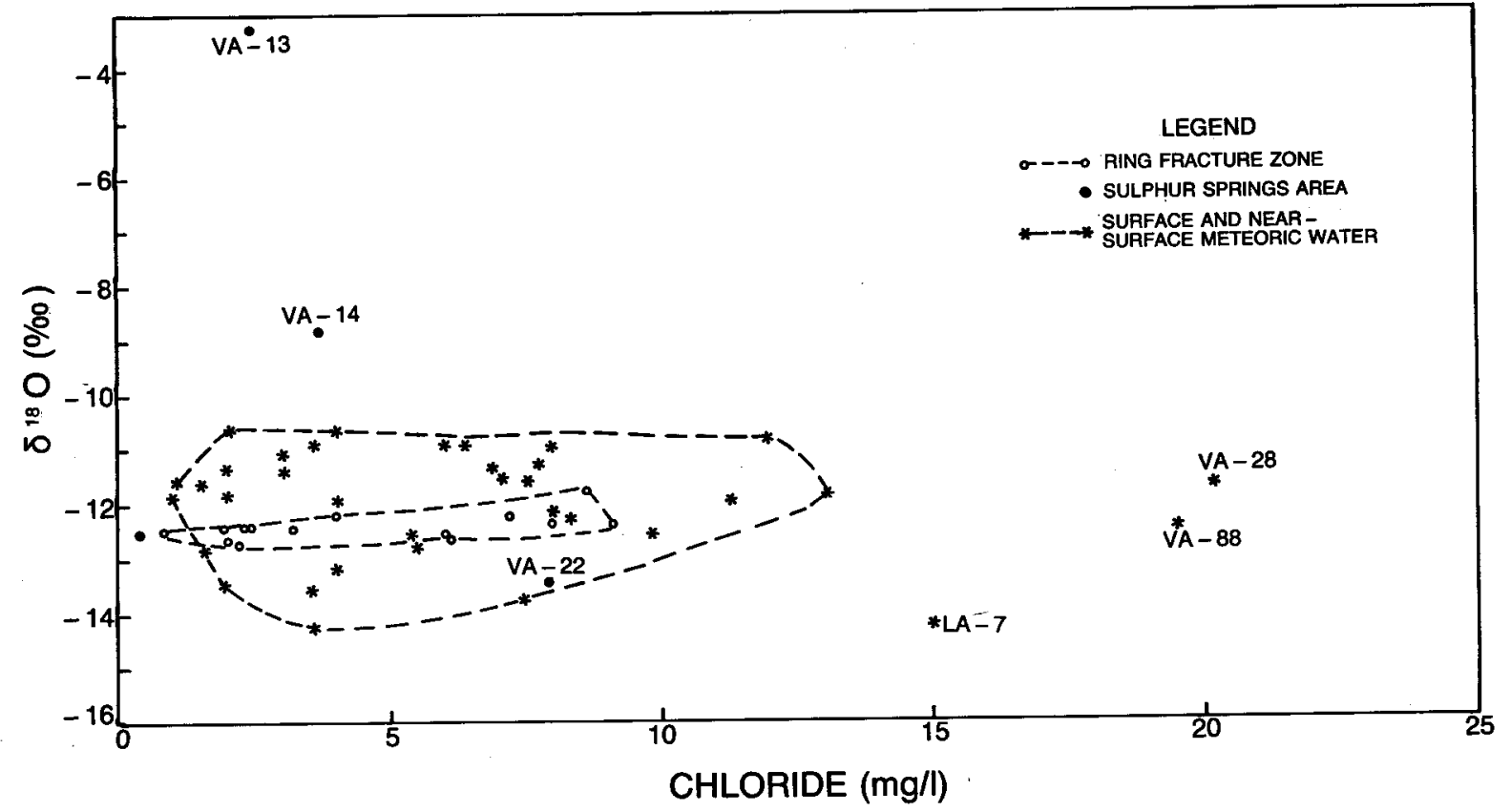

Fig. $14 a$.

Plot of $\delta^{18} 0$ vs. Cl for low-chloride waters in the southern Jemez Mountains region, New Mexico. Sample numbers refer to waters that fall outside fields of generalized water types.

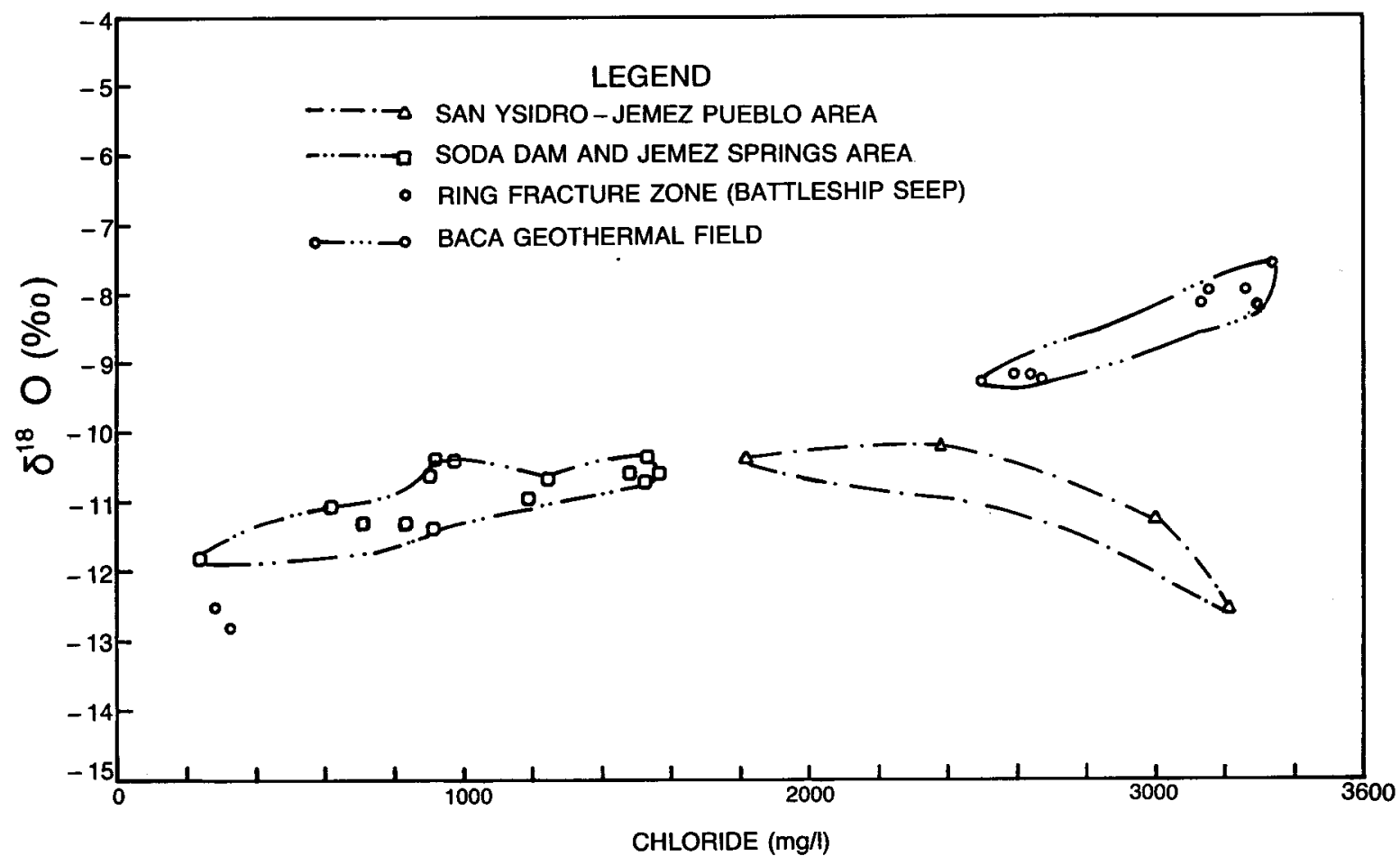

Fig. 14b.

Plot of $\delta^{18} 0$ vs. Cl for high-chloride thermal waters in the southern Jemez Mountains region, New Mexico. 
Perhaps, the most interesting problem would be to determine the age, geochemical evolution, and hydrologic balance of the high-temperature geothermal system within Valles Caldera.

\section{ACKNOWLEDGMENTS}

Many people at Los Alamos have contributed to the collection and analysis of waters listed in this report: Ron Aguilar, Kevin Ferdinand, Jamie Gardner, Chuck Grigsby, Kim Kariya, Tino Lucero, Dave Mann, Tamie McCormick, Suzanne Sayer, Bruce Stewart, and Rosemary Vidale. The previous work of Frank Trainer and Bill Purtymun is gratefully acknowledged. This work was supported by the US Department of Energy, Office of Basic Energy Sciences. 



\section{APPENDIX A}

UNUSUAL SPRINGS OF VALLES CALDERA AND SOUTHERN JEMEZ MOUNTAINS, NEW MEXICO

I. SULPHUR SPRINGS, VALLES CALDERA

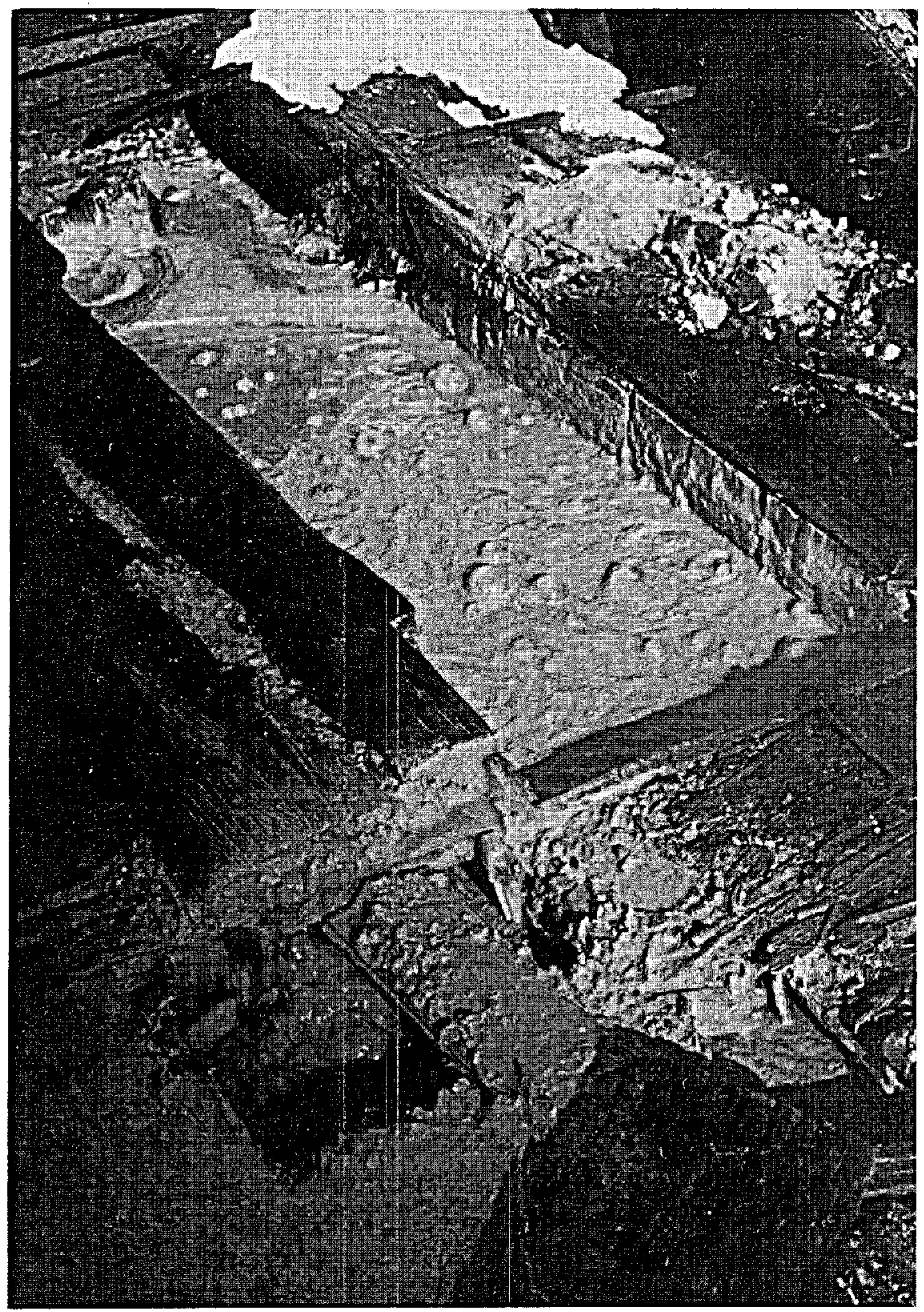

Fig. A-1.

Men's Bathhouse Mudpot in January 1979; $\mathrm{pH} \simeq 2.0$; temperature $\simeq 80^{\circ} \mathrm{C}$. The bathhouse, which is now collapsed, once held several concrete pools of muddy acid-sulfate water at various temperatures suitable for bathing. The mudpot is on a small knoll from which several fumaroles, $93^{\circ} \mathrm{C}$, discharge sulfurous gases. 


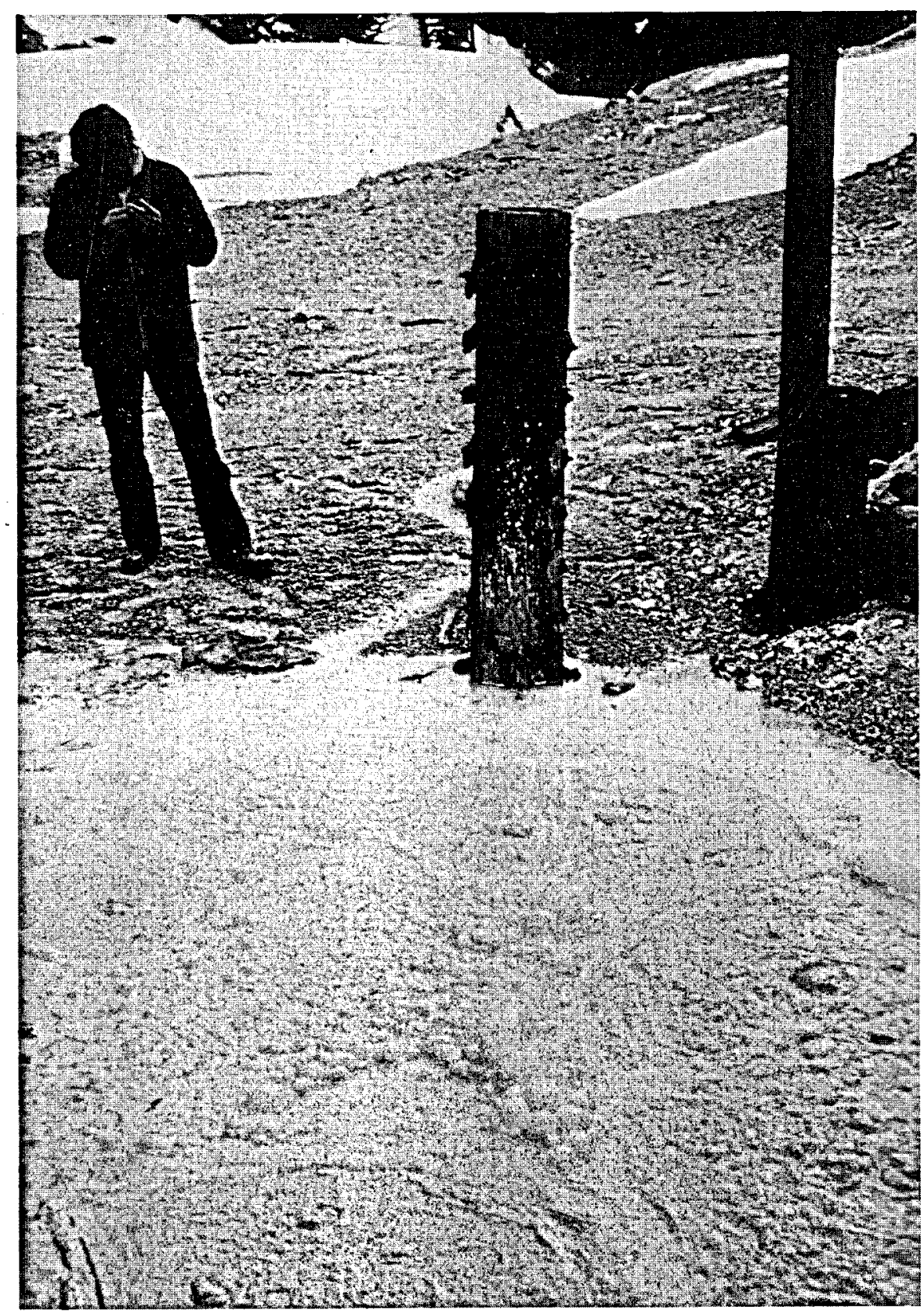

Fig. A-2.

Footbath Spring in March 1980; $\mathrm{pH} \cong 1.0$; temperature is highly variable. The pale yellow color of the suspended mud is due to colloidal sulfur. 


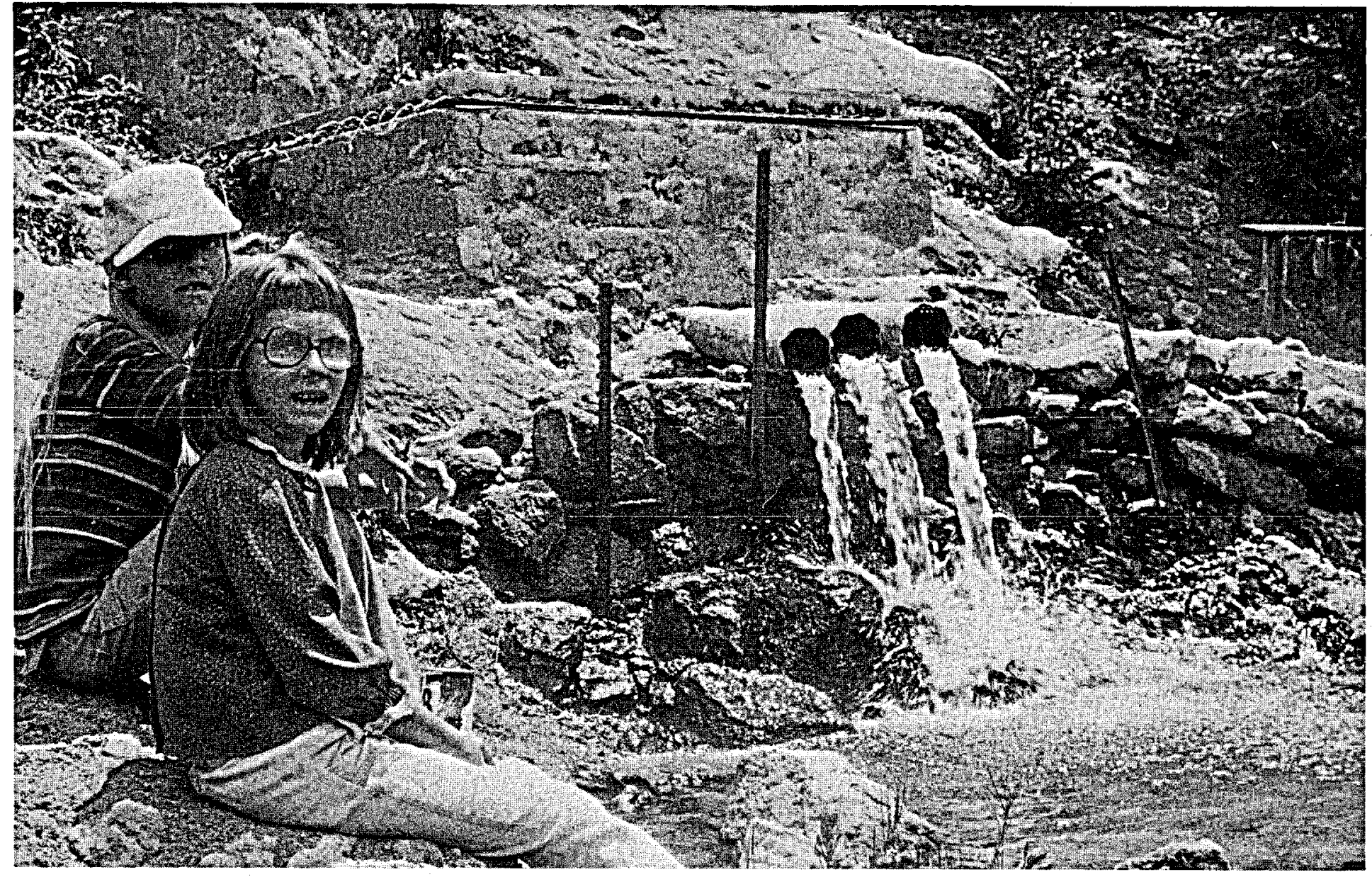

Fig. A-3.

San Antonio Hot Spring in July 1978; $\mathrm{pH} \simeq 7$; temperature $=42^{\circ} \mathrm{C}$. Spring discharges from concrete crib on $\omega$ fractured rhyolite about $60 \mathrm{~m}$ above San Antonio Creek, a favorite destination of cross-country skiers. 


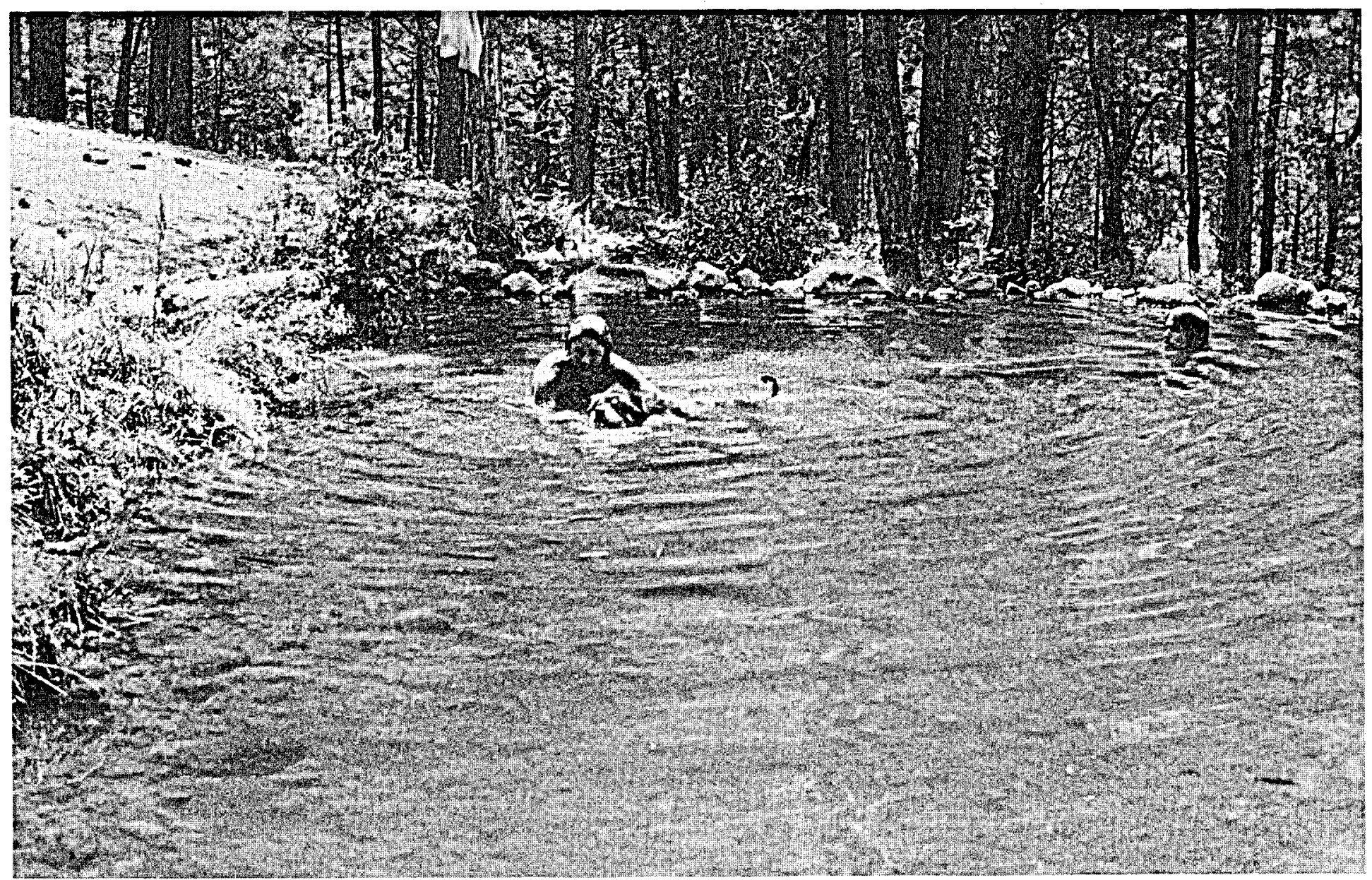

Fig. A-4

McCauley Spring in July 1978; $\mathrm{pH} \simeq 6.5$; temperature $=31^{\circ} \mathrm{C}$. Visitors have built a rock swimming pool about $30 \mathrm{~m}$ wide and $1 \mathrm{~m}$ deep adjacent to the spring orifice. They have also stocked it with tropical freshwater fish. 
III. JEMEZ FAULT ZONE, $10 \mathrm{~km}$ SOUTHWEST OF VALLES CALDERA

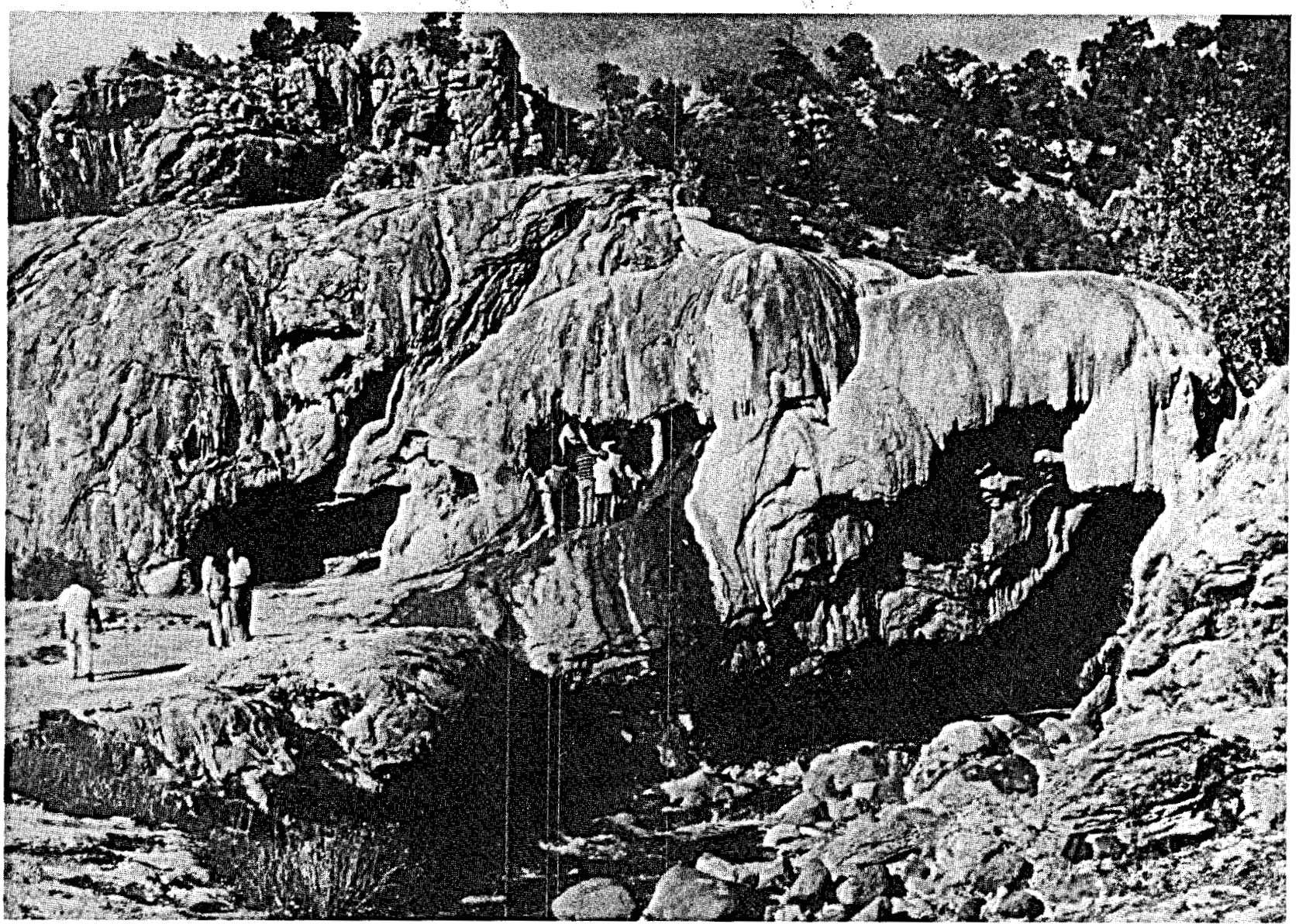

Fig. A-5.

Soda Dam looking north, April 1980. The dam is built of carbonate travertine deposited by bicarbonated hot springs, but construction of the present State Highway 4 (just to the left of the photograph) ruined the spring system. The existing dam is now disintegrating. The Jemez River has undercut the dam on the right of the photograph. Sightseers are standing at the entrance of Grotto Spring. 


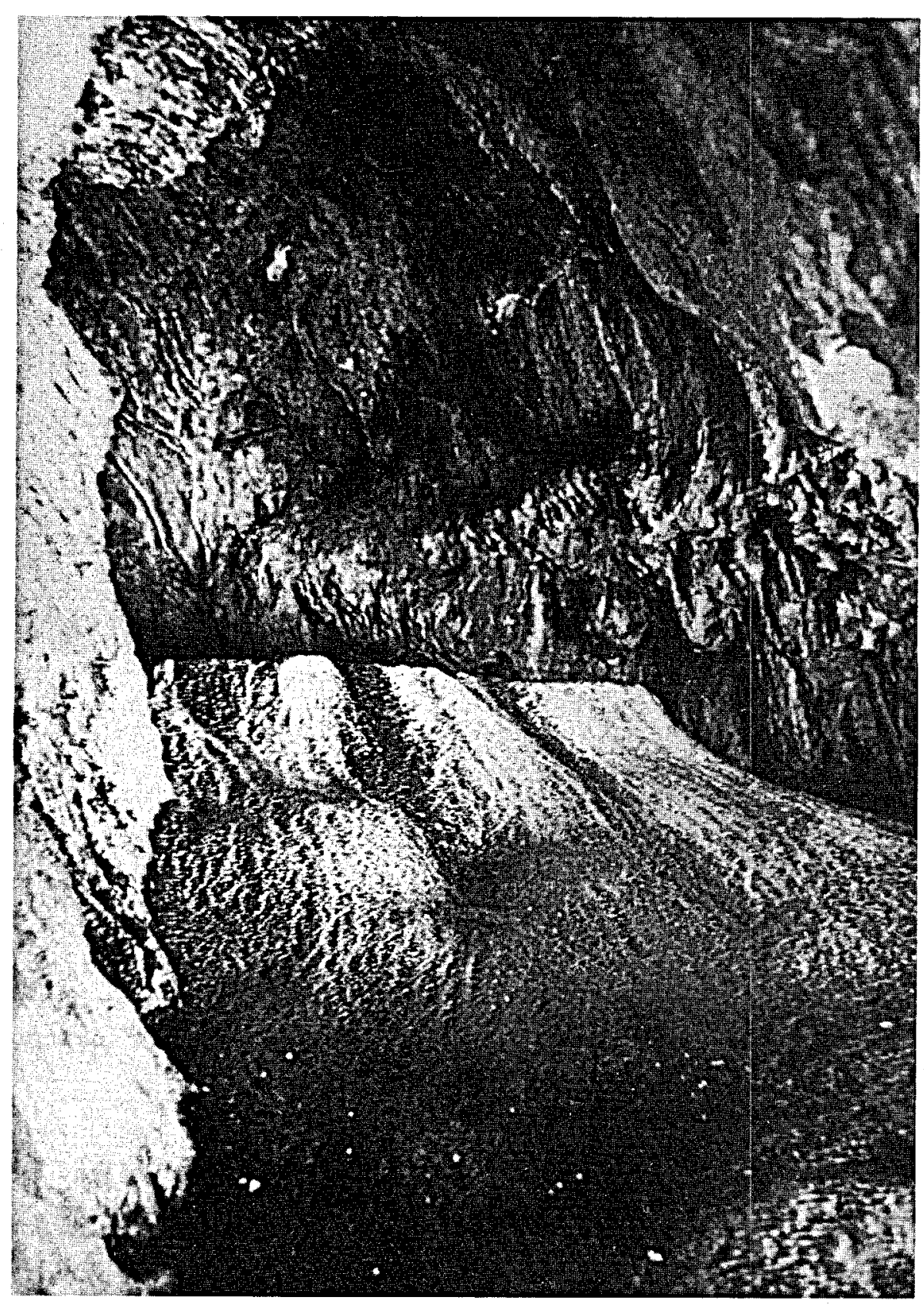

Fig. A-6.

Grotto Spring in october $1980 ; \mathrm{pH} \simeq 7$; temperature $=38^{\circ} \mathrm{C}$. This spring discharges from the cave in Soda Dam shown on the previous page. Although cooler in temperature, Grotto Spring has chemistry identical to that of Soda Dam Spring. 


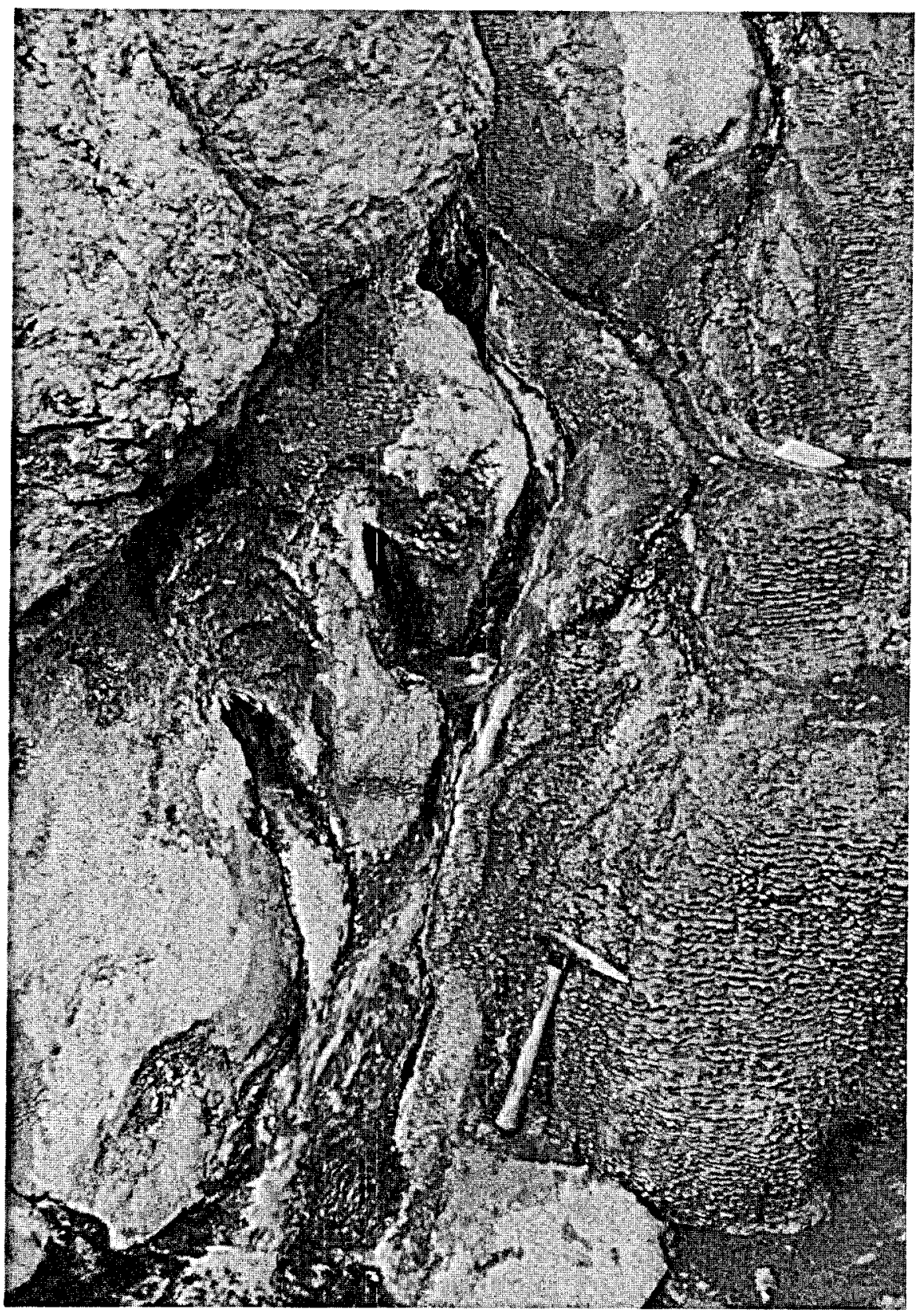

Fig. A-7.

The main spring at Soda Dam in October $1980 ; \mathrm{pH} \approx 6.3$; temperature $=47^{\circ} \mathrm{C}$. Spring issues from a shear zone separating granite from vertically faulted sandstone along the edge of State Highway 4. 


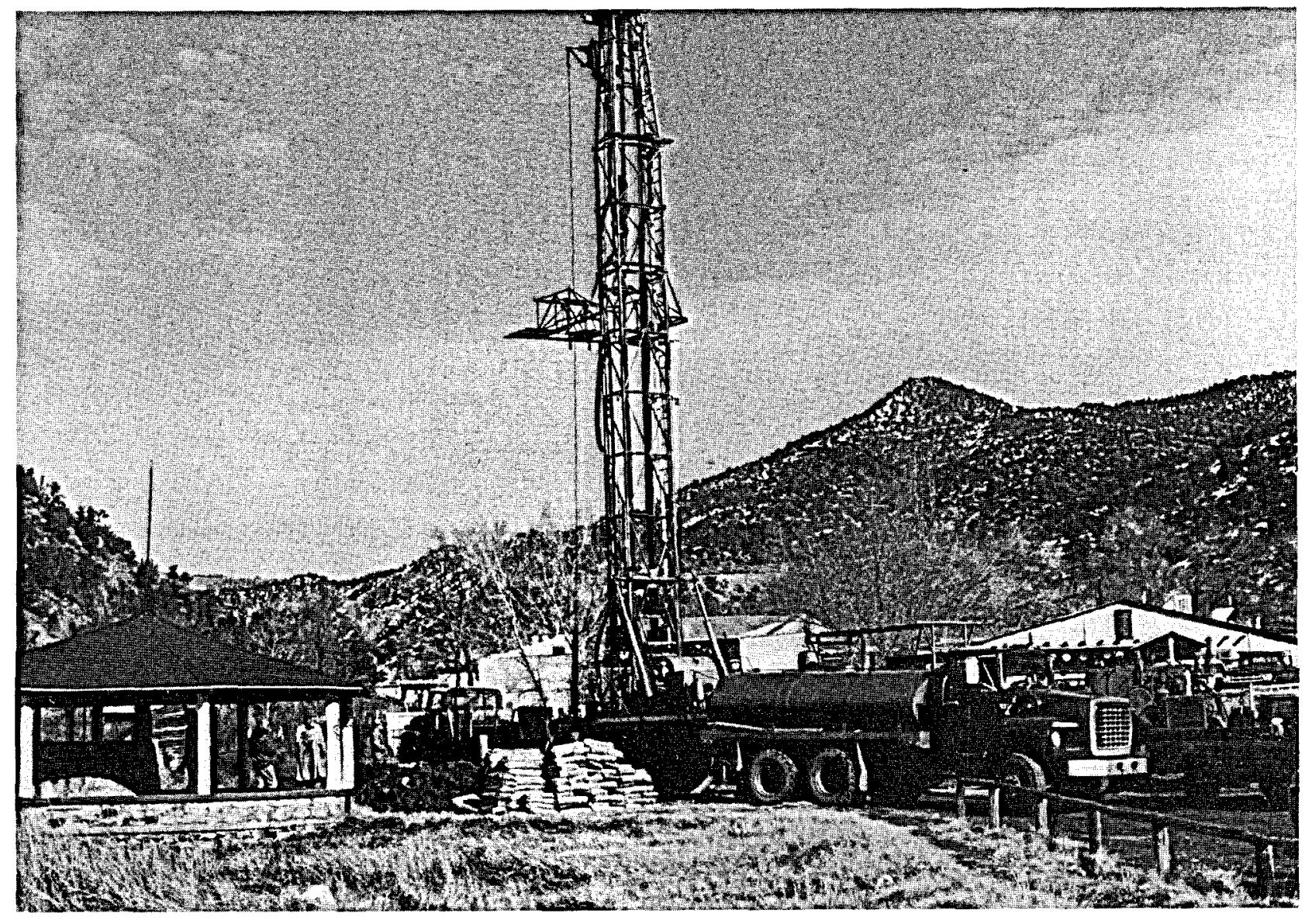

Fig. A-8.

Main Jemez Springs during drilling of Jemez Springs Geothermal Well, January 1979. The gazebo covers the main spring, really an old shallow well, that supplies water to the bathhouse (behind photographer). The geothermal well struck hottest water, $72^{\circ} \mathrm{C}$, at $24-\mathrm{m}$ depth, al though total depth attained was $255 \mathrm{~m}$. The hot water from this well is currently being used for space heating. 


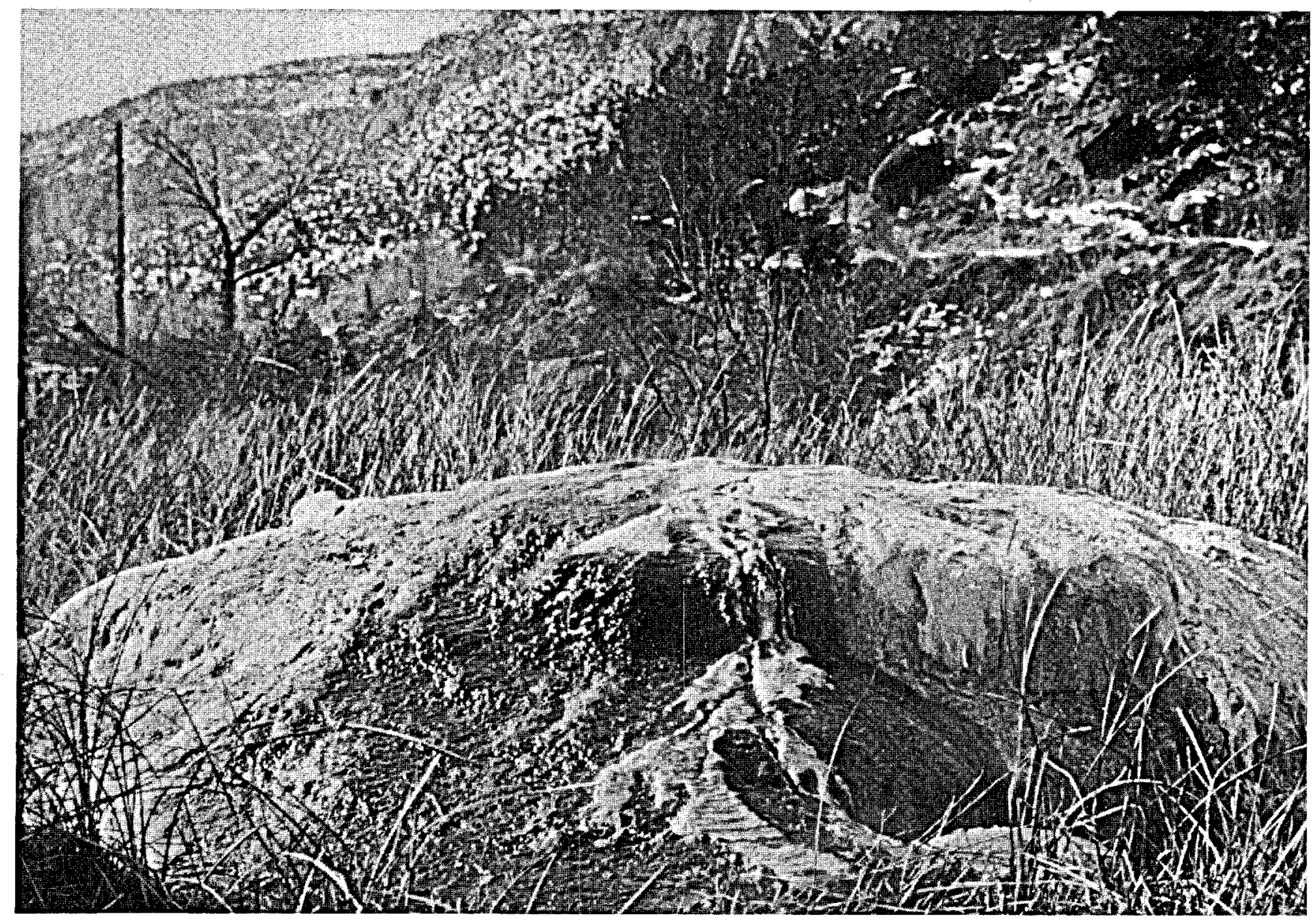

Fig. $A-9$.

Travertine Mound Spring in January 1979; $\mathrm{pH} \simeq 6.4$; temperature $=72^{\circ} \mathrm{C}$. This spring, which discharges between the bathhouse and the Jemez River, contains an extremely rare species of algae. 


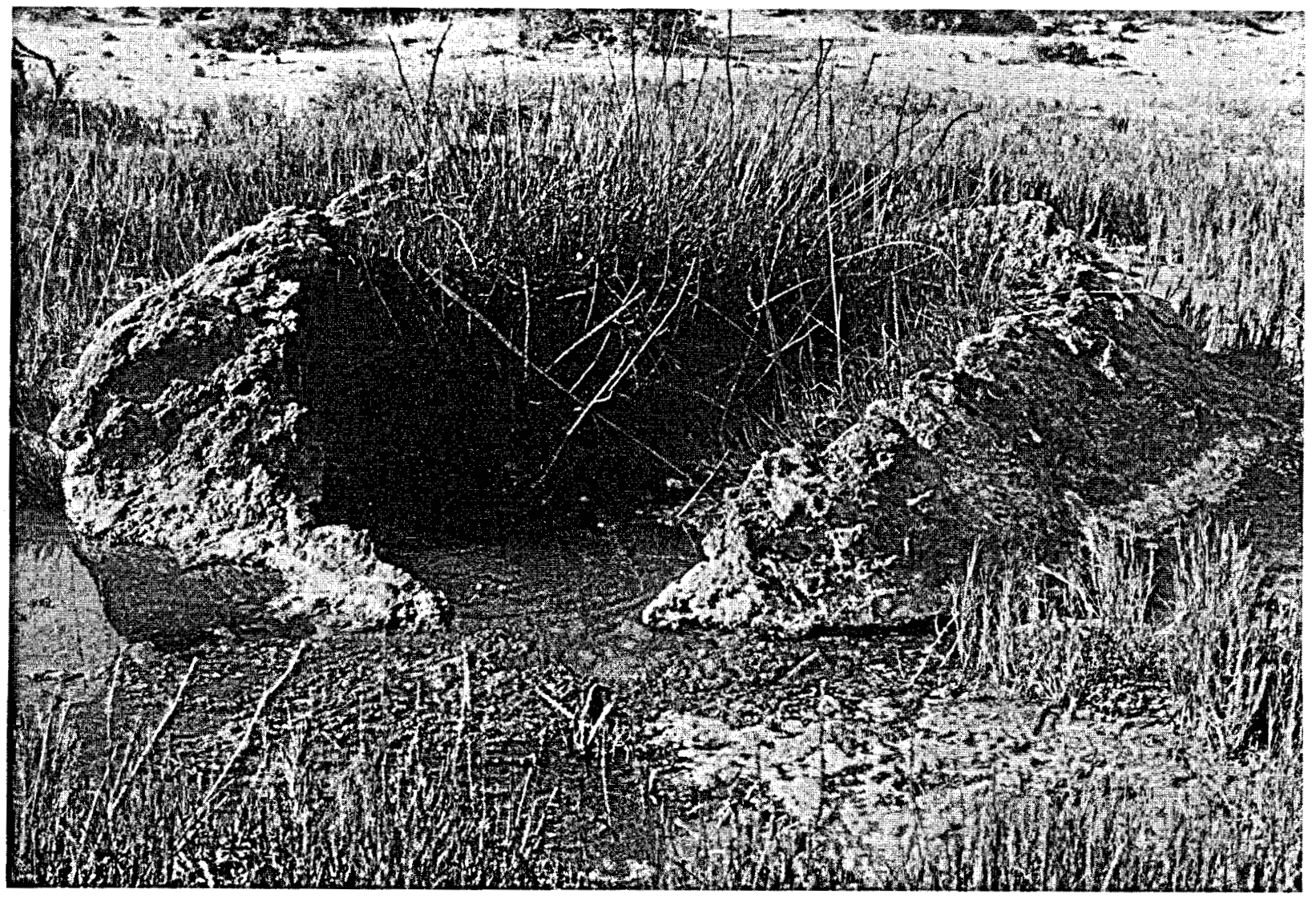

Fig. $A-10$.

San Ysidro Warm Spring in August 1979; $\mathrm{pH} \simeq 6.5$; temperature $\simeq 25^{\circ} \mathrm{C}$. The spring discharges about $20 \mathrm{~m}$ north of State Highway 44 and resembles four or five others within $200 \mathrm{~m}$. Water' is rich in dissolved sulfate that is probably leached from gypsum-bearing rocks nearby. 


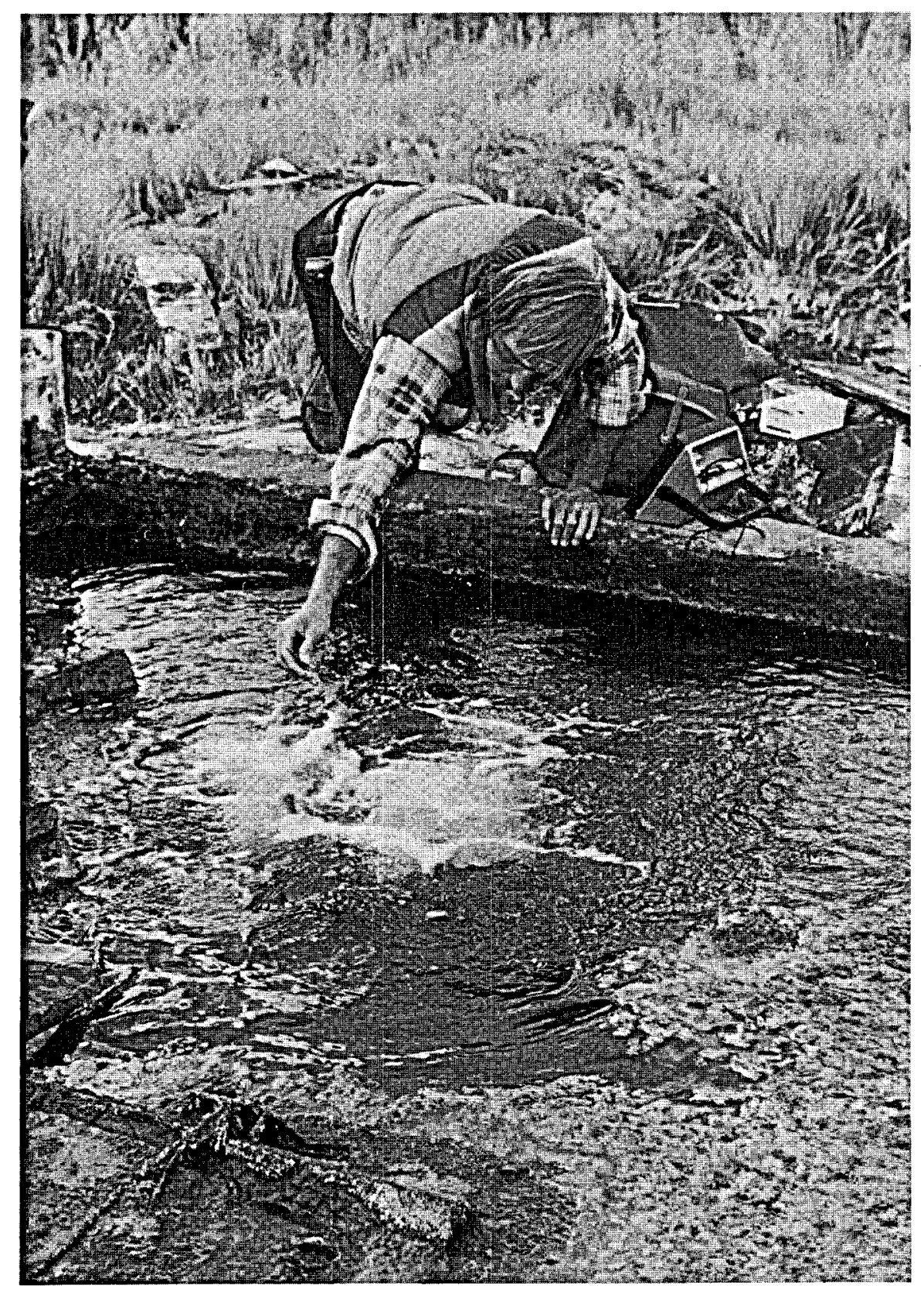

Fig. A-11.

Zia Hot Well (Kaseman \#2 0il Test Well) in Apri1 1980; $\mathrm{pH} \approx 6.5$; temperature $=$ $54^{\circ} \mathrm{C}$. The well was drilled in 1926 as an oil test well but struck hot water with an artesian flow of approximately $300 \mathrm{l} / \mathrm{min}$. A notel built adjacent to the well burned down in the 1950s. Now unused, this well could supply substantial geothermal space heat or heat for greenhouses. 


\section{APPENDIX $B$}

GEOCHEMICAL DATA OF THERMAL AND NONTHERMAL WATERS OF THE VALLES CALDERA AND THE SOUTHERN JEMEZ MOUNTAINS, NEW MEXICO 
LOCATION, FIELD DATA, AND REFERENCES FOR WATERS IN THE JEMEZ MOUNTAINS REGION, NEW MEXICO

\begin{tabular}{|c|c|c|c|c|c|c|c|c|c|c|c|c|}
\hline $\begin{array}{l}\text { Map } \\
\text { No. }\end{array}$ & Name & $\begin{array}{l}\text { Field } \\
\text { No. }\end{array}$ & Date & Location & $\begin{array}{l}\text { Water } \\
\text { Type }\end{array}$ & $\left({ }^{\circ} \mathrm{C}\right)$ & $\underset{\text { pH }}{\text { Field }}$ & $\begin{array}{l}\text { Field } \\
\text { Eh(mv) }\end{array}$ & $\begin{array}{l}\text { Conduc. } \\
\text { (umhos/cm) }\end{array}$ & $\begin{array}{c}\text { Flow } \\
\text { Rate } \\
(\ell / \mathrm{min})\end{array}$ & Comments & Reference $^{b}$ \\
\hline \multicolumn{13}{|c|}{ Surface and Near-Surface Meteoric Waters } \\
\hline 1 & Gallery Spring & LA-1 & $8 / 78$ & $\begin{array}{l}\text { Lat } 35^{\circ} 50^{\prime} 38^{\prime \prime} \\
\text { Long } 106^{\circ} 22^{\prime} 17^{\prime \prime}\end{array}$ & $m$ & 11 & 5.6 & -- & -- & 160 & $\begin{array}{l}\text { Spring issues from concrete } \\
\text { gallery built in fractured } \\
\text { Bandelier Tuff; Frijoles } \\
\text { Quadrangle }\end{array}$ & $\begin{array}{l}\text { G-S; G et } \\
\text { al; ; P79; } \\
\text { T78 (R5) }\end{array}$ \\
\hline 2 & $T-3$ Well & LA-2 & $8 / 78$ & $\begin{array}{l}\text { Lat } 35^{\circ} 52^{\prime} 23^{\prime \prime} \\
\text { Long } 106^{\circ} 15^{\prime} 33^{\prime \prime}\end{array}$ & $m$ & 13 & 6.5 & -- & -- & $0-12$ & $\begin{array}{l}\text { Observation well w50 m deep, } \\
\text { iron casing; in alluvium and } \\
\text { Puye (?) Fm.; Frijoles Quadrangle }\end{array}$ & G-S \\
\hline 3 & T-2 Well & LA-3 & $8 / 78$ & $\begin{array}{l}\text { Lat } 35^{\circ} 53^{\prime} 06^{\prime \prime} \\
\text { Long } 106^{\circ} 16^{\prime} 12^{\prime \prime}\end{array}$ & m & 11 & 5.7 & -- & -- & $0-8$ & $\begin{array}{l}\text { Observation well } r 50 \mathrm{~m} \text { deep, } \\
\text { iron casing; in alluvium and } \\
\text { Puye (?) Fm.; Guaje Quadrangle }\end{array}$ & G-S \\
\hline 4 & Sacred Spring & LA-4 & $8 / 78$ & $\begin{array}{l}\text { Lat } 35^{\circ} 53^{\prime} 35^{\prime \prime} \\
\text { Long } 106^{\circ} 08^{\prime} 59^{\prime \prime}\end{array}$ & $m$ & 14 & 5.7 & -- & -- & Seep & $\begin{array}{l}\text { Seep discharges from Santa Fe } \\
\text { Fm.; Puye Quadrangle }\end{array}$ & $\begin{array}{l}\text { Get al; } \\
\text { T78 (T6) }\end{array}$ \\
\hline 5 & Basalt Spring & $L A-5$ & $8 / 78$ & $\begin{array}{l}\text { Lat } 35^{\circ} 52^{\prime} 03^{\prime \prime} \\
\text { Long } 106^{\circ} 11^{\prime} 44^{\prime \prime}\end{array}$ & $m$ & 15 & 5.8 & -- & -- & 4 & $\begin{array}{l}\text { Spring flows from contact of } \\
\text { Cerros del Rio basalt t and } \\
\text { Puye Fm.; White Rock Quadrangle }\end{array}$ & $\begin{array}{l}\text { G-S; } \\
\text { G et al. }\end{array}$ \\
\hline 6 & L-6 Well & LA-6 & $9 / 78$ & $\begin{array}{l}\text { Lat } 35^{\circ} 52^{\prime} 38^{\prime \prime} \\
\text { Long } 106^{\circ} 10^{\prime} 25^{\prime \prime}\end{array}$ & $\mathrm{m}$ & 27 & 6.8 & -- & -- & $0-2160$ & $\begin{array}{l}\text { Water-supply well drilled } 625 \mathrm{~m} \\
\text { into Santa Fe Fm.; steel casing; } \\
\text { Puye Quadrangle }\end{array}$ & $\begin{array}{l}\text { G-S; Gr; } \\
\text { P77; P79 }\end{array}$ \\
\hline 7 & $L-1 B$ Well & $\mathrm{LA}-7$ & $9 / 78$ & $\begin{array}{l}\text { Lat } 35^{\circ} 53^{\prime} 00^{\prime \prime} \\
\text { Long } 106^{\circ} 09^{\prime} 22^{\prime \prime}\end{array}$ & $m$ & 30 & 7.2 & -- & -- & $0-2180$ & $\begin{array}{l}\text { Water-supply well drilled } 694 \mathrm{~m} \\
\text { into Santa Fe Fm.; steel casing; } \\
\text { Puye Quadrangle }\end{array}$ & $\begin{array}{l}\text { G-S; P77; } \\
\text { P79; P-C }\end{array}$ \\
\hline 8 & $L-5$ We 11 & LA-8 & $9 / 78$ & $\begin{array}{l}\text { Lat } 35^{\circ} 53^{\prime} 18^{\prime \prime} \\
\text { Long } 106^{\circ} 11^{\prime} 01^{\prime \prime}\end{array}$ & $m$ & 26.5 & 6.5 & -- & -- & $0-1880$ & $\begin{array}{l}\text { Water-supply well drilled to } \\
623 \mathrm{~m} \text { into Santa Fe Fm.; steel } \\
\text { casing; White Rock Quadrangle }\end{array}$ & $\begin{array}{l}\text { G-S; Gr; } \\
\text { P77; P79; } \\
\text { P-C }\end{array}$ \\
\hline 9 & L-4 Well & LA-9 & $9 / 78$ & $\begin{array}{l}\text { Lat } 35^{\circ} 52^{\prime} 06^{\prime \prime} \\
\text { Long } 106^{\circ} 11^{\prime} 30^{\prime \prime}\end{array}$ & $m$ & 28 & 6.5 & -- & -- & $0-1560$ & $\begin{array}{l}\text { Water-supply well drilled to } \\
622 \mathrm{~m} \text { into Santa Fe Fm.; steel } \\
\text { casing; White Rock Quadrangle }\end{array}$ & $\begin{array}{l}\text { G-S; Gr; } \\
\text { P77; P79 }\end{array}$ \\
\hline 10 & PM-2 Hell & $L A-10$ & $9 / 78$ & $\begin{array}{l}\text { Lat } 35^{\circ} 50^{\prime} 21^{\prime \prime} \\
\text { Long } 106^{\circ} 15^{\prime} 53^{\prime \prime}\end{array}$ & $m$ & 23.5 & 6.5 & -- & -- & $0-5520$ & $\begin{array}{l}\text { Well drilled } 800 \mathrm{~m} \text { into santa Fe } \\
\text { Fm.; steel casing; Frijoles } \\
\text { Quadrangle }\end{array}$ & G-S; P79 \\
\hline 11 & PM-1 We11 & $L A-11$ & $9 / 78$ & $\begin{array}{l}\text { Lat } 35^{\circ} 51^{\prime} 32^{\prime \prime} \\
\text { Long } 106^{\circ} 13^{\prime} 27^{\prime \prime}\end{array}$ & m & 28 & 6.5 & -- & -- & $0-2320$ & $\begin{array}{l}\text { Well drilled } 470 \mathrm{~m} \text { into Santa Fe } \\
\text { Fm.; steel casing; White Rock } \\
\text { Quadrangle }\end{array}$ & G-S; P79 \\
\hline
\end{tabular}


TABLE B-I (cont)

\begin{tabular}{|c|c|c|c|c|c|c|c|c|c|c|c|c|}
\hline $\begin{array}{l}\text { Map } \\
\text { No. }\end{array}$ & Name & $\begin{array}{l}\text { Field } \\
\text { No. }\end{array}$ & Date & Location & $\begin{array}{l}\text { Water } \\
\text { Type }\end{array}$ & $\begin{array}{l}\text { Temp } \\
(* c)\end{array}$ & $\begin{array}{l}\text { field. } \\
\text { pH }\end{array}$ & $\begin{array}{c}\text { Field } \\
\text { Eh(mV) }\end{array}$ & $\begin{array}{l}\text { Conduc. } \\
\text { (mmos/cm) }\end{array}$ & $\begin{array}{l}\text { Flow } \\
\text { Rate } \\
\text { (l/min) }\end{array}$ & Comments & Reference ${ }^{b}$ \\
\hline 12 & G-6 Well & LA-12 & $9 / 78$ & $\begin{array}{l}\text { Lat } 35^{\circ} 54^{\prime} 39^{\prime \prime} \\
\text { Long } 106^{\circ} 14^{\prime} 07^{\prime \prime}\end{array}$ & $m$ & 30.5 & 6.5 & $\rightarrow$ & -. & $0-1100$ & $\begin{array}{l}\text { Water-supply well drilled } 617 \mathrm{~m} \\
\text { Santa Fe Fm.; steel casing; Puye } \\
\text { Quadrangle }\end{array}$ & G-S; P79 \\
\hline 13 & G-5 Well & $L A-13$ & $9 / 78$ & $\begin{array}{l}\text { Lat } 35^{\circ} 54^{\prime} 51^{\prime \prime} \\
\text { Long } 106^{\circ} 13^{\circ} 37^{\prime \prime}\end{array}$ & $m$ & 26.5 & 6.5 & -- & -- & $0-2100$ & $\begin{array}{l}\text { Wa ter-supply well drilled } 614 \mathrm{~m} \\
\text { into Santa Fe Fm.; steel casing; } \\
\text { Puye Quadrangle }\end{array}$ & $\begin{array}{l}\text { G-S; Gr; } \\
\text { P79; P-C }\end{array}$ \\
\hline 14 & G-4 Hell & LA-14 & $9 / 78$ & $\begin{array}{l}\text { Lat } 35^{\circ} 54^{\prime} 35^{\prime \prime} \\
\text { Long } 106^{\circ} 13^{\prime} 14^{\prime \prime}\end{array}$ & $m$ & 26 & 6.5 & -- & -- & $0-1260$ & $\begin{array}{l}\text { Wa ter-supply well drilled } 616 \mathrm{~m} \\
\text { into Santa Fe Fm.; steel casing; } \\
\text { Puye Quadrangle }\end{array}$ & $\begin{array}{l}\text { G-S; Gr: } \\
\text { P79 }\end{array}$ \\
\hline 15 & G-3 Hell & $L A-15$ & $9 / 78$ & $\begin{array}{l}\text { Lat } 35^{\circ} 54^{\prime} 33^{\prime \prime} \\
\text { Long } 106^{\circ} 12^{\prime} 41^{\prime \prime}\end{array}$ & $m$ & 29 & 6.5 & -- & - & $0-1620$ & $\begin{array}{l}\text { Water-supply well drilled } 614 \mathrm{~m} \\
\text { into Santa Fe Fm.; steel casing; } \\
\text { Puye Quadrangle }\end{array}$ & G-S; Gr; P79 \\
\hline 16 & G-2 Well & $L A-16$ & $9 / 78$ & $\begin{array}{l}\text { Lat } 35^{\circ} 54^{\prime} 22^{\prime \prime} \\
\text { Long } 106^{\circ} 12^{\prime} 09^{n}\end{array}$ & $m$ & 30 & 6.5 & - & -- & $0-1820$ & $\begin{array}{l}\text { Wa ter-supply well drilled } 617 \mathrm{~m} \\
\text { into Santa Fe Fm.; steel casing; } \\
\text { Puye Quadrangle }\end{array}$ & G-S; Gr; P79 \\
\hline 17 & G-1A Well & LA-17 & $9 / 78$ & $\begin{array}{l}\text { Lat } 35^{\circ} 54^{\prime} 14^{\prime \prime} \\
\text { Long } 106^{\circ} 11^{\prime} 59^{\prime \prime}\end{array}$ & $m$ & 28 & 6.5 & -- & -- & $0-2060$ & $\begin{array}{l}\text { Water-supply well drilled } 637 \mathrm{~m} \\
\text { into Santa Fe Fm.; steel casing; } \\
\text { Puye Quadrangle }\end{array}$ & G-S; P79; \\
\hline 18 & G-1 Hell & $L A-18$ & $9 / 78$ & $\begin{array}{l}\text { Lat } 35^{\circ} 54^{\prime} 06^{\prime \prime} \\
\text { Long } 106^{\circ} 11^{\prime} 48^{\prime \prime}\end{array}$ & m & 26 & 6.5 & -- & -- & $0-1340$ & $\begin{array}{l}\text { Wa ter-supply well drilled } 646 \mathrm{~m} \\
\text { into Santa Fe Fm.; steel casing; } \\
\text { Puye Quadrangle }\end{array}$ & $\begin{array}{l}\text { G-S; Gr;P79 } \\
P-C\end{array}$ \\
\hline 19 & $\begin{array}{l}\text { Spring, white } \\
\text { Rock Canyon }\end{array}$ & LA-19 & $9 / 78$ & $\begin{array}{l}\text { Lat } 35^{\circ} 45^{\prime} 07^{n} \\
\text { Long } 106^{\circ} 15^{\prime} 21^{\prime \prime}\end{array}$ & m & 19 & 6.5 & -- & -- & 6 & $\begin{array}{l}\text { Spring issues from Santa Fe Fm.; } \\
\text { covered by volcanic colluvium; } \\
\text { Frijoles Quadrangle }\end{array}$ & $\begin{array}{l}\text { G-S; G et } \\
\text { al; T78 } \\
\left.(\mathrm{K} i)^{\circ}\right)\end{array}$ \\
\hline 20 & PM-3 Well & $\angle A-20$ & $9 / 78$ & $\begin{array}{l}\text { Lat } 35^{\circ} 51^{\prime} 47^{\prime \prime} \\
\text { Long } 106^{\circ} 14^{\prime} 41^{\prime \prime}\end{array}$ & m & 27.5 & 6.5 & -- & -- & $0-5600$ & $\begin{array}{l}\text { Well drilled } 786 \mathrm{~m} \text { into Santa Fe } \\
\text { Fm.; steel casing; White Rock } \\
\text { Quadrangle }\end{array}$ & G-S; P79 \\
\hline 32 & Pajarito Spring & VA-29 & $7 / 79$ & $\begin{array}{l}\text { Lat } 35^{\circ} 48^{\prime} 08^{\prime \prime} \\
\text { Long } 106^{\circ} 11^{\prime} 48^{\prime \prime}\end{array}$ & $\mathbf{m}$ & 20 & 5.9 & -- & 210 & $>300$ & $\begin{array}{l}\text { Spring issues from land- } \\
\text { slide in basalt; spring } 4 \mathrm{~A} \\
\text { of Purtyman; White Rock } \\
\text { Quadrangle }\end{array}$ & $\begin{array}{l}P-P-0 ; T 78 \\
(K 4)\end{array}$ \\
\hline 33 & $\begin{array}{l}\text { Spring, White } \\
\text { Rock Canyon }\end{array}$ & $V A-30$ & $7 / 79$ & $\begin{array}{l}\text { Lat } 35^{\circ} 48^{\prime} 15^{\prime \prime} \\
\text { Long } 106^{\circ} 11^{\prime} 40^{\prime \prime}\end{array}$ & $\mathrm{m}$ & 18 & 6.0 & -- & -- & 1 & $\begin{array}{l}\text { Spring issues from allu- } \\
\text { vium west of Rio Grande; } \\
\text { spring } 4 \text { of Purtyman; White } \\
\text { Rock Quadrangle }\end{array}$ & $\begin{array}{l}P-P-0 ; T 78 \\
(K 5)\end{array}$ \\
\hline
\end{tabular}




\begin{tabular}{|c|c|c|c|c|c|c|c|c|c|c|c|c|}
\hline $\begin{array}{l}\text { Map } \\
\text { No. }\end{array}$ & Name & $\begin{array}{l}\text { Field } \\
\text { No. }\end{array}$ & Date & Location & $\begin{array}{l}\text { Wa ter } \\
\text { Type }\end{array}$ & Temp & $\underset{\text { pH }}{\text { Field }}$ & $\begin{array}{l}\text { Field } \\
\text { Eh(mV) }\end{array}$ & $\begin{array}{l}\text { Conduc. } \\
\text { (unhos/cm) }\end{array}$ & $\begin{array}{c}\text { Flow } \\
\text { Rate } \\
(2 / \text { in })\end{array}$ & Comments & Reference $^{\mathrm{b}}$ \\
\hline 52 & $\begin{array}{l}\text { Unnamed cold } \\
\text { Spring }\end{array}$ & $V A-58$ & $7 / 80$ & $\begin{array}{l}\text { Lat } 35^{\circ} 48^{\prime} 17^{\prime \prime} \\
\text { Long } 106^{\circ} 19^{\prime} 55^{\prime \prime}\end{array}$ & $m$ & 15 & 5.4 & -- & 130 & 12 & $\begin{array}{l}\text { Spring flows from fractured } \\
\text { Bandelier Tuff; Frijoles } \\
\text { Quadrangle }\end{array}$ & \\
\hline 53 & $\begin{array}{l}\text { Unnamed cold } \\
\text { Spring }\end{array}$ & VA-59 & $7 / 80$ & $\begin{array}{l}\text { Lat } 35^{\circ} 48^{\circ} 18^{\prime \prime} \\
\text { Long } 106^{\circ} 20^{\prime} 12^{\prime \prime}\end{array}$ & $m$ & 17 & 5.6 & -- & 130 & 24 & $\begin{array}{l}\text { Spring issues from fractured } \\
\text { Bandelier Tuff; Frijoles } \\
\text { quadrangle }\end{array}$ & \\
\hline 28 & San Antonio Creek & $V A-24$ & $2 / 29$ & $\begin{array}{l}\text { Lat } 35^{\circ} 58^{\prime} 17^{\prime \prime} \\
\text { Long } 106^{\circ} 33^{\circ} 38^{\prime \prime}\end{array}$ & $m$ & 2 & -- & -- & -- & $>250$ & $\begin{array}{l}\text { Creek sampled } 5 \text { m upstream of } \\
\text { vA-20, Ba thhouse Spring; valle } \\
\text { San Antonio Quadrangle }\end{array}$ & \\
\hline 28 & San Antonio Creek & VA-95 & $3 / 82$ & $\begin{array}{l}\text { Lat } 35^{\circ} 58^{\circ} 17^{\prime \prime} \\
\text { Long } 106^{\circ} 33^{\prime} 38^{\prime \prime}\end{array}$ & $m$ & 3.0 & 7.41 & 386 & 124 & $>500$ & $\begin{array}{l}\text { Creek sampled } 5 \text { m upstream of } \\
\text { VA-20, Ba thhouse Spring; Valle } \\
\text { San Antonio Quadrangle. }\end{array}$ & \\
\hline 28 & San Antonio Creek & VA-104 & $8 / 82$ & $\begin{array}{l}\text { Lat } 35^{\circ} 58^{\prime} 17^{\prime \prime} \\
\text { Long } 106^{\circ} 33^{\prime} 38^{\prime \prime}\end{array}$ & m & 22.4 & -- & -- & 111 & -- & $\begin{array}{l}\text { Creek sampled } 5 \text { m upstream of } \\
\text { VA-20, Ba thhouse Spring; Valle } \\
\text { San Antonio Quadrangle }\end{array}$ & \\
\hline 28 & San Antonio Creek & VA-127 & $3 / 83$ & $\begin{array}{l}\text { Lat } 35^{\circ} 58^{\prime} 12^{\prime \prime} \\
\text { Long } 106^{\circ} 33^{\prime} 38^{\prime \prime}\end{array}$ & m & 0.5 & 7.36 & 112 & 123 & -- & $\begin{array}{l}\text { Creek sampled } 5 \mathrm{~m} \text { upstream of } \\
\text { VA-20, Bathhouse Spring; Valle } \\
\text { San Antonio Quadrangle }\end{array}$ & \\
\hline 29 & $\begin{array}{l}\text { Jemez River } \\
\text { at Soda Dam }\end{array}$ & VA-52 & $4 / 80$ & $\begin{array}{l}\text { Lat } 35^{\circ} 47^{\prime} 29^{\prime \prime} \\
\text { Long } 106^{\circ} 41^{\prime} 11^{\prime \prime}\end{array}$ & $m$ & 5 & 5.30 & -- & -- & $\gg>500$ & $\begin{array}{l}\text { River sampled } 10 \mathrm{~m} \text { upstream } \\
\text { of Soda Dam travertine deposit; } \\
\text { high water: Jemez Springs } \\
\text { Quadrangle }\end{array}$ & P-W-A; T78 \\
\hline 29 & $\begin{array}{l}\text { Jemez River } \\
\text { at Soda Dam }\end{array}$ & $V A-88$ & $3 / 82$ & $\begin{array}{l}\text { Lat } 35^{\circ} 47^{\prime} 29^{\prime \prime} \\
\text { Long } 106^{\circ} 41^{\prime} 11^{\prime \prime}\end{array}$ & $\pi$ & 1.9 & 6.62 & -- & 270 & $\gg>1000$ & $\begin{array}{l}\text { River sampled } 10 \mathrm{~m} \text { upstream } \\
\text { of Soda Dam travertine deposit; } \\
\text { high wa ter; Jemez Springs } \\
\text { Quadrangle }\end{array}$ & \\
\hline 29 & $\begin{array}{l}\text { Jemez River } \\
\text { at Soda Dam }\end{array}$ & VA-111 & $1 / 83$ & $\begin{array}{l}\text { Lat } 35^{\circ} 49^{\prime} 46^{\prime \prime} \\
\text { Long } 106^{\circ} 41^{\prime} 11^{\prime \prime}\end{array}$ & $m$ & 0.7 & 6.80 & 5 & 240 & 2000 & $\begin{array}{l}\text { River sampled } 10 \mathrm{~m} \text { upstream } \\
\text { of Soda Dam travertine deposit; } \\
\text { high wa ter; Jemez Springs } \\
\text { Quadrangle }\end{array}$ & \\
\hline 29 & $\begin{array}{l}\text { Jemez River at } \\
\text { Soda Dam }\end{array}$ & VA-131 & $5 / 83$ & $\begin{array}{l}\text { Lat } 35^{\circ} 47^{\prime} 29^{\prime \prime} \\
\text { Long } 106^{\circ} 41^{\prime} 11^{\prime \prime}\end{array}$ & m & 9 & 7.19 & $338^{c}$ & -- & -- & $\begin{array}{l}\text { River sampled } 10 \mathrm{~m} \text { upstream } \\
\text { of Soda Dam travertine deposit; } \\
\text { high wa ter; Jemez Springs } \\
\text { quadrangle }\end{array}$ & \\
\hline
\end{tabular}


TABLE B-I (cont)

\begin{tabular}{|c|c|c|c|c|c|c|c|c|c|c|c|c|}
\hline $\begin{array}{l}\text { Map } \\
\text { No. }\end{array}$ & Name & $\begin{array}{l}\text { Field } \\
\text { No. }\end{array}$ & Date & Location & $\begin{array}{l}\text { Wa ter } \\
\text { Type }\end{array}$ & $\begin{array}{l}\text { Temp } \\
(\times c)\end{array}$ & $\begin{array}{l}\text { Field } \\
\text { pH }\end{array}$ & $\begin{array}{l}\text { Field } \\
\text { Eh(mV) }\end{array}$ & $\begin{array}{l}\text { Conduc. } \\
\text { (mnhos/cm) }\end{array}$ & $\begin{array}{c}\text { Flow } \\
\text { Rate } \\
(\ell / \min )\end{array}$ & Comments & Reference $^{b}$ \\
\hline 31 & Panorama Spring & $V A-28$ & $5 / 79$ & $\begin{array}{l}\text { Lat } 35^{\circ} 48^{\prime} 13^{\prime \prime} \\
\text { Long } 105^{\circ} 41^{\prime} 10^{\prime \prime}\end{array}$ & m & 13 & 7.67 & -- & 920 & 4 & $\begin{array}{l}\text { Spring issues from travertine } \\
\text { and Madera limestone, } 100 \mathrm{~m} \\
\text { above canyon floor; Jemez Springs } \\
\text { Quadrangle }\end{array}$ & \\
\hline 31 & Panorama Spring & VA-86 & $3 / 82$ & $\begin{array}{l}\text { Lat } 35^{\circ} 48^{\circ} 13^{\prime \prime} \\
\text { Long } 106^{\circ} 41^{\prime} 10^{\prime \prime}\end{array}$ & $m$ & 7.2 & 7.87 & -- & 737 & 4 & $\begin{array}{l}\text { Spring issues from travertine } \\
\text { and Madera limestone, } 100 \mathrm{~m} \\
\text { above canyon floor; Jemez Springs } \\
\text { Quadrangle }\end{array}$ & \\
\hline 31 & $\begin{array}{c}\text { Panorama Spring } \\
\vdots\end{array}$ & $V A-136$ & $5 / 83$ & $\begin{array}{l}\text { Lat } 35^{\circ} 48^{\prime} 13^{\prime \prime} \\
\text { Long } 106^{\circ} 41^{\prime} 10^{\prime \prime}\end{array}$ & $m$ & 9 & 8.00 & $368^{c}$ & 810 & -- & $\begin{array}{l}\text { Spring issues from travertine } \\
\text { and Madera limestone, } 100 \mathrm{~m} \\
\text { above canyon floor; Jemez Springs } \\
\text { Quadrangle }\end{array}$ & \\
\hline 35 & Sino Spring & VA-32 & $8 / 79$ & $\begin{array}{l}\text { Lat } 35^{\circ} 49^{\prime} 17^{\prime \prime} \\
\text { Long } 106^{\circ} 40^{\prime} 42^{\prime \prime}\end{array}$ & m & 21 & 7.45 & -- & -- & 15 & $\begin{array}{l}\text { Spring flows from contact of } \\
\text { andesite and Abo Formation; } \\
\text { Jemez Springs Quadrangle }\end{array}$ & $\begin{array}{l}\text { P-H-A; T78 } \\
\text { (H1) }\end{array}$ \\
\hline 35 & Sino Spring & VA-63 & $12 / 80$ & $\begin{array}{l}\text { Lat } 35^{\circ} 49^{\circ} 17^{\prime \prime} \\
\text { Long } 106^{\circ} 40^{\prime} 42^{\prime \prime}\end{array}$ & $m$ & 18 & 7.10 & -- & 163 & 80 & $\begin{array}{l}\text { Spring flows from contact of } \\
\text { andesite and Abo Formation; } \\
\text { Jemez Springs Quadrangle }\end{array}$ & \\
\hline 35 & Sino Spring & $V A-69$ & $6 / 81$ & $\begin{array}{l}\text { Lat } 35^{\circ} 49^{\circ} 17^{\prime \prime} \\
\text { Long } 106^{\circ} 40^{\prime} 42^{\prime \prime}\end{array}$ & $m$ & 22 & 7.77 & 290 & 160 & 80 & $\begin{array}{l}\text { Spring flows from contact of } \\
\text { andesite and Abo Formation; } \\
\text { Jemez Springs Quadrangle }\end{array}$ & \\
\hline 35 & Sino Spring & $V A-85$ & $3 / 82$ & $\begin{array}{l}\text { Lat } 35^{\circ} 49^{\circ} 17^{\prime \prime} \\
\text { Long } 106^{\circ} 40^{\prime} 42^{\prime \prime}\end{array}$ & $\mathrm{m}$ & 16.9 & 7.68 & -- & 172 & 80 & $\begin{array}{l}\text { Spring flows from contact of } \\
\text { andesite and Abo Formation; } \\
\text { Jemez Springs Quadrangle }\end{array}$ & \\
\hline 35 & Sino Spring & YA-102 & $8 / 82$ & $\begin{array}{l}\text { Lat } 35^{\circ} 49^{\circ} 17^{\prime \prime} \\
\text { Long } 106^{\circ} 40^{\prime} 42^{n}\end{array}$ & $m$ & 21.3 & -- & -- & 150 & -- & $\begin{array}{l}\text { Spring flows from contact of } \\
\text { andesite and Abo Formation; } \\
\text { Jemez Springs Quadrangle }\end{array}$ & \\
\hline 38 & $\begin{array}{l}\text { Indian valley } \\
\text { Well }\end{array}$ & $V A-41$ & $10 / 79$ & $\begin{array}{l}\text { Lat } 35^{\circ} 48^{\prime} 16^{\prime \prime} \\
\text { Long } 106^{\circ} 35^{\prime} 10^{\prime \prime}\end{array}$ & $\mathbf{m}$ & 17.5 & 6.81 & -- & 280 & 0 & $\begin{array}{l}\text { Well drilled } 6+m \text { in allu- } \\
\text { vium and pumice; iron casing; } \\
\text { Redondo Peak Quadrangle }\end{array}$ & \\
\hline 40 & Unnamed Spring & VA-43 & $10 / 79$ & $\begin{array}{l}\text { Lat } 35^{\circ} 48^{\prime} 26^{\prime \prime} \\
\text { Long } 106^{\circ} 30^{\prime} 30^{\prime \prime}\end{array}$ & m & 15 & 6.68 & 240 & 65 & 8 & $\begin{array}{l}\text { Spring flows from dacite } \\
\text { colluvium; Redondo Peak } \\
\text { Quadrangle }\end{array}$ & \\
\hline 40 & Unnamed Spring & VA-139 & $5 / 83$ & $\begin{array}{l}\text { Lat } 35^{\circ} 48^{\prime} 26^{\prime \prime} \\
\text { Long } 106^{\circ} 30^{\prime} 30^{\prime \prime}\end{array}$ & $m$ & 3 & 6.98 & $411^{c}$ & 85 & -- & $\begin{array}{l}\text { Spring flows from dacite } \\
\text { colluvium; Redondo Peak } \\
\text { Quadrangle }\end{array}$ & \\
\hline
\end{tabular}




\begin{tabular}{|c|c|c|c|c|c|c|c|c|c|c|c|c|}
\hline $\begin{array}{l}\text { Map } \\
\text { No. }\end{array}$ & Name & $\begin{array}{l}\text { Field } \\
\text { No. }\end{array}$ & Date & Location & $\begin{array}{l}\text { Wa ter } \\
\text { Type }\end{array}$ & $\begin{array}{l}\text { Temp } \\
\left({ }^{\circ} \mathrm{C}\right)\end{array}$ & $\begin{array}{l}\text { Field } \\
\text { pH }\end{array}$ & $\begin{array}{l}\text { Field } \\
\text { Eh(my) }\end{array}$ & $\begin{array}{l}\text { Conduc. } \\
(\text { umhos } / \mathrm{cm})\end{array}$ & $\begin{array}{c}\text { Flow } \\
\text { Rate } \\
(\ell / \min )\end{array}$ & Comments & Reference ${ }^{b}$ \\
\hline 41 & $\begin{array}{l}\text { Unnamed cold } \\
\text { Spring }\end{array}$ & $V A-44$ & $10 / 79$ & $\begin{array}{l}\text { Lat } 35^{\circ} 46^{\prime} 36^{\prime \prime} \\
\text { Long } 106^{\circ} 36^{\prime} 14^{\prime \prime}\end{array}$ & $m$ & 8 & 6.91 & -- & 210 & 2 & $\begin{array}{l}\text { Spring discharges from } \\
\text { rock crib on andesite } \\
\text { alluvium by dirt road; } \\
\text { Redondo Peak Quadrangle }\end{array}$ & $T 78$ (J2) \\
\hline 43 & $\begin{array}{l}\text { Unnamed Cold } \\
\text { Spring }\end{array}$ & $V A-46$ & $10 / 79$ & $\begin{array}{l}\text { Lat } 35^{\circ} 52^{\prime} 33^{\prime \prime} \\
\text { Long } 106^{\circ} 44^{\prime} 37^{\prime \prime}\end{array}$ & m & 10 & 6.42 & -- & 126 & 40 & $\begin{array}{l}\text { Spring flows from contact } \\
\text { of Bandelier Tuff and Abo } \\
\text { Fm.; sampled from iron pipe; } \\
\text { Seven Springs Quadrangle }\end{array}$ & T78 (N6) \\
\hline 44 & $\begin{array}{l}\text { Seven Springs } \\
\text { Spring }\end{array}$ & $V A-47$ & $10 / 79$ & $\begin{array}{l}\text { Lat } 35^{\circ} 56^{\prime} 35^{\prime \prime} \\
\text { Long } 106^{\circ} 42^{\prime} 15^{\prime \prime}\end{array}$ & $m$ & 10 & 6.48 & -- & 115 & 60 & $\begin{array}{l}\text { Spring discharges from } \\
\text { fractured Bandelier Tuff; } \\
\text { Seven Springs Quadrangle }\end{array}$ & $T 78$ (N10) \\
\hline 44 & $\begin{array}{l}\text { Seven Springs } \\
\text { Spring }\end{array}$ & $V A-138$ & $5 / 83$ & $\begin{array}{l}\text { Lat } 35^{\circ} 56^{\prime} 35^{\prime \prime} \\
\text { Long } 106^{\circ} 42^{\prime} 15^{\prime \prime}\end{array}$ & $m$ & 12 & 7.41 & $382^{c}$ & 112 & -- & $\begin{array}{l}\text { Spring discharges from } \\
\text { fractured Bandelier Tuff; } \\
\text { Seven Springs Quadrangle }\end{array}$ & T78 (N10) \\
\hline 44 & $\begin{array}{l}\text { Cold Spring West } \\
\text { of Caldera }\end{array}$ & $V A-134$ & $5 / 83$ & $\begin{array}{l}\text { Lat } 35^{\circ} 56^{\prime} 35^{\prime \prime} \\
\text { Long } 106^{\circ} 42^{\prime} 15^{\prime \prime}\end{array}$ & m & 12 & 7.45 & $376^{c}$ & 115 & -- & $\begin{array}{l}\text { Spring discharges from } \\
\text { fractured Bandelier Tuff; } \\
\text { Seven Springs Quadrangle }\end{array}$ & \\
\hline 45 & $\begin{array}{l}\text { Unnamed cold } \\
\text { Spring }\end{array}$ & $V A-48$ & $6 / 80$ & $\begin{array}{l}\text { Lat } 35^{\circ} 44^{\prime} 47^{\prime \prime} \\
\text { Long } 106^{\circ} 28^{\prime} 55^{\prime \prime}\end{array}$ & m & 9 & 5.6 & -- & 280 & 12 & $\begin{array}{l}7 \text { Springs issue from gully } \\
1 / 2 \mathrm{~km} \text { east at Albermarle } \\
\text { ruins; from hypabyssal volcanics; } \\
\text { Cañada Quadrangle }\end{array}$ & \\
\hline 46 & Eddy's Well & $V A-49$ & $6 / 80$ & $\begin{array}{l}\text { Lat } 35^{\circ} 52^{\prime} 44^{\prime \prime} \\
\text { Long } 106^{\circ} 37^{\prime} 28^{\prime \prime}\end{array}$ & $m$ & 15 & 7.24 & -60 & 550 & $0-20$ & $\begin{array}{l}\text { Well } 40+m \text { deep in alluvium } \\
\text { and pumice; Valle San Antonio } \\
\text { Quadrangle }\end{array}$ & \\
\hline 46 & Henson's Well & $V A-61$ & $12 / 80$ & $\begin{array}{l}\text { Lat } 35^{\circ} 52^{\prime} 44^{\prime \prime} \\
\text { Long } 106^{\circ} 37^{\circ} 28^{\prime \prime}\end{array}$ & $m$ & 19 & 6.82 & -- & 560 & $0-40$ & $\begin{array}{l}\text { Well drilled } 65 \mathrm{~m} \text { into caldera } \\
\text { fill; plastic pipe; valle San } \\
\text { Antonio Quadrangle }\end{array}$ & \\
\hline 48 & $\begin{array}{l}\text { Unnamed cold } \\
\text { Spring }\end{array}$ & $V A-54$ & $6 / 80$ & $\begin{array}{l}\text { Lat } 35^{\circ} 47^{\prime} 50^{\prime \prime} \\
\text { Long } 106^{\circ} 28^{\prime} 08^{\prime \prime}\end{array}$ & $m$ & 11 & 5.4 & -- & 89 & 20 & $\begin{array}{l}\text { Spring issues from alluvium } \\
\text { in Cânon del Norte; Bland } \\
\text { Quadrangle }\end{array}$ & \\
\hline 49 & $\begin{array}{l}\text { Unnamed cold } \\
\text { Spring }\end{array}$ & $V A-55$ & $6 / 80$ & $\begin{array}{l}\text { Lat } 35^{\circ} 50^{\prime} 29^{\prime \prime} \\
\text { Long } 106^{\circ} 25^{\prime} 25^{\prime \prime}\end{array}$ & m & 8.5 & 5.4 & -- & 55 & 20 & $\begin{array}{l}\text { Spring issues from contact } \\
\text { of latite and Bandelier Tuff; } \\
\text { Bland Quadrangle }\end{array}$ & \\
\hline 50 & $\begin{array}{l}\text { Unnamed Cold } \\
\text { Spring }\end{array}$ & $V A-56$ & $6 / 80$ & $\begin{array}{l}\text { Lat } 35^{\circ} 50^{\prime} 07^{\prime \prime} \\
\text { Long } 106^{\circ} 24^{\prime} 42^{\prime \prime}\end{array}$ & $m$ & 6.5 & 5.4 & -- & 105 & 24 & $\begin{array}{l}\text { Spring discharges from contact } \\
\text { of latite and Bandelier Tuff: } \\
\text { Bland Quadrangle }\end{array}$ & \\
\hline
\end{tabular}


TABLE B-I (cont)

\begin{tabular}{|c|c|c|c|c|c|c|c|c|c|c|c|c|}
\hline $\begin{array}{l}\text { Map } \\
\text { No. }\end{array}$ & Name & $\begin{array}{l}\text { Field } \\
\text { No. }\end{array}$ & Date & Location & $\begin{array}{l}\text { Wa ter } \\
\text { Type }\end{array}$ & $\left({ }^{\top} \mathrm{Cmp}\right.$ & $\underset{\mathrm{pH}}{\mathrm{Ffeld}}$ & $\begin{array}{l}\text { Field } \\
\text { Eh(mV) }\end{array}$ & $\begin{array}{l}\text { Conduc. } \\
\text { (umhos/cm) }\end{array}$ & $\begin{array}{l}\text { Flow } \\
\text { Rate } \\
(\Omega / m i n)\end{array}$ & Comnents & Reference $^{b}$ \\
\hline 51 & Apache Spring & YA-57 & $7 / 80$ & $\begin{array}{l}\text { Lat } 35^{\circ} 49^{\prime} 30^{\prime \prime} \\
\text { Long } 106^{\circ} 23^{\prime} 31^{\prime \prime}\end{array}$ & m & 9 & 5.3 & -- & 137 & 15 & $\begin{array}{l}\text { Spring discharges from contact } \\
\text { of la tite and Bandelier Tuff: } \\
\text { Bland Quadrangle. }\end{array}$ & \\
\hline 54 & Turkey Spring & $V A-60$ & $7 / 80$ & $\begin{array}{l}\text { Lat } 35^{\circ} 44^{\prime} 23^{\prime \prime} \\
\text { Long } 106^{\circ} 21^{\prime} 35^{\prime \prime}\end{array}$ & m & 18 & 5.5 & -- & 193 & 60 & $\begin{array}{l}\text { Spring flows from volcanic } \\
\text { alluvium; Cochiti Dan Quadrangle }\end{array}$ & \\
\hline 54 & Turkey Spring & $V A-137$ & $5 / 83$ & $\begin{array}{l}\text { Lat } 35^{\circ} 44^{\prime} 23^{\prime \prime} \\
\text { Long } 106^{\circ} 21^{\prime} 35^{\prime \prime}\end{array}$ & $m$ & 18 & 7.67 & $363^{c}$ & 196 & -- & $\begin{array}{l}\text { Spring flows from volcanic } \\
\text { alluvium; Cochiti Dam Quadrangle }\end{array}$ & \\
\hline 68 & $\begin{array}{l}\text { Las Conchas } \\
\text { Spring }\end{array}$ & $V A-124$ & $1 / 84$ & $\begin{array}{l}\text { Lat } 35^{\circ} 49^{\circ} 16^{\prime \prime} \\
\text { Long } 106^{\circ} 32^{\prime} 08^{\prime \prime}\end{array}$ & m & 14 & 6.75 & 344 & 95 & $>40$ & Redondo Peak Quadrangle & \\
\hline \multicolumn{13}{|c|}{ Valles Caldera - Sulphur Springs Area } \\
\hline 21 & $\begin{array}{l}\text { Mudpot, Men's } \\
\text { Ba thhouse }\end{array}$ & VA-13 & $1 / 79$ & $\begin{array}{l}\text { Lat } 35^{\circ} 54^{\prime} 29^{\prime \prime} \\
\text { Long } 106^{\circ} 36^{\prime} 54^{\prime \prime}\end{array}$ & a & 78 & 2.52 & - & 4050 & $\mathbf{0}$ & $\begin{array}{l}\text { Mudpot in concrete crib in col- } \\
\text { lapsed bathhouse. From landslide } \\
\text { in rhyolite; } \mathrm{CO}_{2}+\mathrm{H}_{2} \mathrm{St} \text {; Valle } \\
\text { San Antonio Quadrangfe }\end{array}$ & $\begin{array}{l}\text { G-G; S; R; } \\
\text { T78(P2) }\end{array}$ \\
\hline 21 & $\begin{array}{l}\text { Mudpot, Men's } \\
\text { Ba thhouse }\end{array}$ & $s-7-80$ & $9 / 80$ & $\begin{array}{l}\text { Lat } 35^{\circ} 54^{\prime} 29^{\prime \prime} \\
\text { Long } 106^{\circ} 36^{\prime} 54^{\prime \prime}\end{array}$ & $\mathbf{a}$ & 82 & 2.0 & -- & 10300 & 0 & $\begin{array}{l}\text { Mudpot in concrete crib in col- } \\
\text { lapsed bathhouse. From landslide } \\
\text { in rhyolite; } \mathrm{CO}_{2}+\mathrm{H}_{2} \mathrm{St} \text { V Valle } \\
\text { San Antonio Quaurangfe }\end{array}$ & \\
\hline 21 & $\begin{array}{l}\text { Mudpot, Men's } \\
\text { Ba thhouse }\end{array}$ & VA-75 & $1 / 82$ & $\begin{array}{l}\text { Lat } 35^{\circ} 54^{\prime} 29^{\prime \prime} \\
\text { Long } 106^{\circ} 36^{\prime} 54^{\prime \prime}\end{array}$ & $\mathbf{a}$ & 72 & $<2.5$ & 11.85 & -- & 0 & $\begin{array}{l}\text { Mudpot in concrete crib in col- } \\
\text { lapsed bathhouse. From landslide } \\
\text { in rhyolite; } \mathrm{CO}_{2}+\mathrm{H}_{2} \mathrm{~S}_{4} \text {; Valle } \\
\text { San Antonio Quadrangle }\end{array}$ & \\
\hline 21 & $\begin{array}{l}\text { Steam, Men's } \\
\text { Bathhouse }\end{array}$ & $V A-81$ & $3 / 82$ & $\begin{array}{l}\text { Lat } 35^{\circ} 54^{\prime} 29^{\prime \prime} \\
\text { Long } 106^{\circ} 36^{\prime} 54^{\prime \prime}\end{array}$ & a & 73.6 & 2.88 & 169 & 1300 & -- & $\begin{array}{l}\text { Mudpot in concrete crib in col- } \\
\text { lapsed bathhouse. From landslide } \\
\text { in rhyolite; } \mathrm{CO}_{2}^{+} \text {, } \mathrm{H}_{2} \mathrm{St}^{+} \text {Valle } \\
\text { San Antonio Quadrangle }\end{array}$ & \\
\hline 21 & Women's Ba thhouse & $S-6-80$ & $3 / 82$ & $\begin{array}{l}\text { Lat } 35^{\circ} 54^{\prime} 29^{\prime \prime} \\
\text { Long } 106^{\circ} 36^{\prime} 54^{\prime \prime}\end{array}$ & a & 88.0 & 4.30 & 219 & 12800 & -- & $\begin{array}{l}\text { Spring issues from landslide; } \\
\mathrm{CO}_{2}^{4}, \mathrm{H}_{2} \mathrm{St} \text { : Valle San Antonio } \\
\text { Quadrangle }\end{array}$ & \\
\hline 21 & Women's Bathhouse & $V A-76$ & $1 / 82$ & $\begin{array}{l}\text { Lat } 35^{\circ} 54^{\prime} 29^{\prime \prime} \\
\text { Long } 106^{\circ} 36^{\prime} 54^{\prime \prime}\end{array}$ & a & 89 & $<2.5$ & -- & -- & $1 / 4$ & $\begin{array}{l}\text { Spring issues from landslide; } \\
\mathrm{CO}_{2}+\mathrm{H}_{2} \mathrm{St} ; \text { Valle San Antonio } \\
\text { Quadrangle }\end{array}$ & \\
\hline 21 & Footbath Springs & $S-4-80$ & $9 / 80$ & $\begin{array}{l}\text { Lat } 35^{\circ} 54^{\prime} 29^{\prime \prime} \\
\text { Long } 106^{\circ} 36^{\prime} 54^{\prime \prime}\end{array}$ & a & 33 & 1.1 & -- & 30200 & 0 & $\begin{array}{l}\text { Large bubbling pool with colloidal } \\
\text { sulfur: } \mathrm{CO}_{2}+\mathrm{H}_{2} \mathrm{St} \text {; Valle San Antor } \\
\text { Quadrangle }\end{array}$ & nio \\
\hline
\end{tabular}




\begin{tabular}{|c|c|c|c|c|c|c|c|c|c|c|c|c|}
\hline $\begin{array}{l}\text { Map } \\
\text { No. }\end{array}$ & Name & $\begin{array}{l}\text { Field } \\
\text { No. }\end{array}$ & Date & Location & $\begin{array}{l}\text { Water } \\
\text { Type }\end{array}$ & Temp & $\begin{array}{c}\text { Field } \\
\text { pH }\end{array}$ & $\begin{array}{l}\text { Field } \\
\text { Eh }(m V)\end{array}$ & $\begin{array}{l}\text { Conduc. } \\
(\text { umhos } / \mathrm{cm})\end{array}$ & $\begin{array}{l}\text { Flow } \\
\text { Rate } \\
(\ell / m i n)\end{array}$ & Comments & Reference $^{b}$ \\
\hline 21 & Footbath Springs & VA-77 & $1 / 82$ & $\begin{array}{l}\text { Lat } 35^{\circ} 54^{\prime} 29^{\prime \prime} \\
\text { Long } 106^{\circ} 36^{\prime} 54^{\prime \prime}\end{array}$ & a & 18 & $<2.5$ & -- & -- & $1 / 4$ & $\begin{array}{l}\text { Large bubbling pool with colloidal } \\
\text { sulfur: } \mathrm{CO}_{2}^{+}, \mathrm{H}_{2} \mathrm{St} \text { : Valle San } \\
\text { Antonio Quadrangle }\end{array}$ & \\
\hline 21 & Footbath Springs & VA-79 & $3 / 82$ & $\begin{array}{l}\text { Lat } 35^{\circ} 54^{\prime} 29^{\prime \prime} \\
\text { Long } 106^{\circ} 36^{\prime} 54^{\prime \prime}\end{array}$ & a & 14.6 & 1.95 & -- & 18400 & $1 / 4$ & $\begin{array}{l}\text { Large bubbling pool with colloidal } \\
\text { sulfur; } \mathrm{CO}_{2}^{+}, \mathrm{H}_{2} \mathrm{St} \text {; Valle San } \\
\text { Antonio Quadrangle }\end{array}$ & \\
\hline 21 & $\begin{array}{l}\text { Unnamed } \\
\text { Spring }\end{array}$ & $S-3-80$ & $9 / 80$ & $\begin{array}{l}\text { Lat } 35^{\circ} 54^{\prime} 29^{\prime \prime} \\
\text { Long } 106^{\circ} 36^{\prime} 54^{\prime \prime}\end{array}$ & $\mathrm{cm}$ & 11 & 3.6 & - & 1910 & $1 / 4$ & $\begin{array}{l}\text { Spring discharges into sulphur } \\
\text { Creek; } \mathrm{CO}_{2}^{+}, \mathrm{H}_{2} \mathrm{St;}^{+} \text {Valle San } \\
\text { Antonio Quadrangle }\end{array}$ & \\
\hline 21 & $\begin{array}{l}\text { Unnamed } \\
\text { Hot Spring }\end{array}$ & $S-9-80$ & $9 / 80$ & $\begin{array}{l}\text { Lat } 35^{\circ} 54^{\prime} 29^{\prime \prime} \\
\text { Long } 106^{\circ} 36^{\prime} 54^{\prime \prime}\end{array}$ & a & -- & 2.03 & -- & & 1 & $\begin{array}{l}\text { Spring issues from alluvium; } \\
\mathrm{CO}_{2}+, \mathrm{H}_{2} \mathrm{St} \text {; Valle San } \\
\text { Antonio Quadrangle }\end{array}$ & \\
\hline 21 & $\begin{array}{l}\text { Unnamed } \\
\text { Hot Spring }\end{array}$ & VA-14 & $1 / 79$ & $\begin{array}{l}\text { Lat } 35^{\circ} 54^{\prime} 29^{\prime \prime} \\
\text { Long } 106^{\circ} 36^{\prime} 54^{\prime \prime}\end{array}$ & a & 63 & 2.38 & -- & 5800 & 2 & $\begin{array}{l}\text { Spring issues from alluvium; } \\
\mathrm{CO}_{2}{ }^{4}, \mathrm{H}_{2} \mathrm{St} ; \text { Valle San } \\
\text { Antonio Quadrangle }\end{array}$ & $\mathbf{G}-\mathbf{G}$ \\
\hline 21 & Electric Spring & $S-5-80$ & $9 / 80$ & $\begin{array}{l}\text { Lat } 35^{\circ} 54^{\prime} 29^{\prime \prime} \\
\text { Long } 106^{\circ} 36^{\prime} 54^{\prime \prime}\end{array}$ & a & 36 & 1.5 & -- & 12800 & $1 / 2$ & $\begin{array}{l}\text { Spring flows from alluvium; } \\
\mathrm{CO}_{2}^{4}, \mathrm{H}_{2} \mathrm{St} \text {; Valle San } \\
\text { Antonio Quadrangle }\end{array}$ & $S$ \\
\hline 21 & Lemonade Spring & $s-10-80$ & $9 / 80$ & $\begin{array}{l}\text { Lat } 35^{\circ} 54^{\prime} 29^{\prime \prime} \\
\text { Long } 106^{\circ} 36^{\prime} 54^{\prime \prime}\end{array}$ & a & 58 & 2.3 & -- & - & $1 / 2$ & $\begin{array}{l}\text { Spring issues from alluvium; } \\
\mathrm{CO}_{2}^{+}, \mathrm{H}_{2} \mathrm{St;} \text { Valle San } \\
\text { Antonio Quadrangle }\end{array}$ & $S ; T 78$ (P1) \\
\hline 21 & Suiphur Creek & $V A-78$ & $1 / 82$ & $\begin{array}{l}\text { Lat } 35^{\circ} 54^{\prime} 29^{\prime \prime} \\
\text { Long } 106^{\circ} 36^{\prime} 54^{\prime \prime}\end{array}$ & $m$ & 0.5 & 6.0 & -- & -- & $>50$ & $\begin{array}{l}\text { Collected near Bill Mondt's } \\
\text { summer home; isotope only }\end{array}$ & \\
\hline 21 & $\begin{array}{l}\text { Steam, Ma in } \\
\text { Fumarole }\end{array}$ & $V A-80$ & $3 / 82$ & $\begin{array}{l}\text { Lat } 35^{\circ} 54^{\prime} 29^{\prime \prime} \\
\text { Long } 106^{\circ} 36^{\prime} 54^{\prime \prime}\end{array}$ & a & 88.0 & 4.30 & $219^{C}$ & 70 & -- & $\begin{array}{l}\text { Main fumarole } 25 \mathrm{~m} \text { north of } \\
\text { Men's Bathhouse Mudpot; from } \\
\text { landslide }\end{array}$ & G et al. \\
\hline 22 & $\begin{array}{l}\text { Spring, } \\
\text { Alamo Canyon }\end{array}$ & $S-1-80$ & $9 / 80$ & $\begin{array}{l}\text { Lat } 35^{\circ} 55^{\prime} 04^{\prime \prime} \\
\text { Long } 106^{\circ} 35^{\prime} 53^{\prime \prime}\end{array}$ & $\mathrm{cm}$ & 11 & 4.2 & 430 & 0 & -- & $\begin{array}{l}\text { Gaseous spring in alluvium } \\
\text { and creek bed; } \mathrm{CO}_{2}+\mathrm{H}_{2} \mathrm{~S}+; \\
\text { valle San Antonio Quadrangle }\end{array}$ & s \\
\hline 22 & $\begin{array}{l}\text { Creek, } \\
\text { Alamo Canyon }\end{array}$ & $S-2-80$ & $9 / 80$ & $\begin{array}{l}\text { Lat } 35^{\circ} 55^{\prime} 04^{\prime \prime} \\
\text { Long } 106^{\circ} 35^{\prime} 53^{\prime \prime}\end{array}$ & $\mathrm{cm}$ & 11 & 3.1 & -- & 340 & 4 & $\begin{array}{l}\text { Creek sampled } 5 m \text { upstream of } \\
\text { S-1-80; Valle San Antonio } \\
\text { Quadrangle }\end{array}$ & \\
\hline 22 & Bubbling Seep & $V A-23$ & $3 / 79$ & $\begin{array}{l}\text { Lat } 35^{\circ} 55^{\prime} 04^{\prime \prime} \\
\text { Long } 106^{\circ} 35^{\prime} 53^{\prime \prime}\end{array}$ & $\mathrm{cm}$ & 7 & 5.2 & -- & 730 & 1 & $\begin{array}{l}\text { Bubbling seep } 30 \mathrm{~m} \text { north of } \\
\mathrm{S}-1-80 \text {; from alluvium; } \\
\mathrm{CO}_{2}^{+}, \mathrm{H}_{2} \mathrm{~S}^{+} \text {; Valle San Antonio } \\
\text { Quadrangle }\end{array}$ & \\
\hline
\end{tabular}


TABLE B-I (cont)

\begin{tabular}{|c|c|c|c|c|c|c|c|c|c|c|c|c|}
\hline $\begin{array}{l}\text { Map } \\
\text { No. }\end{array}$ & Name & $\begin{array}{l}\text { Field } \\
\text { No. }\end{array}$ & Date & Location & $\begin{array}{l}\text { Water } \\
\text { Type }\end{array}$ & $\begin{array}{l}\text { Temp } \\
\left({ }^{\circ} \mathrm{c}\right)\end{array}$ & $\begin{array}{c}\text { Field } \\
\text { PH }\end{array}$ & $\begin{array}{c}\text { Field } \\
\text { Eh(mV) }\end{array}$ & $\begin{array}{l}\text { Conduc. } \\
\text { (umhos } / \mathrm{cm})\end{array}$ & $\begin{array}{c}\text { Flow } \\
\text { Rate } \\
(e / m i n)\end{array}$ & Comments & Reference $^{b}$ \\
\hline 23 & Bubbling Pool & VA-22 & $3 / 79$ & $\begin{array}{l}\text { Lat } 35^{\circ} 55^{\prime} 01^{\prime \prime} \\
\text { Long } 106^{\circ} 35^{\prime} 39^{\prime \prime}\end{array}$ & $\mathrm{Cm}$ & 0.5 & 4.5 & -- & 280 & 8 & $\begin{array}{l}\text { Large bubbling pond } 1 / 2 \mathrm{~km} \\
\text { east of } \mathrm{S}-1-80 ; \mathrm{CO}_{2}+\mathrm{H}_{2} \mathrm{~S}^{4} \text {; } \\
\text { valle San Antonio Quadrangle }\end{array}$ & $\begin{array}{l}G-G ; ; 78 \\
(P 19)\end{array}$ \\
\hline 24 & $\begin{array}{l}\text { Spring, } \\
\text { Short Canyon }\end{array}$ & $S-8-80$ & $9 / 80$ & $\begin{array}{l}\text { Lat } 35^{\circ} 54^{\prime} 52^{\prime \prime} \\
\text { Long } 106^{\circ} 36^{\prime} 07^{\prime \prime}\end{array}$ & $\mathrm{cm}$ & 8 & 4.1 & -- & 500 & 1. & $\begin{array}{l}\text { Ga seous spring issues from } \\
\text { all uvium; } \mathrm{CO}_{2}^{+}, \mathrm{H}_{2} \mathrm{St} \text { : valle } \\
\text { San Antonio Quadrangle }\end{array}$ & \\
\hline 63 & GRI Mudpit & $V A-107$ & $12 / 82$ & $\begin{array}{l}\text { Lat } 35^{\circ} 54^{\prime} 34^{\prime \prime} \\
\text { Long } 106^{\circ} 37^{\prime} 53^{\prime \prime}\end{array}$ & m & 1.5 & 9.80 & 160 & 2500 & -- & $\begin{array}{l}\text { Sample collected from hole } \\
\text { chipped through frozen mudpit; } \\
\text { Seven Springs Quadrangle }\end{array}$ & \\
\hline 63 & $\begin{array}{l}\text { GRI Well } \\
\text { (Well lhead) }\end{array}$ & VA-108 & $12 / 82$ & $\begin{array}{l}\text { Lat } 35^{\circ} 54^{\prime} 34^{\prime \prime} \\
\text { Long } 106^{\circ} 37^{\prime} 53^{\prime \prime}\end{array}$ & $m$ & -4 & -- & -- & -- & -- & $\begin{array}{l}\text { Gas sample collected from valve } \\
\text { at wellhead; Seven Springs } \\
\text { quadrangle }\end{array}$ & \\
\hline 63 & $\begin{array}{l}\text { GRI Nell at } \\
4800 \mathrm{ft}\end{array}$ & VA-113 & $1 / 83$ & $\begin{array}{l}\text { Lat } 35^{\circ} 54^{\circ} 34^{\prime \prime} \\
\text { Long } 105^{\circ} 37^{\prime} 53^{\prime \prime}\end{array}$ & m & 214 & 8.03 & -60 & 13720 & -- & $\begin{array}{l}\text { Sample collected from downhole } \\
\text { sampling device; Seven Springs } \\
\text { Quadrangle }\end{array}$ & \\
\hline 63 & $\begin{array}{l}\text { GRI Well at } \\
4800 \mathrm{ft}\end{array}$ & VA-114 & $1 / 83$ & $\begin{array}{l}\text { Lat } 35^{\circ} 54^{\circ} 34^{* \prime} \\
\text { Long } 106^{\circ} 37^{\prime} 53^{\prime \prime}\end{array}$ & $m$ & 214 & 6.92 & -125 & -- & -- & $\begin{array}{l}\text { Sample collected from downhole } \\
\text { sampling device; Seven Springs } \\
\text { Quadrangle }\end{array}$ & \\
\hline 63 & $\begin{array}{l}\text { GRI Kell } \\
\text { (Hell head) }\end{array}$ & VA-115 & $1 / 83$ & $\begin{array}{l}\text { Lat } 35^{\circ} 54^{\prime} 34^{\prime \prime} \\
\text { Long } 106^{\circ} 37^{\prime} 53^{\prime \prime}\end{array}$ & $\mathbf{m}$ & -- & - & - & -- & $\therefore$ & $\begin{array}{l}\text { Gas sample collected from valve } \\
\text { at wellhead; Seven Springs } \\
\text { Quadrangle. }\end{array}$ & \\
\hline 63 & $\begin{array}{l}\text { GRI Well at } \\
6300 \mathrm{ft}\end{array}$ & $V A-116$ & $1 / 83$ & $\begin{array}{l}\text { Lat } 35^{\circ} 54^{\prime} 34^{\prime \prime} \\
\text { Long } 106^{\circ} 37^{\prime} 53^{\prime \prime}\end{array}$ & $m$ & 232.6 & 7.10 & 90 & 30800 & -- & $\begin{array}{l}\text { Sample collected from downhole } \\
\text { sampling device; Seven Springs } \\
\text { Quadrangle }\end{array}$ & \\
\hline \multicolumn{13}{|c|}{ Valles Caldera - Ring Fracture Zone } \\
\hline 25 & Spence Hot Spring & VA-1 & $7 / 78$ & $\begin{array}{l}\text { Lat } 35^{\circ} 50^{\prime} 58^{\prime \prime} \\
\text { Long } 106^{\circ} 37^{\prime} 44^{\prime \prime}\end{array}$ & $\operatorname{tm}$ & 45 & 6.7 & -- & -- & 60 & $\begin{array}{l}\text { Spring issues from rhyolite } \\
\text { talus near contact with Abo } \\
\text { Formation; Jemez Springs } \\
\text { Quadrangle }\end{array}$ & $\begin{array}{l}\text { G-G; G-S; } \\
\text { P-W-A; S; } \\
\text { T74; T75 } \\
\text { T78 (H42) }\end{array}$ \\
\hline 25 & Spence Hot Spring & YA-6B & $6 / 81$ & $\begin{array}{l}\text { Lat } 35^{\circ} 50^{\prime} 58^{\prime \prime} \\
\text { Long } 106^{\circ} 37^{\prime} 44^{\prime \prime}\end{array}$ & tm & 42 & 7.01 & 320 & 280 & 80 & $\begin{array}{l}\text { Spring issues from rhyolite talus } \\
\text { near contact with Abo Formation; } \\
\text { Jemez Springs Quadrangle }\end{array}$ & \\
\hline 25 & Spence Hot Spring & VA-72 & $10 / 81$ & $\begin{array}{l}\text { Lat } 35^{\circ} 50^{\prime} 58^{\prime \prime} \\
\text { Long } 106^{\circ} 37^{\prime} 44^{\prime \prime}\end{array}$ & $\operatorname{tm}$ & 42 & 7.45 & 375 & 275 & 20 & $\begin{array}{l}\text { Spring issues from rhyolite talus } \\
\text { near contact with Abo Formation; } \\
\text { Jemez Springs Quadrangle }\end{array}$ & \\
\hline
\end{tabular}




\begin{tabular}{|c|c|c|c|c|c|c|c|c|c|c|c|c|}
\hline $\begin{array}{l}\text { Map } \\
\text { Ho. }\end{array}$ & Name & $\begin{array}{l}\text { Field } \\
\text { No. }\end{array}$ & Date & Location & $\begin{array}{l}\text { Water } \\
\text { Type }\end{array}$ & Temp & $\underset{\text { pH }}{\text { Field }}$ & $\begin{array}{l}\text { Field } \\
\text { Eh(my) }\end{array}$ & $\begin{array}{l}\text { Conduc. } \\
\text { (umhos/cm) }\end{array}$ & $\begin{array}{c}\text { Flow } \\
\text { Rate } \\
(\mathrm{e} / \mathrm{min})\end{array}$ & Comments & Reference $^{b}$ \\
\hline 25 & Spence Hot Spring & VA-83 & $3 / 82$ & $\begin{array}{l}\text { Lat } 35^{\circ} 50^{\prime} 58^{\prime \prime} \\
\text { Long } 106^{\circ} 37^{\prime} 44^{\prime \prime}\end{array}$ & $\operatorname{tm}$ & 41.6 & 7.87 & - & 275 & 160 & $\begin{array}{l}\text { Spring issues from rhyolite talus } \\
\text { near contact with Abo Forma tion; } \\
\text { Jemez Springs Quadrangle }\end{array}$ & \\
\hline 25 & Spence Hot Spring & $V A-105$ & $9 / 82$ & $\begin{array}{l}\text { Lat } 35^{\circ} 50^{\prime} 58^{\prime \prime} \\
\text { Long } 106^{\circ} 37^{\prime} 44^{\prime \prime}\end{array}$ & tm & 42.5 & - & -- & 315 & -- & $\begin{array}{l}\text { Spring issues from rhyolite talus } \\
\text { near contact with Abo Formation; } \\
\text { Jemez Springs Quadrangle }\end{array}$ & \\
\hline 25 & Spence Hot Spring & $V A-120$ & $1 / 83$ & $\begin{array}{l}\text { Lat } 35^{\circ} 50^{\prime} 58^{\prime \prime} \\
\text { Long } 106^{\circ} 37^{\prime} 44^{\prime \prime}\end{array}$ & $\operatorname{tm}$ & 42.3 & 7.60 & 28 & 293 & 160 & $\begin{array}{l}\text { Spring issues from rhyolite talus } \\
\text { near contact with Abo Formation; } \\
\text { Jemez Springs Quadrangle }\end{array}$ & \\
\hline 25 & $\begin{array}{l}\text { Little Spence } \\
\text { Hot Spring }\end{array}$ & $V A-2$ & $7 / 78$ & $\begin{array}{l}\text { Lat } 35^{\circ} 50^{\circ} 58^{\prime \prime} \\
\text { Long } 106^{\circ} 37^{\prime} 44^{\prime \prime}\end{array}$ & tm & 34 & 6.7 & -- & -- & 20 & $\begin{array}{l}\text { Spring issues from rhyolite talus } \\
\text { near contact with Abo Formation; } \\
\text { Jemez Springs Quadrangle }\end{array}$ & G-S \\
\hline 26 & McCauley Spring & VA-3 & $7 / 78$ & $\begin{array}{l}\text { Lat } 35^{\circ} 49^{\circ} 12^{\prime \prime} \\
\text { Long } 106^{\circ} 37^{\prime} 37^{\prime \prime}\end{array}$ & $\mathrm{tm}$ & 31 & 6.2 & -- & -- & 140 & $\begin{array}{l}\text { Spring flows from contact } \\
\text { of rhyolite and Abo Formation; } \\
\text { Jemez Springs Quadrangle }\end{array}$ & $\begin{array}{l}\text { G-S; P-W-A; } \\
T 78(H 39)\end{array}$ \\
\hline 26 & McCauley Spring & VA-87 & $3 / 82$ & $\begin{array}{l}\text { Lat } 35^{\circ} 49^{\prime} 12^{\prime \prime} \\
\text { Long } 106^{\circ} 37^{\prime} 37^{\prime \prime}\end{array}$ & $\mathrm{tm}$ & 31.5 & 7.87 & -- & 190 & $>400$ & $\begin{array}{l}\text { Spring flows from contact } \\
\text { of rhyolite and Abo Formation; } \\
\text { Jemez Springs Quadrangle }\end{array}$ & \\
\hline 26 & McCauley Spring & VA-119 & $1 / 83$ & $\begin{array}{l}\text { Lat } 35^{\circ} 49^{\prime} 12^{\prime \prime} \\
\text { Long } 106^{\circ} 37^{\prime} 37^{\prime \prime}\end{array}$ & $\operatorname{tm}$ & 31.9 & 8.23 & 49 & 173 & 960 & $\begin{array}{l}\text { Spring flows from contact } \\
\text { of rhyolite and Abo Formation; } \\
\text { Jemez Springs Quadrangle }\end{array}$ & \\
\hline 27 & $\begin{array}{l}\text { San Antonio } \\
\text { Hot Spring }\end{array}$ & $V A-4$ & $7 / 78$ & $\begin{array}{l}\text { Lat } 35^{\circ} 56^{\prime} 18^{\prime \prime} \\
\text { Long } 106^{\circ} 38^{\prime} 44^{\prime \prime}\end{array}$ & $\mathrm{tm}$ & 42 & 6.8 & -- & -- & 150 & $\begin{array}{l}\text { Spring discharges from concrete } \\
\text { crib on fractured rhyolite; } \\
\text { Seven Springs Quadrangle }\end{array}$ & $\begin{array}{l}\text { G-G; G-S; } \\
\text { P-W-A; S; } \\
\text { T75, T78 } \\
(P 12)\end{array}$ \\
\hline 27 & $\begin{array}{l}\text { San Antonio } \\
\text { Hot Spring }\end{array}$ & VA-96 & $3 / 82$ & $\begin{array}{l}\text { Lat } 35^{\circ} 56^{\prime} 18^{\prime \prime} \\
\text { Long } 106^{\circ} 38^{\prime} 44^{\prime \prime}\end{array}$ & tm & 40.8 & 7.26 & 374 & 140 & 125 & $\begin{array}{l}\text { Spring discharges from concrete } \\
\text { crib on fractured rhyolite; } \\
\text { Seven Springs Quadrangle }\end{array}$ & \\
\hline 27 & $\begin{array}{l}\text { San Antonio } \\
\text { Hot Spring }\end{array}$ & $V A-128$ & $3 / 83$ & $\begin{array}{l}\text { Lat } 35^{\circ} 56^{\prime} 18^{\prime \prime} \\
\text { Long } 106^{\circ} 38^{\prime} 44^{\prime \prime}\end{array}$ & $\mathrm{tm}$ & 41.3 & 7.88 & 84 & 127 & -- & $\begin{array}{l}\text { Spring discharges from concrete } \\
\text { crib on fractured rhyolite; } \\
\text { Seven Springs Quadrangle }\end{array}$ & \\
\hline 28 & Bathhouse Spring & $V A-20$ & $2 / 79$ & $\begin{array}{l}\text { Lat } 35^{\circ} 58^{\prime} 17^{\prime \prime} \\
\text { Long } 106^{\circ} 33^{\prime} 38^{\prime \prime}\end{array}$ & tm & 38 & 6.1 & -- & 163 & 12 & $\begin{array}{l}\text { Spring issues from south base of } \\
\text { small rhyoltte dome; covered by } \\
\text { wood bathhouse; Valie San Antonio } \\
\text { Quadrangle }\end{array}$ & 778 (P9) \\
\hline
\end{tabular}


TABLE B-I (cont)

\begin{tabular}{|c|c|c|c|c|c|c|c|c|c|c|c|c|}
\hline $\begin{array}{l}\text { Map } \\
\text { No. }\end{array}$ & Name & $\begin{array}{l}\text { Field } \\
\text { No. }\end{array}$ & Date & Location & $\begin{array}{l}\text { Hater } \\
\text { Type }\end{array}$ & $\stackrel{\text { Temp }}{\left({ }^{\circ} \mathrm{C}\right)}$ & $\begin{array}{l}\text { Field } \\
\text { pH }\end{array}$ & $\begin{array}{r}\text { Field } \\
\text { Eh(mV) }\end{array}$ & $\begin{array}{l}\text { Conduc. } \\
(\text { umhos } / \mathrm{cm})\end{array}$ & $\begin{array}{c}\text { Flow } \\
\text { Rate } \\
(\varepsilon / \mathrm{min})\end{array}$ & Comments & Reference $^{b}$ \\
\hline 28 & Bathhouse Spring & VA-94 & $3 / 82$ & $\begin{array}{l}\text { Lat } 35^{\circ} 58^{\prime} 17^{\prime \prime} \\
\text { Long } 106^{\circ} 33^{\prime} 38^{\prime \prime}\end{array}$ & $\operatorname{tm}$ & 37.4 & 7.72 & 386 & 178 & 18 & $\begin{array}{l}\text { Spring issues from south base of } \\
\text { small rhyolite dome; covered by } \\
\text { wood bathhouse; Valie San Antonio } \\
\text { Quadrangle }\end{array}$ & \\
\hline 28 & Bathhouse Spring & VA-126 & $3 / 83$ & $\begin{array}{l}\text { Lat } 35^{\circ} 58^{\prime} 17^{\prime \prime} \\
\text { Long } 106^{\circ} 33^{\prime} 38^{\prime \prime}\end{array}$ & $\operatorname{tm}$ & 38.1 & 7.61 & 28 & 166 & - & $\begin{array}{l}\text { Spring issues from south base of } \\
\text { small rhyolite dome; covered by } \\
\text { wood bathhouse; Valie San Antonio } \\
\text { Quadrangle }\end{array}$ & \\
\hline 34 & Battleship Seep & VA-31 & $8 / 79$ & $\begin{array}{l}\text { Lat } 35^{\circ} 49^{\prime} 46^{\prime \prime} \\
\text { Long } 106^{\circ} 38^{\prime} 42^{\prime \prime}\end{array}$ & $m$ & 19 & 8.37 & -- & 4200 & 1 & $\begin{array}{l}\text { Seep about } 1 / 4 \mathrm{~km} \text { WW of } \\
\text { Battleship Rock and just } \mathrm{H} \text { of } \\
\text { Hwy } 4 \text {; from Madera } 1 \text { imestone; } \\
\text { Jemez Springs Quadrangle }\end{array}$ & $\begin{array}{l}\text { P-P-0; T78 } \\
(K 5)\end{array}$ \\
\hline 34 & Battleship Seep & VA-50 & $4 / 80$ & $\begin{array}{l}\text { Lat } 35^{\circ} 49^{\circ} 46^{\prime \prime} \\
\text { Long } 106^{\circ} 38^{\prime} 42^{\prime \prime}\end{array}$ & m & 11 & 7.92 & 35 & 4200 & 1 & $\begin{array}{l}\text { Seep about } 1 / 4 \mathrm{~km} \text { WH of } \\
\text { Battleship Rock and just } W \text { of } \\
\text { Hwy 4; from Madera limestone; } \\
\text { Jemez Springs Quadrangle }\end{array}$ & \\
\hline 34 & Battleship Seep & VA-133 & $5 / 83$ & $\begin{array}{l}\text { Lat } 35^{\circ} 49^{\prime} 46^{\prime \prime} \\
\text { Long } 106^{\circ} 38^{\prime} 42^{\prime \prime}\end{array}$ & $m$ & 12 & 8.27 & $364^{c}$ & 4300 & -- & $\begin{array}{l}\text { Seep about } 1 / 4 \mathrm{~km} \text { WW of } \\
\text { Battleship Rock and just } W \text { of } \\
\text { Hwy } 4 \text {; from Madera limestone; } \\
\text { Jemez Springs Quadrangle }\end{array}$ & \\
\hline 34 & $\begin{array}{l}\text { East Fork } \\
\text { Jemez River }\end{array}$ & $V A-84$ & $3 / 82$ & $\begin{array}{l}\text { Lat } 35^{\circ} 49^{\prime} 46^{\prime \prime} \\
\text { Long } 106^{\circ} 38^{\prime} 42^{\prime \prime}\end{array}$ & $m$ & 4.4 & 8.06 & -- & 125 & $\gg>500$ & $\begin{array}{l}100 \mathrm{~m} \text { upstream of confluence } \\
\text { with San Antonio Creek at } \\
\text { Battleship Rock }\end{array}$ & \\
\hline 34 & $\begin{array}{l}\text { East Fork } \\
\text { Jemez River }\end{array}$ & VA-106 & $9 / 82$ & $\begin{array}{l}\text { Lat } 35^{\circ} 49^{\prime} 46^{\prime \prime} \\
\text { Long } 106^{\circ} 38^{\prime} 42^{\prime \prime}\end{array}$ & $m$ & -- & -- & - & -- & -- & $\begin{array}{l}100 \mathrm{~m} \text { upstream of confluence } \\
\text { with San Antonio Creek at } \\
\text { Battleship Rock }\end{array}$ & \\
\hline 39 & $\begin{array}{l}\text { Valle Grande } \\
\text { Spring }\end{array}$ & $V A-42$ & $10 / 79$ & $\begin{array}{l}\text { Lat } 35^{\circ} 51^{\circ} 30^{\prime \prime} \\
\text { Long } 106^{\circ} 27^{\prime} 00^{\prime \prime}\end{array}$ & $m$ & 15 & 7.20 & 160 & 88 & 30 & $\begin{array}{l}\text { Spring issues from rhyolite } \\
\text { colluvium; Bland Quadrangle }\end{array}$ & \\
\hline 39 & $\begin{array}{l}\text { Valle Grande } \\
\text { Spring }\end{array}$ & VA-82 & $3 / 82$ & $\begin{array}{l}\text { Lat } 35^{\circ} 51^{\prime} 30^{\prime \prime} \\
\text { Long } 106^{\circ} 27^{\prime} 00^{\prime \prime}\end{array}$ & $m$ & 13.6 & 7.36 & - & 82 & 30 & $\begin{array}{l}\text { Spring issues from rhyolite } \\
\text { colluvium; Bland Quadrangle }\end{array}$ & \\
\hline 39 & $\begin{array}{l}\text { Valle Grande } \\
\text { Spring }\end{array}$ & VA-117 & $1 / 83$ & $\begin{array}{l}\text { Lat } 35^{\circ} 52^{\prime} 30^{\prime \prime} \\
\text { Long } 106^{\circ} 27^{\prime} 00^{\prime \prime}\end{array}$ & $m$ & 13.8 & 6.90 & 408 & 76 & 30 & $\begin{array}{l}\text { Spring issues from rhyolite } \\
\text { colluvium; Bland Quadrangle }\end{array}$ & \\
\hline 42 & Horseshoe Spring & VA-45 & $10 / 79$ & $\begin{array}{l}\text { Lat } 35^{\circ} 52^{\prime} 41^{\prime \prime} \\
\text { Long } 106^{\circ} 39^{\prime} 23^{\prime \prime}\end{array}$ & $m$ & 12 & 6.89 & -- & 240 & 12 & $\begin{array}{l}\text { Spring issues from rhyolite } \\
\text { alluvium; Seven Springs } \\
\text { Quadrangle }\end{array}$ & $\begin{array}{l}P-W-A ; T 78 \\
(W 15)\end{array}$ \\
\hline
\end{tabular}




\begin{tabular}{|c|c|c|c|c|c|c|c|c|c|c|c|}
\hline $\begin{array}{l}\text { Map } \\
\text { No. }\end{array}$ & Name & $\begin{array}{l}\text { Field } \\
\text { No. }\end{array}$ & Date & Location & $\begin{array}{l}\text { Nater } \\
\text { Type }\end{array}$ & Temp & $\underset{\mathrm{pH}}{\text { Ffeld }}$ & $\begin{array}{c}\text { Field } \\
\text { Eh(mV) }\end{array}$ & $\begin{array}{l}\text { Conduc. } \\
\text { (umhos/cm) }\end{array}$ & $\begin{array}{l}\text { Flow } \\
\text { Rate } \\
(\ell / \min )\end{array}$ & Reference $^{\mathbf{b}}$ \\
\hline 42 & Horseshoe Spring & VA-118 & $1 / 83$ & $\begin{array}{l}\text { Lat } 35^{\circ} 52^{\prime} 41^{\prime \prime} \\
\text { Long } 106^{\circ} 39^{\prime} 23^{\prime \prime}\end{array}$ & $m$ & 11.3 & 7.44 & 73 & 279 & 25 & $\begin{array}{l}\text { Spring issues from rhyolite } \\
\text { alluvium; Seven Springs } \\
\text { Quadrangle }\end{array}$ \\
\hline 61 & PCl at $225 \mathrm{ft}$ & $\mathrm{PC1}-7$ & $10 / 83$ & $\begin{array}{l}\text { Lat } 35^{\circ} 52^{\prime} 33^{\prime \prime} \\
\text { Long } 106^{\circ} 39^{\prime} 42^{\prime \prime}\end{array}$ & d & -- & $8.39^{c}$ & $437^{C}$ & $225^{c}$ & 1 & $\begin{array}{l}\text { Well at } 225 \mathrm{ft} \text {; Seven Springs } \\
\text { Quadrangle }\end{array}$ \\
\hline 61 & PC1 at $365-391 \mathrm{ft}$ & PC1-8 & $10 / 83$ & $\begin{array}{l}\text { Lat } 35^{\circ} 52^{\prime} 33^{\prime \prime} \\
\text { Long } 106^{\circ} 39^{\prime} 42^{\prime \prime}\end{array}$ & d & $\cdots$ & $8.05^{c}$ & $479^{C}$ & $200^{c}$ & 0 & Hell at $365-391 \mathrm{ft}$; static water at $285 \mathrm{ft}$ \\
\hline 61 & $\mathrm{PCl}$ at $685-691 \mathrm{ft}$ & PC1-9 & $10 / 83$ & $\begin{array}{l}\text { Lat } 35^{\circ} 52^{\prime} 33^{\prime \prime} \\
\text { Long } 106^{\circ} 39^{\prime} 42^{\prime \prime}\end{array}$ & d & - & $8.38^{c}$ & $284^{C}$ & $1325^{C}$ & 0 & Hel1 at $685-691 \mathrm{ft}$ \\
\hline 61 & PC1 at $943 \mathrm{ft}$ & PC1-10 & $10 / 83$ & $\begin{array}{l}\text { Lat } 35^{\circ} 52^{\prime} 33^{\prime \prime} \\
\text { Long } 106^{\circ} 39^{\prime} 42^{\prime \prime}\end{array}$ & d & - & $8.80^{c}$ & $230^{c}$ & $1770^{C}$ & 0 & Well at $943 \mathrm{ft}$; mud sample \\
\hline 61 & $\mathrm{PCl}$ at $1100 \mathrm{ft}$ & $P C 1-11$ & $10 / 83$ & $\begin{array}{l}\text { Lat } 35^{\circ} 52^{\prime} 33^{\prime \prime} \\
\text { Long } 106^{\circ} 39^{\prime} 42^{\prime \prime}\end{array}$ & d & - & $7.55^{c}$ & $350^{c}$ & $3700^{c}$ & 0 & $\begin{array}{l}\text { Well at } 1100 \mathrm{ft} \text {; static level at } 960 \mathrm{ft} \text {; would } \\
\text { not bail }\end{array}$ \\
\hline 61 & PC1 at $1712 \mathrm{ft}$ & PC1-1 & $4 / 84$ & $\begin{array}{l}\text { Lat } 35^{\circ} 52^{\prime} 33^{\prime \prime} \\
\text { Long } 106^{\circ} 39^{\prime} 42^{\prime \prime}\end{array}$ & d & -- & 7.21 & 317 & 9500 & 0 & At $1712 \mathrm{~m}$ \\
\hline 61 & $\mathrm{PCl}$ at $1937 \mathrm{ft}$ & PC1-2 & $4 / 84$ & $\begin{array}{l}\text { Lat } 35^{\circ} 52^{\prime} 33^{\prime \prime} \\
\text { Long } 106^{\circ} 39^{\prime} 42^{\prime \prime}\end{array}$ & d & -- & 12.05 & 176 & 10800 & 0 & At $1937 \mathrm{~m}$ \\
\hline 61 & PCl at $1987 \mathrm{ft}$ & $\mathrm{PC} 1-3$ & $4 / 84$ & $\begin{array}{l}\text { Lat } 35^{\circ} 52^{\prime} 33^{\prime \prime} \\
\text { Long } 106^{\circ} 39^{\prime} 42^{\prime \prime}\end{array}$ & d & - & 12.17 & 125 & 14500 & 0 & At $1987 \mathrm{~m}$, top of cement \\
\hline 61 & $\mathrm{PCl}$ at $1953 \mathrm{ft}$ & $\mathrm{PCl}-4$ & $4 / 84$ & $\begin{array}{l}\text { Lat } 35^{\circ} 52^{\prime} 33^{\prime \prime} \\
\text { Long } 106^{\circ} 39^{\prime} 42^{\prime \prime}\end{array}$ & d & - & 7.63 & 347 & 11700 & 0 & At $1953 \mathrm{~m}$ \\
\hline 61 & PC1 at $2036 \mathrm{ft}$ & $\mathrm{PCl}-5$ & $5 / 84$ & $\begin{array}{l}\text { Lat } 35^{\circ} 52^{\prime} 33^{\prime \prime} \\
\text { Long } 106^{\circ} 39^{\prime} 42^{\prime \prime}\end{array}$ & d & -- & 8.05 & 327 & 9300 & 0 & At 2036 m \\
\hline 71 & $\mathrm{PC2}$ at $587-600 \mathrm{ft}$ & PC2-1 & $8 / 84$ & $\begin{array}{l}\text { Lat } 35^{\circ} 52^{\prime} 35^{\prime \prime} \\
\text { Long } 106^{\circ} 41^{\prime} 15^{\prime \prime}\end{array}$ & d & 30 & 8.55 & 307 & 1196 & -- & Total depth well $=1830 \mathrm{ft}$ \\
\hline 71 & PC2 at $630-635 \mathrm{ft}$ & PC2-2 & $8 / 84$ & $\begin{array}{l}\text { Lat } 35^{\circ} 52^{\prime} 35^{\prime \prime} \\
\text { Long } 106^{\circ} 41^{\prime} 15^{\prime \prime}\end{array}$ & d & 30 & 8.72 & 370 & 1232 & -- & Total depth well $=1830 \mathrm{ft}$ \\
\hline 71 & PC2 at $1086 \mathrm{ft}$ & $P C 2-3$ & $9 / 84$ & $\begin{array}{l}\text { Lat } 35^{\circ} 52^{\prime} 35^{\prime \prime} \\
\text { Long } 106^{\circ} 41^{\prime} 15^{\prime \prime}\end{array}$ & d & -- & 8.56 & 390 & 1260 & -- & $\begin{array}{l}\text { Lower Abo aquifer, sample is } \\
\text { mixture of mud and water }\end{array}$ \\
\hline 71 & PC2 at $1350 \mathrm{ft}$ & PC2-4 & $9 / 84$ & $\begin{array}{l}\text { Lat } 35^{\circ} 52^{\prime} 35^{\prime \prime} \\
\text { Long } 105^{\circ} 41^{\prime} 15^{\prime \prime}\end{array}$ & d & 35 & 7.18 & 410 & 2310 & - & Madera Formation \\
\hline
\end{tabular}


TABLE B-I (cont)

\begin{tabular}{|c|c|c|c|c|c|c|c|c|c|c|c|c|}
\hline $\begin{array}{l}\text { Map } \\
\text { No. }\end{array}$ & Name & $\begin{array}{l}\text { Field } \\
\text { No. }\end{array}$ & Date & Location & $\begin{array}{l}\text { Wa ter } \\
\text { Type }\end{array}$ & $\begin{array}{l}\text { Temp } \\
(\cdot \mathrm{c})\end{array}$ & $\begin{array}{l}\text { Field } \\
\text { pH }\end{array}$ & $\begin{array}{l}\text { Field } \\
\text { Eh(mV) }\end{array}$ & $\begin{array}{l}\text { Conduc. } \\
\text { (umhos/cm) }\end{array}$ & $\begin{array}{c}\text { Flow } \\
\text { Rate } \\
(2 / m i n)\end{array}$ & Comments & Reference $^{b}$ \\
\hline 71 & PC2 at $1360 \mathrm{ft}$ & PC2-5 & $9 / 84$ & $\begin{array}{l}\text { Lat } 35^{\circ} 52^{\prime} 35^{\prime \prime} \\
\text { Long } 106^{\circ} 41^{\prime} 15^{\prime \prime}\end{array}$ & d & 38 & 7.13 & 430 & 2370 & -- & Madera Formation & \\
\hline 71 & PC2 at $1335 \mathrm{ft}$ & PC2-6 & $9 / 84$ & $\begin{array}{l}\text { Lat } 35^{\circ} 52^{\prime} 35^{\prime \prime} \\
\text { Long } 106^{\circ} 41^{\prime} 15^{\prime \prime}\end{array}$ & d & 40 & 7.17 & 392 & 4980 & -- & Madera Formation & \\
\hline 71 & PC2 at $1490 \mathrm{ft}$ & PC2-7 & $10 / 84$ & $\begin{array}{l}\text { Lat } 35^{\circ} 52^{\prime} 35^{\prime \prime} \\
\text { Long } 106^{\circ} 41^{\prime} 15^{\prime \prime}\end{array}$ & $d$ & 41 & 7.13 & 443 & 2610 & -- & Total depth well $=1830 \mathrm{ft}$ & \\
\hline \multicolumn{13}{|c|}{ Valles Caldera - Baca Geothermal Field $^{d}$} \\
\hline 62 & $\begin{array}{l}\text { Redondo Creek } \\
\text { at Union Gate }\end{array}$ & VA-129. & $5 / 83$ & $\begin{array}{l}\text { Lat } 35^{\circ} 52^{\prime} 22^{\prime \prime} \\
\text { Long } 106^{\circ} 37^{\prime} 16^{\prime \prime}\end{array}$ & $m$ & 4 & 6.87 & 325 & 120 & -- & $\begin{array}{l}\text { At } 2440 \mathrm{~m} \text {; Valle San Antonio } \\
\text { Quadrangle }\end{array}$ & \\
\hline 64 & Baca Hell 4 & $B A-2$ & $6 / 82$ & $\begin{array}{l}\text { Lat } 35^{\circ} 53^{\prime} 21^{\prime \prime} \\
\text { Long } 106^{\circ} 34^{\prime} 13^{\prime \prime}\end{array}$ & d & 294 & 7.28 & $99^{c}$ & 9100 & -- & $\begin{array}{l}\text { Valle San Antgnio Quadrangle } \\
y^{5}=0.281, p^{\prime}=8.85 \text { bars ab. }\end{array}$ & \\
\hline 64 & Baca We11 4 & $B A-5$ & $7 / 82$ & $\begin{array}{l}\text { Lat } 35^{\circ} 53^{\prime} 21^{\prime \prime} \\
\text { Long } 106^{\circ} 34^{\prime} 13^{\prime \prime}\end{array}$ & d & 297 & 7.20 & - & 9100 & -- & $\begin{array}{l}\text { Valle San Antgnio Quadrangle } \\
y^{g}=0.287, p^{3}=9.23 \text { bars ab. }\end{array}$ & \\
\hline 65 & Baca Well 13 & $B A-1$ & $6 / 82$ & $\begin{array}{l}\text { Lat } 35^{\circ} 53^{\prime} 47^{\prime \prime} \\
\text { Long } 106^{\circ} 34^{\prime} 04^{\prime \prime}\end{array}$ & d & 278 & 7.30 & $75^{c}$ & 8500 & - & $\begin{array}{l}\text { Valle San Antonio Quadrangle } \\
y^{\xi}=0.238, p^{g}=9.05 \text { bars ab. }\end{array}$ & \\
\hline 65 & Baca Well \#13 & $B A-4$ & $7 / 82$ & $\begin{array}{l}\text { Lat } 35^{\circ} 53^{\prime} 47^{\prime \prime} \\
\text { Long } 106^{\circ} 34^{\prime} 04^{\prime \prime}\end{array}$ & d & 279 & 7.20 & $219^{c}$ & 8900 & -- & $\begin{array}{l}\text { Valle San Antgnio Quadrangle } \\
y^{5}=0.238, p^{\xi}=9.12 \text { bars ab. }\end{array}$ & \\
\hline 66 & Baca Well \#15 & $B A-7$ & $7 / 82$ & $\begin{array}{l}\text { Lat } 35^{\circ} 53^{\prime} 36^{\prime \prime} \\
\text { Long } 106^{\circ} 34^{\prime} 48^{\prime \prime}\end{array}$ & d & 267 & 7.61 & 3 & 10400 & -- & $\begin{array}{l}\text { Valle San Antgnio Quadrangle } \\
y^{5}=0.208, p^{5}=9.37 \text { bars ab. }\end{array}$ & \\
\hline 66 & Baca Hell 15 & $B A-8$ & $9 / 82$ & $\begin{array}{l}\text { Lat } 35^{\circ} 53^{\prime} 36^{\prime \prime} \\
\text { Long } 106^{\circ} 34^{\prime} 48^{\prime \prime}\end{array}$ & d & 326 & 7.12 & $107^{C}$ & 10600 & -- & $\begin{array}{l}\text { Valle San Antgnio Quadrangle } \\
y^{5}=0.359, p^{s}=9.37 \text { bars ab. }\end{array}$ & \\
\hline 66 & Baca Well 19 & $B A-9$ & $10 / 82$ & $\begin{array}{l}\text { Lat } 35^{\circ} 53^{\prime} 36^{\prime \prime} \\
\text { Long } 106^{\circ} 34^{\prime} 48^{\prime \prime}\end{array}$ & d & 223 & 8.45 & $213^{\mathrm{C}}$ & 10900 & -- & $\begin{array}{l}\text { Valle San Antgnio Quadrangle } \\
y^{5}=0.202, p^{5}=2.16 \text { bars ab. }\end{array}$ & \\
\hline 67 & Baca Well 24 & $B A-3$ & $6 / 82$ & $\begin{array}{l}\text { Lat } 35^{\circ} 53^{\prime} 04^{\prime \prime} \\
\text { Long } 106^{\circ} 34^{\prime} 57^{\prime \prime}\end{array}$ & $d$ & 260 & 7.25 & $245^{c}$ & 10600 & -- & $\begin{array}{l}\text { Valle San Antonio Quadrangle } \\
y^{5}=0.189, p^{5}=9.47 \text { bars ab. }\end{array}$ & \\
\hline 67 & Baca Hell \#24 & $B A-6$ & $7 / 82$ & $\begin{array}{l}\text { Lat } 35^{\circ} 53^{\circ} 04^{\prime \prime} \\
\text { Long } 106^{\circ} 34^{\prime} 57^{\prime \prime}\end{array}$ & d & 261 & 7.43 & $139^{C}$ & 10400 & -- & $\begin{array}{l}\text { Valle San Antonio Quadrangle } \\
y^{s}=0.193, p^{5}=9.12 \text { bars ab. }\end{array}$ & \\
\hline
\end{tabular}




\begin{tabular}{|c|c|c|c|c|c|c|c|c|c|c|c|c|}
\hline $\begin{array}{l}\text { Map } \\
\text { No. }\end{array}$ & Name & $\begin{array}{l}\text { Field } \\
\text { No. }\end{array}$ & Date & Location & $\begin{array}{l}\text { Wa ter } \\
\text { Type }\end{array}$ & $\underset{(\cdot \operatorname{comp}}{ }$ & $\underset{\mathrm{pH}}{\text { Field }}$ & $\begin{array}{r}\text { Field } \\
\text { Eh(mV) }\end{array}$ & $\begin{array}{l}\text { Conduc. } \\
(\text { mhos } / \mathrm{cm})\end{array}$ & $\begin{array}{c}\text { Flow } \\
\text { Rate } \\
(\ell / m i n)\end{array}$ & Comments & Reference ${ }^{b}$ \\
\hline
\end{tabular}

Valles Caldera - Soda Dam and Jemez Springs Area

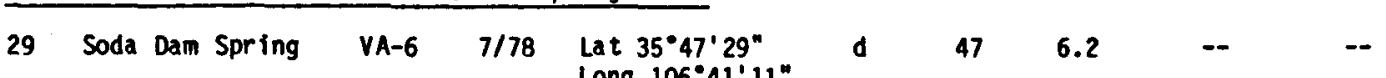

29

29 Soda Dam Spring

29 Soda Dam Spring

29 Soda Dam Spring

VA-73

VA-64

$12 / 80$

Lat $35^{\circ} 47^{\prime} 29^{\prime \prime}$
Long $106^{\circ} 41^{\prime} 11^{\prime \prime}$

VA-70 6/81 Lat $35^{\circ} 47^{\prime} 29^{\prime \prime}$ d

Lat $35^{\circ} 47^{\prime} 29^{\prime \prime}$
Long $106^{\circ} 41^{\prime} 11^{\prime \prime}$

\section{(a)}

Lat $35^{\circ} 47^{\prime} 29^{\prime \prime}$
Long $106^{\circ} 41^{\prime} 11^{\prime \prime}$

29 Soda Dam Spring

VA-89 $3 / 8$

Lat $35^{\circ} 47^{\prime} 29^{\prime \prime}$
Long $106^{\circ} 41^{\prime} 11^{\prime \prime}$

29 Soda Dam Spring

VA-99 $8 / 82$ Lat $35^{\circ} 47^{\prime} 29^{\prime \prime}$ spring discharges from faulted Paleozoic sediments in Jemez fault zone; $\mathrm{CO}_{2}^{4}, \mathrm{H}_{2} \mathrm{St}$;

60 Main spring discharges from faulted Paleozoic sediments in Jemez fault zone; $\mathrm{CO}_{2}+\mathrm{H}_{2} \mathrm{St}$; Jemez faur tone; $\mathrm{CO}_{2}{ }^{4}, \mathrm{H}_{2}$

60 Main spring discharges from faulted Paleozoic sediments in Jemez fault zone; $\mathrm{CO}_{2}, \mathrm{H}_{2} \mathrm{St}$; Jemez fauit zone, $\mathrm{CO}_{2}{ }_{3}, \mathrm{H}_{2} \mathrm{~S}$

Jemez Springs Quadrangle

faulted Paleozoic sediments in Jemez fault zone; $\mathrm{CO}_{2}{ }_{1}, \mathrm{H}_{2} \mathrm{St}$; Jemez fault zone; $\mathrm{CO}_{2}{ }^{4}, \mathrm{H}_{2} \mathrm{St}^{4}$
Jemez Springs Quadrangle

60 Main spring discharges from faulted paleozoic sediments in Jemez fault zone; $\mathrm{CO}_{2}^{+}, \mathrm{H}_{2} \mathrm{St}^{\text {; }}$ Jemez Springs Quadrangle

60 Main spring discharges from faulted Paleozoic sediments in Jemez fault zone; $\mathrm{CO}_{2}+\mathrm{H}_{2} \mathrm{St}$; Jemez Springs Quadrangle

40 Main spring discharges from faulted Paleozoic sediments in Jemez fault zone; $\mathrm{CO}_{2}{ }^{4}, \mathrm{H}_{2} \mathrm{SH}$; Jemez Springs Quadrangle

40 Main spring discharges from faulted Paleozoic sediments in Jemez fault zone; $\mathrm{CO}_{2}+, \mathrm{H}_{2} \mathrm{St}$; Jemez Springs Quadrangie

- Main spríng discharges from faulted Paleozoic sediments in Jemez fault zone; $\mathrm{CO}_{2}, \mathrm{H}_{2} \mathrm{St}^{+}$; Jemez fault zone; $\mathrm{CO}_{2}{ }^{4}, \mathrm{H}_{2} \mathrm{~S}+$
Jemez Springs Quadrangie
G-G; G et

a $7 ;$ G-S; P-W-A; S; T74; T75; T78 (H6) 
TABLE B-I (cont)

\begin{tabular}{|c|c|c|c|c|c|c|c|c|c|c|c|c|}
\hline $\begin{array}{l}\text { Map } \\
\text { No. }\end{array}$ & Name & $\begin{array}{l}\text { Field } \\
\text { No. }\end{array}$ & Date & Location & $\begin{array}{l}\text { Water } \\
\text { Type }\end{array}$ & $\underset{(\cdot c)}{\text { Temp }}$ & $\underset{\mathrm{pH}}{\text { Field }}$ & $\begin{array}{c}\text { Field } \\
\text { Eh(mV) }\end{array}$ & $\begin{array}{l}\text { Conduc. } \\
\text { (umhos/cm) }\end{array}$ & $\begin{array}{l}\text { Flow } \\
\text { Rate } \\
(2 / \mathrm{min})\end{array}$ & Comments & Reference $^{b}$ \\
\hline 29 & Soda Dam Spring & $V A-109$ & $1 / 83$ & $\begin{array}{l}\text { Lat } 35^{\circ} 47^{\prime} 29^{\prime \prime} \\
\text { Long } 106^{\circ} 41^{\prime} 11^{\prime \prime}\end{array}$ & d & 46.8 & 6.21 & -240 & 7090 & 60 & $\begin{array}{l}\text { Main spring discharges from } \\
\text { faul ted Paieozoic sediments in } \\
\text { Jemez fault zone; } \mathrm{CO}_{2}{ }^{+}, \mathrm{H}_{2} \mathrm{~S}_{4} \text {; } \\
\text { Jemez Springs Quadraligle }\end{array}$ & \\
\hline 29 & Soda Dam Spring & VA-132 & $5 / 83$ & $\begin{array}{l}\text { Lat } 35^{\circ} 47^{\prime} 29^{\prime \prime} \\
\text { Long } 106^{\circ} 41^{\prime} 11^{\prime \prime}\end{array}$ & $d$ & 47 & 7.19 & -375 & 6800 & -- & $\begin{array}{l}\text { Main spring discharges from } \\
\text { faulted Paleozoic sediment's in } \\
\text { Jemez fault zone; } \mathrm{CO}_{2} \mathrm{H}_{2} \mathrm{H}_{2} \mathrm{St}^{-} \\
\text {Jemez Springs Quadrangle }\end{array}$ & \\
\hline 29 & Soda Dam Spring & VA-140 & $2 / 84$ & $\begin{array}{l}\text { Lat } 35^{\circ} 47^{\prime} 29^{\prime \prime} \\
\text { Long } 106^{\circ} 41^{\prime} 11^{\prime \prime}\end{array}$ & d & 46.8 & 6.71 & -200 & 7300 & - & $\begin{array}{l}\text { Main spriing discharges from } \\
\text { faulted Paleozbic sediments in } \\
\text { Jemez fault zone; } \mathrm{CO}_{2}{ }^{4}, \mathrm{H}_{2} \mathrm{St}^{+} \\
\text {Jemez Springs Quadrangle }\end{array}$ & \\
\hline 29 & Soda Dam Spring & VA-146 & $4 / 84$ & $\begin{array}{l}\text { Lat } 35^{\circ} 47^{\prime} 29^{\prime \prime} \\
\text { Long } 106^{\circ} 41^{\prime} 11^{\prime \prime}\end{array}$ & d & -- & 6.95 & -- & 6700 & -- & $\begin{array}{l}\text { Main spring discharges from } \\
\text { faulted Paleozoic sediments in } \\
\text { Jemez fault zone; } \mathrm{CO}_{2}^{+}, \mathrm{H}_{2} \mathrm{St}^{+} \\
\text {Jemez Springs Quadrangle }\end{array}$ & \\
\hline 29 & Grotto Spring & $V A-5$ & $7 / 78$ & $\begin{array}{l}\text { Lat } 35^{\circ} 47^{\prime} 29^{\prime \prime} \\
\text { Long } 106^{\circ} 41^{\prime} 11^{\prime \prime}\end{array}$ & d & 38 & 6.8 & -280 & - & 12 & $\begin{array}{l}\text { Spring flows from cave on } \\
\text { east side of Soda Dam; Jemez } \\
\text { Springs Quadrangle }\end{array}$ & G-S \\
\hline 29 & $\begin{array}{l}\text { Outfall of } \\
\text { Soda Dam Spring }\end{array}$ & $V A-65$ & $12 / 80$ & $\begin{array}{l}\text { Lat } 35^{\circ} 47^{\prime} 29^{\prime \prime} \\
\text { Long } 106^{\circ} 41^{\prime} 11^{\prime \prime}\end{array}$ & d & 17 & 8.13 & - & 6000 & 60 & $\begin{array}{l}\text { Sampled at point where water of } \\
\text { Soda Dam Spring enters Jemez River; } \\
\text { Jemez Springs Quadrangle }\end{array}$ & \\
\hline 29 & Hidden Warm Spring & $V A-27$ & $5 / 79$ & $\begin{array}{l}\text { Lat } 35^{\circ} 47^{\prime} 29^{\prime \prime} \\
\text { Long } 106^{\circ} 41^{\prime} 11^{\prime \prime}\end{array}$ & d & 29 & 6.28 & -280 & 5700 & 2 & $\begin{array}{l}\text { Spring issues from alluvium east } \\
\text { side Jemez River; Jemez Springs } \\
\text { Quadrangle }\end{array}$ & \\
\hline 29 & Hidden Warm Spring & VA-90 & $3 / 82$ & $\begin{array}{l}\text { Lat } 35^{\circ} 47^{\prime} 29^{\prime \prime} \\
\text { Long } 106^{\circ} 41^{\prime} 11^{\prime \prime}\end{array}$ & d & 32 & 6.2 & -- & 6000 & 6 & $\begin{array}{l}\text { Spring issues from alluvium east } \\
\text { side Jemez River; Jemez Springs } \\
\text { Quadrangle }\end{array}$ & \\
\hline 29 & Hidden Warm Spring & VA-110 & $1 / 83$ & $\begin{array}{l}\text { Lat } 35^{\circ} 47^{\prime} 29^{\prime \prime} \\
\text { Long } 106^{\circ} 41^{\prime} 11^{\prime \prime}\end{array}$ & d & 32.3 & 6.13 & -180 & 6150 & 8 & $\begin{array}{l}\text { Spring issues from alluvium east } \\
\text { side Jemez River; Jemez Springs } \\
\text { Quadrangle }\end{array}$ & \\
\hline 29 & Hidden Warm Spring & $V A-141$ & $2 / 84$ & $\begin{array}{l}\text { Lat } 35^{\circ} 47^{\prime} 29^{\prime \prime} \\
\text { Long } 106^{\circ} 41^{\prime} 11^{\prime \prime}\end{array}$ & d & 32.2 & 6.42 & -130 & 6260 & -- & $\begin{array}{l}\text { Spring issues from alluvium east } \\
\text { side Jemez River; Jemez Springs } \\
\text { Quadrangle }\end{array}$ & \\
\hline 30 & Main Jemez Spring & $V A-10$ & $1 / 79$ & $\begin{array}{l}\text { Lat } 35^{\circ} 46^{\prime} 24^{\prime \prime} \\
\text { Long } 106^{\circ} 41^{\prime} 20^{\prime \prime}\end{array}$ & $d$ & 55 & 7.01 & -- & 4200 & 20 & $\begin{array}{l}\text { Spring discharges from concrete } \\
\text { tank under gazebo; } \mathrm{CO}_{2}^{+} ; \text {Jeme } z \\
\text { Springs Quadrangle }\end{array}$ & $\begin{array}{l}\text { G-G; G et } \\
\text { al; T75; T78 } \\
(\text { His) }\end{array}$ \\
\hline
\end{tabular}




\begin{tabular}{|c|c|c|c|c|c|c|c|c|c|c|c|c|c|}
\hline $\begin{array}{l}\text { Map } \\
\text { No. }\end{array}$ & & Name & $\begin{array}{l}\text { Field } \\
\text { No. }\end{array}$ & Date & Location & $\begin{array}{l}\text { Wateg } \\
\text { Type }\end{array}$ & Temp & $\underset{\mathrm{pH}}{\text { Field }}$ & $\begin{array}{l}\text { Field } \\
\text { Eh(mV) }\end{array}$ & $\begin{array}{l}\text { Conduc. } \\
(\text { whhos } / \mathrm{cm})\end{array}$ & $\begin{array}{c}\text { Flow } \\
\text { Rate } \\
(2 / \mathrm{min})\end{array}$ & Comments & Reference $^{b}$ \\
\hline 30 & Main & Jemez Spring & VA-18 & $1 / 79$ & $\begin{array}{l}\text { Lat } 35^{\circ} 46^{\prime} 24^{\prime \prime} \\
\text { Long } 106^{\circ} 41^{\prime} 20^{\prime \prime}\end{array}$ & d & 36 & 7.51 & -- & 4250 & 0 & $\begin{array}{l}\text { Spring discharges from concrete } \\
\text { tank under gazebo; } \mathrm{CO}_{2}+\text {; Jeme } z \\
\text { Springs Quadrangle }\end{array}$ & G et al. \\
\hline 30 & Main & Jemez Spring & VA-93 & $3 / 82$ & $\begin{array}{l}\text { Lat } 35^{\circ} 46^{\prime} 24^{\prime \prime} \\
\text { Long } 106^{\circ} 41^{\prime} 20^{\prime \prime}\end{array}$ & $d$ & 46.3 & 6.66 & -- & 4270 & 20 & $\begin{array}{l}\text { Spring discharges from concre te } \\
\text { tank under gazebo; } \mathrm{CO}_{2}+; \text { Jemez } \\
\text { Springs Quadrangle }\end{array}$ & \\
\hline 30 & Main $J$ & Jemez Spring & VA-122 & $1 / 83$ & $\begin{array}{l}\text { Lat } 35^{\circ} 46^{\prime} 24^{\prime \prime} \\
\text { Long } 106^{\circ} 41^{\prime} 20^{\prime \prime}\end{array}$ & $d$ & 74.9 & 6.57 & -230 & 4380 & 3 & $\begin{array}{l}\text { Spring discharges from concre te } \\
\text { tank under gazebo; } \mathrm{CO}_{2}^{+} ; \text {Jemez } \\
\text { Springs Quadrangle }\end{array}$ & \\
\hline 30 & Main $J$ & Jemez Spring & $V A-143$ & $2 / 84$ & $\begin{array}{l}\text { Lat } 35^{\circ} 46^{\circ} 24^{\prime \prime} \\
\text { Long } 106^{\circ} 41^{\circ} 20^{\prime \prime}\end{array}$ & $d$ & 74.7 & 6.50 & -240 & 4460 & - & $\begin{array}{l}\text { Spring discharges from concrete } \\
\text { tank under gazebo; } \mathrm{CO}_{2}^{+;} \text {Jemez } \\
\text { Springs Quadrangle }\end{array}$ & \\
\hline 30 & Main $J$ & Jemez Spring & VA-147 & $4 / 84$ & $\begin{array}{l}\text { Lat } 35^{\circ} 46^{\prime} 24^{\prime \prime} \\
\text { Long } 106^{\circ} 41^{\prime} 20^{\prime \prime}\end{array}$ & $d$ & -- & 7.09 & -- & 3900 & -- & $\begin{array}{l}\text { Spring discharges from concre te } \\
\text { tank under gazebo; } \mathrm{CO}_{2}+\text {; Jeme } \mathrm{Z} \\
\text { Springs Quadrangle }\end{array}$ & \\
\hline 30 & $\begin{array}{l}\text { Jemez } \\
\text { Geothe }\end{array}$ & $\begin{array}{l}\text { Springs } \\
\text { lermal Well }\end{array}$ & $V A-121$ & $1 / 83$ & $\begin{array}{l}\text { Lat } 35^{\circ} 46^{\prime} 24^{\prime \prime} \\
\text { Long } 106^{\circ} 41^{\prime} 20^{\prime \prime}\end{array}$ & m & 73.3 & 6.50 & -232 & 4280 & 64 & $\begin{array}{l}\text { Well drilled north of main Jemez } \\
\text { Spring; Jemez Springs Quadrangle }\end{array}$ & \\
\hline 30 & $\begin{array}{l}\text { Jemez } \\
\text { Geothe }\end{array}$ & $\begin{array}{l}\text { Springs } \\
\text { lermal Well }\end{array}$ & $V A-144$ & $2 / 84$ & $\begin{array}{l}\text { Lat } 35^{\circ} 46^{\prime} 24^{\prime \prime} \\
\text { Long } 106^{\circ} 41^{\circ} 20^{\prime \prime}\end{array}$ & $m$ & 72.2 & 6.40 & -90 & 4670 & -- & $\begin{array}{l}\text { Well drilled north of main Jemez } \\
\text { Spring }\end{array}$ & \\
\hline 30 & Jemez & $\mathrm{We} I 1 / 24 \mathrm{~m}$ & $V A-19$ & $1 / 79$ & $\begin{array}{l}\text { Lat } 35^{\circ} 46^{\prime} 24^{\prime \prime} \\
\text { Long } 106^{\circ} 41^{\prime} 20^{\prime \prime}\end{array}$ & d & 68 & 6.64 & -- & 3300 & 120 & $\begin{array}{l}\text { Well drilled north of main } \\
\text { Jemez Spring; aquifer tapped at } \\
24 \mathrm{~m}\end{array}$ & G et al. \\
\hline 30 & Jemez & $\mathrm{We} 11 / 24 \mathrm{~m}$ & $V A-25$ & $5 / 79$ & $\begin{array}{l}\text { Lat } 35^{\circ} 46^{\prime} 24^{\prime \prime} \\
\text { Long } 106^{\circ} 41^{\circ} 20^{\prime \prime}\end{array}$ & d & 73.3 & 6.55 & -- & 3500 & 8 & $\begin{array}{l}\text { Well drilled north of main } \\
\text { Jemez Spring; aquifer tapped } \\
\text { at } 24 \mathrm{~m}\end{array}$ & \\
\hline 30 & Jemez & We11/152 m & $V A-15$ & $1 / 79$ & $\begin{array}{l}\text { Lat } 35^{\circ} 46^{\prime} 24^{\prime \prime} \\
\text { Long } 106^{\circ} 41^{\prime} 20^{\prime \prime}\end{array}$ & d & 60.5 & 6.69 & -- & 1700 & 80 & $\begin{array}{l}\text { Well drilied north of main } \\
\text { Jemez Spring; aquifer tapped } \\
\text { at } 152 \mathrm{~m}\end{array}$ & G et al. \\
\hline 30 & Jemez & Well/152 m & $Y A-21$ & $2 / 79$ & $\begin{array}{l}\text { Lat } 35^{\circ} 46^{\prime} 24^{\prime \prime} \\
\text { Long } 106^{\circ} 41^{\prime} 20^{\prime \prime}\end{array}$ & $d$ & 61 & 6.55 & - & 1830 & 20 & $\begin{array}{l}\text { Well drilled north of main } \\
\text { Jemez Spring; aquifer tapped } \\
\text { at } 152 \mathrm{~m}\end{array}$ & \\
\hline 30 & $\begin{array}{l}\text { Traver } \\
\text { Spring }\end{array}$ & rtine Mound & $V A-7$ & $1 / 79$ & $\begin{array}{l}\text { Lat } 35^{\circ} 46^{\prime} 24^{\prime \prime} \\
\text { Long } 106^{\circ} 41^{\prime} 20^{\prime \prime}\end{array}$ & d & 70 & 6.28 & -- & 4200 & 4 & $\begin{array}{l}\text { Spring issues from travertine } \\
\text { mound west of bathhouse; } \mathrm{CO}_{2}+; \\
\text { Jemez Springs Quadrangle }\end{array}$ & $\begin{array}{l}\text { Get al ; } \\
\text { T74; T78 } \\
(H 14)\end{array}$ \\
\hline
\end{tabular}


TABLE B-I (cont)

\begin{tabular}{|c|c|c|c|c|c|c|c|c|c|c|c|c|}
\hline $\begin{array}{l}\text { Map } \\
\text { No. }\end{array}$ & Name & $\begin{array}{l}\text { Field } \\
\text { No. }\end{array}$ & Date & Location & $\begin{array}{l}\text { Water } \\
\text { Type }\end{array}$ & $\begin{array}{l}\text { Temp } \\
\left({ }^{\circ} \mathrm{C}\right)\end{array}$ & $\begin{array}{l}\text { Field } \\
\text { pH }\end{array}$ & $\begin{array}{r}\text { Field } \\
\text { Eh }(m V)\end{array}$ & $\begin{array}{l}\text { Conduc. } \\
\text { (panhos/cm) }\end{array}$ & $\begin{array}{c}\text { Flow } \\
\text { Rate } \\
(2 / \mathrm{min})\end{array}$ & Comments & Reference $^{b}$ \\
\hline 30 & $\begin{array}{l}\text { Travertine Mound } \\
\text { Spring }\end{array}$ & YA-17 & $1 / 79$ & $\begin{array}{l}\text { Lat } 35^{\circ} 46^{\prime} 24^{\prime \prime} \\
\text { Long } 106^{\circ} 41^{\prime} 20^{\prime \prime}\end{array}$ & d & 72 & 6.66 & -- & 4100 & 4 & $\begin{array}{l}\text { Spring issues from travertine } \\
\text { mound west of bathhouse; } \mathrm{CO}_{2}{ }^{4}\end{array}$ & G et al. \\
\hline 30 & $\begin{array}{l}\text { Travertine Mound } \\
\text { Spring }\end{array}$ & VA-66 & $12 / 80$ & $\begin{array}{l}\text { Lat } 35^{\circ} 46^{\circ} 24^{\prime \prime} \\
\text { Long } 106^{\circ} 41^{\prime} 20^{\prime \prime}\end{array}$ & d & 72 & 6.66 & -205 & 3400 & 4 & $\begin{array}{l}\text { Spring issues from travertine } \\
\text { mound west of bathhouse; } \mathrm{CO}_{2}^{4}\end{array}$ & $G$ et al. \\
\hline 30 & $\begin{array}{l}\text { Travertine Mound } \\
\text { Spring }\end{array}$ & VA-71 & $6 / 81$ & $\begin{array}{l}\text { Lat } 35^{\circ} 46^{\prime} 24^{\prime \prime} \\
\text { Long } 106^{\circ} 41^{\prime} 20^{\prime \prime}\end{array}$ & $d$ & 72 & 6.12 & -314 & 3900 & 4 & $\begin{array}{l}\text { Spring issues from travertine } \\
\text { mound west of ba thhouse: } \mathrm{CO}_{2}{ }^{4}\end{array}$ & \\
\hline 30 & $\begin{array}{l}\text { Travertine Mound } \\
\text { Spring }\end{array}$ & $V A-91$ & $3 / 82$ & $\begin{array}{l}\text { Lat } 35^{\circ} 46^{\circ} 24^{\prime \prime} \\
\text { Long } 106^{\circ} 41^{\prime} 20^{\prime \prime}\end{array}$ & d & 72.3 & 6.47 & -- & 4540 & 4 & $\begin{array}{l}\text { Spring issues from travertine } \\
\text { mound we st of bathhouse: } \mathrm{CO}_{2}^{4}\end{array}$ & \\
\hline 30 & $\begin{array}{l}\text { Travertine Mound } \\
\text { Spring }\end{array}$ & VA-123 & $1 / 83$ & $\begin{array}{l}\text { Lat } 35^{\circ} 46^{\circ} 24^{\prime \prime} \\
\text { Long } 106^{\circ} 41^{\prime} 20^{\prime \prime}\end{array}$ & $d$ & 72.6 & 6.59 & -270 & 4360 & 3 & $\begin{array}{l}\text { Spring issues from travertine } \\
\text { mound west of bathhouse; } \mathrm{CO}_{2}^{4}\end{array}$ & \\
\hline 30 & $\begin{array}{l}\text { Travertine Mound } \\
\text { Spring }\end{array}$ & YA-142 & $2 / 84$ & $\begin{array}{l}\text { Lat } 35^{\circ} 46^{\prime} 24^{\prime \prime} \\
\text { Long } 106^{\circ} 41^{\prime} 20^{\prime \prime}\end{array}$ & d & 72.9 & 6.20 & -280 & 4740 & -- & $\begin{array}{l}\text { Spring issues from travertine } \\
\text { mound west of bathhouse; } \mathrm{CO}_{2}^{4}\end{array}$ & \\
\hline 30 & Buddhist Spring & VA-8 & $1 / 79$ & $\begin{array}{l}\text { Lat } 35^{\circ} 46^{\prime} 24^{\prime \prime} \\
\text { Long } 106^{\circ} 41^{\prime} 20^{\prime \prime}\end{array}$ & d & 49 & 6.38 & -- & 3300 & 4 & $\begin{array}{l}\text { Spring flows from manmade } \\
\text { pool by Jemez River: } \mathrm{CO}^{+} \text {; } \\
\text { Jemez Springs Quadrangle }\end{array}$ & Get al. \\
\hline 30 & Buddhist Spring & $V A-16$ & $1 / 79$ & $\begin{array}{l}\text { Lat } 35^{\circ} 46^{\prime} 24^{\prime \prime} \\
\text { Long } 106^{\circ} 41^{\prime} 20^{\prime \prime}\end{array}$ & d & 50 & 6.59 & -160 & 3300 & 4 & $\begin{array}{l}\text { Spring flows from mamade } \\
\text { pool by Jemez River; } \mathrm{CO}_{2}^{4}\end{array}$ & \\
\hline 30 & Buddhist Spring & VA-92 & $3 / 82$ & $\begin{array}{l}\text { Lat } 35^{\circ} 46^{\prime} 24^{\prime \prime} \\
\text { Long } 106^{\circ} 41^{\prime} 20^{\prime \prime}\end{array}$ & d & 43.2 & 6.49 & -- & 3200 & 15 & $\begin{array}{l}\text { Spring flows from manmade } \\
\text { pool by Jemez River; } \mathrm{CO}_{2}{ }^{4}\end{array}$ & \\
\hline 30 & Unnamed Spring & VA-12 & $1 / 79$ & $\begin{array}{l}\text { Lat } 35^{\circ} 46^{\prime} 24^{\prime \prime} \\
\text { Long } 106^{\circ} 41^{\prime} 20^{\prime \prime}\end{array}$ & d & 49 & 6.35 & -- & 4100 & 4 & $\begin{array}{l}\text { Spring discharges from marsh } 15 \mathrm{~m} \\
\mathrm{WH} \text { of main Jemez Spring (now } \\
\text { destroyed); } \mathrm{CO}_{2}+\text {; Jemez Springs } \\
\text { Quadrangle }\end{array}$ & \\
\hline \multicolumn{13}{|c|}{ San Ysidro - Jemez Pueblo Area } \\
\hline 36 & Ponderosa Spring & VA-39 & $3 / 81$ & $\begin{array}{l}\text { Lat } 35^{\circ} 37^{\prime} 47^{\prime \prime} \\
\text { Long } 106^{\circ} 42^{\prime} 29^{\prime \prime}\end{array}$ & c & 11 & 6.91 & -- & 2500 & seep & $\begin{array}{l}\text { Spring discharges from } \\
\text { Chinle Formation near } \\
\text { contact W/ overlying Zia } \\
\text { SS; tastes salty; Ponderosa } \\
\text { Quadrangle }\end{array}$ & T78 (E1) \\
\hline 36 & Ponderosa Spring & VA-135 & $5 / 83$ & $\begin{array}{l}\text { Lat } 35^{\circ} 37^{\circ} 47^{\prime \prime} \\
\text { Long } 106^{\circ} 42^{\prime} 29^{\prime \prime}\end{array}$ & c & 15 & 6.90 & 19 & 2600 & 3 & $\begin{array}{l}\text { Spring discharges from } \\
\text { Chinle formation near } \\
\text { contact w/ overlying zia } \\
\text { SS; tastes salty; Ponderosa } \\
\text { Quadrangle }\end{array}$ & \\
\hline
\end{tabular}




\begin{tabular}{|c|c|c|c|c|c|c|c|c|c|c|c|c|}
\hline $\begin{array}{l}\text { Map } \\
\text { No. }\end{array}$ & Name & $\begin{array}{l}\text { Field } \\
\text { No. }\end{array}$ & Da te & Location & $\begin{array}{l}\text { Wa ter } \\
\text { Type }\end{array}$ & $\begin{array}{l}\text { Temp } \\
\left({ }^{\circ} \mathrm{C}\right)\end{array}$ & $\begin{array}{c}\text { Field } \\
\mathrm{pH}\end{array}$ & $\begin{array}{l}\text { Field } \\
\text { Eh(mV) }\end{array}$ & $\begin{array}{l}\text { Conduc. } \\
\text { (umhos } / \mathrm{cm} \text { ) }\end{array}$ & $\begin{array}{l}\text { Flow } \\
\text { Rate } \\
(e / m i n)\end{array}$ & Comments & Reference $^{b}$ \\
\hline 37 & Cañon Spring & $V A-40$ & $4 / 81$ & $\begin{array}{l}\text { Lat } 35^{\circ} 40^{\prime} 20^{\prime \prime} \\
\text { Long } 106^{\circ} 45^{\prime} 31^{\prime \prime}\end{array}$ & $m$ & 18 & 7.58 & -- & 1090 & 1 & $\begin{array}{l}\text { Spring issues from fault } \\
\text { in Precambrian granite; Gilman } \\
\text { Quadrangle }\end{array}$ & T78 (D6) \\
\hline 55 & $\begin{array}{l}\text { San Ysidro } \\
\text { Warm Spring }\end{array}$ & VA-33 & $8 / 79$ & $\begin{array}{l}\text { Lat } 35^{\circ} 32^{\prime} 52^{\prime \prime} \\
\text { Long } 106^{\circ} 49^{\prime} 32^{\prime \prime}\end{array}$ & c & 27 & 6.57 & 80 & 11550 & 1 & $\begin{array}{l}\text { Seep from travertine mound } \\
\text { N of Hwy } 44 \text {; Chinle Fm.; San } \\
\text { Ysidro Quadrangle }\end{array}$ & $\begin{array}{l}\text { T74; } \\
\text { T78 (A1); M }\end{array}$ \\
\hline 55 & $\begin{array}{l}\text { San Ysidro } \\
\text { Warm Spring }\end{array}$ & $V A-130$ & $5 / 83$ & $\begin{array}{l}\text { Lat } 35^{\circ} 32^{\prime} 52^{\prime \prime} \\
\text { Long } 106^{\circ} 49^{\prime} 32^{\prime \prime}\end{array}$ & c & 22 & 6.97 & $354^{c}$ & 9400 & -- & $\begin{array}{l}\text { Seep from travertine mound } \\
N \text { of Hwy } 44 ; \text { Chinle Fm.; San } \\
\text { Ysidro Quadrangle }\end{array}$ & \\
\hline 55 & $\begin{array}{l}\text { San Ysidro } \\
\text { Warm Spring }\end{array}$ & $V A-148$ & $4 / 84$ & $\begin{array}{l}\text { Lat } 35^{\circ} 32^{\prime} 52^{\prime \prime} \\
\text { Long } 106^{\circ} 49^{\prime} 32^{\prime \prime}\end{array}$ & c & -- & 6.5 & -- & 10000 & -- & $\begin{array}{l}\text { Seep from travertine mound } \\
N \text { of Hwy } 44 \text {; Chinle Fm.; San } \\
\text { Ysidro Quadrangle }\end{array}$ & \\
\hline 56 & Zia Hot Well & $V A-34$ & $8 / 79$ & $\begin{array}{l}\text { Lat } 35^{\circ} 38^{\prime} 44^{\prime \prime} \\
\text { Long } 106^{\circ} 53^{\prime} 19^{\prime \prime}\end{array}$ & c & 56 & 6.29 & -- & 16600 & 150 & $\begin{array}{l}\text { Artesian well flows from } \\
\text { concrete crib E of Hwy } 44 ; \\
\text { Chinle } \mathrm{Fm} . ; \mathrm{CO}_{2}+\text {; Holy Ghost } \\
\text { Spring Quadrangle }\end{array}$ & $\begin{array}{l}\mathrm{T} 74 ; \mathrm{T} 78 \\
(\mathrm{C} 3)\end{array}$ \\
\hline 56 & Zia Hot Well & $V A-53$ & $4 / 80$ & $\begin{array}{l}\text { Lat } 35^{\circ} 38^{\prime} 44^{\prime \prime} \\
\text { Long } 106^{\circ} 53^{\prime} 19^{\prime \prime}\end{array}$ & c & 54 & 6.53 & -150 & 16000 & 150 & $\begin{array}{l}\text { Artesian well flows from } \\
\text { concrete crib E of Hwy 44; } \\
\text { Chinle Fm.; } \mathrm{CO}_{2}+\text {; Holy Ghost } \\
\text { Spring Quadrangle }\end{array}$ & \\
\hline 56 & Zia Hot Well & $V A-67$ & $3 / 81$ & $\begin{array}{l}\text { Lat } 35^{\circ} 38^{\prime} 44^{\prime \prime} \\
\text { Long } 106^{\circ} 53^{\prime} 19^{\prime \prime}\end{array}$ & c & 53 & 6.72 & - & 15800 & 150 & $\begin{array}{l}\text { Artesian well flows from } \\
\text { concrete crib E of Hwy } 44 \text {; } \\
\text { Chinle Fm.; } \mathrm{CO}_{2} 4 \text {; Holy Ghost } \\
\text { Spring Quadrangle }\end{array}$ & \\
\hline 56 & Zia Hot Well & VA -74 & $10 / 81$ & $\begin{array}{l}\text { Lat } 35^{\circ} 37^{\prime} 44^{\prime \prime} \\
\text { Long } 106^{\circ} 53^{\prime} 19^{\prime \prime}\end{array}$ & c & 53 & 6.40 & 90 & 15600 & 240 & $\begin{array}{l}\text { Artesian well flows from } \\
\text { concrete crib E of Hwy } 44 \text {; } \\
\text { Chinle } \mathrm{Fm}^{\circ} \text {; } \mathrm{CO}_{2}+\text {; Holy Ghost } \\
\text { Spring Quadrangle }\end{array}$ & \\
\hline 56 & Zia Hot Well & $V A-125$ & $2 / 83$ & $\begin{array}{l}\text { Lat } 35^{\circ} 38^{\prime} 44^{\prime \prime} \\
\text { Long } 106^{\circ} 53^{\prime} 19^{\prime \prime}\end{array}$ & c & 53 & 6.98 & -50 & 15700 & 320 & $\begin{array}{l}\text { Artesian well flows from } \\
\text { concrete crib E of Hwy } 44 \text {; } \\
\text { Chinle Fm.; } \mathrm{CO}_{2}^{+} ; \text {Holy Ghost } \\
\text { Spring Quadrangle }\end{array}$ & \\
\hline 56 & Zia Hot Well & $V A-149$ & $4 / 84$ & $\begin{array}{l}\text { Lat } 35^{\circ} 38^{\prime} 44^{\prime \prime} \\
\text { Long } 106^{\circ} 53^{\prime} 19^{\prime \prime}\end{array}$ & $c$ & -- & 6.81 & -- & 15500 & -- & $\begin{array}{l}\text { Artesian well flows from } \\
\text { concrete crib E of Hwy } 44 \text {; } \\
\text { Chinle Fm.: } \mathrm{CO}_{2}^{+;} \text {Holy Ghost } \\
\text { Spring Quadrangle }\end{array}$ & \\
\hline
\end{tabular}


TABLE B-I (cont)

\begin{tabular}{|c|c|c|c|c|c|c|c|c|c|c|c|c|}
\hline $\begin{array}{l}\text { Map } \\
\text { Ho. }\end{array}$ & Name & $\begin{array}{l}\text { Field } \\
\text { No. }\end{array}$ & Date & Location & $\begin{array}{l}\text { Hater } \\
\text { Type }\end{array}$ & Temp & $\underset{\text { pH }}{\text { Field }}$ & $\begin{array}{l}\text { Field } \\
\text { Eh(mV) }\end{array}$ & $\begin{array}{l}\text { Conduc. } \\
\text { (umhos } / \mathrm{cm} \text { ) }\end{array}$ & $\begin{array}{c}\text { Flow } \\
\text { Rate } \\
(e / \text { min })\end{array}$ & Comments & Reference $^{b}$ \\
\hline 57 & Unnamed Hell & VA-35 & $8 / 79$ & $\begin{array}{l}\text { Lat } 35^{\circ} 42^{\prime} 32^{\prime \prime} \\
\text { Long } 105^{\circ} 59^{\prime} 42^{\prime \prime}\end{array}$ & m & 21 & -- & -- & -- & $1 / 2$ & $\begin{array}{l}\text { Well drains into cattle trough } \\
\text { above San Luis Tank; Morrison } \\
\text { Fm(?); Holy Ghost Spring } \\
\text { Quadrangle }\end{array}$ & \\
\hline 58 & Salt Spring & $V A-36$ & $3 / 81$ & $\begin{array}{l}\text { Lat } 35^{\circ} 35^{\prime} 52^{\prime \prime} \\
\text { Long } 106^{\circ} 45^{\prime} 34^{\prime \prime}\end{array}$ & $c$ & 15.5 & 7.90 & - & 10500 & $1 / 2$ & $\begin{array}{l}\text { Spring seeps from Chinle Fin, } \\
\text { and Tertiary gravels; San } \\
\text { Ysidro Quadrangle }\end{array}$ & $T 78(A 10)$ \\
\hline 59 & Log Spring & YA-37 & $8 / 79$ & $\begin{array}{l}\text { Lat } 35^{\circ} 38^{\circ} 50^{\prime \prime} \\
\text { Long } 106^{\circ} 50^{\prime} 57^{\prime \prime}\end{array}$ & $m$ & 28.5 & -- & - & -- & $<1$ & $\begin{array}{l}\text { Spring seeps from fault zone } \\
\text { near contact of limestone } \\
\text { and granite; Gilman Quadrangle }\end{array}$ & $T 78(D 4)$ \\
\hline 60 & Owl Spring & $V A-38$ & $3 / 81$ & $\begin{array}{l}\text { Lat } 35^{\circ} 37^{\prime} 44^{\prime \prime} \\
\text { Long } 106^{\circ} 45^{\prime} 48^{\prime \prime}\end{array}$ & $m$ & 16 & 7.22 & - & 620 & 25 & $\begin{array}{l}\text { Spring flows from Madera } \\
\text { Limestone; Gilman Quadrangle }\end{array}$ & $\begin{array}{l}P-H-A ; T 78 \\
(A B)\end{array}$ \\
\hline
\end{tabular}

$a_{m}=$ surface meteoric; $c m=$ carbonated meteoric; $a=$ acid sulphate; $t m=$ thermal meteoric; $d=$ deep geothermal + derivative; $c=$ connate.

${ }^{B} G-G$ is Goff and Grigsby (1982); G et al. is Goff et a1. (1981); G-S is Goff and Sayer (1980); Gr is Griggs (1964); $M$ is Mariner et al. (1977); P77 is Purtymun (1977); P79 is Purtymun (1979); $P-C$ is Purtymun and Cooper (1969); $P-P-0$ is Purtymun et al. (1980); $P-H-A$ is Purtymun et al. (1974); $S$ is Summers (1976); T74 is Trainer (1974); T75 is Trainer (1975); T78 is Trainer (1978); Trainer's sample no. given in parentheses.

CLab values.

$d_{y} s=$ stean fraction, $p^{s}=$ separation pressure. 
TABLE B-II

MAJOR ELEMENT ANALYSES FOR WATERS IN THE JEMEZ MOUNTAINS REGION, NEW MEXICO (VALUES IN mg/l)

\begin{tabular}{|c|c|c|c|c|c|c|c|c|c|c|c|c|c|c|c|c|c|c|c|}
\hline $\begin{array}{l}\text { Map } \\
\text { No. }\end{array}$ & Name & $\begin{array}{l}\text { Field } \\
\text { No. }\end{array}$ & $\begin{array}{l}\text { Temp } \\
(\cdot \mathrm{C})\end{array}$ & $\mathrm{SiO}_{2}$ & $\mathrm{Ca}$ & $\mathrm{Mg}$ & Sr & $\mathrm{Na}$ & $k$ & Li & $\mathrm{HCO}_{3}$ & $\mathrm{SO}_{4}$ & $\mathrm{Cl}$ & $\mathbf{F}$ & $\mathrm{Br}$ & B & TDS & Lca t & $\operatorname{san}$ \\
\hline \multicolumn{20}{|c|}{ Surface and Near-Surface Meteoric Waters } \\
\hline 1 & Gallery Spring & LA-1 & 11 & 43 & 7.0 & 3.3 & 0.05 & 5.8 & 1.4 & 0.02 & 52 & $<5$ & $<1$ & 0.12 & -- & $<0.05$ & 119 & 0.91 & 0.99 \\
\hline 2 & T-3 Well & LA-2 & 13 & 15 & 14.0 & 5.0 & 0.05 & 11.0 & 1.9 & 0.03 & 102 & 5 & 4 & 0.26 & -- & $<0.05$ & 158 & 1.64 & 1.90 \\
\hline 3 & $\mathrm{~T}-2 \mathrm{Hell}$ & $L A-3$ & 11 & 5 & 11.0 & 2.7 & 0.03 & 8.8 & 0.88 & 0.03 & 78 & 5 & 2 & 0.46 & -- & $<0.05$ & 114 & 1.18 & 1.46 \\
\hline 4 & Sacred Spring & LA-4 & 14 & 34 & 22 & 0.45 & 0.42 & 20 & 2.5 & 0.04 & 114 & 7 & 2 & 0.46 & -- & $<0.05$ & 203 & 2.07 & 2.10 \\
\hline 5 & Basalt Spring & LA-5 & 15 & 44 & 26 & 7.6 & 0.12 & 12 & 3.1 & 0.03 & 98 & 18 & 12 & 0.32 & -- & $<0.05$ & 221 & 2.53 & 2.34 \\
\hline 6 & L-6 Well & $L A-6$ & 27 & 33 & 2.8 & 0.15 & 0.05 & 72 & 0.8 & 0.04 & 170 & 6 & 4 & 2.2 & -- & $<0.05$ & 291 & 3.31 & 3.14 \\
\hline 7 & L-1B Well & LA-7 & 30 & 36 & 6.5 & 0.30 & 0.14 & 138 & 2.0 & 0.11 & 326 & 32 & 15 & 2.3 & -- & 0.45 & 559 & 6.42 & 6.55 \\
\hline 8 & L-5 Well & LA-8 & 26.5 & 40 & 7.2 & 0.13 & 0.10 & 52 & 1.3 & 0.04 & 143 & 6 & 3 & 0.98 & -- & $<0.05$ & 254 & 2.67 & 2.61 \\
\hline 9 & L-4 We 11 & LA-9 & 28 & 39 & 10 & 0.22 & 0.07 & 21 & 1.7 & 0.03 & 85 & 5 & 4 & 0.33 & -- & 0.38 & 167 & 1.48 & 1.63 \\
\hline 10 & PM-2 Well & LA-10 & 23.5 & 83 & 8.8 & $3 . \sigma$ & 0.04 & 9.6 & 1.7 & 0.02 & 65 & $<5$ & 3 & 0.19 & -- & 0.25 & 180 & 1.15 & \\
\hline 11 & PM-1 Well & LA-II & 28 & 82 & 26 & 6.8 & 0.14 & 18 & 3.6 & 0.03 & 146 & 6 & 6 & 0.26 & -- & 0.25 & 295 & 2.74 & 2.70 \\
\hline 12 & G-6 Well & LA-12 & 30.5 & 55 & 15 & 2.3 & 0.06 & 15 & 2.0 & $<0.02$ & 94 & 5 & 2 & 0.27 & -- & $<0.05$ & 191 & 1.65 & 1.72 \\
\hline 13 & G-5 Kell & $L A-13$ & 26.5 & 59 & 17 & 3.9 & 0.08 & 11 & 1.8 & $<0.02$ & 93 & 5 & 2 & 0.25 & -- & 0.12 & 193 & 1.70 & 1.70 \\
\hline 14 & G-4 Well & $L A-14$ & 26 & 53 & 16 & 2.5 & 0.07 & 14 & 1.8 & $<0.02$ & 92 & 5 & 2 & 0.27 & -- & 0.12 & 187 & 1.66 & 1.68 \\
\hline 15 & G-3 Well & LA-15 & 29 & 59 & 11 & 1.2 & 0.06 & 22 & 1.6 & $<0.02$ & 93 & 5 & 2 & 0.45 & -- & $<0.05$ & 195 & 1.65 & 1.71 \\
\hline 16 & G-2 Well & LA-16 & 30 & 77 & 11 & 0.61 & 0.08 & 33 & 2.5 & 0.02 & 122 & 5 & 4 & 1.0 & -- & 0.12 & 256 & 2.10 & 2.27 \\
\hline 17 & G-1A Kell & LA-17 & 28 & 78 & 11 & 0.58 & 0.08 & 24 & 2.8 & $<0.02$ & 100 & 5 & 1 & 0.55 & -- & $<0.05$ & 223 & 1.72 & 1.80 \\
\hline 18 & G-1 Well & LA-18 & 26 & 84 & 13 & 0.68 & 0.08 & 22 & 3.1 & $<0.02$ & 97 & 5 & 1 & 0.50 & -- & $<0.05$ & 226 & 1.74 & 1.75 \\
\hline 19 & $\begin{array}{l}\text { Spring, White } \\
\text { Rock Canyon }\end{array}$ & LA-19 & 19 & 71 & 12 & 3.1 & 0.05 & 11 & 1.4 & 0.03 & 74 & $<5$ & $<1$ & 0.45 & - & $<0.05$ & 179 & 1.37 & 1.37 \\
\hline 20 & PM-3 Well & LA-20 & 27.5 & 91 & 26 & 8.7 & 0.12 & 16 & 3.3 & 0.04 & 146 & 6 & 12 & 0.28 & -- & 0.18 & 164 & 2.80 & 2.87 \\
\hline 32 & Pajarito Spring & $V A-29$ & 20 & 67 & 19.6 & $5: 3$ & 0.132 & 11.8 & 2.08 & 0.08 & 100 & 7.5 & 6.4 & 0.46 & -- & 0.05 & 220 & 1.99 & 2.00 \\
\hline
\end{tabular}


TABLE B-II (cont)

\begin{tabular}{|c|c|c|c|c|c|c|c|c|c|c|c|c|c|c|c|c|c|c|c|}
\hline $\begin{array}{l}\text { Map } \\
\text { No. }\end{array}$ & Name & $\begin{array}{l}\text { Field } \\
\text { No. }\end{array}$ & $\begin{array}{l}\text { Temp } \\
\left({ }^{\circ} \mathrm{C}\right)\end{array}$ & $\mathrm{SiO}_{2}$ & $\mathrm{Ca}$ & $\mathrm{Mg}$ & $\mathrm{Sr}$ & $\mathrm{Na}$ & $\mathrm{K}$ & Li & $\mathrm{HCO}_{3}$ & $\mathrm{SO}_{4}$ & $\mathrm{Cl}$ & $F$ & $\mathrm{Br}$ & B & TDS & $\Sigma$ cat & Ean \\
\hline 33 & $\begin{array}{l}\text { Spring, White } \\
\text { Rock Canyon }\end{array}$ & VA-30 & 18 & 63 & 29.3 & 6.9 & 0.225 & 14.4 & 2.4 & 0.07 & -- & 8.8 & 9.8 & 0.48 & -- & 0.07 & 136 & 2.73 & 0.48 \\
\hline 52 & $\begin{array}{l}\text { Unnamed Cold } \\
\text { Spring }\end{array}$ & $V A-58$ & 15 & 65 & 10.6 & 3.4 & 0.049 & 9.4 & 3.1 & 0.024 & 75 & 3.4 & 7.1 & 0.30 & $<0.2$ & $<0.01$ & 178 & 1.30 & 1.52 \\
\hline 53 & $\begin{array}{l}\text { Unnamed cold } \\
\text { Spring }\end{array}$ & $V A-59$ & 17 & 69 & 10.4 & 3.4 & 0.051 & 9.0 & 3.2 & 0.024 & 73 & 2.4 & 7.5 & 0.35 & $<0.2$ & $<0.01$ & 179 & 1.28 & 1.48 \\
\hline 27 & San Antonio Creek & VA-104 & 22.4 & -- & -- & -- & -- & -- & -- & -- & 51 & 8.70 & 1.24 & 1.33 & $<0.10$ & $<0.01$ & -- & -- & -- \\
\hline 28 & San Antonio Creek & VA-24 & 2 & -- & -- & -- & -- & -- & -- & -- & -- & -- & -- & -- & - & -- & -- & -- & -- \\
\hline 28 & San Antonio Creek & $V A-95$ & 3.0 & 56 & 9.8 & 1.56 & 0.06 & 11 & 2.24 & 0.10 & 46.4 & 9.8 & 1.6 & 1.6 & $<0.02$ & $<0.01$ & 140 & 1.17 & 1.09 \\
\hline 28 & San Antonio Creek & YA-127 & 0.5 & 38 & 10 & 2.0 & 0.10 & 10 & 2.4 & 0.06 & 39.0 & 3.5 & 6.1 & 0.97 & -- & -- & 112 & 1.17 & 0.94 \\
\hline 29 & $\begin{array}{l}\text { Jemez River at } \\
\text { Soda Dam }\end{array}$ & VA-52 & 5 & 17 & 11 & 1.4 & 0.038 & 5.6 & 2.0 & 0.040 & 52 & 9.0 & 4.0 & 0.56 & $<0.05$ & 0.09 & 103 & 0.97 & 1.18 \\
\hline 29 & $\begin{array}{l}\text { Jemez River at } \\
\text { Soda Dam }\end{array}$ & $V A-88$ & 1.9 & - & -- & - & -- & -- & - & -- & 107 & 19.8 & 19.5 & 0.96 & $<0.02$ & -- & -- & -- & -- \\
\hline 29 & $\begin{array}{l}\text { Jemez River at } \\
\text { Soda Dam }\end{array}$ & VA-111 & 0.7 & - & $\cdots$ & - & -- & - & -- & -- & 101 & 14.7 & 10.3 & 1.00 & 0.10 & - & -- & -. & -- \\
\hline 29 & $\begin{array}{l}\text { Jemez River at } \\
\text { Soda Dam }\end{array}$ & VA-131 & 9 & 32 & 20 & 2.5 & 0.09 & 17 & 5 & 0.15 & 56.7 & 14.2 & 11.3 & 0.28 & 0.09 & 0.11 & 159 & 2.09 & 1.56 \\
\hline 31 & Panorama Spring & $V A-28$ & 13 & 62 & 101 & 22.9 & 0.720 & 114 & 5.3 & 0.16 & 519 & 56.7 & 21.7 & 0.97 & -- & $<0.1$ & 905 & 12.05 & 10.35 \\
\hline 31 & Panorama Spring & VA-86 & 7.2 & -- & -- & -- & -- & - & -- & -- & 480 & 21.6 & 13.1 & 0.89 & $<0.02$ & -- & -- & -- & -- \\
\hline 31 & Panorama Spring & $V A-136$ & 9 & 51.0 & 64 & 23.9 & 0.47 & 11 & 3 & 0.04 & 477 & 26 & 14.1 & 0.97 & 0.16 & 0.08 & 672 & 5.73 & 8.81 \\
\hline 35 & Sino Spring & $V A-32$ & 21 & -- & -- & -- & -- & - & -- & -- & -- & -- & -- & -- & -- & -- & - & -- & -- \\
\hline 35 & Sino Spring & $V A-63$ & 18 & 78 & 11.2 & 3.6 & 0.06 & 14 & 0.2 & 0.12 & 82 & 5.0 & 6.0 & 0.32 & $<0.4$ & 0.01 & 201 & 1.49 & 1.63 \\
\hline 35 & Sino Spring & $V A-69$ & 22 & 80 & 12.8 & 3.95 & -- & 12 & 0.8 & 0.04 & 77 & 4.3 & 7.7 & 0.46 & $<0.1$ & 0.02 & 199 & 1.51 & 1.59 \\
\hline 35 & Sino Spring & $V A-85$ & 16.9 & -- & -- & -- & - & -- & -- & -- & 75.6 & 4.8 & 3.0 & 0.86 & $<0.02$ & -- & -- & -- & -- \\
\hline 35 & Sino Spring & $V A-102$ & 21.3 & -- & - & -- & -- & -- & -- & -- & 71 & 4.04 & 3.23 & 0.65 & 0.16 & $<0.01$ & - & -- & -- \\
\hline
\end{tabular}




\begin{tabular}{|c|c|c|c|c|c|c|c|c|c|c|c|c|c|c|c|c|c|c|c|}
\hline $\begin{array}{l}\text { Map } \\
\text { No. }\end{array}$ & Name & $\begin{array}{l}\text { Field } \\
\text { No. }\end{array}$ & $\begin{array}{l}\text { Temp } \\
\left({ }^{\circ} \mathrm{C}\right)\end{array}$ & $\mathrm{SiO}_{2}$ & $\mathrm{Ca}$ & Mg & $S r$ & $\mathrm{Na}$ & $k$ & $\mathbf{L i}$ & $\mathrm{HCO}_{3}$ & $\mathrm{SO}_{4}$ & $\mathrm{Cl}$ & $F$ & $B r$ & B & TDS & sca t & $\varepsilon_{\text {an }}$ \\
\hline 38 & $\begin{array}{l}\text { Indian valley } \\
\text { Well }\end{array}$ & $V A-41$ & 17.5 & 77 & 23.5 & 6.2 & 0.160 & 22.0 & 4.78 & 0.044 & 137 & 6.2 & 7.3 & 0.17 & -- & $<0.003$ & 284 & 2.77 & 2.59 \\
\hline 40 & Unnamed Spring & $V A-43$ & 15 & 45 & 5.3 & 1.03 & 0.038 & 3.8 & 3.8 & 0.007 & 11 & 13.1 & 6.9 & 0.33 & $<0.1$ & $<0.01$ & 90.4 & 0.61 & 0.67 \\
\hline 40 & Unnamed Spring & VA-139 & 3 & 34.0 & 8 & 1.6 & 0.09 & 6 & 4 & 0.05 & 28.7 & 11.7 & 1.5 & 0.13 & -- & -- & 95.8 & 0.90 & 0.76 \\
\hline 41 & Unnamed Spring & $V A-44$ & 8 & 48 & 19.0 & 5.0 & 0.120 & 10.6 & 9.08 & 0.002 & 90 & 17.4 & 7.4 & 0.11 & -- & $<0.002$ & 207 & 2.06 & 2.05 \\
\hline 43 & Unnamed Spring & $V A-46$ & 10 & 74 & 10.7 & 1.50 & 0.072 & 11.6 & 2.53 & 0.04 & 57 & 4.4 & 3.5 & 0.44 & -- & $<0.003$ & 166 & 1.23 & 1.15 \\
\hline 44 & Seven Springs & $V A-47$ & 10 & 41 & 12.3 & 1.54 & 0.071 & 7.23 & 2.10 & 0.018 & 49 & 8.7 & 3.6 & 0.21 & -- & $<0.003$ & 126 & 1.11 & 1.10 \\
\hline 44 & Seven Springs & VA-138 & 12 & 30.0 & 11 & 1.4 & 0.05 & 8 & 4 & 0.05 & 53.7 & 5.9 & 1.9 & 0.25 & 0.27 & - & 117 & 1.12 & 1.07 \\
\hline 44 & $\begin{array}{l}\text { Cold Spring west } \\
\text { of Caldera }\end{array}$ & VA-134 & -- & 30 & 12 & 1.5 & 0.06 & 8 & 3 & 0.03 & 53.7 & 5.5 & 1.9 & 0.25 & 0.06 & 0.73 & 117 & 1.15 & 1.06 \\
\hline 45 & $\begin{array}{l}\text { Unnamed Cold } \\
\text { Spring }\end{array}$ & $V A-48$ & 9 & 22 & 33 & 8.7 & 0.150 & 5.9 & 2.1 & 0.003 & 59 & 78 & 6.2 & 0.28 & -- & $<0.01$ & 215 & 2.68 & 2.78 \\
\hline 46 & Eddy's Well & $V A-49$ & 15 & 64 & 56 & 13.8 & 0.288 & 18 & 9.6 & 0.098 & 332 & 28.6 & 8.0 & 0.52 & $<0.1$ & $<0.01$ & 518 & 4.98 & 6.29 \\
\hline 46 & Henson's Well & VA-61 & 19 & 62 & 59 & 8.4 & 0.28 & 36 & 4.6 & 0.136 & 305 & 20.5 & 7.7 & 0.38 & $<0.4$ & 0.01 & 504 & 5.34 & 5.66 \\
\hline 48 & $\begin{array}{l}\text { Unnamed cold } \\
\text { Spring }\end{array}$ & VA-54 & 11 & 30 & 10.0 & 2.0 & 0.064 & 3.5 & 2.9 & 0.002 & 40 & 9.9 & 5.4 & 0.22 & -- & $<0.01$ & 104 & 0.89 & 1.03 \\
\hline 49 & $\begin{array}{l}\text { Unnamed Cold } \\
\text { Spring }\end{array}$ & $V A-55$ & 8.5 & 34 & 5.6 & 1.4 & 0.043 & 2.7 & 1.8 & 0.005 & 24 & 6.0 & 5.5 & 0.21 & -- & $<0.01$ & 80.7 & 0.53 & 0.68 \\
\hline 50 & $\begin{array}{l}\text { Unnamed cold } \\
\text { Spring }\end{array}$ & $V A-56$ & 6.5 & 38 & 8.7 & 3.0 & 0.067 & 5.0 & 2.9 & 0.004 & 39 & 6.7 & 9.8 & 0.20 & $<0.5$ & $<0.01$ & 195 & 0.97 & 1.07 \\
\hline 51 & Apache Spring & VA-57 & 9 & 58 & 10.8 & 4.6 & 0.064 & 6.7 & 3.5 & 0.006 & 57 & 8.3 & 8.0 & 0.27 & $<0.2$ & $<0.01$ & 158 & 1.30 & 1.35 \\
\hline 54 & Turkey Spring & VA -60 & 18 & 61 & 20 & 5.5 & 0.066 & 10.1 & 1.7 & 0.010 & 103 & 4.2 & 8.0 & 0.38 & $<0.2$ & 0.03 & 214 & 1.94 & 2.02 \\
\hline 54 & Turkey Spring & VA-137 & 18 & 57 & 20 & 5.2 & 0.10 & 11 & 3 & 0.04 & 106 & 3.7 & 3.6 & 0.20 & 0.11 & -- & 210 & 1.99 & 1.93 \\
\hline 68 & $\begin{array}{l}\text { Las Conchas } \\
\text { Spring }\end{array}$ & VA-124 & 14 & 53 & 6.2 & 0.92 & 0.04 & 10. & 29 & -- & 40.3 & 2.2 & 1.7 & 1.20 & -- & -- & 118.5 & 0.89 & 0.82 \\
\hline
\end{tabular}


TABLE B-II (cont)

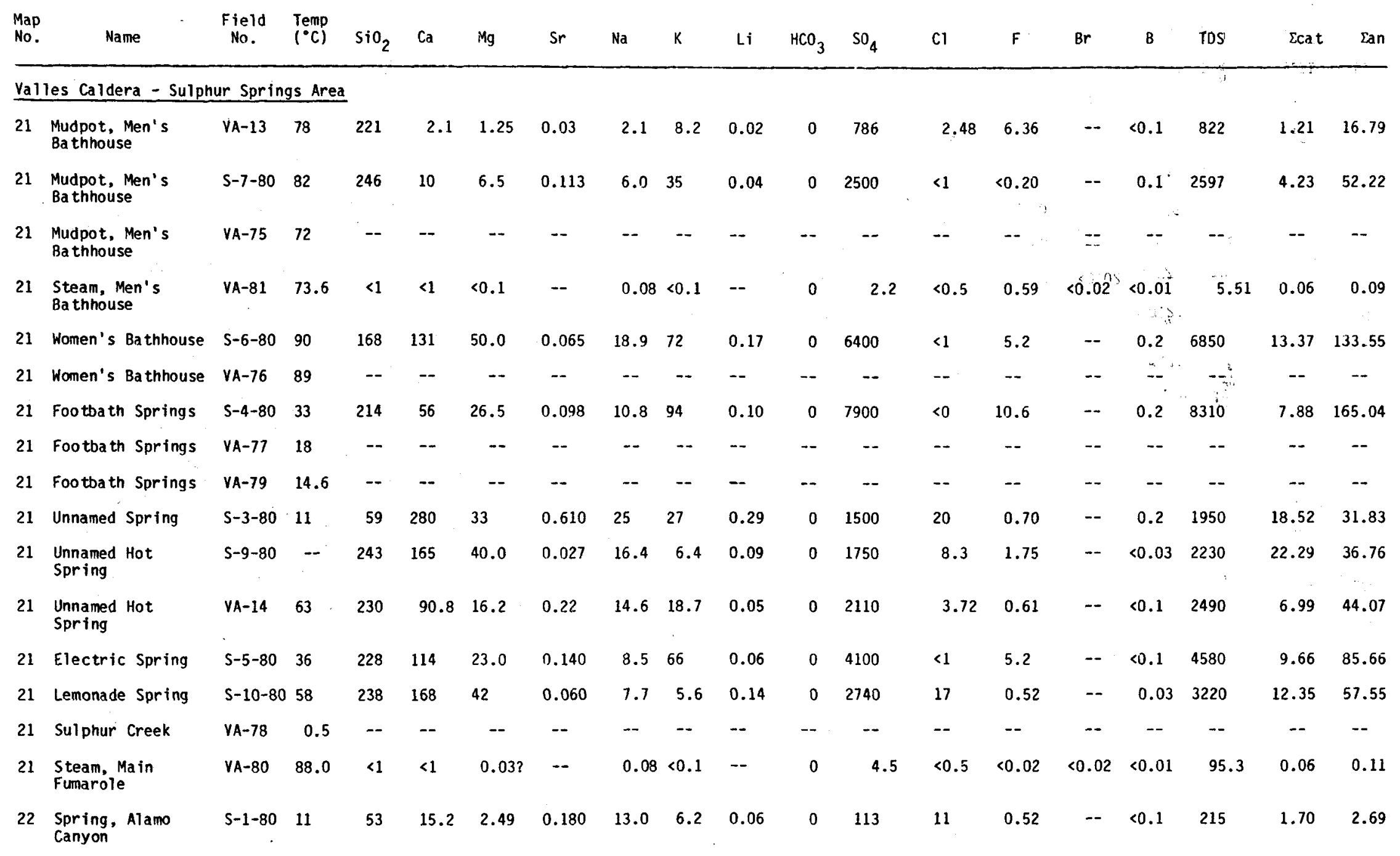




\begin{tabular}{|c|c|c|c|c|c|c|c|c|c|c|c|c|c|c|c|c|c|c|c|}
\hline $\begin{array}{l}\text { Map } \\
\text { No. }\end{array}$ & Name & $\begin{array}{l}\text { Field } \\
\text { No. }\end{array}$ & $\begin{array}{l}\text { Temp } \\
\left({ }^{\circ} \mathrm{C}\right)\end{array}$ & $\mathrm{SiO}_{2}$ & $\mathrm{Ca}$ & $\mathrm{Mg}$ & $\mathrm{Sr}$ & $\mathrm{Na}$ & K & $\mathbf{L i}$ & $\mathrm{HCO}_{3}$ & $\mathrm{SO}_{4}$ & $\mathrm{Cl}$ & $F$ & $\mathrm{Br}$ & 8 & TDS & $\Sigma c a t$ & Ean \\
\hline 22 & $\begin{array}{l}\text { Creek, Alamo } \\
\text { Canyon }\end{array}$ & $s-2-80$ & 11 & 41 & 12.8 & 2.29 & 0.140 & 14.1 & 6.3 & 0.06 & 0 & 109 & 4.9 & 0.23 & - & $<0.1$ & 191 & 1.61 & 2.42 \\
\hline 22 & Bubbling Seep & $V A-23$ & 7 & 51 & 83.5 & 12.0 & 0.68 & 32.8 & 7.9 & 0.08 & 178 & 254 & 7.2 & 0.23 & -- & $<0.1$ & 628 & 6.80 & 8.42 \\
\hline 23 & Bubbling Pool & VA-22 & 0.5 & 44 & 14.1 & 2.75 & 0.40 & 5.8 & 4.5 & 0.4 & 0 & 109 & 7.9 & 0.23 & -- & $<0.1$ & 189 & 1.36 & 2.50 \\
\hline 24 & $\begin{array}{l}\text { Spring, Short } \\
\text { Canyon }\end{array}$ & $S-8-80$ & 8 & 55 & 43 & 5.9 & 0.340 & 7.8 & 7.3 & 0.02 & 0 & 199 & 5.9 & $<0.20$ & -- & $<0.1$ & 325 & 3.16 & 4.32 \\
\hline 63 & GRI Mudpit & $V A-107$ & 1.5 & 19 & 14.6 & 1.11 & 0.14 & 460 & 56 & 5.05 & 133 & 4.6 & 653 & 1.27 & 1.59 & 4.7 & 1360 & 23.00 & 20.97 \\
\hline 63 & $\begin{array}{l}\text { GRI Well at } \\
4800 \mathrm{ft}\end{array}$ & $V A-113$ & 214 & 395 & 80.5 & 2.89 & 2.29 & 2800 & 470 & 28.2 & 360 & 33 & 4350 & 6.9 & 14 & 39.3 & 8580 & 142.20 & 129.65 \\
\hline 63 & $\begin{array}{l}\text { GRI Well at } \\
4800 \mathrm{ft}\end{array}$ & VA-114 & 214 & -- & 64.3 & 3.40 & 2.54 & 2880 & 499 & 37.4 & - & -- & -- & -- & -- & 33.2 & -- & -- & -- \\
\hline 63 & $\begin{array}{l}\text { GRI Well at } \\
6300 \mathrm{ft}\end{array}$ & $V A-116$ & 232.6 & 450 & 46.0 & 0.45 & 1.98 & 5890 & 1020 & 68 & 382 & 95 & 9960 & 13.8 & 27 & 96.2 & 18100 & 294.55 & 289.90 \\
\hline Val & les Caldera - Ring & Fracture & Zone & & & & & & & & & & & & & & & & \\
\hline 25 & Spence Hot Spring & $V A-1$ & 45 & 66 & 5.5 & 1.9 & 0.03 & 50 & 1.3 & 0.66 & 144 & 16 & 8 & 0.55 & -- & 0.15 & 294 & 2.73 & 2.95 \\
\hline 25 & Spence Hot Spring & $V A-68$ & 42 & 69 & 7.2 & 1.87 & 0.02 & 52 & 1.6 & 0.71 & 135 & 18 & 11 & 0.59 & $<0.1$ & 0.15 & 297 & 2.92 & 2.93 \\
\hline 25 & Spence Hot Spring & VA-72 & 42 & 69 & 6.4 & 1.76 & 0.042 & 44 & 1.4 & 0.74 & 138 & 2.8 & 11.2 & 0.69 & $<0.02$ & 0.17 & 276 & 2.52 & 2.67 \\
\hline 25 & Spence Hot Spring & $V A-83$ & 41.6 & -- & -- & -- & -- & -- & -- & -- & 135 & 18.0 & 7.2 & 0.90 & $0.11 ?$ & -- & 161 & -- & -- \\
\hline 25 & Spence Hot Spring & VA-105 & 42.5 & -- & -- & -- & -- & -- & -- & -- & 134 & 18.4 & 7.91 & 0.80 & 0.15 & 0.02 & 161 & -- & -- \\
\hline 25 & Spence Hot Spring & VA-120 & 42.3 & 66 & 5.9 & 1.57 & 0.08 & 50 & 1.4 & 0.53 & 140 & 17.1 & 8.2 & 0.76 & 0.10 & 0.12 & 292 & 2.72 & 2.92 \\
\hline 25 & $\begin{array}{l}\text { Little Spence Hot } \\
\text { Spring }\end{array}$ & $V A-2$ & 34 & 67 & 8.8 & 1.9 & 0.04 & 56 & 1.5 & 0.66 & 152 & 25 & 7 & 0.70 & -- & 0.13 & 321 & 3.17 & 3.25 \\
\hline 26 & McCauley Spring & $V A-3$ & 31 & 56 & 8.5 & 4.9 & 0.02 & 18 & 0.8 & 0.24 & 86 & 7 & 6 & 0.85 & -- & 0.24 & 189 & 1.67 & 1.77 \\
\hline 26 & McCauley Spring & $V A-87$ & 31.5 & -- & $\cdots$ & $\because$ & -- & -- & -- & -- & 80.5 & 6.6 & 3.2 & 1.31 & $<0.02$ & -- & -- & -- & -- \\
\hline 26 & McCauley Spring & $V A-119$ & 31.9 & 54 & 8.5 & 4.32 & 0.04 & 20 & 1.0 & 0.22 & 86.6 & 5.8 & 4.4 & 0.95 & -- & -- & 186 & 1.71 & 1.71 \\
\hline 27 & $\begin{array}{l}\text { San Antonio Hot } \\
\text { Spring }\end{array}$ & $V A-4$ & 42 & 79 & 2.3 & 0.30 & 0.02 & 21 & 1.7 & $<0.02$ & 56 & 7 & 2 & 0.80 & $\therefore$ & $<0.05$ & 170 & 1.10 & 1.16 \\
\hline
\end{tabular}


TABLE B-II (cont)

\begin{tabular}{|c|c|c|c|c|c|c|c|c|c|c|c|c|c|c|c|c|c|c|c|}
\hline $\begin{array}{l}\text { Map } \\
\text { No. }\end{array}$ & Name & $\begin{array}{l}\text { Field } \\
\text { No. }\end{array}$ & $\begin{array}{l}\text { Temp } \\
\left({ }^{\circ} \mathrm{C}\right)\end{array}$ & $\mathrm{SiO}_{2}$ & $\mathrm{Ca}$ & $\mathrm{Mg}$ & $s r$ & $\mathrm{Na}$ & $k$ & Li & $\mathrm{HCO}_{3}$ & $\mathrm{SO}_{4}$ & $\mathrm{Cl}$ & $F$ & $\mathrm{Br}$ & B & TDS & Ecat & Ean \\
\hline 27 & $\begin{array}{l}\text { San Antonio Hot } \\
\text { Spring }\end{array}$ & VA-96 & 40.8 & 80 & 2.7 & 0.28 & 0.02 & 21 & 1.80 & 0.12 & 50.0 & 7.6 & 2.2 & 2.2 & $<0.02$ & $<0.01$ & 168 & 1.13 & 1.16 \\
\hline 27 & $\begin{array}{l}\text { San Antonio Hot } \\
\text { Spring }\end{array}$ & VA-128 & 41.3 & 74 & 3 & 0.5 & 0.06 & 23 & 1.8 & 0.06 & 57.3 & 9.5 & 7.0 & 0.79 & -- & -- & 167 & 1.25 & 1.38 \\
\hline 28 & Bathhouse Spring & $V A-20$ & 38 & 96 & 4.98 & 0.40 & 0.02 & 24.5 & 3.7 & 0.06 & 71 & 15 & 2.4 & 1.6 & -- & $<0.1$ & 220 & 1.45 & 1.63 \\
\hline 28 & Bathhouse Spring & VA-94 & 37.4 & 103 & 5.3 & 0.40 & 0.04 & 27 & 4.04 & 0.09 & 61.0 & 12.7 & 8.6 & 1.84 & $<0.02$ & $<0.01$ & 261 & 1.59 & 1.60 \\
\hline 28 & Bathhouse Spring & VA-126 & 38.1 & 105 & 6 & 1.0 & 0.09 & 27 & 4.2 & 0.08 & 75.6 & 15.2 & 6.9 & 1.46 & 0.2 & - & 498 & 1.68 & 1.83 \\
\hline 34 & Battleship Seep & $V A-31$ & 19 & 18 & 10.2 & 71.0 & 0.146 & 613 & 42.0 & 3.22 & 1745 & 373 & 284 & 4.95 & -- & 4.15 & 3170 & 34.59 & 44.64 \\
\hline 34 & Battleship Seep & VA-50 & 11 & 17 & 35 & 46 & 0.810 & 940 & 47 & 4.0 & 1980 & 290 & 323 & 5.75 & 0.78 & 4.47 & 3690 & 46.14 & 47.11 \\
\hline 34 & Battleship Seep & $V A-133$ & 12 & 16.5 & 31 & 42.8 & 1.78 & 900 & 50 & 4.3 & 1966 & 295 & 299 & 5.73 & 0.89 & 4.45 & 3620 & 46.14 & 47.11 \\
\hline 34 & $\begin{array}{l}\text { East Fork, Jemez } \\
\text { River }\end{array}$ & VA-84 & 4.4 & - & - & -- & -- & -- & -- & -- & 50.0 & 4.6 & 1.9 & 1.07 & $<0.02$ & -- & -- & - & -- \\
\hline 34 & $\begin{array}{l}\text { East Fork, Jemez } \\
\text { River }\end{array}$ & $V A-106$ & -- & $\cdots$ & -- & -- & -- & -- & -- & -- & 43 & 3.80 & 2.36 & 0.55 & $<0.10$ & $<0.10$ & -- & -- & -- \\
\hline 39 & $\begin{array}{l}\text { Valle Grande } \\
\text { Spring }\end{array}$ & $V A-42$ & 15 & 52 & 6.4 & 1.66 & 0.030 & 1.7 & 8.0 & 0.030 & 34 & 2.6 & 9.1 & 0.39 & -- & $<0.01$ & 116 & 0.74 & 0.89 \\
\hline 39 & $\begin{array}{l}\text { Valle Grande } \\
\text { Spring }\end{array}$ & $V A-82$ & 13.6 & -- & -- & -- & -- & -- & -- & -- & 34.2 & 2.3 & 0.8 & 0.26 & $<0.02$ & -- & -- & -- & -- \\
\hline 39 & $\begin{array}{l}\text { Valle Grande } \\
\text { Spring }\end{array}$ & VA-117 & 13.8 & -- & -- & -- & -- & -- & -- & -- & 46.4 & 1.50 & 1.6 & 0.26 & $<0.1$ & -- & -- & - & -- \\
\hline 42 & Horseshoe Spring & $V A-45$ & 12 & 46 & 19.0 & 3.4 & 0.083 & 30.7 & 3.00 & 0.088 & 145 & $6: 2$ & 6.1 & 0.21 & $\cdots$ & 0.003 & 260 & 2.65 & 2.69 \\
\hline 42 & Horseshoe Spring & $V A-118$ & 11.3 & 56 & 28.8 & 4.48 & 0.13 & 35 & 3.1 & -- & 151 & 5.14 & 4.0 & 0.38 & 0.1 & -- & 288 & 3.41 & 2.72 \\
\hline 61 & $\mathrm{PCl}$ at $225 \mathrm{ft}$ & PC1-7 & - & 52 & 13.8 & 4.4 & 0.24 & 29 & 4.6 & -- & 118 & 6.7 & 5.2 & 0.43 & -- & 0.15 & 235 & 2.43 & 2.24 \\
\hline 61 & PC1 at $365-391 \mathrm{ft}$ & PCl-8 & -- & 53 & 8.9 & 2.4 & 0.15 & 30 & 3.8 & $\cdots$ & 110 & 6.2 & 5.3 & 0.27 & -- & 0.21 & 220 & 2.05 & 2.10 \\
\hline 61 & $\mathrm{PC1}$ at $685-691 \mathrm{ft}$ & PCl-9 & - & 16 & 6.0 & 3.8 & 0.09 & 366 & 6.0 & -- & 852 & 24.5 & 5.0 & 2.15 & - & 0.74 & 1280 & 16.69 & 14.73 \\
\hline 61 & PC1 at $943 \mathrm{ft}$ & $P C 1-10$ & -- & 72 & 10.3 & 4.2 & 0.17 & 433 & 6.6 & 0.20 & 686 & 151 & 108 & 2.43 & 0.18 & 0.74 & 1480 & 19.89 & 17.56 \\
\hline 61 & $\mathrm{PCl}$ at $1100 \mathrm{ft}$ & $\mathrm{PC} 1-11$ & -- & 30 & 78 & 73 & 1.24 & 740 & 55 & 1.64 & 2636 & 24.5 & 50.0 & 6.78 & 0.29 & 4.24 & 3700 & 43.76 & 45.49 \\
\hline
\end{tabular}




\begin{tabular}{|c|c|c|c|c|c|c|c|c|c|c|c|c|c|c|c|c|c|c|c|}
\hline $\begin{array}{l}\text { Map } \\
\text { No. }\end{array}$ & Name & $\begin{array}{l}\text { Field } \\
\text { No. }\end{array}$ & $\begin{array}{l}\text { Temp } \\
(\cdot \mathrm{C})\end{array}$ & $\mathrm{SiO}_{2}$ & $\mathrm{Ca}$ & $\mathrm{Mg}$ & $\mathrm{Sr}$ & $\mathrm{Na}$ & K & Li & $\mathrm{HCO}_{3}$ & $\mathrm{SO}_{4}$ & $\mathrm{Cl}$ & $F$ & $\mathrm{Br}$ & B & TDS & $\Sigma_{\text {cat }}$ & $\Sigma_{\text {an }}$ \\
\hline 61 & PCI at $1712 \mathrm{ft}$ & $P C 1-1$ & -- & 225 & 762 & 52.8 & 3.52 & 1390 & 153 & 10.8 & 1133 & 2157 & 1602 & 1.06 & 4.3 & 13.4 & 7510 & 108.34 & 08.72 \\
\hline 61 & PCl at $1937 \mathrm{ft}$ & PC1-2 & -- & 165 & 2.6 & 0.12 & 0.12 & 2480 & 127 & 2.7 & $504^{a}$ & 1614 & 1700 & 3.29 & 5.0 & 6.47 & 4510 & 111.67 & 81.73 \\
\hline 61 & PC1 at $1987 \mathrm{ft}$ & PC1-3 & -- & 7 & 507 & 0.08 & 6.50 & 3030 & 189 & 1.8 & $24^{a}$ & 4210 & 2051 & 0.79 & 4.8 & 1.91 & 10000 & 162.23 & 145.54 \\
\hline 61 & $\mathrm{PC1}$ at $1953 \mathrm{ft}$ & $P C 1-4$ & - & 101 & 545 & 24.6 & 2.32 & 2480 & 120 & 2.9 & 468 & 4105 & 1819 & 0.37 & 5.0 & 7.98 & 9680 & 140.61 & 144.46 \\
\hline 61 & $\mathrm{PC1}$ at $2036 \mathrm{ft}$ & PC1-5 & -- & 108 & 30.0 & 3.6 & 0.30 & 2060 & 78 & 0.64 & 642 & 584 & 2648 & 2.30 & 3.3 & 10.40 & 6170 & 93.50 & 97.49 \\
\hline 71 & PC2 at $587-600 \mathrm{ft}$ & PC2-1 & 30 & 16 & 5.1 & 3.1 & 0.08 & 257 & 7.0 & 0.06 & 715 & 44.7 & 3.3 & 1.79 & $<0.01$ & 0.99 & 1058 & 11.91 & 13.51 \\
\hline 71 & $P C 2$ at $630-635 \mathrm{ft}$ & $P C 2-2$ & 30 & 14 & 4.4 & 2.5 & 0.07 & 303 & 6.2 & 0.05 & 749 & 45.8 & 3.9 & 2.17 & $<0.01$ & 1.00 & 1152 & 14.08 & 14.41 \\
\hline 71 & PC2 at $1086 \mathrm{ft}$ & PC2-3 & -- & 10 & 5.6 & 2.8 & 0.06 & 290 & $<2$ & $<0.01$ & 537 & 80.5 & 64.5 & 3.08 & $<0.05$ & 0.63 & 1024 & 14.12 & 13.29 \\
\hline 71 & PC2 at $1350 \mathrm{ft}$ & PC2-4 & 35 & 32 & 80 & 80.8 & 0.49 & 403 & 20 & 0.56 & 1698 & 78.5 & 36.2 & 2.07 & 0.22 & 1.28 & 2403 & 28.90 & 30.71 \\
\hline 71 & $\mathrm{PC2}$ at $1360 \mathrm{ft}$ & PC2-5 & 38 & 43 & 96 & 96.2 & 0.67 & 410 & 30 & 1.16 & 1886 & 26.3 & 37.8 & 2.28 & 0.20 & 1.76 & 2590 & 31.63 & 32.78 \\
\hline 71 & $\mathrm{PC2}$ at $1335 \mathrm{ft}$ & PC2-6 & 40 & 66 & 646 & 215 & 3.46 & 670 & 69 & 1.86 & 1102 & 2807 & 57 & 1.34 & $<0.1$ & 2.30 & 5577 & 81.72 & 78.70 \\
\hline $\begin{array}{l}71 \\
\text { vall } \\
\end{array}$ & $\begin{array}{l}\text { PC2 at } 1490 \mathrm{ft} \\
\text { les Caldera - Baca }\end{array}$ & $\begin{array}{l}\text { PC2-7 } \\
\text { Geother }\end{array}$ & $\begin{array}{r}41 \\
\operatorname{mal} F \\
\end{array}$ & 30 & 96 & 61.7 & 1.23 & 562 & 50 & 2.96 & 1729 & 178 & 44.6 & 6.50 & 0.34 & 2.97 & 2737 & 36.20 & 33.80 \\
\hline 62 & $\begin{array}{l}\text { Redondo Creek } \\
\text { at Union Gate }\end{array}$ & VA-129 & 4 & 25 & 10 & 1.4 & 0.06 & 9 & 4 & 0.06 & 26.8 & 12.5 & 10.7 & 0.13 & 0.09 & 0.06 & 100 & 1.12 & 1.01 \\
\hline 64 & Baca Well $\# 4$ & $B A-2$ & 294 & 720 & 3.6 & $<0.01$ & 0.10 & 1570 & 285 & 20.6 & 215 & 49 & 2640 & 0.0 & 7.80 & - & 4769 & 76.99 & 80.16 \\
\hline 64 & Baca Well :4 & $B A-5$ & 297 & 760 & 3.7 & $<0.01$ & 0.10 & 1560 & 280 & 20.0 & 190 & 49 & 2670 & 0.0 & 7.83 & 17.9 & 4719 & 74.58 & 80.61 \\
\hline 65 & Baca Hell 13 & $B A-1$ & 278 & 640 & 3.5 & - & 0.21 & 1550 & 255 & 24.7 & 221 & 49 & 2501 & 9.4 & 7.00 & 18.6 & 4529 & 73.00 & 76.41 \\
\hline 65 & Baca Well 13 & $B A-4$ & 279 & 680 & 4.3 & 0.19 & 0.22 & 1540 & 255 & 20.5 & 236 & 47 & 2594 & 9.6 & 7.01 & 18.0 & 4644 & 73.44 & 79.20 \\
\hline 66 & Baca Well 15 & $B A-7$ & 267 & 680 & 13.6 & $<0.01$ & 0.25 & 1950 & 330 & 23.1 & 89 & 45 & 3257 & 6.9 & 9.59 & 25.4 & 5735 & 97.07 & 95.79 \\
\hline 66 & Baca Well \#15 & $B A-8$ & 326 & 689 & 14.2 & $<0.01$ & 0.23 & 1910 & 350 & 24.3 & 75 & 37 & 3302 & 6.8 & 9.20 & 25.0 & 5783 & 98.15 & 96.52 \\
\hline 66 & Baca Well $\# 19$ & $B A-9$ & 223 & $565 ?$ & 13.1 & 0.02 & 0.30 & 1920 & 310 & 25.6 & 139 & 48 & 3340 & 6.9 & 10.85 & 26.8 & 6147 & 96.44 & 107.70 \\
\hline 67 & Baca Well $\# 24$ & $B A-3$ & 260 & 620 & 17.2 & $<0.01$ & 0.111 & 1870 & 230 & 23.2 & 89 & 48 & 3151 & 6.4 & 9.36 & 26.3 & 5382 & 88.06 & 92.68 \\
\hline 67 & Baca Well 24 & BA-6 & 261 & 600 & 17.1 & $<0.01$ & 0.12 & 1850 & 235 & 23.2 & 90 & 48 & 3128 & 6.6 & 9.42 & 26.5 & 5339 & 86.92 & 92.14 \\
\hline
\end{tabular}


TABLE B-II (cont)

\begin{tabular}{|c|c|c|c|c|c|c|c|c|c|c|c|c|c|c|c|c|c|c|c|}
\hline $\begin{array}{l}\text { Map } \\
\text { No. }\end{array}$ & Name & $\begin{array}{c}\text { Field } \\
\text { No. }\end{array}$ & $\begin{array}{l}\text { Temp } \\
\left({ }^{\circ} \mathrm{C}\right)\end{array}$ & $\mathrm{SiO}_{2}$ & $\mathrm{Ca}$ & $\mathrm{Mg}$ & $\mathrm{Sr}$ & $\mathrm{Na}$ & $k$ & $L \mathbf{i}$ & $\mathrm{HCO}_{3}$ & $\mathrm{SO}_{4}$ & $\mathrm{Cl}$ & $\mathbf{F}$ & $\mathrm{Br}$ & $B$ & TOS & $\Sigma$ cat & Ean \\
\hline
\end{tabular}

\section{Valles Caldera - Soda Dam and Jemez Springs Area}

29 Soda Dam Spring.

29 Soda Dam Spring

29 Soda Dam Spring

29 Soda Dam Spring

VA-6 47

$\begin{array}{lll}43 & 328 & 26\end{array}$

$\begin{array}{lll}1.38 & 1010 \quad 174\end{array}$

$13.2 \quad 886$

$37 \quad 1480$

$4.1 \quad--11.5 \quad 4014$

$68.82 \quad 57.26$

$\begin{array}{llllllllll}V A-9 & 48 & 50 & 340 & 24.4 & 1.50 & 938 & 183 & 13.2 & 1510\end{array}$

38.41500

$3.67 \quad--\quad 13.8 \quad 4620$

66.3968 .06

$\begin{array}{llllllllll}V A-26 & 47 & 46 & 429 & 21.4 & 2.02 & 920 & 177 & 13.6 & 1490\end{array}$

49.41460

$3.57 \quad--\quad 12.8 \quad 4630$

$69.70 \quad 66.82$

29 Soda Dam Spring

$\begin{array}{llllllll}46 & 314 & 24 & 0.890 & 990 & 183 & 13.5 & 1000\end{array}$

39.11520

$\begin{array}{llll}3.55 & 3.84 & 15.0 & 4150\end{array}$

$67.36 \quad 60.27$

29 Soda Dam Spring

$\begin{array}{llllllll}44 & 300 & 25 & 1.48 & 825 & 120 & 13.7 & 1250\end{array}$

36.11560

$\begin{array}{llll}2.8 & 6.7 & 13.9 & 4200\end{array}$

$57.98 \quad 65.39$

VA-64 47

$\begin{array}{llllllll}47 & 331 & 23.8 & 0.56 & 860 & 170 & 13.5 & 1390\end{array}$

29 Soda Dam Spring

$\begin{array}{llllllll}48 & 346 & 24.6 & 1.20 & 840 & 186 & 13.7 & 1500\end{array}$

$41 \quad 1480$

$\begin{array}{llll}5.0 & 5.6 & 13.4 & 4380\end{array}$

$62.21 \quad 65.65$

29 Soda Dam Spring

VA-73 47

$36.7 \quad 1570$

$\begin{array}{llll}3.6 & 5.6 & 8.5 ? \quad 4539\end{array}$

$62.95 \quad 70.40$

29 Soda Dam Spring

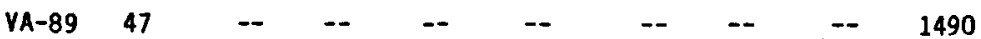

35.51480

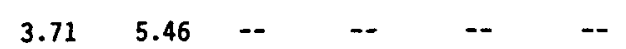

29 Soda Dam Spring

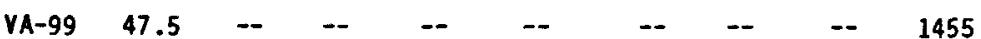

$37.5 \quad 1614$

$\begin{array}{llll}3.68 & 6.0 & 14.1 & --\end{array}$

$--$

29 Soda Dam Spring

$\begin{array}{llllllll}\text { VA-109 } & 46.8 & 47 & 245 & 17.4 & 1.39 & 1030 & 160\end{array}$

$12.7 \quad 1458$

$34 \quad 1536$

$\begin{array}{llll}3.28 & 4.07 & 15.7 & 4570\end{array}$

64.4168 .11

29 Soda Dam Spring

$35 \quad 1477$

$\begin{array}{llll}3.51 & 4.6 & 13.9 \quad 4590\end{array}$

$67.28 \quad 66.97$

29 Soda Dam Spring

$13.8 \quad 1488$

$34 \quad 1480$

$\begin{array}{llll}3.33 & 4.6 & 12.1 & 4570\end{array}$

$66.73 \quad 67.02$

29 Grotto Spring

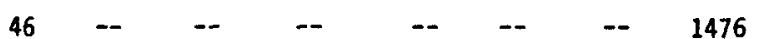

$35 \quad 1567$

$\begin{array}{llll}3.85 & 4.6 & -- & --\end{array}$

$--$

29 Outfall of Soda

$\begin{array}{llllllllll}V A-5 & 38 & 38 & 324 & 27 & 1.40 & 1000 & 174 & 13.2 & 834\end{array}$

$41 \quad 1480$

$4.0 \quad--\quad 11.6 \quad 3950$

$68.27 \quad 56.48$ Dan Spring

29 Hidden Warm

VA-65

$36 \quad 138 \quad 26$

$\begin{array}{lll}1.27 & 943 \quad 160\end{array}$

$14.3 \quad 610$

40.51590

$\begin{array}{llll}2.5 & 6.3 & 14.6 & 3580\end{array}$

$56.23 \quad 55.82$

Spring

VA-27 29

44376

$\begin{array}{llllll}18.8 & 1.90 & 720 & 141 & 10.8 & 1400\end{array}$

69.11195

$2.71 \quad-\quad 10.6 \quad 3990$

$56.81 \quad 58.24$

29 Hidden Warm

VA-90 32

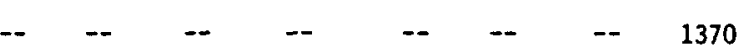

48.31240

3.31

9. Hidden Warm

VA-110 32.3

$\begin{array}{llllllll}43 & 226 & 15.3 & 1.34 & 817 & 130 & 10.5 & 1324\end{array}$

$53 \quad 1294$

$\begin{array}{llll}3.21 & 3.27 & 13.4 & 3930\end{array}$

$52.93 \quad 59.48$

29 Hidden Warm

VA-141 32.2

$\begin{array}{llllllll}43 & 305 & 20.0 & 2.55 & 780 & 125 & 11.1 & 1425\end{array}$

$49 \quad 1240$

$\begin{array}{llll}3.04 & 3.8 & 10.6 & 4020\end{array}$

$55.61 \quad 59.52$

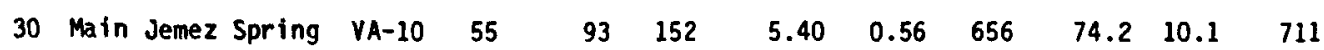

40.9904

$5.19 \quad--\quad 7.9 \quad 2660$

$39.93 \quad 38.28$

$\begin{array}{lllllllllll}30 & \text { Main Jemez Spring VA-18 } & 35.5 & 85 & 115 & 4.52 & 0.60 & 690 & 74.0 & 9.00 & 699\end{array}$

45.4968

$5.19 \quad--\quad 8.0 \quad 2700$

$39.32 \quad 39.98$ 


\begin{tabular}{|c|c|c|c|c|c|c|c|c|c|c|c|c|c|c|c|c|c|c|c|}
\hline $\begin{array}{l}\text { Map } \\
\text { No. }\end{array}$ & Name & $\begin{array}{l}\text { Field } \\
\text { No. }\end{array}$ & $\begin{array}{l}\text { Temp } \\
\left({ }^{\circ} \mathrm{C}\right)\end{array}$ & $\mathrm{SiO}_{2}$ & $\mathrm{Ca}$ & $\mathrm{Mg}$ & $\mathrm{Sr}$ & $\mathrm{Na}$ & $x$ & Li & $\mathrm{HCO}_{3}$ & $\mathrm{SO}_{4}$ & $\mathrm{Cl}$ & $F$ & $\mathrm{Br}$ & B & TOS & Icat & Lan \\
\hline 30 & Main Jemez Spring & VA-93 & 46.3 & -- & -- & -- & -- & -- & -- & -- & 720 & 45.5 & 926 & 5.18 & 2.86 & -- & -- & -- & - \\
\hline 30 & Main Jemez Spring & $V A-122$ & 74.9 & 91 & 126 & 4.57 & 0.57 & 583 & 62 & 6.8 & 698 & 39 & 909 & 6.4 & 2.2 & 7.11 & 2540 & 34.60 & 38.23 \\
\hline 30 & Main Jemez Spring & VA-143 & 74.7 & 91 & 130 & 4.55 & 1.18 & 610 & 58 & 8.4 & 746 & 42 & 932 & 4.64 & 2.7 & 6.4 & 2640 & 36.10 & 39.64 \\
\hline 30 & Main Jemez Spring & $V A-147$ & -- & 90 & -- & -- & -- & -- & -- & -- & -- & 706 & 922 & 2.4 & 3.9 & 5.18 & -- & -- & -- \\
\hline 30 & $\begin{array}{l}\text { Jemez Springs } \\
\text { Geothermal We } 11\end{array}$ & $V A-121$ & 73.3 & 89 & 121 & 4.55 & 0.67 & 583 & 62 & 6.8 & 698 & 39 & 909 & 6.4 & 2.2 & 7.11 & 2530 & 34.35 & 38.23 \\
\hline 30 & $\begin{array}{l}\text { Jemez Springs } \\
\text { Geothermal Well }\end{array}$ & VA-144 & 72.2 & 94 & 126 & 4.23 & 1.22 & 630 & 61 & 8.5 & 714 & 41 & 941 & 5.38 & 2.6 & 6.5 & 3005 & 36.83 & 39.38 \\
\hline 30 & Jemez We $11 / 24$ m & VA-19 & 68 & 70 & 122 & 5.76 & 0.54 & 546 & 61.6 & 6.96 & 642 & 45.0 & 705 & 4.42 & -- & 6.1 & 2220 & 32.90 & 31.58 \\
\hline 30 & Jemez Hel1/24 m & VA-25 & 73.3 & 79 & 180 & 4.60 & 0.86 & 610 & 68.0 & 8.4 & 705 & 53.0 & 836 & 3.52 & -- & 6.8 & 2560 & 38.85 & 36.43 \\
\hline 30 & Jemez We11/152 m & $V A-15$ & 60.5 & 24 & 120 & 9.31 & 0.40 & 185 & 29.9 & 2.27 & 479 & 38.0 & 243 & 3.30 & -- & 2.2 & 1140 & 15.90 & 15.67 \\
\hline 30 & Jemez We11/152 m & VA-21 & 61 & 36 & 122 & 9.25 & 0.40 & 193 & 35.4 & 3.60 & 492 & 49.9 & 281 & 3.50 & -- & 2.5 & 1200 & 16.68 & 17.21 \\
\hline 30 & $\begin{array}{l}\text { Travertine Mound } \\
\text { Spring }\end{array}$ & VA-7 & 70 & 93 & 182 & 4.56 & 0.60 & 614 & 75.2 & 8.20 & 723 & 36.1 & 829 & 5.21 & -- & 7.8 & 7580 & 39.28 & 36.26 \\
\hline 30 & $\begin{array}{l}\text { Travertine Mound } \\
\text { Spring }\end{array}$ & VA-17 & 72 & 83 & 114 & 4.48 & 0.54 & 612 & 70.3 & 8.46 & 714 & 43.2 & 936 & 5.05 & -- & 7.9 & 2600 & 35.71 & 39.27 \\
\hline 30 & $\begin{array}{l}\text { Travertine Mound } \\
\text { Spring }\end{array}$ & VA-66 & 72 & 92 & 122 & 5.4 & 0.59 & 558 & 62 & 9.0 & 436 & 42.4 & 910 & 4.0 & 2.6 & 7.0 & 1700 & 33.70 & 33.91 \\
\hline 30 & $\begin{array}{l}\text { Travertine Mound } \\
\text { Spring }\end{array}$ & VA-71 & 72 & 92 & 135 & 4.75 & 0.337 & 540 & 73 & 8.7 & 712 & 41 & 894 & 4.4 & 2.8 & 7.19 & 2520 & 33.75 & 37.97 \\
\hline 30 & $\begin{array}{l}\text { Travertine Mound } \\
\text { Spring }\end{array}$ & $V A-91$ & 72.3 & -- & -- & -- & -- & -- & -- & - & 715 & 39.8 & 869 & 5.13 & 3.01 & -- & -- & - & -- \\
\hline 30 & $\begin{array}{l}\text { Travertine Mound } \\
\text { Spring }\end{array}$ & VA-123 & 72.6 & 90 & 129 & 4.49 & 0.57 & 596 & 62 & 6.1 & 697 & 38 & 906 & 6.3 & 2.3 & 7.02 & 2550 & 35.21 & 38.10 \\
\hline 30 & $\begin{array}{l}\text { Travertine Mound } \\
\text { Spring }\end{array}$ & VA-142 & 72.9 & 92 & 132 & 4.3 & 1.22 & 610 & 58 & 8.6 & 757 & 40 & 917 & 4.31 & 2.7 & 6.7 & 2630 & 36.21 & 39.33 \\
\hline 30 & Buddhist Spring & $V A-8$ & 49 & 81 & 154 & 9.57 & 0.56 & 458 & 53.0 & 7.56 & 697 & 37.6 & 653 & 3.86 & -- & 5.7 & 2160 & 30.85 & 30.83 \\
\hline 30 & Buddhist Spring & $V A-16$ & 50 & 72 & 128 & 7.50 & 0.52 & 494 & 57.8 & 6.06 & 708 & 40.6 & 653 & 3.76 & -- & 5.7 & 2550 & 30.85 & 31.07 \\
\hline 30 & Buddhist Spring & VA-92 & 43.2 & -- & -- & -- & -- & -- & - & -- & 660 & 35.1 & 617 & 3.66 & 1.85 & -- & -- & - & -- \\
\hline
\end{tabular}




\section{TABLE B-II (cont)}

\begin{tabular}{|c|c|c|c|c|c|c|c|c|c|c|c|c|c|c|c|c|c|c|c|}
\hline $\begin{array}{l}\text { Map } \\
\text { No. }\end{array}$ & Name & $\begin{array}{l}\text { Field } \\
\text { No. }\end{array}$ & $\begin{array}{l}\text { Temp } \\
\left({ }^{\circ} \mathrm{C}\right)\end{array}$ & $\mathrm{SiO}_{2}$ & Ca & $\mathrm{Mg}$ & $\mathrm{Sr}$ & $\mathrm{Na}$ & K & Li & $\mathrm{HCO}_{3}$ & $\mathrm{SO}_{4}$ & Cl & $\mathbf{F}$ & $\mathrm{Br}$ & B & TDS & $\Sigma_{\text {cat }}$ & $\Sigma_{\text {an }}$ \\
\hline 30 & Unnamed Spring & $Y A-12$ & 49 & 100 & 129 & 7.82 & 0.64 & 609 & 70.0 & 8.18 & 738 & 41.8 & 903 & 4.56 & -- & 7.5 & 2620 & 36.55 & 38.68 \\
\hline San & Ysidro - Jemez Pu & blo Area & & & & & & & & & & & & & & & & & \\
\hline 36 & Ponderosa Spring & VA-39 & 11 & 16 & 49 & 10.3 & 0.95 & 447 & 34 & 2.2 & 848 & 193 & 267 & 7.2 & -- & 3.16 & 1880 & 23.93 & 25.83 \\
\hline 36 & Ponderosa Spring & VA-135 & 15 & 14.5 & 51 & 9.3 & 0.89 & 480 & 39 & 2.2 & 862 & 202 & 263 & 7.52 & 0.83 & 3.01 & 1940 & 25.51 & 26.15 \\
\hline 37 & Cañon Spring & $V A-40$ & 18 & 47 & 36 & 5.2 & 0.49 & 161 & 7.6 & 0.5 & 367 & 127 & 71 & 9.3 & -- & 0.41 & 833 & 9.50 & 11.15 \\
\hline 55 & $\begin{array}{l}\text { San Ysidro } \\
\text { Warm Spring }\end{array}$ & VA-33 & 27 & 14 & 375 & 128 & 7.70 & 1710 & 75.3 & 5.30 & 1860 & 1165 & 1820 & 3.95 & -- & 8.32 & 7170 & 106.37 & 106.29 \\
\hline 55 & $\begin{array}{l}\text { San Ysidro } \\
\text { Warm Spring }\end{array}$ & $V A-130$ & 22 & 16.3 & 305 & 71.3 & 4.62 & 1720 & 77 & 8.1 & 1740 & 1183 & 1671 & 3.32 & 5.0 & 9.77 & 6810 & 99.08 & 100.46 \\
\hline 55 & $\begin{array}{l}\text { San Ysidro } \\
\text { Warm Spring }\end{array}$ & $V A-148$ & - & 20 & -- & -- & -- & -- & -- & -- & 1961 & 1181 & 1862 & 4.52 & 4.3 & -- & -- & -- & -- \\
\hline 56 & Zia Hot Kell & VA-34 & 56 & 30 & 302 & 90 & 9.00 & 2650 & 66.7 & 6.7 & 1440 & 3740 & 3000 & 2.40 & -- & 6.52 & 12200 & 140.46 & 186.22 \\
\hline 56 & Zia Hot Hell & VA-53 & 54 & 33 & 321 & 61 & 4.75 & 3440 & 77 & 6.0 & 1068 & 3430 & 3210 & 4.51 & 4.20 & 7.41 & 11700 & 173.54 & 179.70 \\
\hline 56 & Zia Hot Nell & VA-67 & 53 & 35 & 320 & 71.5 & 9.55 & 3180 & 64 & 6.3 & 1400 & 3280 & 2930 & 3.8 & 4.2 & 6.60 & 11300 & 162.76 & 174.08 \\
\hline 56 & Zia Hot Hell & VA-74 & 53 & 34 & 354 & 21.8 & 6.87 & 3700 & 54 & 5.52 & 1400 & 4100. & 3210 & 2.67 & 1.1 & 6.9 & 12800 & 182.60 & 198.99 \\
\hline 56 & Zia Hot Well & $V A-125$ & 53.0 & 33 & 364 & 62.8 & 8.7 & 3080 & 63.4 & 5.2 & 1398 & 3338 & 2984 & 2.47 & 3.4 & 7.8 & 11400 & 159.71 & 176.71 \\
\hline 56 & Zia Hot Nell & $V A-149$ & -- & 34 & -- & -- & -- & -- & -- & -- & 1429 & 3350 & 3020 & 2.89 & 3.5 & -- & -- & -- & -- \\
\hline 57 & Unnamed Hell & VA-35 & 21 & -- & -- & -- & -- & - & -- & -- & - & -- & - & -- & -- & -- & - & -- & -- \\
\hline 58 & Salt Spring & $V A-36$ & 15.5 & 1 & 89 & 26.4 & 4.82 & 1950 & 94 & 7.4 & 1870 & 702 & 2380 & 10.8 & -- & 9.2 & 7150 & 94.93 & 112.97 \\
\hline 59 & Log Spring & VA-37 & 28.5 & -- & -- & -- & -- & -- & -- & - & -- & -- & -- & -- & -- & -- & -- & - & - \\
\hline 60 & owl Spring & $V A-38$ & 16 & 22 & 73 & 10.4 & 0.43 & 34 & 2.1 & 0.176 & 311 & 33.8 & 27.8 & 1.4 & -- & 0.12 & 516 & 6.06 & 6.66 \\
\hline
\end{tabular}

\footnotetext{
a Contaminated with drilling mud.
}

b Baca well samples are not corrected for steam loss; see Table B-I for steam fractions and separation pressures. 
TABLE B-III

TRACE ELEMENT ANALYSES FOR WATERS IN THE JEMEZ MOUNTAINS REGION, NEW MEXICO (VALUES IN mg/l)

\begin{tabular}{|c|c|c|c|c|c|c|c|c|c|c|c|c|c|c|c|c|c|c|c|}
\hline $\begin{array}{l}\text { Map } \\
\text { No. }\end{array}$ & $\begin{array}{l}\text { Field } \\
\text { No. }\end{array}$ & $\mathrm{Ag}$ & A1 & As & $\mathrm{Ba}$ & Cd & Co & $\mathrm{Cr}$ & $\mathrm{Cu}$ & $\mathrm{Fe}$ & $\mathrm{Mn}$ & Mo & $\mathrm{NH}_{4}$ & $\mathrm{Ni}$ & $\mathrm{NO}_{3}$ & $\mathrm{~Pb}$ & $\mathrm{PO}_{4}$ & $R b$ & $2 n$ \\
\hline \multicolumn{20}{|c|}{ Surface and Near-Surface Meteoric Waters } \\
\hline 1 Gallery Spring & LA-1 & $<0.03$ & -- & -- & $<0.12$ & $<0.03$ & $<0.06$ & $<0.03$ & $<0.04$ & $<0.04$ & $<0.02$ & $<0.10$ & -- & $<0.05$ & -- & $<0.14$ & -- & -- & $<0,01$ \\
\hline $2 \mathrm{~T}-3 \mathrm{Kell}$ & LA-2 & $<0.03$ & -- & -- & $<0.12$ & $<0.03$ & $<0.06$ & $<0.03$ & $<0.04$ & 0.53 & 0.11 & $<0.10$ & -- & $<0.05$ & -- & $<0.14$ & -- & -- & 0.01 \\
\hline $3 \mathrm{~T}-2 \mathrm{Kell}$ & LA-3 & $<0.03$ & -- & -- & $<0.12$ & 0.03 & $<0.06$ & $<0.03$ & $<0.04$ & $<0.04$ & $<0.02$ & $<0.10$ & -- & $<0.05$ & -- & $<0.14$ & -- & -- & 0.18 \\
\hline 4 Sacred Spring & $L A-4$ & 0.06 & -- & -- & $<0.12$ & 0.03 & $<0.06$ & $<0.03$ & $<0.04$ & $<0.04$ & $<0.02$ & $<0.10$ & -- & $<0.05$ & -- & $<0.14$ & -- & -- & 0.02 \\
\hline 5 Basalt Spring & LA-5 & $<0.03$ & -- & -- & $<0.12$ & $<0.03$ & $<0.06$ & $<0.03$ & $<0.04$ & $<0.04$ & $<0.02$ & $<0.10$ & -- & $<0.05$ & -- & $<0.14$ & -- & -- & $<0.1$ \\
\hline $6 \mathrm{~L}-6 \mathrm{He} 11$ & $L A-6$ & $<0.03$ & - & -- & $<0.12$ & $<0.03$ & $<0.06$ & $<0.03$ & $<0.04$ & $<0.04$ & $<0.02$ & $<0.10$ & -- & $<0.05$ & -- & $<0.14$ & -- & -- & $<0.01$ \\
\hline 7 L-18 Kell & LA-7 & $<0.03$ & -- & -- & $<0.12$ & $<0.03$ & $<0.06$ & $<0.03$ & $<0.04$ & $<0.04$ & $<0.02$ & $<0.10$ & -- & $<0.05$ & -- & $<0.14$ & -- & -- & $<0.01$ \\
\hline 8 L-5 We 11 & LA-8 & $<0.05$ & -- & -- & $<0.12$ & $<0.03$ & $<0.06$ & $<0.03$ & $<0.04$ & $<0.04$ & $<0.02$ & $<0.10$ & -- & $<0.05$ & -- & $<0.14$ & -- & -- & $<0.01$ \\
\hline 9 L-4 Well & LA-9 & $<0.03$ & -- & -- & $<0.12$ & $<0.03$ & $<0.06$ & $<0.03$ & $<0.04$ & $<0.04$ & $<0.02$ & $<0.10$ & - & $<0.05$ & -- & $<0.14$ & -- & -- & $<0.01$ \\
\hline 10 PM-2 Well & $L A-10$ & $<0.03$ & -- & -- & $<0.12$ & $<0.03$ & $<0.06$ & $<0.03$ & $<0.04$ & $<0.04$ & $<0.02$ & $<0.10$ & -- & $<0.05$ & -- & $<0.14$ & -- & -- & $<0.01$ \\
\hline 11 PM-1 Well & $\lfloor A-11$ & $<0.03$ & -- & $-\infty$ & $<0.12$ & $<0.03$ & $<0.06$ & $<0.03$ & $<0.04$ & $<0.04$ & $<0.02$ & $<0.10$ & -- & $<0.05$ & -- & $<0.14$ & -- & -- & $<0.01$ \\
\hline $12 \mathrm{G}-6 \mathrm{We} 11$ & LA-12 & $<0.03$ & -- & -- & $<0.12$ & $<0.03$ & $<0.06$ & $<0.03$ & $<0.04$ & $<0.04$ & $<0.02$ & $<0.10$ & -- & $<0.05$ & -- & $<0.14$ & -- & -- & $<0.01$ \\
\hline $13 \mathrm{G}-5 \mathrm{Well}$ & LA-13 & $<0.03$ & -- & -- & $<0.12$ & $<0.03$ & $<0.06$ & $<0.03$ & $<0.04$ & $<0.04$ & $<0.02$ & $<0.10$ & -- & $<0.05$ & -- & $<0.14$ & -- & -- & $<0.01$ \\
\hline 14 G-4 Well & LA-14 & $<0.03$ & -- & -- & $<0.12$ & $<0.03$ & $<0.06$ & $<0.03$ & $<0.04$ & $<0.04$ & $<0.02$ & $<0.10$ & -- & $<0.05$ & -- & $<0.14$ & -- & -- & $<0.01$ \\
\hline 15 G-3 Well & LA-15 & $<0.03$ & -- & -- & $<0.12$ & $<0.03$ & $<0.06$ & $<0.03$ & $<0.04$ & $<0.04$ & $<0.02$ & $<0.10$ & -- & $<0.05$ & -- & $<0.14$ & -- & -- & $<0.01$ \\
\hline 16 G-2 Nell & LA-16 & $<0.03$ & -- & -- & $<0.12$ & $<0.03$ & $<0.06$ & $<0.03$ & $<0.04$ & $<0.04$ & $<0.02$ & $<0.10$ & -- & $<0.05$ & -- & $<0.14$ & -- & -- & $<0.01$ \\
\hline 17 G-1A Well & {$[A-17$} & $<0.03$ & -- & -- & $<0.12$ & $<0.03$ & $<0.06$ & $<0.03$ & $<0.04$ & $<0.04$ & $<0.02$ & $<0.10$ & -- & $<0.05$ & -- & $<0.14$ & -- & -- & $<0.01$ \\
\hline $18 \mathrm{G}-1 \mathrm{Kell}$ & $\mathrm{LA}-18$ & 0.06 & -- & -- & $<0.12$ & $<0.03$ & $<0.06$ & $<0.03$ & $<0.04$ & $<0.04$ & $<0.02$ & $<0.10$ & - & $<0.05$ & -- & $<0.14$ & -- & -- & $<0.01$ \\
\hline $\begin{array}{l}19 \text { Spring, White } \\
\text { Rock Canyon }\end{array}$ & LA-19 & $<0.03$ & -- & -- & $<0.12$ & $<0.03$ & $<0.06$ & $<0.03$ & $<0.04$ & $<0.04$ & $<0.02$ & $<0.10$ & -- & $<0.05$ & -- & $<0.14$ & -- & -- & $<0.01$ \\
\hline 20 PM-3 Well & $L A-20$ & 0.06 & -- & -- & $<0.12$ & $<0.03$ & $<0.06$ & $<0.03$ & $<0.04$ & $<0.04$ & $<0.02$ & $<0.10$ & -- & $<0.05$ & -- & $<0.14$ & -- & -- & 0.02 \\
\hline 32 Pajarito Spring & $V A-29$ & $<0.03$ & -- & -- & 0.010 & $<0.03$ & $<0.06$ & 0.018 & $<0.04$ & 0.044 & 0.002 & $<0.10$ & -- & $<0.05$ & -- & $<0.14$ & -- & -- & 0.001 \\
\hline $\begin{array}{l}33 \text { Springs, white } \\
\text { Rock Canyon }\end{array}$ & $V A-30$ & $<0.03$ & -- & -- & 0.14 & $<0.03$ & $<0.06$ & 0.006 & 0.004 & 0.140 & 0.009 & $<0.10$ & - & 0.004 & -- & $<0.14$ & -- & -- & .003 \\
\hline
\end{tabular}


TABLE B-III (cont)

\begin{tabular}{|c|c|c|c|c|c|c|c|c|c|c|c|c|c|c|c|c|c|c|c|c|}
\hline $\begin{array}{l}\text { Map } \\
\text { No. }\end{array}$ & Name & $\begin{array}{l}\text { Field } \\
\text { No. }\end{array}$ & $\mathrm{Ag}$ & A1 & As & $\mathrm{Ba}$ & $\mathrm{Cd}$ & Co & $\mathrm{Cr}$ & $\mathrm{Cu}$ & $\mathrm{Fe}$ & Mn & Mo & $\mathrm{NH}_{4}$ & $\mathrm{Ni}$ & $\mathrm{NO}_{3}$ & $\mathrm{~Pb}$ & $\mathrm{PO}_{4}$ & RD & $\mathrm{Zn}$ \\
\hline 52 & $\begin{array}{l}\text { Unnamed cold } \\
\text { Spring }\end{array}$ & VA-58 & $<0.03$ & - & - & 0.016 & $<0.001$ & $<0.001$ & $<0.001$ & $<0.001$ & 0.15 & 0.002 & $<0.002$ & -- & $<0.002$ & $<0.2$ & $<0.004$ & $<0.2$ & -- & 0.024 \\
\hline 53 & $\begin{array}{l}\text { Unnamed Cold } \\
\text { Spring }\end{array}$ & YA-59 & $<0.03$ & - & -- & 0.022 & $<0.001$ & $<0.001$ & 0.004 & $<0.001$ & 1.19 & 0.135 & $<0.002$ & -- & $<0.002$ & 0.7 & $<0.004$ & $<0.2$ & -- & 0.008 \\
\hline 27 & San Antonio Creek & VA-104 & -- & - & -- & -- & - & -- & -- & -- & -- & -- & - & -- & -- & $<0.10$ & -- & $<0.1$ & -- & -- \\
\hline 28 & San Antonio Creek & VA-24 & -- & - & -- & -- & -- & -- & -- & -- & -- & -- & - & -- & -- & -- & -- & - & -- & - \\
\hline 28 & San Antonio Creek & VA-95 & $<0.01$ & - & -- & 0.07 & $<0.01$ & $<0.01$ & 0.05 & 0.01 & 0.70 & 0.04 & $<0.01$ & $\rightarrow$ & $<0.01$ & 0.69 & $<0.02$ & $<0.02$ & -- & 0.01 \\
\hline 28 & San Antonio Creek & YA-127 & -- & - & -- & 0.03 & -- & -- & -- & -- & -- & -- & -- & $-{ }^{\prime}$ & -- & -- & -- & -- & -- & 0.02 \\
\hline 29 & $\begin{array}{l}\text { Jemez River at } \\
\text { Soda Dam }\end{array}$ & VA-52 & $<0.03$ & -- & -- & 0.021 & $<0.001$ & $<0.001$ & 0.001 & 0.016 & -- & -- & $<0.002$ & - & $\begin{array}{l}<0.004 \\
.003\end{array}$ & $<0.1$ & $\therefore$ & $<0.2$ & - & 0.036 \\
\hline 29 & $\begin{array}{l}\text { Jemez River at } \\
\text { Soda Dam }\end{array}$ & $V A-88$ & -- & -- & -- & -- & -- & -- & -- & -- & - & -- & -- & $\cdots$ & -- & 0.32 & -- & $<0.02$ & -- & -- \\
\hline 29 & $\begin{array}{l}\text { Jemez River at } \\
\text { Soda Dam }\end{array}$ & $V A-111$ & -- & -- & $-\infty$ & $\cdots$ & - & -- & $\cdots$ & -- & -- & -- & -- & -- & -- & $\overline{v i j}$ & -- & -- & $\cdots$ & -- \\
\hline 29 & $\begin{array}{l}\text { Jemez River at } \\
\text { Soda Dam }\end{array}$ & VA-131 & -- & 0.4 & -- & 0.04 & 0.001 & -- & $\rightarrow$ & -- & 0.3 & 0.02 & -- & - & -- & $\because$ & -- & -- & -- & -- \\
\hline 31 & Panorama Spring & $V A-28$ & 0.002 & 0.0013 & -- & 0.176 & $<0.03$ & $<0.06$ & 0.018 & 0.002 & 0.016 & 0.003 & $<0.10$ & -- & 0.002 & $11=2$ & 0.002 & -- & -- & $<0.01$ \\
\hline 31 & Panorama Spring & VA-86 & -- & - & -- & -- & -- & -- & -- & -- & -- & -- & -- & -- & -- & 6.51 & -- & $<0.02$ & -- & -- \\
\hline 31 & Panorama Spring & $V A-136$ & -- & - & -- & 0.15 & -- & -- & -- & -- & -- & -- & $\therefore$ & -- & -- & 0.58 & -- & -- & -- & -- \\
\hline 35 & Sino Spring & VA-32 & - & -- & -- & -- & -- & -- & -- & -- & -- & -- & -- & -- & -- & -- & -- & -- & -- & -- \\
\hline 35 & Sino Spring & VA-63 & $<0.001$ & 0.001 & -- & 0.017 & $<0.001$ & $<0.002$ & $<0.001$ & $<0.001$ & $<0.001$ & $<0.001$ & $<0.002$ & -- & $<0.001$ & $<0.4$ & $<0.004$ & $<0.4$ & -- & 0.007 \\
\hline 35 & Sino Spring & VA-69 & -- & 0.001 & -- & -- & -- & -- & -- & -- & $<0.01$ & 0.01 & - & -- & -- & 1.2 & -- & $<0.1$ & -- & - \\
\hline 35 & Sino Spring & VA-85 & - & -- & -- & -- & -- & -- & -- & -- & -- & -- & -- & -- & -- & 1.06 & -- & $<0.02$ & -- & -- \\
\hline 35 & Sino Spring & $V A-102$ & -- & -- & -- & -- & -- & -- & - & -- & -- & -- & -- & -- & -- & 0.14 & -- & $<0.1$ & -- & -- \\
\hline 38 & $\begin{array}{l}\text { Indian Valley } \\
\text { Well }\end{array}$ & VA-41 & $<0.03$ & -- & -- & 0.053 & $<0.03$ & 0.001 & 0.002 & $<0.04$ & 0.42 & 0.024 & 0.002 & -- & $<0.05$ & -- & $<0.14$ & -- & -- & 0.002 \\
\hline 40 & $\begin{array}{l}\text { Unnamed cold } \\
\text { Spring }\end{array}$ & VA-43 & $<0.03$ & -- & -- & 0.031 & $<0.001$ & $<0.001$ & $<0.001$ & 0.020 & 0.014 & 0.006 & $<0.002$ & -- & $<0.002$ & $<0.2$ & $<0.004$ & $<0.2$ & -- & 0.060 \\
\hline
\end{tabular}




\begin{tabular}{|c|c|c|c|c|c|c|c|c|c|c|c|c|c|c|c|c|c|c|c|c|}
\hline $\begin{array}{l}\text { Map } \\
\text { No. }\end{array}$ & Name & $\begin{array}{l}\text { Field } \\
\text { No. }\end{array}$ & $\mathrm{Ag}$ & A) & As & $\mathrm{Ba}$ & Cd & Co & $\mathrm{Cr}$ & $\mathrm{Cu}$ & $\mathrm{Fe}$ & Mn & Mo & $\mathrm{NH}_{4}$ & $\mathrm{Ni}$ & $\mathrm{NO}_{3}$ & $\mathrm{~Pb}$ & $\mathrm{PO}_{4}$ & $\mathrm{Rb}$ & $\mathrm{Zn}$ \\
\hline & $\begin{array}{l}\text { Unnamed cold } \\
\text { Spring }\end{array}$ & $V A-139$ & -- & 0.2 & - & 0.02 & 0.002 & -- & -- & -- & 0.2 & - & -- & -- & 0.002 & - & $<0.14$ & -- & -- & -- \\
\hline $41 !$ & $\begin{array}{l}\text { Unnamed cold } \\
\text { Spring }\end{array}$ & $V A-44$ & $<0.03$ & -- & -- & 0.129 & $<0.03$ & $<0.06$ & 0.001 & $<0.04$ & 0.025 & 0.002 & $<0.10$ & -- & $<0.05$ & -- & -- & -- & -- & -- \\
\hline & $\begin{array}{l}\text { Unnamed cold } \\
\text { Spring }\end{array}$ & $V A-46$ & $<0.03$ & -- & -- & 0.047 & $<0.03$ & $<0.06$ & 0.001 & $<0.04$ & 0.004 & $<0.001$ & 0.001 & -- & $<0.05$ & -- & $<0.14$ & -- & -- & 0.008 \\
\hline 44 & $\begin{array}{l}\text { Seven Springs } \\
\text { Spring }\end{array}$ & VA-47 & $<0.03$ & -- & -- & 0.036 & $<0.03$ & $<0.06$ & 0.001 & $<0.04$ & 0.036 & 0.001 & 0.001 & -- & $<0.05$ & -- & $<0.14$ & -- & -- & $<0.01$ \\
\hline $44 s$ & $\begin{array}{l}\text { Seven Springs } \\
\text { Spring }\end{array}$ & VA-138 & -- & 0.2 & -- & 0.02 & 0.003 & -- & -- & -- & 0.2 & $-\infty$ & -- & -- & -- & -- & -- & -- & -- & -- \\
\hline 44 & $\begin{array}{l}\text { Cold Spring west } \\
\text { of caldera }\end{array}$ & $V A-134$ & -- & 0.2 & -- & 0.02 & 0.003 & -- & -- & $-\infty$ & 0.2 & -- & -- & -- & -- & 0.19 & -- & -- & -- & - \\
\hline 45 & $\begin{array}{l}\text { Unnamed cold } \\
\text { Spring }\end{array}$ & VA-48 & $<0.03$ & -- & -- & 0.068 & $<0.001$ & $<0.001$ & 0.001 & 0.007 & 0.036 & $<0.001$ & $<0.002$ & -- & $<0.002$ & 2.6 & $<0.004$ & $<0.5$ & -- & 0.300 \\
\hline 46 & Eddy's Hell & YA-49 & $<0.03$ & -- & -- & 0.113 & $<0.001$ & $<0.001$ & $<0.001$ & 0.021 & 0.029 & $<0.001$ & 0.117 & -- & $<0.002$ & $<0.2$ & $<0.004$ & $<0.2$ & -- & 0.040 \\
\hline 46 & Henson's Well & VA-61 & $<0.001$ & -- & -- & 0.134 & $<0.001$ & $<0.002$ & $<0.001$ & 0.088 & $<0.02$ & 0.003 & 0.015 & -- & $<0.001$ & 9.1 & $<0.004$ & $<0.4$ & -- & 0.015 \\
\hline 48 & $\begin{array}{l}\text { Unnamed cold } \\
\text { Spring }\end{array}$ & $V A-54$ & $<0.03$ & -- & -- & 0.065 & $<0.001$ & $<0.001$ & 0.002 & $<0.001$ & 0.360 & 0.009 & $<0.002$ & -- & $<0.002$ & 1.1 & $<0.004$ & $<0.5$ & -- & 0.007 \\
\hline 49 & $\begin{array}{l}\text { Unnamed cold } \\
\text { Spring }\end{array}$ & VA-55 & $<0.03$ & -- & -- & 0.074 & $<0.001$ & $<0.001$ & 0.005 & $<0.001$ & 1.18 & 0.012 & $<0.002$ & -- & $<0.002$ & 0.9 & $<0.004$ & $<0.5$ & -- & 0.015 \\
\hline $50 !$ & $\begin{array}{l}\text { Unnamed cold } \\
\text { Spring }\end{array}$ & VA-56 & $<0.03$ & -- & -- & 0.018 & $<0.001$ & $<0.001$ & 0.002 & $<0.001$ & 0.60 & 0.007 & $<0.002$ & -- & $<0.002$ & $<0.2$ & $<0.004$ & $<0.5$ & -- & 0.018 \\
\hline 51 & Apache Spring & VA-57 & $<0.03$ & -- & -- & 0.066 & $<0.001$ & $<0.001$ & 0.002 & $<0.001$ & 0.42 & 0.003 & $<0.002$ & -- & $<0.002$ & 1.4 & $<0.004$ & $<0.2$ & -- & 0.020 \\
\hline 54 & Turkey Spring & $V A-60$ & $<0.03$ & - & -- & 0.056 & $<0.001$ & $<0.001$ & $<0.001$ & $<0.001$ & $0: 012$ & 0.001 & $<0.002$ & -- & $<0.002$ & 1.3 & $<0.004$ & $<0.2$ & - & 0.080 \\
\hline 54 & Turkey Spring & VA-137 & -- & -- & -- & 0.02 & 0.002 & -- & -- & -- & -- & -- & -- & -- & -- & 1.57 & -- & - & -- & -- \\
\hline 681 & Las Conchas Spring & $V A-124$ & -- & 0.05 & -- & 0.01 & - & -- & -- & -- & 0.02 & -- & - & -- & -- & -- & -- & -- & - & -- \\
\hline
\end{tabular}


TABLE B-III (cont)

\begin{tabular}{|c|c|c|c|c|c|c|c|c|c|c|c|c|c|c|c|c|c|c|c|}
\hline $\begin{array}{l}\text { Map } \\
\text { No. }\end{array}$ & $\begin{array}{l}\text { Field } \\
\text { No. }\end{array}$ & Ag & A) & As & $\mathrm{Ba}$ & $\mathrm{Cd}$ & Co & $\mathrm{Cr}$ & $\mathrm{Cu}$ & $\mathrm{Fe}$ & Mn & Mo & $\mathrm{NH}_{4}$ & Ni & $\mathrm{NO}_{3}$ & $\mathrm{~Pb}$ & $\mathrm{PO}_{4}$ & $R D$ & $2 n$ \\
\hline Sulphur Springs Area & & & & & & & & & & & & & & & & & & & \\
\hline $\begin{array}{l}21 \text { Mudpot, Men's } \\
\text { Bathhouse }\end{array}$ & VA-13 & $<0.03$ & -- & -- & $<0.12$ & $<0.03$ & $<0.06$ & $<0.03$ & 0.63 & 13 & 0.01 & $<0.10$ & -- & $<0.05$ & -- & $<0.14$ & -- & -- & 0.16 \\
\hline $\begin{array}{l}21 \text { Mudpot, Men's } \\
\text { Bathhouse }\end{array}$ & $S-7-80$ & $<0.03$ & -- & -- & 0.083 & $<0.001$ & 0.059 & -- & 0.030 & 37 & 0.64 & 0.010 & -- & -- & -- & -- & -- & -- & -- \\
\hline $\begin{array}{l}21 \text { Mudpot, Men's } \\
\text { Ba thhouse }\end{array}$ & VA-75 & -- & -- & -- & -- & -- & -- & -- & -. & -- & -- & -- & -- & -- & -- & -- & -- & -- & -- \\
\hline $\begin{array}{l}21 \text { Steam, Men's } \\
\text { Ba thhouse }\end{array}$ & $V A-81$ & $\therefore$ & -- & -- & - & -- & -- & -- & -- & -- & -- & -- & -- & -- & $<0.02$ & -- & $<0.02$ & -- & -- \\
\hline 21 Women's Bathhouse & $S-6-80$ & $<0.03$ & -- & -- & 0.053 & 0.001 & 0.245 & -- & -- & 490 & 8.10 & 0.10 & -- & -- & -- & -- & - & -- & -- \\
\hline 21 Women's Bathhouse & VA-76 & $\rightarrow$ & -- & -- & -- & -- & -- & -- & -- & -- & -- & -- & -- & - & -- & -- & -- & -- & -- \\
\hline 21 Footbath Springs & $S-4-80$ & $<0.03$ & -- & -- & 0.030 & 0.004 & 0.460 & -- & -- & 468 & 4.65 & 0.005 & -- & -- & -- & -- & -- & -- & -- \\
\hline 21 Footbath Springs & VA-77 & -- & -- & -- & -- & -- & -- & -- & -- & -- & -- & -- & -- & -- & -- & -- & -- & -- & -- \\
\hline 21 Footbath Springs & VA-79 & -- & -- & -- & -- & -- & -- & -- & -- & -- & -- & -- & -- & -- & - & -- & -- & -- & -- \\
\hline 21 Unnamed Spring & $5-3-80$ & $<0.03$ & -- & -- & 0.010 & $<0.001$ & 0.024 & 0.052 & $<0.002$ & 16.2 & 2.84 & $<0.002$ & -- & 0.039 & -- & 0.032 & -- & -- & - \\
\hline $\begin{array}{l}21 \text { Unnamed Hot } \\
\text { Spring. }\end{array}$ & $S-9-80$ & $<0.03$ & -- & -- & 0.037 & $<0.001$ & 0.017 & 0.063 & -- & 38 & 3.20 & $<0.002$ & -- & 0.042 & -- & 0.120 & -- & -- & -- \\
\hline $\begin{array}{l}21 \text { Unnamed Hot } \\
\text { Spring }\end{array}$ & $V A-14$ & $<0.03$ & 3.17 & -- & 0.012 & 0.03 & 0.07 & 0.06 & $<0.04$ & 18.1 & 11.7 & $<0.10$ & - & $<0.05$ & -- & $<0.14$ & -- & -- & 0.08 \\
\hline 21 Electric Spring & $S-5-80$ & $<0.03$ & - & -- & 0.035 & $<0.001$ & 0.100 & 0.360 & 0.080 & 127 & 2.40 & 0.004 & -- & 0.220 & -- & 0.080 & - & -- & 0.640 \\
\hline 21 Lemonade Spring & $S-10-80$ & $<0.03$ & - & -- & 0.055 & $<0.001$ & 0.010 & 0.056 & $<0.001$ & 38 & 3.76 & $<0.002$ & -- & 0.016 & -- & 0.004 & -- & -- & -- \\
\hline 21 Sulphur creek & VA-78 & -- & - & -- & -- & -- & -- & - & -- & -- & -- & - & -- & - & -- & -- & -- & -- & -- \\
\hline $\begin{array}{l}21 \text { Steam, Main } \\
\text { Fumarole }\end{array}$ & VA-80 & -- & -- & -- & -- & -- & -- & -- & -- & -- & -- & -- & 2.57 & - & $<0.02$ & -- & $<0.02$ & -- & -- \\
\hline $\begin{array}{l}22 \text { Spring. Alamo } \\
\text { Canyon }\end{array}$ & $S-1-80$ & $<0.03$ & -- & -- & 0.050 & $<0.001$ & $<0.001$ & 0.019 & 0.001 & 16.3 & 0.44 & $<0.002$ & -- & 0.008 & -- & 0.004 & -- & -- & 0.880 \\
\hline $\begin{array}{l}22 \text { Creek, Alamo } \\
\text { Canyon }\end{array}$ & $5-2-80$ & $<0.03$ & - & -- & 0.42 & $<0.001$ & 0.002 & 0.005 & 0.008 & 1.1 & 0.35 & $<0.002$ & -- & 0.003 & -- & 0.004 & -- & -- & 0.560 \\
\hline 22 Bubbling Seep & VA-23 & $<0.03$ & - & -- & $<0.12$ & $<0.03$ & $<0.06$ & $<0.03$ & $<0.04$ & 0.28 & 0.96 & $<0.10$ & - & $<0.05$ & -- & $<0.14$ & - & -- & $<0.01$ \\
\hline
\end{tabular}




\begin{tabular}{|c|c|c|c|c|c|c|c|c|c|c|c|c|c|c|c|c|c|c|c|c|}
\hline $\begin{array}{l}\text { Map } \\
\text { No. }\end{array}$ & Name & $\begin{array}{l}\text { Field } \\
\text { No. }\end{array}$ & $\mathrm{Ag}$ & A1 & As & $\mathrm{Ba}$ & cd & co & $\mathrm{Cr}$ & $\mathrm{Cu}$ & $\mathrm{Fe}$ & $M n$ & Mo & $\mathrm{NH}_{4}$ & $\mathbf{N i}$ & $\mathrm{NO}_{3}$ & $\mathrm{~Pb}$ & $\mathrm{PO}_{4}$ & RD & $\mathrm{Zn}$ \\
\hline 23 & Bubbling Pool & VA-22 & $<0.03$ & -- & -- & $<0.12$ & $<0.03$ & $<0.06$ & $<0.03$ & $<0.04$ & 1.37 & 0.30 & $<0.10$ & -- & $<0.05$ & -- & $<0.14$ & - & -- & $<0.01$ \\
\hline 24 & $\begin{array}{l}\text { Spring, Short } \\
\text { Canyon }\end{array}$ & $5-8-80$ & $<0.03$ & -- & -- & 0.109 & $<0.001$ & 0.005 & 0.010 & 0.007 & 5.5 & 0.54 & $<0.002$ & -- & 0.001 & -- & $<0.004$ & - & -- & .160 \\
\hline 63 & $\begin{array}{l}\text { GRI Mudpit at } \\
\text { WC } 23-4\end{array}$ & VA-107 & -- & 0.52 & 0.3 & 0.05 & 0.004 & -- & 0.003 & 0.030 & 0.25 & 0.02 & -- & -- & 0.013 & -- & -- & -- & -- & 0.12 \\
\hline 63 & $\begin{array}{l}\text { GRI Well at } \\
4800 \mathrm{ft}\end{array}$ & VA-113 & -- & 0.79 & 4.1 & 1.35 & - & 0.015 & 0.01 & -- & 3.15 & 1.35 & -- & -- & 0.025 & -- & 0.016 & -- & - & .04 \\
\hline 63 & $\begin{array}{l}\text { GRI Hell at } \\
4800 \mathrm{ft}\end{array}$ & VA-114 & - & -- & 4.42 & 1.61 & -- & -- & - & 0.912 & 12.5 & 2.99 & 0.114 & - & 0.035 & -- & 1.32 & -- & 7.8 & .29 \\
\hline 63 & $\begin{array}{l}\text { GRI Well at } \\
6300 \mathrm{ft}\end{array}$ & $V A-116$ & - & 0.54 & 7.8 & 1.17 & - & 0.019 & 0.01 & -- & 0.26 & 1.08 & - & -- & 0.017 & -- & 0.05 & -- & -- & 3.42 \\
\hline & Iles Caldera - R & & Zone & & & & & & & & & & & & & & & & & \\
\hline 25 & Spence Hot Spring & VA-1 & $<0.03$ & - & -- & $<0.12$ & $<0.03$ & $<0.06$ & $<0.03$ & $<0.04$ & $<0.04$ & $<0.02$ & $<0.10$ & $-\rightarrow$ & $<0.05$ & -- & $<0.14$ & -- & -- & 0.02 \\
\hline 25 & Spence Hot Spring & $V A-68$ & $<0.03$ & 0.065 & -- & $<0.12$ & $<0.03$ & $<0.06$ & $<0.03$ & $<0.04$ & $<0.01$ & $<0.01$ & $<0.10$ & -- & $<0.05$ & $<0.1$ & $<0.14$ & $<0.1$ & -- & $<0.01$ \\
\hline 25 & Spence Hot Spring & VA-72 & - & 0.030 & -- & -- & -- & -- & -- & -- & $<0.01$ & $<0.01$ & -- & 0.03 & -- & $<0.02$ & -- & $<0.02$ & -- & -- \\
\hline 25 & Spence Hot Spring & VA-83 & - & -- & -- & -- & -- & -- & -- & -- & -- & -- & -- & - & -- & $<0.02$ & -- & $<0.02$ & -- & -- \\
\hline 25 & Spence Hot Spring & $V A-105$ & - & -- & - & -- & -- & -- & - & -- & -- & -- & - & -- & -- & 0.13 & -- & $<0.1$ & -- & -- \\
\hline 25 & Spence Hot Spring & $V A-120$ & - & 0.11 & -- & -- & -- & -- & -- & - & -- & -- & -- & - & -- & 0.2 & -- & -- & -- & -- \\
\hline 25 & $\begin{array}{l}\text { Little Spence Hot } \\
\text { Spring }\end{array}$ & VA-2 & $<0.03$ & -- & - & $<0.12$ & $<0.03$ & $<0.06$ & $<0.03$ & $<0.04$ & $<0.04$ & $<0.02$ & 0.10 & - & $<0.05$ & -- & $<0.14$ & -- & -- & 0.02 \\
\hline 26 & McCauley Spring & $V A-3$ & $<0.03$ & -- & -- & $<0.12$ & $<0.03$ & $<0.06$ & $<0.03$ & $<0.04$ & $<0.04$ & $<0.02$ & $<0.10$ & - & $<0.05$ & - & $<0.14$ & - & -- & $<0.01$ \\
\hline 26 & McCauley Spring & VA-87 & -- & -- & -- & -- & -- & - & -- & -- & -- & -- & -- & -- & -- & 1.67 & - & $<0.02$ & -- & -- \\
\hline 26 & McCauley Spring & VA-119 & - & 0.08 & - & -- & -- & - & -- & -- & -- & -- & -- & - & - & 1.30 & -- & - & - & -- \\
\hline 27 & $\begin{array}{l}\text { San Antonio Hot } \\
\text { Spring }\end{array}$ & VA-4 & $<0.03$ & -- & -- & $<0.12$ & $<0.03$ & $<0: 06$ & $<0.03$ & $<0.04$ & $<0.04$ & 0.02 & $<0.10$ & - & $<0.05$ & -- & $<0.14$ & - & -- & $<0.01$ \\
\hline 27 & $\begin{array}{l}\text { San Antonio Hot } \\
\text { Spring }\end{array}$ & $V A-96$ & $<0.01$ & -- & -- & $<0.01$ & $<0.01$ & $<0.01$ & 0.06 & 0.01 & 0.02 & $<0.01$ & $<0.01$ & -- & $<0.01$ & 1.77 & $<0.02$ & $<0.02$ & -- & $<0.01$ \\
\hline 27 & $\begin{array}{l}\text { San Antonio Hot } \\
\text { Spring }\end{array}$ & $V A-128$ & -- & -- & -- & 0.01 & -- & -- & -- & -- & -- & -- & -- & - & -- & 2.2 & -- & -- & -- & 0.02 \\
\hline & thhouse Spring & -20 & .03 & .041 & -- & 0.12 & 0.03 & .06 & $<0.03$ & 0.04 & $<0.08$ & 0.04 & 0.10 & - & $<0.05$ & -- & $<0.14$ & -- & -- & 0.01 \\
\hline
\end{tabular}


TABLE B-III (cont)

\begin{tabular}{|c|c|c|c|c|c|c|c|c|c|c|c|c|c|c|c|c|c|c|c|c|}
\hline $\begin{array}{l}\text { Map } \\
\text { No. }\end{array}$ & Name & $\begin{array}{c}\text { Field } \\
\text { No. }\end{array}$ & $\mathrm{Ag}$ & A) & As & $\mathrm{Ba}$ & $\mathrm{Cd}$ & Co & $\mathrm{Cr}$ & $\mathrm{Cu}$ & Fe & Mn & Mo & $\mathrm{NH}_{4}$ & $\mathrm{Ni}$ & $\mathrm{NO}_{3}$ & $\mathrm{~Pb}$ & $\mathrm{PO}_{4}$ & $\mathbf{R b}$ & Zn \\
\hline 28 & Bathhouse Spring & $V A-94$ & $<0.01$ & -- & -- & 0.02 & $<0.01$ & $<0.01$ & 0.05 & 0.01 & 0.01 & $<0.01$ & $<0.01$ & -- & $<0.01$ & 1.36 & $<0.02$ & $<0.02$ & -- & 0.03 \\
\hline 28 & Ba thhouse Spring & VA-126 & - & -- & -- & 0.01 & -- & -- & -- & -- & 0.1 & -- & -- & -- & 0.02 & 0.3 & -- & - & -- & 0.02 \\
\hline 34 & Battleship Seep & $V A-31$ & 0.005 & 0.096 & $=-$ & 0.062 & 0.002 & $<0.06$ & $<0.03$ & $<0.04$ & 0.005 & $<0.001$ & 0.002 & -- & $<0.05$ & -- & $<0.14$ & -- & -- & $<0.01$ \\
\hline 34 & Battleship Seep & $V A-50$ & $<0.03$ & -- & $\cdots$ & 0.022 & $<0.001$ & $<0.001$ & $<0.001$ & $<0.001$ & 0.004 & $<0.001$ & $<0.002$ & -- & $<0.002$ & $<0.1$ & 0.016 & $<0.2$ & -- & $<0.002$ \\
\hline 34 & Battleship Seep & VA-133 & $\cdots$ & -- & -- & 0.04 & -- & -- & -- & -- & -- & - & 0.05 & -- & $\cdots$ & - & -- & $\cdots$ & -- & - \\
\hline 34 & $\begin{array}{l}\text { East Fork, Jemez } \\
\text { River }\end{array}$ & $V A-84$ & -- & - & -- & -- & -- & -- & -- & -- & -- & - & -- & -- & -- & 0.34 & - & $<0.02$ & -- & -- \\
\hline 34 & East Fork, Jemez & VA-106 & - & - & $-\therefore$ & - & -- & -- & -- & -- & - & -- & -- & -- & - & $<0.10$ & -- & $<0.1$ & -- & -- \\
\hline 39 & $\begin{array}{l}\text { Valle Grande } \\
\text { Spring }\end{array}$ & $V A-42$ & $<0.03$ & -- & -- & 0.010 & $<0.001$ & $<0.001$ & 0.002 & $<0.002$ & 0.006 & $<0.001$ & $<0.002$ & -- & $<0.002$ & 1.9 & $<0.004$ & $<0.2$ & -- & -- \\
\hline 39 & $\begin{array}{l}\text { Valle Grande } \\
\text { Spring }\end{array}$ & YA-82 & - & -- & -- & -- & -- & -- & -- & -- & -- & -- & -- & -- & -- & 1.06 & -- & $<0.02$ & -- & -- \\
\hline 39 & $\begin{array}{l}\text { Yalle Grande } \\
\text { Spring }\end{array}$ & VA-117 & -- & - & -- & -- & -- & -- & -- & -- & -- & -- & -- & -- & -- & - & -- & -- & -- & -- \\
\hline 42 & Horseshoe Spring & $V A-45$ & $<0.03$ & -- & -- & 0.119 & $<0.03$ & 0.001 & 0.008 & $<0.04$ & $<0.004$ & $<0.001$ & 0.001 & -- & $<0.05$ & -- & $<0.14$ & -- & -- & $<0.01$ \\
\hline 42 & Horseshoe Spring & VA-118 & - & 0.53 & -- & 0.11 & - & -- & 0.008 & - & 0.02 & $\cdots$ & -- & -- & 0.002 & 0.27 & -- & -- & -- & $<0.02$ \\
\hline 61 & $\mathrm{PC1}$ at $225 \mathrm{ft}$ & $\mathrm{PCl}-7$ & - & -- & -- & 0.12 & - & -- & -- & -- & -- & - & -- & 0.68 & -- & -- & -- & -- & 0.005 & -- \\
\hline 61 & $\mathrm{PCl}$ at $365-391 \mathrm{ft}$ & PCl-8 & -- & -- & -- & 0.07 & -- & -- & 0.003 & -- & -- & -- & - & -- & -- & 1.31 & -- & -- & 0.006 & 0.03 \\
\hline 61 & $\mathrm{PC1}$ at $685-691 \mathrm{ft}$ & PC1-9 & - & -- & -- & 0.18 & -- & -- & -- & -- & -- & -- & -- & -- & -- & 1.42 & -- & -- & -- & -- \\
\hline 61 & $\mathrm{PCl}$ at $943 \mathrm{ft}$ & $P C 1-10$ & -- & -- & -- & 0.14 & - & -- & -- & - & 0.07 & 0.03 & -- & -- & 0.005 & 1.13 & -- & -- & -- & -- \\
\hline 61 & PCl at $1100 \mathrm{ft}$ & PC1-11 & -- & -- & -- & 0.79 & -- & -- & -- & -- & 0.14 & 0.05 & -- & -- & -- & 0.28 & -- & -- & 0.004 & -- \\
\hline 61 & $\mathrm{PCl}$ at $1712 \mathrm{ft}$ & PC1-1 & -- & -- & -- & 0.12 & - & -- & 0.001 & - & 0.16 & 0.28 & -- & -- & 0.050 & -- & -- & - & 0.018 & -- \\
\hline 61 & PC1 at $1937 \mathrm{ft}$ & $\mathrm{PCl}-2$ & -- & 8.6 & -- & 0.04 & -- & -- & 0.050 & -- & 0.30 & 0.02 & -- & -- & 0.24 & -- & -- & -- & 0.012 & -- \\
\hline 61 & PC1 at $1987 \mathrm{ft}$ & $P C 1-3$ & -- & -- & -- & 0.15 & -- & -- & 0.015 & 0.004 & 0.10 & 0.02 & -- & -- & 0.30 & - & -- & -- & 0.008 & -- \\
\hline 61 & PCl at $1953 \mathrm{ft}$ & $\mathrm{PCl}-4$ & - & - & -- & 0.06 & -- & -- & 0.002 & 0.020 & 0.08 & 0.15 & -- & -- & -- & - & -- & -- & 0.005 & -- \\
\hline 61 & $\mathrm{PC1}$ at $2036 \mathrm{ft}$ & $\mathrm{PCl}-5$ & -- & -- & -- & 0.06 & -- & -- & 0.095 & -- & 0.06 & 0.02 & -- & -- & -- & -- & -- & -- & -- & -- \\
\hline
\end{tabular}




\begin{tabular}{|c|c|c|c|c|c|c|c|c|c|c|c|c|c|c|c|c|c|c|c|c|}
\hline $\begin{array}{l}\text { Map } \\
\text { No. }\end{array}$ & Name & $\begin{array}{l}\text { Field } \\
\text { No. }\end{array}$ & $\mathrm{Ag}$ & Al & As & $\mathrm{Ba}$ & $\mathrm{cd}$ & Co & $\mathrm{cr}$ & $\mathrm{Cu}$ & $\mathrm{Fe}$ & $M n$ & Mo & $\mathrm{NH}_{4}$ & $\mathrm{Ni}$ & $\mathrm{NO}_{3}$ & $\mathrm{~Pb}$ & $\mathrm{PO}_{4}$ & $R D$ & $\mathrm{Zn}$ \\
\hline
\end{tabular}

\begin{tabular}{llllllllllllllllllllllllll}
\hline 1 & PC2 at $587-600 \mathrm{ft}$ & PC2-1 & $<0.01$ & 0.1 & $<0.1$ & 0.06 & $0.005<0.001$ & $0.003<0.001$ & 0.06 & $<0.01$ & $<0.2$ & $0.06<0.001$ & $0.03<0.002<0.01<0.001<0.01$
\end{tabular} 71 PC2 at 630-635 ft PC2-2 $<0.01 \quad 0.5<0.1 \quad 0.05<0.001<0.001 \quad 0.001<0.001 \quad 0.19<0.01<0.2 \quad 0.13<0.001 \quad 0.39<0.002<0.01<0.001<0.01$

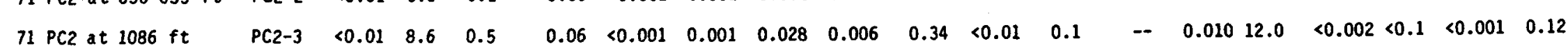
$\begin{array}{lllllllllllllllllll}71 & \text { PC2 at } 1350 \mathrm{ft} & \text { PC2-4 } & <0.01 & 0.1 & 0.6 & 0.47 & <0.001<0.001 & 0.003 & 0.002 & <0.01 & 0.02 & <0.1 & 0.73 & 0.009 & <0.1 & <0.002<0.1 & <0.001<0.01\end{array}$ $\begin{array}{llllllllllllllll}71 & \text { PC2 at } 1360 \mathrm{ft} & \text { PC2-5 } & <0.01<0.1 & 0.5 & 0.65 & <0.001 & 0.003 & 0.005<0.001 & <0.01 & 0.10 & 0.1 & 0.71 & 0.009<0.1 & <0.002<0.1<0.001<0.01\end{array}$

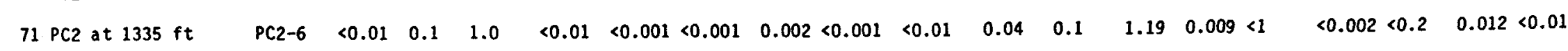
$\begin{array}{llllllllllllllllll}71 \mathrm{PC} 2 \text { at } 1490 \mathrm{ft} & \mathrm{PC} 2-7 & <0.01<0.1 & 0.5 & 0.34 & <0.001<0.001 & 0.001<0.001 & <0.01 & 0.21 & <0.1 & 0.61 & 0.005<0.5 & <0.002<0.1 & 0.008<0.01\end{array}$

\section{Valles Caldera - Baca Geothermal Field}

\begin{tabular}{|c|c|c|c|c|c|c|c|c|c|c|c|c|c|c|c|c|c|c|c|c|}
\hline 62 & $\begin{array}{l}\text { Redondo Creek } \\
\text { at Union Gate }\end{array}$ & VA-129 & -- & 2.1 & -- & 0.04 & 0.001 & -- & -- & -- & 0.9 & 0.01 & -- & -- & -- & -- & -- & -- & -- & $-\rightarrow$ \\
\hline 64 & Baca Well "4 & $B A-2$ & $<0.01$ & 0.36 & 3.1 & 0.02 & $<0.01$ & 0.03 & 0.05 & 0.03 & 0.03 & $<0.01$ & 0.07 & 0.54 & 0.04 & $<0.1$ & 0.02 & $<1$ & 4.1 & $<0.01$ \\
\hline 64 & Baca Well \#4 & BA-5 & $<0.01$ & 0.41 & 2.8 & 0.02 & $<0.01$ & 0.03 & 0.04 & 0.04 & 0.02 & $<0.01$ & 0.05 & 0.17 & 0.05 & $<0.1$ & 0.08 & $<1$ & 2.5 & $<0.01$ \\
\hline 65 & Baca Well 13 & BA-1 & $<0.01$ & 0.40 & 3.3 & 0.02 & $<0.01$ & 0.02 & 0.04 & 0.02 & 0.02 & 0.01 & 0.03 & 0.41 & 0.03 & $<0.1$ & 0.05 & $<1$ & 4.1 & $<0.01$ \\
\hline 65 & Baca Well $: 13$ & $B A-4$ & $<0.01$ & 0.34 & 3.0 & 0.02 & 0.01 & 0.03 & 0.05 & 0.03 & 0.02 & $<0.01$ & 0.04 & 0.21 & 0.04 & $<0.1$ & $<0.01$ & $<1$ & 3.0 & 0.02 \\
\hline 66 & Baca We11 \#15 & BA-7 & $<0.01$ & 0.51 & 3.4 & 0.03 & $<0.01$ & 0.06 & 0.06 & 0.05 & 0.02 & $<0.01$ & 0.24 & 0.87 & 0.07 & $<0.1$ & 0.03 & $<1$ & 4.5 & $<0.01$ \\
\hline 66 & Baca Hell 15 & BA-8 & $<0.01$ & 0.17 & 3.6 & 0.02 & $<0.01$ & 0.01 & 0.01 & 0.02 & 0.01 & $<0.01$ & 0.09 & 0.24 & 0.02 & $<0.1$ & 0.05 & $<1$ & 5.4 & $<0.01$ \\
\hline 66 & Baca Hell 19 & $8 A-9$ & $<0.01$ & 0.37 & 4.1 & 0.02 & $<0.01$ & $<0.01$ & 0.03 & 0.04 & 0.02 & 0.02 & 0.08 & 0.24 & 0.02 & $<0.1$ & 0.09 & $<1$ & 5.0 & $<0.01$ \\
\hline 67 & Baca Well $: 24$ & $B A-3$ & $<0.01$ & 0.46 & 4.1 & 0.014 & 0.01 & 0.03 & 0.04 & 0.03 & 0.01 & $<0.01$ & 0.35 & 0.33 & 0.02 & $<0.1$ & 0.06 & $<1$ & 2.9 & $<0.01$ \\
\hline $\begin{array}{l}67 \\
\text { val }\end{array}$ & $\begin{array}{l}\text { Baca Hell } 24 \\
\text { lles Caldera - Soda }\end{array}$ & $\begin{array}{l}\text { BA-6 } \\
\text { Dam and }\end{array}$ & $<0.01$ & $\begin{array}{l}0.72 \\
\text { prings }\end{array}$ & $\begin{array}{l}3.5 \\
\text { Area } \\
\end{array}$ & 0.013 & $<0.01$ & 0.05 & 0.07 & 0.05 & 0.02 & $<0.01$ & 0.26 & 0.85 & 0.06 & $<0.1$ & $<0.01$ & $<1$ & 2.8 & $<0.01$ \\
\hline 29 & Soda Dam Spring & VA-6 & $<0.03$ & - & -- & 0.12 & $<0.03$ & $<0.06$ & $<0.03$ & $<0.04$ & 0.11 & 0.55 & $<0.10$ & -- & $<0.05$ & -- & $<0.14$ & -- & -- & 0.02 \\
\hline 29 & Soda Dam Spring & VA-9 & $<0.03$ & 0.036 & -- & $<0.12$ & 0.06 & $<0.06$ & $<0.03$ & $<0.04$ & 0.14 & 0.47 & $<0.10$ & -- & 0.05 & -- & $<0.14$ & -- & - & 0.04 \\
\hline 29 & Soda Dam Spring & VA-26 & 0.002 & 0.025 & -- & 0.396 & 0.002 & $<0.06$ & 0.062 & 0.004 & 0.08 & 0.54 & $<0.10$ & -- & $<0.05$ & -- & 0.014 & -- & -- & $<0.01$ \\
\hline 29 & Soda Dam Spring & $V A-51$ & $<0.03$ & - & -- & 0.206 & $<0.001$ & $<0.001$ & $<0.001$ & $<0.001$ & -- & - & $<0.002$ & -- & 0.02 & $<0.1$ & 0.044 & $<0.2$ & -- & $<0.002$ \\
\hline 29 & Soda Dam Spring & $V A-64$ & $<0.001$ & - & -- & 0.430 & $<0.001$ & $<0.002$ & $<0.001$ & $<0.001$ & 0.07 & 0.43 & $<0.002$ & -- & $<0.006$ & $<0.4$ & $<0.004$ & $<0.4$ & -- & $<0.002$ \\
\hline 29 & Soda Dam Spring & $V A-70$ & $<0.03$ & 0.020 & - & $<0.12$ & 0.03 & $<0.06$ & $<0.03$ & $<0.04$ & 0.05 & 0.53 & $<0.10$ & -- & 0.10 & $<0.1$ & $<0.14$ & 8 & - & \\
\hline
\end{tabular}


TABLE B-III (cont)

\begin{tabular}{|c|c|c|c|c|c|c|c|c|c|c|c|c|c|c|c|c|c|c|c|c|}
\hline $\begin{array}{l}\text { Map } \\
\text { No. }\end{array}$ & Name & $\begin{array}{l}\text { Field } \\
\text { No. }\end{array}$ & $\mathrm{Ag}$ & Al & As & $\mathrm{Ba}$ & $\mathrm{cd}$ & co & $\mathrm{Cr}$ & $\mathrm{Cu}$ & $\mathrm{Fe}$ & Mn & Mo & $\mathrm{NH}_{4}$ & $\mathrm{Ni}$ & $\mathrm{NO}_{3}$ & $\mathrm{~Pb}$ & $\mathrm{PO}_{4}$ & $\mathrm{Rb}$ & $\mathrm{Zn}$ \\
\hline 29 & Soda Dam Spring & VA-73 & -- & 0.014 & 1.5 & 0.442 & - & - & -- & -- & 0.08 & 0.68 & -- & 0.55 & -- & $<0.02$ & -- & $<0.02$ & -- & -- \\
\hline 29 & Soda Dam Spring & VA-89 & - & - & 1.6 & -- & -- & -- & -- & -- & -- & -- & -- & -- & -- & $<0.02$ & -- & $<0.02$ & -- & -- \\
\hline 29 & Soda Dam Spring & VA-99 & -- & -- & 1.2 & -- & -- & - & -- & -. & -- & $\cdots$ & -- & -- & -- & -- & -- & -- & -- & -- \\
\hline 29 & Soda Dam Spring & VA-109 & -- & 0.11 & 1.8 & 0.46 & -- & -- & -- & -- & 0.07 & 0.56 & -- & -- & 0.003 & - & -- & -- & -- & 0.01 \\
\hline 29 & Soda Dam Spring & YA-132 & - & -- & 1.5 & 0.41 & - & - & -- & - & 0.1 & 0.51 & -- & $\therefore$ & -- & - & - & - & - & -- \\
\hline 29 & Soda Dam Spring & $V A-140$ & -- & 0.3 & 1.5 & 0.45 & 0.008 & -- & 0.001 & -- & 0.09 & 0.97 & -- & -- & -- & -- & -- & -- & 3.25 & 0.01 \\
\hline 29 & Soda Dam Spring & VA-146 & -- & -- & -- & -- & -- & -- & -- & -- & -- & - & - & -- & -- & - & -- & -- & -- & -- \\
\hline 29 & Grotto Spring & VA-5 & $<0.03$ & -- & -- & $<0.12$ & $<0.03$ & 0.09 & $<0.03$ & $<0.04$ & 0.11 & 0.50 & $<0.10$ & -- & $<0.05$ & -- & $<0.14$ & -- & -- & 0.01 \\
\hline 29 & $\begin{array}{l}\text { Outfall of Soda } \\
\text { Dam Spring }\end{array}$ & VA-65 & $<0.001$ & -- & -- & 0.360 & $<0.001$ & $<0.002$ & $<0.001$ & $<0.001$ & 0.03 & 0.03 & $<0.002$ & -- & $<0.001$ & $<0.4$ & $<0.004$ & $<0.4$ & -- & $<0.002$ \\
\hline 29 & $\begin{array}{l}\text { Hidden Warm } \\
\text { Spring }\end{array}$ & YA-27 & 0.002 & 0.007 & -- & 0.206 & 0.002 & 0.002 & 0.098 & 0.006 & 0.50 & 0.56 & $<0.10$ & -- & $<0.05$ & -- & 0.006 & -- & -- & $<0.001$ \\
\hline 29 & $\begin{array}{l}\text { Hidden Harm } \\
\text { Spring }\end{array}$ & VA-90 & - & -- & 1.2 & -- & -- & -- & -- & -- & -- & -- & -- & - & -- & $<0.02$ & -- & $<0.02$ & -- & -- \\
\hline 29 & $\begin{array}{l}\text { Hidden Warm } \\
\text { Spring }\end{array}$ & $V A-110$ & - & 0.13 & 0.1 & 0.39 & -- & -- & 0.001 & - & 0.51 & 0.50 & - & -- & 0.002 & - & -- & -- & -- & 0.02 \\
\hline 29 & $\begin{array}{l}\text { Hidden Harm } \\
\text { Spring }\end{array}$ & $V A-141$ & -- & - & 1.2 & 0.37 & -- & -- & 0.005 & -- & 0.45 & 0.90 & -- & -- & -- & -- & -- & -- & 2.85 & 0.01 \\
\hline 30 & Main Jemez Spring & $Y A-10$ & $<0.03$ & 0.015 & - & $<0.12$ & 0.03 & $<0.06$ & $<0.03$ & $<0.04$ & 0.20 & 0.17 & $<0.10$ & -- & 0.10 & -- & $<0.14$ & -- & -- & 0.03 \\
\hline 30 & Main Jemez Spring & $V A-18$ & $<0.03$ & 0.014 & -- & 0.24 & $<0.03$ & $<0.06$ & $<0.03$ & $<0.04$ & 0.02 & 0.10 & $<0.10$ & -- & $<0.05$ & - & $<0.14$ & -- & - & $<0.01$ \\
\hline 30 & Main Jemez Spring & VA-93 & -- & -- & 1.2 & -- & - & -- & -- & -- & $-:$ & -- & -- & -- & -- & $<0.02$ & -- & $<0.02$ & -- & -- \\
\hline 30 & Main Jemez Spring & VA-122 & -- & 0.08 & 0.8 & 0.28 & -- & -- & - & -- & 0.21 & 0.21 & -- & -- & 0.001 & -- & -- & -- & -- & -- \\
\hline 30 & Ma in Jemez Spring & $V A-143$ & -- & 0.1 & 0.6 & 0.24 & -- & -- & 0.02 & -- & 0.24 & 0.39 & -- & -- & - & -- & -- & -- & 1.15 & 0.03 \\
\hline 30 & Main Jemez Spring & $V A-147$ & -- & -- & -- & - & -- & -- & -- & - & -- & - & -- & -- & -- & -- & -- & -- & -- & -- \\
\hline 30 & $\begin{array}{l}\text { Jemez Springs } \\
\text { Geotherma } 1 \text { Hell }\end{array}$ & VA-121 & -- & 0.04 & 0.8 & 0.27 & -- & -- & -- & -- & 0.13 & 0.19 & -- & -- & 0.001 & -- & -- & -- & -- & -- \\
\hline 30 & $\begin{array}{l}\text { Jemez Springs } \\
\text { Geothermal Hell }\end{array}$ & VA-144 & -- & -- & 0.07 & 0.25 & -- & -- & -- & -- & 0.17 & 0.36 & -- & 0.33 & -- & -- & -- & -- & 1.20 & - \\
\hline 30 & Jemez We11/24 m & VA-19 & $<0.03$ & 0.013 & -- & $<0.12$ & $<0.03$ & $<0.06$ & $<0.03$ & $<0.04$ & 0.39 & 0.11 & $<0.10$ & -- & $<0.05$ & -. & $<0.14$ & -- & -- & $<0.01$ \\
\hline
\end{tabular}




\begin{tabular}{|c|c|c|c|c|c|c|c|c|c|c|c|c|c|c|c|c|c|c|c|c|}
\hline $\begin{array}{l}\text { Map } \\
\text { No. }\end{array}$ & Name & $\begin{array}{l}\text { Field } \\
\text { No. }\end{array}$ & $\mathrm{Ag}$ & A) & As & $8 a$ & Cd & co & $\mathrm{Cr}$ & $\mathrm{Cu}$ & $\mathrm{Fe}$ & Mn & Mo & $\mathrm{NH}_{4}$ & $\mathrm{Ni}$ & $\mathrm{NO}_{3}$ & Pb & $\mathrm{PO}_{4}$ & $\mathrm{Rb}$ & $2 n$ \\
\hline 30 & Jemez We11/24 m & VA-25 & 0.002 & 0.030 & -- & 0.202 & $<0.03$ & 0.002 & 0.002 & 0.002 & 0.21 & 0.22 & $<0.10$ & -- & $<0.05$ & -- & 0.006 & -- & - & $<0.01$ \\
\hline 30 & Jemez We $11 / 152 \mathrm{~m}$ & VA-15 & $<0.03$ & 0.018 & -- & $<0.12$ & $<0.03$ & 0.10 & $<0.03$ & $<0.04$ & 0.39 & 0.02 & $<0.10$ & -- & 0.10 & -- & $<0.14$ & -- & -- & $<0.02$ \\
\hline 30 & Jemez Well/152 m & VA-21 & $<0.03$ & -- & -- & $<0.12$ & $<0.03$ & $<0.06$ & $<0.03$ & $<0.04$ & 1.72 & 0.07 & $<0.10$ & -- & $<0.05$ & -- & $<0.14$ & - & -- & $<0.01$ \\
\hline 30 & $\begin{array}{l}\text { Travertine Mound } \\
\text { Spring }\end{array}$ & $V A-7$ & $<0.03$ & -- & -- & $<0.12$ & 0.03 & $<0.06$ & $<0.03$ & $<0.04$ & 0.15 & 0.18 & $<0.10$ & -- & $<0.05$ & - & $<0.14$ & - & -- & 0.03 \\
\hline 30 & $\begin{array}{l}\text { Travertine Mound } \\
\text { Spring }\end{array}$ & VA-17 & $<0.03$ & 0.016 & -- & 0.20 & $<0.03$ & 0.06 & $<0.03$ & $<0.04$ & 0.15 & 0.11 & $<0.10$ & -- & 0.10 & -- & $<0.14$ & -- & - & 0.02 \\
\hline 30 & $\begin{array}{l}\text { Travertine Mound } \\
\text { Spring }\end{array}$ & VA-66 & $<0.001$ & -- & -- & 0.232 & $<0.001$ & $<0.002$ & 0.001 & $<0.001$ & 0.05 & 0.23 & $<0.002$ & -- & 0.002 & $<0.4$ & $<0.004$ & $<0.4$ & -- & $<0.002$ \\
\hline 30 & $\begin{array}{l}\text { Travertine Mound } \\
\text { Spring }\end{array}$ & VA-71 & -- & 0.009 & 0.7 & 0.111 & -- & -- & -- & -- & 0.13 & 0.19 & -- & -- & -- & $<0.1$ & -- & $<0.1$ & -- & -- \\
\hline 30 & $\begin{array}{l}\text { Travertine Mound } \\
\text { Spring }\end{array}$ & VA-91 & -- & -- & 1.1 & -- & -- & -- & -- & -- & -- & -- & -- & $\cdots$ & -- & $<0.02$ & -- & $<0.02$ & -- & -- \\
\hline 30 & $\begin{array}{l}\text { Travertine Mound } \\
\text { Spring }\end{array}$ & VA-123 & -- & 0.09 & 0.8 & 0.27 & -- & -- & -- & -- & 0.16 & 0.21 & - & -- & -- & - & -- & -- & -- & -- \\
\hline 30 & $\begin{array}{l}\text { Travertine Mound } \\
\text { Spring }\end{array}$ & $V A-142$ & -- & - & 0.6 & 0.25 & -- & -- & 0.001 & -- & 0.19 & 0.40 & -- & -- & -- & - & -- & -- & 1.15 & -- \\
\hline 30 & Buddhist Spring & $V A-8$ & $<0.03$ & - & -- & 0.60 & 0.03 & $<0.06$ & $<0.03$ & $<0.04$ & 0.18 & 0.24 & $<0.10$ & -- & 0.10 & - & $<0.14$ & -- & -- & 0.02 \\
\hline 30 & Buddhist Spring & VA-16 & $<0.03$ & 0.013 & - & $<0.12$ & $<0.03$ & $<0.06$ & $<0.03$ & $<0.04$ & 0.16 & 0.19 & $<0.10$ & -- & 0.10 & -- & $<0.14$ & -- & -- & 0.02 \\
\hline 30 & Buddhist Spring & VA-92 & -- & -- & 0.8 & -- & -- & -- & - & -- & -- & -- & -- & -- & -- & $<0.02$ & -- & $<0.02$ & -- & -- \\
\hline $\begin{array}{l}30 \\
\text { San } \\
\end{array}$ & $\begin{array}{l}\text { Unnamed Spring } \\
\text { n Ysidro - Jemez P }\end{array}$ & $\begin{array}{l}\text { VA-12 } \\
\text { b1o Area }\end{array}$ & $<0.03$ & -- & $\sim$ & 0.35 & 0.06 & 0.12 & $<0.03$ & $<0.04$ & 0.99 & 0.49 & $<0.10$ & -- & 0.10 & -- & $<0.14$ & -- & -- & 0.02 \\
\hline 36 & Ponderosa Spring & VA-39 & $<0.001$ & 0.021 & -- & 0.044 & $<0.001$ & $<0.002$ & $<0.001$ & $<0.001$ & 0.272 & 0.144 & 0.014 & -- & $<0.001$ & $<0.2$ & $<0.004$ & $<0.2$ & -- & $<0.002$ \\
\hline 36 & Ponderosa Spring & $\mathrm{VA}-135$ & - & -- & -- & 0.05 & -- & -- & - & - & 1.2 & 0.18 & - & -- & -- & - & -- & $\cdots$ & -- & -- \\
\hline 37 & Cañon Spring & $V A-40$ & $<0.001$ & 0.006 & -- & 0.082 & $<0.001$ & $<0.002$ & $<0.05$ & $<0.05$ & 0.81 & 0.148 & 0.048 & -- & $<0.03$ & 1.1 & $<0.004$ & $<0.2$ & -- & 0.016 \\
\hline 55 & $\begin{array}{l}\text { San Ysidro } \\
\text { Mineral Spring }\end{array}$ & $V A-33$ & 0.006 & 0.014 & -- & 0.044 & 0.006 & 0.016 & 0.008 & 0.009 & 3.40 & 1.80 & $<0.10$ & -- & 0.002 & -- & $<0.14$ & - & - & 0.001 \\
\hline 55 & $\begin{array}{l}\text { San Ysidro } \\
\text { Warm Spring }\end{array}$ & $V A-130$ & -- & 0.2 & 0.5 & 0.02 & -- & 0.015 & 0.007 & 0.003 & 11.4 & 1.9 & -- & -- & 0.016 & - & -- & $\cdots$ & -- & 0.013 \\
\hline
\end{tabular}


TABLE B-III (cont)

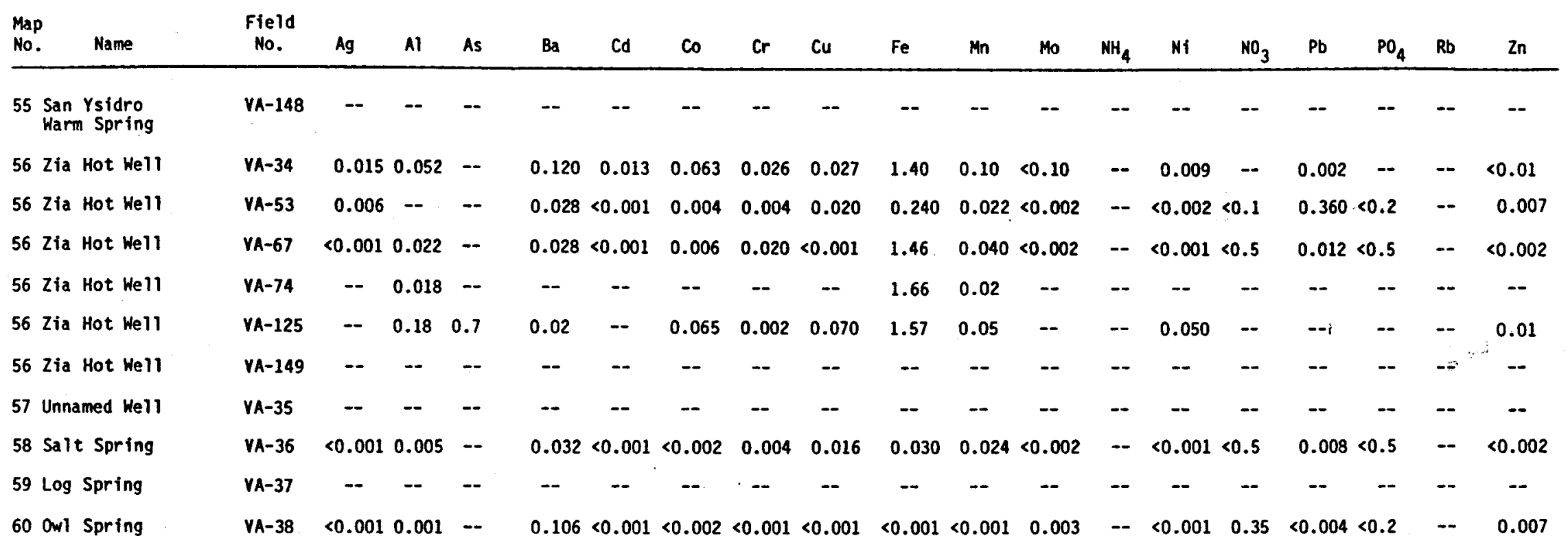


TABLE B-IV

OXYGEN AND HYDROGEN ISOTOPE ANALYSES OF WATERS IN THE JEMEZ MOUNTAINS REGION, NEW MEXICO

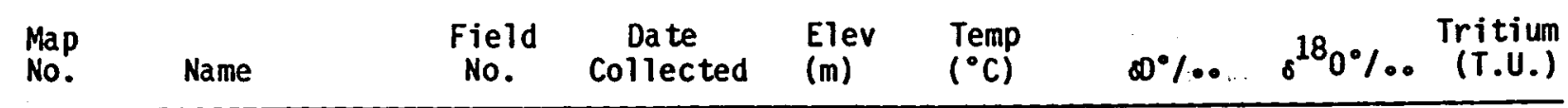

Surface and Near-Surface Meteoric Waters

\begin{tabular}{|c|c|c|c|c|c|c|c|}
\hline 1 & Gallery Spring & LA-1 & $8 / 78$ & 2439 & 11 & -84.3 & -12.20 \\
\hline 2 & T-3 Well & LA-2 & $8 / 78$ & 2018 & 13 & -73.8 & -10.65 \\
\hline 3 & $\mathrm{~T}-2 \mathrm{Well}$ & LA-3 & $8 / 78$ & 2024 & 11 & -73.5 & -10.60 \\
\hline 4 & Sacred Spring & LA-4 & $8 / 78$ & 1719 & 14 & -81.8 & -11.80 \\
\hline 5 & Basalt Spring & LA-5 & $8 / 78$ & 1835 & 15 & -76.5 & -10.85 \\
\hline 6 & L-6 We 11 & LA-6 & $9 / 78$ & 1762 & 27 & -94.7 & -13.45 \\
\hline 7 & L-1B Well & $L A-7$ & $9 / 78$ & 1716 & 30 & -103.0 & -14.30 \\
\hline 8 & L-5 Well & LA-8 & $9 / 78$ & 1785 & 26.5 & -- & -- \\
\hline 9 & L-4 Well & LA-9 & $9 / 78$ & 1847 & 28 & -- & -- \\
\hline 10 & PM-2 Well & $L A-10$ & $9 / 78$ & 2048 & 23.5 & -77.5 & -11.40 \\
\hline 11 & PM-1 Well & LA-11 & $9 / 78$ & 1981 & 28 & -74.1 & -10.95 \\
\hline 12 & G-6 We 11 & LA-12 & $9 / 78$ & 1963 & 30.5 & -76.0 & -11.25 \\
\hline 13 & G-5 We11 & LA-13 & $9 / 78$ & 1926 & 26.5 & - & -- \\
\hline 14 & G-4 Well & $L A-14$ & $9 / 78$ & 1902 & 26 & -76.3 & -11.10 \\
\hline 15 & G-3 Well & LA-15 & $9 / 78$ & 1872 & 29 & -- & -- \\
\hline 16 & G-2 Well & $L A-16$ & $9 / 78$ & 1847 & 30 & -83.1 & -11.95 \\
\hline 17 & G-1A Well & LA-17 & $9 / 78$ & 1835 & 28 & -82.5 & -11.80 \\
\hline 18 & G-1 Well & $L A-18$ & $9 / 78$ & 1823 & 29 & -81.0 & -11.65 \\
\hline 19 & $\begin{array}{l}\text { Spring, White } \\
\text { Rock Canyon }\end{array}$ & LA-19 & $9 / 78$ & 1646 & 19 & -76.8 & -11.00 \\
\hline 20 & PM-3 Well & $L A-20$ & $9 / 78$ & 2024 & 27.5 & -- & -- \\
\hline 2 & Pajarito Spring & $V A-29$ & $7 / 79$ & 1680 & 20 & -74.5 & -10.90 \\
\hline
\end{tabular}


TABLE B-IV (cont)

\begin{tabular}{|c|c|c|c|c|c|c|c|c|}
\hline $\begin{array}{l}\text { Map } \\
\text { No. }\end{array}$ & Name & $\begin{array}{l}\text { Fie1d } \\
\text { No. }\end{array}$ & $\begin{array}{l}\text { Date } \\
\text { Collected }\end{array}$ & $\begin{array}{l}\text { Elev } \\
\text { (m) }\end{array}$ & $\begin{array}{l}\text { Temp } \\
\left({ }^{\circ} \mathrm{C}\right)\end{array}$ & $80 \%$ & $\delta^{18} 0 \%$ & $\begin{array}{l}\text { Tritium } \\
\text { (T.U.) }\end{array}$ \\
\hline 33 & $\begin{array}{l}\text { Spring, White } \\
\text { Rock Canyon }\end{array}$ & $V A-30$ & $7 / 79$ & 1640 & 18 & -- & -- & -- \\
\hline 52 & $\begin{array}{l}\text { Unnamed cold } \\
\text { Spring }\end{array}$ & $V A-58$ & $7 / 80$ & 2020 & 15 & -80.5 & -11.55 & $:--$ \\
\hline 53 & $\begin{array}{l}\text { Unnamed Cold } \\
\text { Spring }\end{array}$ & VA-59 & $7 / 80$ & 2050 & 17 & -80.1 & -11.60 & -- \\
\hline 27 & San Antonio Creek & VA-104 & $9 / 82$ & 2570 & 22.4 & -- & - & 6.4 \\
\hline 28 & San Antonio Creek & $V A-24$ & $5 / 79$ & 2570 & 2 & -92.9 & -12.85 & - \\
\hline 28 & San Antonio Creek & $V A-95$ & $3 / 82$ & 2570 & 3.0 & -90.5 & -12.85 & 6.3 \\
\hline 28 & San Antonio Creek & $V A-127$ & $3 / 83$ & 2570 & 0.5 & -- & -- & 15.5 \\
\hline 29 & $\begin{array}{l}\text { Jemez River at } \\
\text { Soda Dam }\end{array}$ & VA-52 & $4 / 80$ & 1930 & 5 & -94.4 & -13.20 & -- \\
\hline 29 & $\begin{array}{l}\text { Jemez River at } \\
\text { Soda Dam }\end{array}$ & $V A-88$ & $3 / 82$ & 1930 & 1.9 & -89.5 & -12.50 & 13 \\
\hline 29 & $\begin{array}{l}\text { Jemez River at } \\
\text { Soda Dam }\end{array}$ & $V A-111$ & $1 / 83$ & 1930 & 0.7 & -- & -- & 11.8 \\
\hline 29 & $\begin{array}{l}\text { Jemez River at } \\
\text { Soda Dam }\end{array}$ & VA-131 & $5 / 83$ & 1930 & 9 & -85.4 & -12.00 & 32.6 \\
\hline 31 & Panorama Spring & $V A-28$ & $5 / 79$ & 2070 & 13 & -86.9 & -11.80 & -- \\
\hline 31 & Panorama Spring & VA-86 & $3 / 82$ & 2070 & 7.2 & -87.4 & -11.90 & 4.3 \\
\hline 31 & Panorama Spring & $V A-136$ & $5 / 83$ & 2070 & 9 & -- & -- & 1.56 \\
\hline 35 & Sino Spring & VA-32 & $8 / 79$ & 2300 & 21 & -88.0 & -12.30 & -- \\
\hline 35 & Sino Spring & VA-63 & $12 / 80$ & 2300 & 18 & -- & -- & -- \\
\hline 35 & Sino Spring & $V A-69$ & $6 / 81$ & 2300 & 22 & -- & -- & -- \\
\hline 35 & Sino Spring & $V A-85$ & $3 / 82$ & 2300 & 16.9 & -87.2 & -11.90 & 1.1 \\
\hline 35 & Sino Spring & $V A-102$ & $9 / 82$ & 2300 & 21.3 & -- & -- & 0.27 \\
\hline
\end{tabular}


TABLE B-IV (cont)

\begin{tabular}{|c|c|c|c|c|c|c|c|c|}
\hline $\begin{array}{l}\text { Map } \\
\text { No. }\end{array}$ & Name & $\begin{array}{l}\text { Field } \\
\text { No. }\end{array}$ & $\begin{array}{l}\text { Date } \\
\text { Collected }\end{array}$ & $\begin{array}{l}\text { Elev } \\
(m)\end{array}$ & $\begin{array}{l}\text { Temp } \\
\left({ }^{\circ} \mathrm{C}\right)\end{array}$ & $80 \%$ & $\delta^{18} 0 \%$ & $\begin{array}{l}\text { Tritium } \\
(T . U .)\end{array}$ \\
\hline 38 & $\begin{array}{l}\text { Indian Valley } \\
\text { Well }\end{array}$ & $V A-41$ & $\begin{array}{l}8 / 79 \\
10 / 79\end{array}$ & 2460 & $\overline{17.5}$ & $\begin{array}{l}-87.1 \\
-91.1\end{array}$ & $\begin{array}{l}-12.30 \\
-12.75\end{array}$ & -- \\
\hline 40 & Unnamed Spring & $V A-43$ & $8 / 79$ & 2770 & 14.8 & -78.2 & -11.40 & -- \\
\hline 40 & Unnamed Spring & VA-139 & $5 / 83$ & 2770 & 3 & -79.3 & -11.65 & 44.3 \\
\hline 41 & Unnamed Spring & $V A-44$ & $8 / 79$ & 2490 & 9.8 & -98.2 & -13.75 & -- \\
\hline 43 & Unnamed Spring & $V A-46$ & $8 / 79$ & 2330 & 13.3 & -96.5 & -13.60 & -- \\
\hline 44 & $\begin{array}{l}\text { Seven Springs } \\
\text { Spring }\end{array}$ & $V A-47$ & $8 / 79$ & 2480 & 12 & -99.1 & -14.25 & -- \\
\hline 44 & $\begin{array}{l}\text { Seven Springs } \\
\text { Spring }\end{array}$ & $V A-138$ & $5 / 83$ & 2480 & 12 & -96.2 & -13.50 & 20.6 \\
\hline 44 & $\begin{array}{l}\text { Cold Spring west } \\
\text { of caldera }\end{array}$ & $V A-134$ & $5 / 83$ & 2480 & -- & - & -- & -- \\
\hline 45 & $\begin{array}{l}\text { Unnamed Cold } \\
\text { Spring }\end{array}$ & $V A-48$ & $6 / 80$ & 2500 & 9 & -- & -- & -- \\
\hline 46 & Eddy's Well & $V A-49$ & $6 / 80$ & 2390 & 15 & -- & -- & -- \\
\hline 46 & Henson's Well & $V A-61$ & $12 / 80$ & 2390 & 19 & -81.2 & -11.30 & -- \\
\hline 48 & $\begin{array}{l}\text { Unnamed Cold } \\
\text { Spring }\end{array}$ & $V A-54$ & $6 / 80$ & 2500 & 11 & -88.6 & -12.60 & -- \\
\hline 49 & $\begin{array}{l}\text { Unnamed Cold } \\
\text { Spring }\end{array}$ & $V A-55$ & $6 / 80$ & $2650^{\circ}$ & 8.5 & -90.5 & -12.75 & -- \\
\hline 50 & $\begin{array}{l}\text { Unnamed Cold } \\
\text { Spring }\end{array}$ & $V A-56$ & $6 / 80$ & 2560 & 6.5 & -87.6 & -12.55 & -- \\
\hline 51 & Apache Spring & $V A-57$ & $7 / 80$ & 2530 & 9 & -85.1 & -12.25 & -- \\
\hline 54 & Turkey Spring & $V A-60$ & $7 / 80$ & 2130 & 18 & -76.0 & -11.00 & -- \\
\hline 54 & Turkey Spring & $V A-137$ & $5 / 83$ & 2130 & 18 & -75.7 & -10.90 & 1.65 \\
\hline
\end{tabular}

a Well water collected twice during same year. 
TABLE B-IV (cont)

\begin{tabular}{|c|c|c|c|c|c|c|c|c|}
\hline $\begin{array}{l}\text { Map } \\
\text { No. }\end{array}$ & Name & $\begin{array}{l}\text { Field } \\
\text { No. }\end{array}$ & $\begin{array}{l}\text { Date } \\
\text { Collected }\end{array}$ & $\begin{array}{l}\text { Elev } \\
(m)\end{array}$ & $\begin{array}{l}\text { Temp } \\
\left({ }^{\circ} \mathrm{C}\right)\end{array}$ & $\delta 0 \%$ & $\delta^{18} 0 \%$ & $\begin{array}{l}\text { Tritium } \\
\text { (T.U.) }\end{array}$ \\
\hline 68 & $\begin{array}{l}\text { Las Conchas } \\
\text { Spring }\end{array}$ & VA-124 & $1 / 83$ & 2554 & 14 & -- & -- & -- \\
\hline \multicolumn{9}{|c|}{ Valles Caldera - Sulphur Springs Area } \\
\hline 21 & $\begin{array}{l}\text { Mudpot, Men's } \\
\text { Ba thhouse }\end{array}$ & $V A-13$ & $1 / 79$ & 2500 & 78 & -50.2 & -3.25 & -- \\
\hline 21 & $\begin{array}{l}\text { Mudpot, Men's } \\
\text { Ba thhouse }\end{array}$ & $S-7-80$ & $9 / 80$ & 2500 & 82 & -- & -- & -- \\
\hline 21 & $\begin{array}{l}\text { Mudpot, Men's } \\
\text { Ba thhouse }\end{array}$ & $V A-75$ & $1 / 82$ & 2500 & 72 & - & - & 2.1 . \\
\hline 21 & $\begin{array}{l}\text { Steam, Men's } \\
\text { Ba thhouse }\end{array}$ & VA-81 & $3 / 82$ & 2500 & 13.6 & -85.1 & -12.50 & 2.3 \\
\hline 21 & Women's Bathhouse & $S-6-80$ & $9 / 80$ & 2500 & 90 & -- & -- & -- \\
\hline 21 & Women's Bathhouse & VA-76 & $1 / 82$ & 2500 & 89 & -- & -- & 19 \\
\hline 21 & Footbath Springs & $S-4-80$ & $9 / 80$ & 2500 & 33 & -- & -- & -- \\
\hline 21 & Footbath Springs & VA-77 & $1 / 82$ & 2500 & 18 & -107.0 & -23.15 & 13 \\
\hline 21 & Footbath Springs & VA-79 & $3 / 82$ & 2500 & 14.6 & -- & - & -- \\
\hline 21 & Unnamed Spring & $S-3-80$ & $9 / 80$ & 2500 & 11 & - & - & -- \\
\hline 21 & $\begin{array}{l}\text { Unnamed Hot } \\
\text { Spring }\end{array}$ & $S-9-80$ & $9 / 80$ & 2500 & -- & -- & -- & -- \\
\hline 21 & $\begin{array}{l}\text { Unnamed Hot } \\
\text { Spring }\end{array}$ & VA-14 & $1 / 79$ & 2500 & 63 & -60.7 & -8.80 & -- \\
\hline 21 & Electric Spring & $S-5-80$ & $9 / 80$ & 2500 & 36 & -- & -- & -- \\
\hline 21 & Lemonade Spring & $S-10-80$ & $9 / 80$ & 2500 & 58 & -- & -- & - \\
\hline 21 & Sulphur Creek & VA-78 & $1 / 82$ & 2500 & 0.5 & -- & -- & 39 \\
\hline 21 & $\begin{array}{l}\text { Steam, Ma in } \\
\text { Fumarole }\end{array}$ & $V A-80$ & $3 / 82$ & 2500 & 88 & - & -- & -- \\
\hline 22 & $\begin{array}{l}\text { Spring, Alamo } \\
\text { Canyon }\end{array}$ & $S-1-80$ & $9 / 80$ & 2610 & 11 & $-\infty$ & -- & -- \\
\hline
\end{tabular}


TABLE B-IV (cont)

\begin{tabular}{|c|c|c|c|c|c|c|c|c|}
\hline $\begin{array}{l}\text { Map } \\
\text { No. }\end{array}$ & Name & $\begin{array}{l}\text { Field } \\
\text { No. }\end{array}$ & $\begin{array}{l}\text { Date } \\
\text { Collected }\end{array}$ & $\begin{array}{l}\text { Elev } \\
(m)\end{array}$ & $\begin{array}{l}\text { Temp } \\
\left({ }^{\circ} \mathrm{C}\right)\end{array}$ & $\infty 0 \%$ & $\delta^{18} 0 \%$ & $\begin{array}{l}\text { Tritium } \\
\text { (T.U.) }\end{array}$ \\
\hline 22 & $\begin{array}{l}\text { Creek, Alamo } \\
\text { Canyon }\end{array}$ & $S-2-80$ & $9 / 80$ & 2610 & 11 & -- & -- & -- \\
\hline 22 & Bubbling Seep & $V A-23$ & $3 / 79$ & 2610 & 7 & - & -- & -- \\
\hline 23 & Bubbling Pool & VA-22 & $3 / 79$ & 2610 & 0.5 & -97.3 & -13.45 & -- \\
\hline 24 & $\begin{array}{l}\text { Spring, Short } \\
\text { Canyon }\end{array}$ & $S-8-80$ & $9 / 80$ & 2610 & 8 & -- & -- & -- \\
\hline 63 & $\begin{array}{l}\text { GRI Mudpit- } \\
\text { WC } \# 23-4\end{array}$ & VA-107 & $12 / 82$ & 2627 & 1.5 & -- & -- & -- \\
\hline 63 & $\begin{array}{l}\text { GRI Well at } \\
4800 \mathrm{ft} W C \# 23-4\end{array}$ & VA-113 & $1 / 83$ & 2627 & 214 & - & - & -- \\
\hline 63 & $\begin{array}{l}\text { GRI Well at } \\
4800 \mathrm{ft} \text { WC \#23-4 }\end{array}$ & VA-114 & $1 / 83$ & 2627 & - & -80.4 & -7.80 & 56.1 \\
\hline 63 & GRI Wellhead & VA-115 & $1 / 83$ & 2627 & -- & -- & -- & - \\
\hline 63 & $\begin{array}{l}\text { GRI Well at } \\
6300 \mathrm{ft} \text { WC } \# 23-4\end{array}$ & $V A-116$ & $1 / 83$ & 2627 & 232.6 & -71.5 & -5.05 & -- \\
\hline \multicolumn{9}{|c|}{ Valles Caldera - Ring Fracture Zone } \\
\hline 25 & Spence Hot Spring & $V A-1$ & $7 / 78$ & 2240 & 45 & -86.4 & -12.35 & -- \\
\hline 25 & Spence Hot Spring & $V A-68$ & $6 / 81$ & 2240 & 42 & -- & -- & -- \\
\hline 25 & Spence Hot Spring & $V A-72$ & $10 / 81$ & 2240 & 42 & -- & -- & -- \\
\hline 25 & Spence Hot Spring & VA-83 & $3 / 82$ & 2240 & 41.6 & -36.5 & -12.25 & 1.9 \\
\hline 25 & Spence Hot Spring & $V A-105$ & $9 / 82$ & 2240 & 42.5 & -- & -- & -- \\
\hline 25 & Spence Hot Spring & $V A-120$ & $1 / 83$ & 2240 & 42.3 & -- & -- & 0.20 \\
\hline 25 & $\begin{array}{l}\text { Little Spence Hot } \\
\text { Spring }\end{array}$ & VA-2 & $7 / 78$ & 2240 & 34 & -- & -- & -- \\
\hline 26 & McCauley Spring & $V A-3$ & $7 / 78$ & 2240 & 31 & -88.4 & -12.60 & -- \\
\hline 26 & McCauley Spring & $V A-87$ & $3 / 82$ & 2240 & 31.5 & -89.1 & -12.45 & 2.3 \\
\hline 26 & McCauley Spring & $V A-119$ & $1 / 83$ & 2240 & 31.9 & -- & -- & 0.27 \\
\hline
\end{tabular}


TABLE B-IV (cont)

\begin{tabular}{|c|c|c|c|c|c|c|c|c|}
\hline $\begin{array}{l}\text { Map } \\
\text { No. }\end{array}$ & Name & $\begin{array}{l}\text { Field } \\
\text { No. }\end{array}$ & $\begin{array}{l}\text { Date } \\
\text { collected }\end{array}$ & $\begin{array}{l}\text { Elev } \\
(m)\end{array}$ & $\begin{array}{l}\text { Temp } \\
\left({ }^{\circ} \mathrm{C}\right)\end{array}$ & $80 \%$ & $\delta^{18} 0 \%$ & $\begin{array}{l}\text { Tritium } \\
\text { (T.U.) }\end{array}$ \\
\hline 27 & $\begin{array}{l}\text { San Antonio Hot } \\
\text { Spring }\end{array}$ & $V A-4$ & $7 / 78$ & 2550 & 42 & -92.0 & -12.65 & -- \\
\hline 27 & $\begin{array}{l}\text { San Antonio Hot } \\
\text { Spring }\end{array}$ & $V A-96$ & $3 / 82$ & 2550 & 40.8 & -91.6 & -12.70 & 5.1 \\
\hline 27 & $\begin{array}{l}\text { San Antonio Hot } \\
\text { Spring }\end{array}$ & $V A-128$ & $3 / 83$ & 2550 & 41.3 & -- & -- & 0.35 \\
\hline 28 & Bathhouse Spring & $V A-20$ & $2 / 79$ & 2570 & 38 & -86.4 & -12.40 & -- \\
\hline 28 & Bathhouse Spring & $V A-94$ & $3 / 82$ & 2570 & 37.4 & -85.0 & -11.80 & 8.3 \\
\hline 28 & Bathhouse Spring & $V A-126$ & $3 / 83$ & 2570 & 38.1 & -- & -- & 0.44 \\
\hline 34 & Battleship Seep & $V A-31$ & $8 / 79$ & 2070 & 19 & -92.9 & -12.50 & -- \\
\hline 34 & Battleship Seep & $V A-50$ & $4 / 80$ & 2070 & 11 & -92.7 & -12.80 & -- \\
\hline 34 & Battleship Seep & $V A-133$ & $5 / 83$ & 2070 & 12 & -- & -- & 3.40 \\
\hline 34 & $\begin{array}{l}\text { East Fork Jemez } \\
\text { River }\end{array}$ & $V A-84$ & $3 / 82$ & 2070 & 4.4 & -88.0 & -12.40 & 13 \\
\hline 34 & $\begin{array}{l}\text { East Fork Jemez } \\
\text { River }\end{array}$ & $V A-106$ & $9 / 82$ & 2070 & -- & -88.0 & -12.40 & -- \\
\hline 39 & $\begin{array}{l}\text { Valle Grande } \\
\text { Spring }\end{array}$ & $V A-42$ & $8 / 79$ & 2630 & 15 & -85.0 & -12.40 & -- \\
\hline 39 & $\begin{array}{l}\text { Valle Grande } \\
\text { Spring }\end{array}$ & $V A-82$ & $3 / 82$ & 2630 & 13.6 & -85.1 & -12.50 & 2.3 \\
\hline 39 & $\begin{array}{l}\text { Valle Grande } \\
\text { Spring }\end{array}$ & $V A-117$ & $1 / 83$ & 2630 & 13.8 & -- & -- & 1.93 \\
\hline 42 & Horseshoe Spring & $V A-45$ & $8 / 79$ & 2420 & 14 & -90.2 & -12.65 & - \\
\hline 42 & Horseshoe Spring & $V A-118$ & $1 / 83$ & 2420 & 11.3 & -88.9 & -12.20 & 2.32 \\
\hline 61 & $\mathrm{PCl}$ at $225 \mathrm{ft}$ & $\mathrm{PC} 1-7$ & $10 / 83$ & 2487 & -- & - & -- & -- \\
\hline 61 & $\mathrm{PCl}$ at $365-391 \mathrm{ft}$ & $P C 1-8$ & $10 / 83$ & 2487 & -- & -- & -- & -- \\
\hline 61 & $\mathrm{PCl}$ at $685-691 \mathrm{ft}$ & PC1-9 & $10 / 83$ & 2487 & -- & -- & -- & -- \\
\hline
\end{tabular}


TABLE B-IV (cont)

\begin{tabular}{|c|c|c|c|c|c|c|c|c|}
\hline $\begin{array}{l}\text { Map } \\
\text { No. }\end{array}$ & Name & $\begin{array}{l}\text { Field } \\
\text { No. }\end{array}$ & $\begin{array}{l}\text { Date } \\
\text { Collected }\end{array}$ & $\begin{array}{l}\text { Elev } \\
(m)\end{array}$ & $\begin{array}{l}\text { Temp } \\
\left({ }^{\circ} \mathrm{C}\right)\end{array}$ & $80 \%$ & $\delta^{18} 0 \%$ & $\begin{array}{c}\text { Tritium } \\
\text { (T.U.) }\end{array}$ \\
\hline 61 & PCl at $943 \mathrm{ft}$ & PC1-10 & $10 / 83$ & 2487 & -- & -- & -- & -- \\
\hline 61 & PC1 at $1100 \mathrm{ft}$ & $P C 1-11$ & $10 / 83$ & 2487 & -- & -- & -- & -- \\
\hline 61 & $\mathrm{PCl}$ at $1712 \mathrm{ft}$ & $\mathrm{PCl}-1$ & $4 / 84$ & 2487 & -- & -- & -- & -- \\
\hline 61 & PC1 at $1937 \mathrm{ft}$ & PC1-2 & $4 / 84$ & 2487 & -- & -- & -- & -- \\
\hline 61 & PC1 at $1987 \mathrm{ft}$ & $\mathrm{PC} 1-3$ & $4 / 84$ & 2487 & -- & -- & -- & -- \\
\hline 61 & PC1 at $1953 \mathrm{ft}$ & $\mathrm{PCl}-4$ & $4 / 84$ & 2487 & -- & -- & -- & -- \\
\hline 61 & PC1 at $2036 \mathrm{ft}$ & $\mathrm{PC} 1-5$ & $5 / 84$ & 2487 & -- & -- & -- & -- \\
\hline 71 & PC2 at $587-600$ & ft PC2-1 & $8 / 84$ & 2627 & 30 & -- & -- & -- \\
\hline 71 & $P C 2$ at $630-635$ & ft PC2-2 & $8 / 84$ & 2627 & 30 & -- & - & -- \\
\hline 71 & PC2 at $1086 \mathrm{ft}$ & PC2-3 & $9 / 84$ & 2627 & -- & -- & -- & -- \\
\hline 71 & PC2 at $1350 \mathrm{ft}$ & PC2-4 & $9 / 84$ & 2627 & 35 & -- & - & -- \\
\hline 71 & PC2 at $1360 \mathrm{ft}$ & $P C 2-5$ & $9 / 84$ & 2627 & 38 & -- & -- & -- \\
\hline 71 & PC2 at $1335 \mathrm{ft}$ & PC2-6 & $9 / 84$ & 2627 & 40 & - & - & -- \\
\hline 71 & PC2 at $1490 \mathrm{ft}$ & PC2-7 & $10 / 84$ & 2627 & 41 & -- & -- & -- \\
\hline$\frac{\text { Valle }}{62}$ & $\begin{array}{l}\text { s Caldera - Baca } \\
\text { Redondo Creek } \\
\text { at Union Gate }\end{array}$ & $\frac{\text { Geothermal }}{\sqrt{A-129}}$ & $\frac{1 \text { Field }}{5 / 83}$ & 2390 & 4 & -88.7 & -12.50 & 34.1 \\
\hline 64 & Baca Well \#4 & $B A-2$ & $6 / 11 / 82$ & 2835 & 294 & -86.5 & -9.20 & -- \\
\hline 64 & Baca Well \#4 & $B A-5$ & $7 / 2 / 82$ & 2835 & 297 & -86.0 & -9.25 & 0.49 \\
\hline 65 & Baca Well \#13 & BA-1 & $6 / 4 / 82$ & 2841 & 278 & -97.0 & -9.30 & -- \\
\hline 65 & Baca Well \#13 & $B A-4$ & $7 / 1 / 82$ & 2841 & 279 & -85.0 & -9.20 & 0.61 \\
\hline 66 & Baca Well \#15 & BA-7 & $7 / 23 / 82$ & 2774 & 267 & -82.5 & -8.00 & 0.25 \\
\hline 66 & Baca Well \#15 & $B A-8$ & $9 / 7 / 82$ & 2774 & 326 & -85.0 & -8.20 & 0.18 \\
\hline 66 & Baca Well \#19 & $B A-9$ & $10 / 15 / 82$ & 2774 & 223 & -80.0 & -7.60 & 0.47 \\
\hline 67 & Baca Well \#24 & $B A-3$ & $6 / 18 / 82$ & 2661 & 260 & -83.5 & -8.00 & 1.10 \\
\hline 67 & Baca Well \#24 & $B A-6$ & $7 / 16-82$ & 2661 & 261 & -83.5 & -8.15 & 0.71 \\
\hline
\end{tabular}


TABLE B-IV (cont)

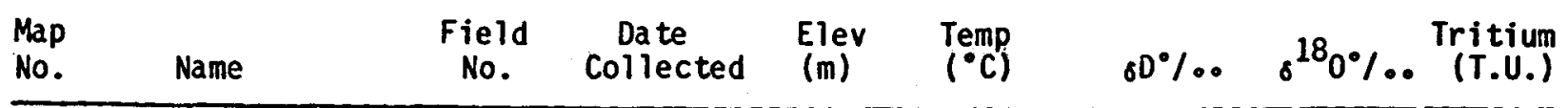

Valles Caldera - Soda Dam and Jemez Springs Area

\begin{tabular}{|c|c|c|c|c|c|c|c|c|}
\hline 29 & Soda Dam Spring & $V A-6$ & $7 / 78$ & 1930 & 47 & -84.9 & -10.60 & - \\
\hline 29 & Soda Dam Spring & $V A-9$ & $1 / 79$ & 1930 & 48 & -- & -- & -- \\
\hline 29 & Soda Dam Spring & $V A-26$ & $5 / 79$ & 1930 & 47 & -- & -- & -- \\
\hline 29 & Soda Dam Spring & $V A-51$ & $4 / 80$ & 1930 & 47 & -85.4 & -10.70 & -- \\
\hline 29 & Soda Dam Spring & $V A-64$ & $12 / 80$ & 1930 & 47 & -85.2 & -10.60 & -- \\
\hline 29 & Soda Dam Spring & VA-70 & $6 / 81$ & 1930 & 72 & -- & -- & -- \\
\hline 29 & Soda Dam Spring & $V A-73$ & $10 / 81$ & 1930 & 53 & -- & -- & -- \\
\hline 29 & Soda Dam Spring & VA-89 & $3 / 82$ & 1930 & 47 & -- & -- & 2.9 \\
\hline 29 & Soda Dam Spring & VA-99 & $9 / 82$ & 1930 & 47.5 & -- & - & 1.33 \\
\hline 29 & Soda Dam Spring & VA-109 & $1 / 83$ & 1930 & 46.8 & -84.0 & -10.35 & 1.48 \\
\hline 29 & Soda Dam Spring & VA-132 & $5 / 83$ & 1930 & 47 & -- & - & 1.62 \\
\hline 29 & Soda Dam Spring & VA-140 & $2 / 84$ & 1930 & 46.8 & -- & -- & -- \\
\hline 29 & Soda Dam Spring & VA-146 & $4 / 84$ & 1930 & -- & - & -- & -- \\
\hline 29 & Grotto Spring & VA-5 & $7 / 78$ & 1930 & 38 & -84.6 & -10.65 & -- \\
\hline 29 & $\begin{array}{l}\text { Outfal1 of Soda } \\
\text { Dam Spring }\end{array}$ & VA-65 & $12 / 80$ & 1930 & 17 & -- & -- & -- \\
\hline 29 & $\begin{array}{l}\text { Hidden Warm } \\
\text { Spring }\end{array}$ & $V A-27$ & $5 / 79$ & 1930 & 29 & -84.9 & -10.95 & -- \\
\hline 29 & $\begin{array}{l}\text { Hidden Warm } \\
\text { Spring }\end{array}$ & VA-90 & $3 / 82$ & 1930 & 32 & -85.1 & -10.65 & 5.7 \\
\hline 29 & $\begin{array}{l}\text { Hidden Warm } \\
\text { Spring }\end{array}$ & $V A-110$ & $1 / 83$ & 1930 & 32.3 & -- & -- & 3.63 \\
\hline 29 & $\begin{array}{l}\text { Hidden Warm } \\
\text { Spring }\end{array}$ & $V A-141$ & $2 / 84$ & 1930 & 32.2 & -- & -- & -- \\
\hline 30 & Main Jemez Spring & $V A-10$ & $1 / 79$ & 1890 & 55 & -82.3 & -10.6 & -- \\
\hline 30 & Main Jemez Spring & $V A-18$ & $1 / 79$ & 1890 & 36 & -81.4 & -10.4 & -- \\
\hline
\end{tabular}




\section{TABLE B-IV (cont)}

\begin{tabular}{|c|c|c|c|c|c|c|c|c|}
\hline $\begin{array}{l}\text { Map } \\
\text { No. }\end{array}$ & Name & $\begin{array}{l}\text { Field } \\
\text { No. }\end{array}$ & $\begin{array}{l}\text { Date } \\
\text { Collected }\end{array}$ & $\begin{array}{l}\text { Elev } \\
(m)\end{array}$ & $\begin{array}{l}\text { Temp } \\
\left({ }^{\circ} \mathrm{C}\right)\end{array}$ & $80 \%$ & $\delta^{18} 0 \%$ & $\begin{array}{l}\text { Tritium } \\
\text { (T.U.) }\end{array}$ \\
\hline 30 & Main Jemez Spring & VA-93 & $3 / 82$ & 1890 & 46.3 & -82.1 & -10.40 & 1.2 \\
\hline 30 & Ma in Jemez Spring & $V A-122$ & $1 / 83$ & 1890 & 74.9 & -- & -- & -- \\
\hline 30 & Main Jemez Spring & VA-143 & $2 / 84$ & 1890 & 74.7 & -- & -- & -- \\
\hline 30 & Main Jemez Spring & VA-147 & $4 / 84$ & 1890 & -- & -- & -- & -- \\
\hline 30 & $\begin{array}{l}\text { Jemez Springs } \\
\text { Geothermal We11 }\end{array}$ & $V A-121$ & $1 / 83$ & 1890 & 74.9 & -- & -- & -- \\
\hline 30 & $\begin{array}{l}\text { Jemez Springs } \\
\text { Geothermal We11 }\end{array}$ & $V A-144$ & $2 / 84$ & 1890 & 72.2 & -- & -- & -- \\
\hline 30 & Jemez We11/24 m & $V A-19$ & $1 / 79$ & 1890 & 68 & -84.0 & -11.3 & -- \\
\hline 30 & Jemez We11/24 m & $V A-25$ & $5 / 79$ & 1890 & 73.3 & -- & -- & -- \\
\hline 30 & Jemez We11/152 m & $V A-15$ & $1 / 79$ & 1890 & 60.5 & -85.9 & -11.8 & -- \\
\hline 30 & Jemez We11/152 m & $V A-21$ & $2 / 79$ & 1890 & 61 & -- & -- & -- \\
\hline 30 & $\begin{array}{l}\text { Travertine Mound } \\
\text { Spring }\end{array}$ & VA-7 & $1 / 79$ & 1890 & 70 & -83.6 & -11.30 & -- \\
\hline 30 & $\begin{array}{l}\text { Travertine Mound } \\
\text { Spring }\end{array}$ & $V A-17$ & $1 / 79$ & 1890 & 72 & -- & -- & -- \\
\hline 30 & $\begin{array}{l}\text { Travertine Mound } \\
\text { Spring }\end{array}$ & VA-66 & $12 / 80$ & 1890 & 72 & -83.1 & -11.35 & -- \\
\hline 30 & $\begin{array}{l}\text { Travertine Mound } \\
\text { Spring }\end{array}$ & $V A-71$ & $6 / 81$ & 1890 & 72 & -- & -- & -- \\
\hline 30 & $\begin{array}{l}\text { Travertine Mound } \\
\text { Spring }\end{array}$ & $V A-91$ & $3 / 82$ & 1890 & 72.3 & -- & -- & 6.7 \\
\hline 30 & $\begin{array}{l}\text { Travertine Mound } \\
\text { Spring }\end{array}$ & VA-123 & $1 / 83$ & 1890 & 72.6 & -- & -- & 2.92 \\
\hline 30 & $\begin{array}{l}\text { Travertine Mound } \\
\text { Spring }\end{array}$ & $V A-142$ & $2 / 84$ & 1890 & 72.9 & -- & -- & -- \\
\hline 30 & Buddnist Spring & $V A-8$ & $1 / 79$ & 1890 & 49 & -- & -- & -- \\
\hline 30 & Buddhist Spring & $V A-16$ & $1 / 79$ & 1890 & 50 & -- & -- & -- \\
\hline 30 & Buddhist Spring & VA-92 & $3 / 82$ & 1890 & 43.2 & -83.6 & -11.05 & 10 \\
\hline
\end{tabular}


TABLE B-IV (cont)

\begin{tabular}{|c|c|c|c|c|c|c|c|c|}
\hline $\begin{array}{l}\text { Map } \\
\text { No. }\end{array}$ & Name & $\begin{array}{l}\text { Field } \\
\text { No. }\end{array}$ & $\begin{array}{l}\text { Date } \\
\text { Collected }\end{array}$ & $\begin{array}{l}\text { Elev } \\
(m)\end{array}$ & $\begin{array}{l}\text { Temp } \\
\left({ }^{\circ} \mathrm{C}\right)\end{array}$ & $80 \%$ & $\delta^{18} 0 \%$ & $\begin{array}{l}\text { Tritium } \\
\text { (T.U.) }\end{array}$ \\
\hline 30 & Unnamed Spring & $V A-12$ & $1 / 79$ & 1890 & 73.3 & -- & -- & -- \\
\hline \multicolumn{9}{|c|}{ San Ysidro - Jemez Pueblo Area } \\
\hline 36 & Ponderosa Spring & VA-39 & $8 / 79$ & 1740 & 16.8 & -84.6 & -12.35 & -- \\
\hline 36 & Ponderosa Spring & VA-135 & $5 / 83$ & 1740 & 16.8 & -- & -- & 2.07 \\
\hline 37 & Cañon Spring & $V A-40$ & $8 / 79$ & 1830 & 20.2 & -86.6 & -12.35 & -- \\
\hline 55 & $\begin{array}{l}\text { San Ysidro } \\
\text { Mineral Spring }\end{array}$ & $V A-33$ & $8 / 79$ & 1680 & 27 & -86.6 & -10.40 & -- \\
\hline 55 & $\begin{array}{l}\text { San Ysidro } \\
\text { Warm Spring }\end{array}$ & VA-130 & $5 / 83$ & 1680 & 22 & -- & -- & 0.40 \\
\hline 55 & $\begin{array}{l}\text { San Ysidro } \\
\text { Warm Spring }\end{array}$ & VA-148 & $4 / 84$ & 1680 & -- & -- & -- & -- \\
\hline 56 & Zia Hot Well & $V A-34$ & $8 / 79$ & 1840 & 56 & -89.8 & -11.25 & -- \\
\hline 56 & Zia Hot Well & $V A-53$ & $4 / 80$ & 1840 & 54 & -89.0 & -12.55 & - \\
\hline 56 & Zia Hot Well & $V A-67$ & $3 / 81$ & 1840 & 53 & -- & -- & -- \\
\hline 56 & Zia Hot Well & $V A-74$ & $10 / 81$ & 1840 & 53 & -- & -- & -- \\
\hline 56 & Zia Hot Well & VA-125 & $2 / 83$ & 1840 & 53.0 & -- & -- & 0.05 \\
\hline 56 & Zia Hot Well & VA-149 & $4 / 84$ & 1840 & -- & - & -- & - \\
\hline 57 & Unnamed Well & $V A-35$ & $8 / 79$ & 1920 & 21 & -101.4 & -13.65 & -- \\
\hline 58 & Salt Spring & $V A-36$ & $8 / 79$ & 1690 & 29.2 & -84.9 & -10.20 & - \\
\hline 59 & Log Spring & $V A-37$ & $8 / 79$ & 2190 & 28.5 & -87.7 & -12.25 & -- \\
\hline 60 & Owl Spring & $V A-38$ & $8 / 79$ & 1760 & 17.6 & -86.2 & -12.15 & -- \\
\hline
\end{tabular}


GAS ANALYSES FROM VALLES CALDERA REGION IN VOLUME PERCENTAGES

\begin{tabular}{|c|c|c|c|c|c|c|c|c|c|c|c|}
\hline & & & & & Iphur Sprin & gs Area & & & & & \\
\hline & $\begin{array}{l}\text { Men's } \\
\text { Bathhouse } \\
\text { Spring }\end{array}$ & $\begin{array}{l}\text { Men's } \\
\text { Bathhouse } \\
\text { Spring }\end{array}$ & $\begin{array}{l}\text { Fumarole by } \\
\text { Men's } \\
\text { Bathhouse }\end{array}$ & $\begin{array}{l}\text { Women's } \\
\text { Bathhouse } \\
\text { Spring }\end{array}$ & $\begin{array}{l}\text { Women's } \\
\text { Bathhouse } \\
\text { Spring }\end{array}$ & $\begin{array}{l}\text { Footbath } \\
\text { Spring }\end{array}$ & $\begin{array}{l}\text { Footbath } \\
\text { Spring }\end{array}$ & $\begin{array}{l}\text { Spring } \\
\text { Alamo } \\
\text { Canyon }\end{array}$ & $\begin{array}{c}\text { Alamo Canyon } \\
\text { Gas Seep }\end{array}$ & $\begin{array}{l}\text { Skin } \\
\text { Spring }\end{array}$ & $\begin{array}{c}\text { Lemonade } \\
\text { Spring }\end{array}$ \\
\hline $\begin{array}{l}\text { Sample } \\
\text { Date }\end{array}$ & $\begin{array}{c}S-7-81 \\
8 / 81\end{array}$ & $\begin{array}{c}\text { VA-154 } \\
6 / 84\end{array}$ & $\begin{array}{c}S-12-81^{\mathrm{a}} \\
8 / 81\end{array}$ & $\begin{array}{l}V A-97 \\
5 / 82\end{array}$ & $\begin{array}{c}\text { VA-155 } \\
6 / 84\end{array}$ & $\begin{array}{c}S-4-80 \\
9 / 80\end{array}$ & $\begin{array}{r}V A-98 \\
5 / 82\end{array}$ & $\begin{array}{l}S-1-80 \\
9 / 80\end{array}$ & $\begin{array}{c}S-1-81 \\
8 / 81\end{array}$ & $\begin{array}{c}S-5-80 \\
9 / 80\end{array}$ & $\begin{array}{c}S-6-80^{2} \\
9 / 80\end{array}$ \\
\hline $\mathrm{CO}_{2}$ & 97.0 & 96.79 & 60.1 & 96.93 & 96.90 & 71.9 & 99.28 & 94.5 & 96.2 & 92.3 & 79.4 \\
\hline $\mathrm{H}_{2} \mathrm{~S}$ & 0.5 & 2.10 & 0.3 & 1.25 & 1.89 & 0.31 & 0.60 & 0.53 & 0.5 & 0.38 & 0.67 \\
\hline $\mathrm{NH}_{3}$ & - & -- & -- & -- & - & -- & -- & -- & -- & -- & -- \\
\hline $\mathrm{H}_{2}$ & -- & 0.303 & - & 0.180 & 0.376 & -- & 0.300 & -- & -- & -- & -- \\
\hline He & -- & 0.076 & -- & $<0.005$ & -- & -- & $<0.005$ & -- & -- & -- & -- \\
\hline$N_{2}$ & 1.8 & 1.15 & 31.9 & 1.41 & 0.168 & 23.2 & 0.14 & 4.2 & 2.5 & 6.0 & 16.1 \\
\hline $0_{2}$ & 0.7 & -- & 7.7 & 0.16 & -- & 4.6 & $<0.01$ & 0.8 & 0.8 & 1.4 & 3.8 \\
\hline Ar & -- & -- & -- & 0.02 & -- & -- & $<0.01$ & -- & -- & -- & -- \\
\hline $\mathrm{CH}_{4}$ & -- & -- & -- & 0.020 & -- & -- & 0.030 & -- & -- & -- & -- \\
\hline $\mathrm{C}_{2} \mathrm{H}_{6}$ & -- & - & -- & $<0.01$ & -- & -- & $<0.01$ & -- & -- & -- & -- \\
\hline Total & 100.00 & 100.42 & 100.00 & 99.99 & 99.334 & 100.01 & 100.38 & 100.03 & 100.00 & 100.08 & 99.97 \\
\hline
\end{tabular}

\section{Empirical Gas Geothermometers}

\begin{tabular}{|c|c|c|c|c|c|c|c|c|c|c|c|}
\hline $\mathrm{CH}_{4}-\mathrm{H}_{2}^{\mathrm{b}}$ & -- & -- & $\cdots$ & 215 & -- & -- & 215 & -- & -- & -- & -- \\
\hline $\mathrm{CO}_{2}-\mathrm{CH}_{4}{ }^{\mathrm{C}}$ & -- & -- & -- & 287 & -- & -- & 273 & -- & -- & -- & - \\
\hline
\end{tabular}


TABLE B-V (cont)

Sulphur Springs Area (cont)

\begin{tabular}{|c|c|c|c|c|c|c|c|c|c|c|c|c|}
\hline & $\begin{array}{l}\text { Short } \\
\text { Canyon }\end{array}$ & $\begin{array}{l}\text { Short } \\
\text { Canyon }\end{array}$ & $\begin{array}{l}\text { Fumarole } \\
\text { Spring }\end{array}$ & $\begin{array}{l}\text { Electric } \\
\text { Spring }\end{array}$ & $\begin{array}{l}\text { Sulphur } \\
\text { Creek }\end{array}$ & $\begin{array}{l}\text { GRI } \\
\text { Mudpit }\end{array}$ & $\begin{array}{l}\text { GRI } \\
\text { Hel1 }\end{array}$ & $\begin{array}{l}\text { GRI Well } \\
\text { at } 4800 \mathrm{ft}\end{array}$ & $\begin{array}{l}\text { GRI } \\
\text { WeII }\end{array}$ & $\begin{array}{l}\text { GRI Well at } \\
\text { Wellhead }\end{array}$ & $\begin{array}{l}\text { Mudpot, } \\
\text { Bathho }\end{array}$ & $\begin{array}{l}\text { len's } \\
\text { ise }\end{array}$ \\
\hline $\begin{array}{l}\text { Sample } \\
\text { Date }\end{array}$ & $\begin{array}{l}5-8-80 \\
9 / 80\end{array}$ & $\begin{array}{c}5-8-81 \\
8 / 81\end{array}$ & $\begin{array}{c}S-10-80 \\
9 / 80\end{array}$ & $\begin{array}{c}S-5-81 \\
8 / 81\end{array}$ & $\begin{array}{l}S-11-81 \\
8 / 81\end{array}$ & $\begin{array}{l}\text { VA-107 } \\
12 / 82\end{array}$ & $\begin{array}{l}\text { VA-108 } \\
12 / 82\end{array}$ & $\begin{array}{c}V A-113 \\
1 / 83\end{array}$ & $\begin{array}{c}V A-114 \\
1 / 83\end{array}$ & $\begin{array}{c}V A-115 \\
1 / 83\end{array}$ & $\begin{array}{c}V A-75 a^{2} \\
1 / 82\end{array}$ & $\begin{array}{r}V A-75 \\
1 / 82\end{array}$ \\
\hline $\mathrm{CO}_{2}$ & 95.7 & 94.0 & 95.7 & 97.0 & 96.6 & 98.0 & 98.78 & 90.36 & 99.0 & 82.68 & 26.34 & 88.24 \\
\hline $\mathrm{H}_{2} \mathrm{~S}$ & 0.61 & 0.5 & 0.61 & 0.3 & 0.3 & -- & $<0.02$ & 0.071 & 0.17 & $<0.01$ & 0.22 & 0.78 \\
\hline $\mathrm{NH}_{3}$ & -- & -- & -- & -- & -- & -- & -- & 0.062 & 0.03 & -- & -- & -- \\
\hline $\mathrm{H}_{2}$ & -- & - & -- & - & -- & 0.46 & 0.89 & $\cdots$ & 0.02 & 0.95 & 0.05 & 0.195 \\
\hline $\mathrm{He}$ & -- & -- & -- & -- & -- & -- & 0.005 & -- & 0.001 & $<0.005$ & $<0.005$ & 0.005 \\
\hline $\mathrm{N}_{2}$ & 3.7 & 4.4 & 3.7 & 2.1 & 2.5 & 1.34 & 0.01 & 7.38 & 0.62 & 12.28 & 57.66 & 8.45 \\
\hline $\mathrm{O}_{2}$ & -- & 1.1 & -- & 0.6 & 0.6 & 0.20 & $<0.01$ & 1.72 & 0.10 & 3.17 & 15.3 & 2.05 \\
\hline$A r$ & - & - & -- & -- & -- & - & 0.45 & - & 0 & 0.14 & 0.70 & 0.10 \\
\hline $\mathrm{CH}_{4}$ & -- & -- & -- & -- & -- & -- & $<0.01$ & -- & 0 & $<0.002$ & 0.005 & 0.020 \\
\hline $\mathrm{C}_{2} \mathrm{H}_{6}$ & -- & -- & -- & -- & -- & -- & $<0.01$ & -- & 0 & $<0.001$ & $<0.01$ & $<0.01$ \\
\hline Total & 100.01 & 100.00 & 100.01 & 100.00 & 100.00 & 100.00 & 100.18 & 99.60 & 99.94 & 99.24 & 100.29 & 99.85 \\
\hline
\end{tabular}

Empirical Gas Geothermometers

$\mathrm{CO}_{2}-\mathrm{H}_{2} \mathrm{~S}$

\begin{tabular}{|c|c|c|c|c|c|c|c|c|c|c|}
\hline $\mathrm{CH}_{4}-\mathrm{H}_{2}^{\mathrm{b}}$ & -- & -- & -- & -- & -- & 209 & -- & -- & 221 & 288 \\
\hline $\mathrm{O}_{2}-\mathrm{CH}_{4}{ }^{\mathrm{C}}$ & -- & -- & -- & - & -- & 313 & -- & -- & 365 & 290 \\
\hline
\end{tabular}




\section{Buca Geothernol Field}

\begin{tabular}{|c|c|c|c|c|c|c|c|c|c|c|c|c|c|c|c|c|c|c|c|c|}
\hline \multirow[t]{2}{*}{ Sample } & \multicolumn{3}{|c|}{ Bace 4} & \multicolumn{3}{|c|}{ Baca 13} & \multicolumn{3}{|c|}{ Baca 13} & \multirow{2}{*}{$\frac{\text { Raca } 15}{\text { Titration }}$} & \multirow{2}{*}{$\frac{\text { Baca } 15}{\text { Titration }}$} & \multicolumn{3}{|c|}{ Baca 19} & \multicolumn{3}{|c|}{ Baca 24} & \multicolumn{3}{|c|}{ Baca 24} \\
\hline & $\begin{array}{l}\text { Grav. } \\
\text { Anal. }\end{array}$ & $\begin{array}{c}\text { Titra- } \\
\text { tion }\end{array}$ & Average & $\begin{array}{l}\text { Grav. } \\
\text { Anal. }\end{array}$ & $\begin{array}{c}\text { Titra- } \\
\text { tion }\end{array}$ & Average & $\begin{array}{l}\text { Grav. } \\
\text { Anal. }\end{array}$ & $\begin{array}{c}\text { Titra- } \\
\text { tion }\end{array}$ & Average & & & $\begin{array}{l}\text { Grey. } \\
\text { Anel. }\end{array}$ & $\begin{array}{c}\text { Titra- } \\
\text { tion }\end{array}$ & Average & $\begin{array}{l}\text { Grav. } \\
\text { Anti. }\end{array}$ & $\begin{array}{c}\text { Titra- } \\
\text { tion }\end{array}$ & Average & $\begin{array}{l}\text { Grav. } \\
\text { Anai. }\end{array}$ & $\begin{array}{c}\text { Titra- } \\
\text { tion }\end{array}$ & Average \\
\hline DATE & \multicolumn{3}{|c|}{$7 / 2 / 82$} & \multicolumn{3}{|c|}{$6 / 4 / 82$} & \multicolumn{3}{|c|}{$7 / 1 / 82$} & $7 / 23 / 82$ & 9/8/82 & \multicolumn{3}{|c|}{$11 / 12 / 82$} & \multicolumn{3}{|c|}{$6 / 18 / 82$} & \multicolumn{3}{|c|}{$7 / 16 / 82$} \\
\hline $\mathrm{CO}_{2}$ & 98.44 & 98.70 & 98.57 & 99.28 & 99.36 & 99.32 & 99.15 & 99.31 & 99.23 & 99.34 & 95.49 & 98.38 & 97.95 & 98.165 & 96.48 & 98.75 & 98.615 & 98.4 & 98.82 & 98.61 \\
\hline $\mathrm{H}_{2} \mathrm{~S}$ & 0.995 & 0.792 & 0.894 & 0.482 & 0.408 & 0.445 & 0.534 & 0.382 & 0.458 & 0.295 & 0 & 0.806 & 1.28 & 1.043 & 0.672 & 0.394 & 0.533 & 0.778 & 0.371 & 0.575 \\
\hline $\mathrm{NH}_{3}$ & 0.027 & 0.024 & 0.026 & 0.017 & 0.017 & 0.017 & 0.017 & 0.017 & 0.017 & 0.015 & 0.847 & 0.049 & 0.047 & 0.048 & 0.042 & 0.042 & 0.042 & 0.096 & 0.094 & 0.095 \\
\hline$H_{2}$ & 0.07 & 0.063 & 0.067 & 0.004 & 0.039 & 0.04 & 0.052 & 0.05 & 0.051 & 0.034 & 0.223 & 0.107 & 0.101 & 0.104 & 0.054 & 0.054 & 0.054 & 0.032 & 0.032 & 0.032 \\
\hline HE & 0.007 & 0.007 & 0.007 & 0.004 & 0.004 & 0.004 & 0.006 & 0.005 & 0.006 & 0.005 & 0.004 & 0.006 & 0.006 & 0.006 & 0.002 & 0.002 & 0.002 & 0.002 & 0.002 & 0.002 \\
\hline$N_{2}$ & 0.381 & 0.345 & 0.363 & 0.142 & 0.138 & 0.140 & 0.189 & 0.183 & 0.186 & 0.273 & 0.290 & 0.581 & 0.550 & 0.566 & 0.721 & 0.721 & 0.721 & 0.679 & 0.668 & 0.674 \\
\hline $0_{2}$ & 0 & 0 & 0 & 0 & 0 & 0 & 0 & 0 & 0 & 0 & 0 & 0 & 0 & 0 & 0 & 0 & 0 & 0 & 0 & 0 \\
\hline$A R$ & 0.01 & 0.009 & 0.01 & 0.005 & 0.004 & 0.005 & 0.007 & 0.006 & 0.007 & 0.007 & 0.078 & 0.017 & 0.016 & 0.017 & 0.018 & 0.018 & 0.018 & 0.013 & 0.013 & 0.013 \\
\hline $\mathrm{CH}_{4}$ & 0.042 & 0.038 & 0.04 & 0.021 & 0.020 & 0.021 & 0.026 & 0.025 & 0.026 & 0.006 & 0.015 & 0.064 & 0.061 & 0.063 & 0.018 & 0.015 & 0.018 & 0.009 & 0.008 & 0.009 \\
\hline $\mathrm{C}_{2} \mathrm{H}_{6}$ & 0 & 0 & 0 & 0 & 0 & 0 & 0 & 0 & 0 & 0 & 0 & 0 & 0 & 0 & 0 & 0 & 0 & 0 & 0 & 0 \\
\hline TOTAL & 99.97 & 99.98 & 99.977 & 99.95 & 99.99 & 99.99 & 99.98 & 99.98 & 99.981 & 99.98 & 96.95 & 100.01 & 100.01 & 100.012 & 100.01 & 100.00 & 100.005 & 100.01 & 100.01 & 100.01 \\
\hline STEAM/C & & & 120.5 & & & 67.63 & & & 63.72 & 55.75 & 122.8 & & & 193.3 & & & 154.7 & & & 185.8 \\
\hline \multicolumn{21}{|c|}{ Empirical Gas Geothermometers } \\
\hline $\mathrm{CO}_{2}-\mathrm{H}_{2}$ & $\mathrm{~S}-\mathrm{CH}_{4}-1$ & & 182 & -- & - & 169 & -- & - & 142 & 170 & -- & -- & - & 191 & -- & - & 178 & -- & -- & 173 \\
\hline $\mathrm{CO}_{2}-\mathrm{CH}_{4}$ & & & 263 & -- & -- & 288 & -- & -- & 279 & 332 & 297 & -- & $-\cdot$ & 246 & - & - & 292 & -- & -- & 317 \\
\hline
\end{tabular}


TABLE B-V (cont)

\begin{tabular}{|c|c|c|c|c|c|c|c|c|}
\hline \multirow[b]{2}{*}{ Sample } & \multicolumn{3}{|c|}{ Fenton $\mathrm{HIII}$} & \multicolumn{2}{|c|}{ San Ysidro Area } & \multicolumn{2}{|c|}{ Soda Dan and Jemez } & \multirow{2}{*}{$\begin{array}{c}\text { Springs Area } \\
\text { Battleship } \\
\text { Gas Seep } \\
\text { VA-175 }\end{array}$} \\
\hline & V-39, EE-3 & $V-40, E E-3$ & $V-42, E E-3$ & $\begin{array}{c}\text { San Ysidro } \\
\text { Warm Spring } \\
\text { YA-148 }\end{array}$ & $\begin{array}{c}\text { Zia Hot } \\
\text { Wel1 } \\
\text { VA-149 }\end{array}$ & $\begin{array}{l}\text { Main Jemez } \\
\text { Spring } \\
\text { YA-147 }\end{array}$ & $\begin{array}{l}\text { Soda Dan } \\
\text { Spring } \\
\text { VA-146 }\end{array}$ & \\
\hline Date & $4 / 7 / 83$ & $4 / 7 / 83$ & $4 / 7 / 83$ & $4 / 5 / 84$ & $4 / 5 / 84$ & $4 / 84$ & $4 / 84$ & $8 / 84$ \\
\hline $\mathrm{CO}_{2}$ & 98.72 & 99.99 & 94.36 & 95.296 & 86.147 & 93.053 & 97.474 & 96.07 \\
\hline $\mathrm{H}_{2} \mathrm{~S}$ & $<0.01$ & $<0.01$ & $<0.01$ & -- & -- & -- & $159 \mathrm{ppm}$ & 0.43 \\
\hline $\mathrm{NH}_{3}$ & -- & - & -- & -- & -- & -- & - & -- \\
\hline $\mathrm{H}_{2}$ & 0.015 & 0.005 & 0.005 & -- & -- & 0.280 & - & $<0.005$ \\
\hline He & 0.22 & 0.060 & 0.075 & 0.750 & 0.965 & 0.747 & -- & $<0.005$ \\
\hline $\mathrm{N}_{2}$ & 1.41 & 0.17 & 4.74 & 4.417 & 11.042 & 4.853 & 2.433 & 2.61 \\
\hline $\mathrm{O}_{2}$ & $<0.01$ & $<0.01$ & 1.15 & 0.091 & 0.023 & 0.220 & 0.072 & 0.66 \\
\hline Ar & 0.02 & $<0.01$ & 0.05 & $\cdots$ & 0.54 & -- & -- & 0.02 \\
\hline $\mathrm{CH}_{4}$ & $<0.002$ & $<0.002$ & $<0.002$ & -- & -- & 1.466 & -- & 0.054 \\
\hline $\mathrm{C}_{2} \mathrm{H}_{6}$ & $<0.01$ & $<0.01$ & $<0.01$ & -- & -- & -- & - & $<0.01$ \\
\hline Total & 100.42 & 100.27 & 100.40 & 100.55 & 98.72 & 100.62 & 99.98 & 99.86 \\
\hline
\end{tabular}

\section{Empirical Gas Geothermoneters}

$\mathrm{CO}_{2}-\mathrm{H}_{2} \mathrm{~S}-$

$\begin{array}{cccccccc}\mathrm{CH}_{4}-\mathrm{H}_{2}{ }^{\mathrm{b}} 129 & 111 & 112 & -- & -- & -- & -- & - \\ \mathrm{CO}_{2}-\mathrm{CH}_{4}{ }^{\mathrm{C}}{ }^{\mathrm{N}} 371 & 372 & 370 & -- & -- & 131 & -- & 251\end{array}$

a Large air contamination.

b D'Amore and Panicht (1980).

c Morman and Bernhardt (1981). 
TABLE B-VI

GEOTHERMOMETERS IN ${ }^{\circ} \mathrm{C}$

\begin{tabular}{|c|c|c|c|c|c|c|c|c|c|}
\hline \multirow[b]{2}{*}{$\begin{array}{l}\text { Field } \\
\text { No. }\end{array}$} & \multirow[b]{2}{*}{ Name } & \multirow[b]{2}{*}{$\begin{array}{l}\text { Mea sured } \\
\text { Temp }\end{array}$} & \multicolumn{2}{|r|}{$\mathrm{SiO}_{2}$} & \multirow[b]{2}{*}{$\mathrm{Na}-\mathrm{K}$} & \multicolumn{3}{|c|}{$\mathrm{Na}-\mathrm{K}-\mathrm{Ca}$} & \multirow[b]{2}{*}{$\mathrm{Na}-\mathrm{Li}^{\mathrm{b}}$} \\
\hline & & & $Q t^{a}$ & Chalcedony & & $B=4 / 3$ & $\beta=1 / 3$ & $\begin{array}{l}\text { Mg } \\
\text { Corr }\end{array}$ & \\
\hline Valles & Caldera - Baca & Geothermal F & ield & & & & & & \\
\hline$B A-2$ & Baca Well \#4 & 294 & 288 & 290 & 274 & -- & 290 & 290 & 301 \\
\hline$B A-5$ & Baca Well \#4 & 297 & 294 & 297 & 273 & -- & 288 & 288 & 298 \\
\hline BA-1 & Baca Well \#13 & 278 & 276 & 275 & 264 & -- & 282 & 282 & 330 \\
\hline$B A-4$ & Baca Well 13 & 279 & 282 & 282 & 264 & -- & 279 & 279 & 303 \\
\hline$B A-7$ & Baca Well \#15 & 267 & 282 & 282 & 267 & -- & 272 & 272 & 287 \\
\hline BA-8 & Baca Well \#15 & 326 & -- & -- & 275 & -- & 277 & 277 & 297 \\
\hline BA-9 & Baca Well \#19 & 223 & 264 & 259 & 262 & -- & 269 & 269 & 303 \\
\hline BA-3 & Baca Well \#24 & 260 & 273 & 271 & 235 & -- & 244 & 244 & 293 \\
\hline$B A-6$ & Baca Well \#24 & 261 & 270 & 267 & 238 & -- & 247 & 247 & 295 \\
\hline AVERAGE & & 277 & 279 & 278 & 261 & -- & 272 & 272 & 301 \\
\hline \multicolumn{10}{|c|}{ Sulphur Springs Area - GRI Well } \\
\hline VA-113 & $\begin{array}{l}\text { GRI Well at } \\
4800 \mathrm{ft}\end{array}$ & 214 & 232 & 220 & 266 & - & 258 & 257 & 265 \\
\hline VA-116 & $\begin{array}{l}\text { GRI Well at } \\
6300 \mathrm{ft}\end{array}$ & 232.6 & 243 & 234 & 269 & -- & 287 & 287 & 283 \\
\hline
\end{tabular}


TABLE B-VI (cont)

\begin{tabular}{|c|c|c|c|c|c|c|c|c|c|}
\hline \multirow[b]{2}{*}{$\begin{array}{l}\text { Field } \\
\text { No. }\end{array}$} & \multirow[b]{2}{*}{ Name } & \multirow[b]{2}{*}{$\begin{array}{l}\text { Mea sured } \\
\text { Temp }\end{array}$} & \multicolumn{2}{|c|}{$\mathrm{SiO}_{2}$} & \multirow[b]{2}{*}{$\mathrm{Na}-\mathrm{K}$} & \multicolumn{3}{|c|}{$\mathrm{Na}-\mathrm{K}-\mathrm{Ca}$} & \multirow[b]{2}{*}{$\mathrm{Na}-\mathrm{Li}$} \\
\hline & & & $\mathbf{Q t z}^{\mathrm{a}}$ & Cha rcedony & & $\beta=4 / 3$ & $B=1 / 3$ & $\begin{array}{l}\text { Mg } \\
\text { Corr }\end{array}$ & \\
\hline \multirow[t]{3}{*}{ Soda $D$} & $n$ and Jemez Spring & Is Area & & & & & & & \\
\hline & $\begin{array}{l}\text { Soda Dam Spring } \\
\text { (Avg. of } 10 \text { ) }\end{array}$ & 47 & 98 & 68 & 274 & 175 & 222 & 167 & 317 \\
\hline & $\begin{array}{l}\text { Hidden Warm Spr. } \\
\text { (Avg. of } 2 \text { ) }\end{array}$ & 32.2 & 95 & 65 & 261 & 166 & 213 & 165 & 306 \\
\hline \multirow[t]{4}{*}{$V A-5$} & Grotto Spring & 38 & 87 & 59 & 270 & 178 & 221 & 157 & 302 \\
\hline & $\begin{array}{l}\text { Ma in Jemez Spr. } \\
(\text { Avg. of } 4)\end{array}$ & 74.8 & 131 & 104 & 221 & 153 & 191 & 175 & 305 \\
\hline & $\begin{array}{l}\text { Travertine Mound } \\
\text { (Avg. of } 6 \text { ) }\end{array}$ & 72 & 132 & 104 & 228 & 150 & 193 & 178 & 309 \\
\hline & $\begin{array}{l}\text { Buddhist Spring } \\
\text { (Avg. of 2) }\end{array}$ & 50 & 123 & 95 & 231 & 138 & 190 & 156 & 314 \\
\hline \multirow[t]{7}{*}{$V A-12$} & Unnamed Spring & 49 & 137 & 111 & 229 & 155 & 195 & 161 & 304 \\
\hline & $\begin{array}{l}\text { 80-ft Aquifer } \\
\text { at Jemez Spring } \\
\text { (Avg. of 2) }\end{array}$ & 70.7 & 121 & 93 & 227 & 146 & 191 & 175 & 303 \\
\hline & $\begin{array}{l}500-\mathrm{ft} \text { Aquifer } \\
\text { at Jemez Spring } \\
\text { (Avg. of 2) }\end{array}$ & 61 & 79 & 48 & 269 & 107 & 196 & 143 & 324 \\
\hline & Jemez Spr. Well & 72 & 133 & 105 & 219 & 150 & 189 & 175 & 288 \\
\hline & $\begin{array}{l}\text { Battleship Seep } \\
\text { (Avg. of } 3 \text { ) }\end{array}$ & 14 & 58 & 25 & 174 & 200 & 178 & 178 & 185 \\
\hline & $\begin{array}{l}\text { Ponderosa Spr. } \\
\text { (Avg. of 2) }\end{array}$ & 13 & 54 & 21 & 198 & 149 & 178 & 92 & 185 \\
\hline & $\begin{array}{l}\text { Average of } P C 1 \\
\text { Well data }\end{array}$ & -- & $144^{C}$ & $119^{c}$ & $176^{c}$ & $183^{d}$ & $183^{d}$ & $152^{C}$ & $84^{d}$ \\
\hline$P C 1-6$ & $\begin{array}{l}\text { PC1 Well; with } \\
\text { highest } \mathrm{Cl} \\
\text { content }\end{array}$ & - & 87 & 56 & $<80$ & 452 & 484 & 484 & $459^{e}$ \\
\hline
\end{tabular}


TABLE B-VI (cont)

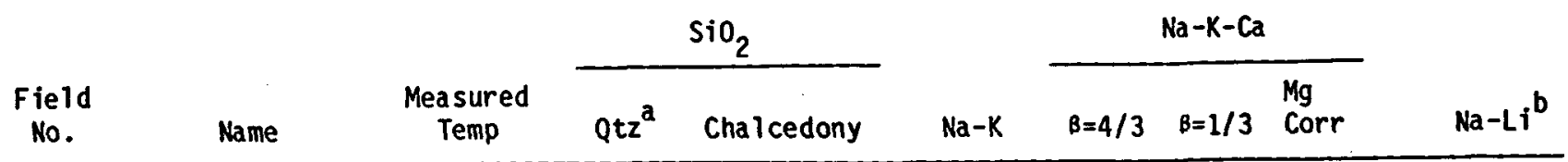

\section{Ring Fracture Zone}

Spence Hot Spr.

42.8

116

83

$129 \quad 54$

11254

304

McCauley Spr.

31.5

106

77

160

$29 \quad 118$

29

290

(Avg. of 2)

San Antonio

41.4

123

95

200

$69 \quad 152$

69

137

(Avg, of 3)

Bathhouse Spr.

38.9

138

111

256

82

181

82

144

(Avg. of 3)

San Ysidro - Jemez Pueblo Area

$\begin{array}{llllllllll}\text { VA-130 } & \begin{array}{l}\text { San Ysidro } \\ \text { Warm Spring }\end{array} & 25 & 56 & 23 & 157 & 148 & 156 & 66 & 184 \\ \begin{array}{l}\text { Zia Hot Wel1 } \\ \text { (Avg. of 4) }\end{array} & 54 & 83 & 52 & 115 & 150 & 132 & 50 & 117 \\ \text { VA-36 } & \text { Salt Spring } & 15.5 & -- & -- & 162 & 205 & 172 & 72 & 165\end{array}$

assuming no steam loss.

bquation for $\mathrm{Cl} \leq 7000 \mathrm{mg} / \mathrm{l}$ (Fouillac and Michard 1981).

${ }^{c}$ Average of 5 values.

Average of 4 values.

Equation for $\mathrm{Cl}>7000 \mathrm{mg} / \mathrm{\ell}$ (Fouillac and Michard 1981). 


\section{REFERENCES}

Aldrich, M. J., Jr., and A. W. Laughlin, "A Model for the Tectonic Development of the Southeastern Colorado Plateau Boundary," J. Geophys. Res. 89, 10, 207-218 (1984).

Bailey, R. A., R. L. Smith, and C. S. Ross, "Stratigraphic Nomenclature of Volcanic Rocks in the Jemez Mountains, New Mexico," US Geol. Surv. Bull. 1274-P, 19 pp. (1969).

Barnes, R. A., "The Determination of Specific Forms of Aluminum in Natural Water," Chem. Geol. 15, 177-191 (1975).

Charles, R. W., R. J. Vidale Buden, and F. Goff, "An Interpretation of the Alteration Assemblages at Sulphur Springs, Valles Caldera, New Mexico," J. Geophys. Res. 91(B2), 1887-1898 (1986).

Craig, P. H., "Isotopic Variations in Meteoric Waters," Science 13., 1702-1703 (1961).

D'Amore, F., and C. Panichi, "Evolution of Deep Temperatures of Hydrothermal Systems by a New Gas Geothermometer," Geochim. Cosmochim. Acta 44, 549-556 $(1980)$.

Doel1, R. R., G. B. Dalrymple, R. L. Smith, and R. A. Bailey, "Paleomagnetism, Potassium-Argon Ages, and Geology of Rhyolites and Associated Rocks of the valles Caldera, New Mexico," Geol. Soc. Am. Mem. 116, 211-248 (1968).

Fouillac, C., and G. Michard, "Sodium/Lithium Ratio in Water Applied to Geothermometry of Geothermal Reservoirs," Geothermics 10, 55-70 (1981).

Gardner, J. N., and F. Goff, "Potassium-Argon Dates from the Jemez Volcanic Field: Implications for Tectonic Activity in the North-Central Rio Grande Rift," New Mexico Geol. Soc. Guidebook, 35th Field Conf., pp. 75-81 (1984).

Gardner, J. N., F. Goff, S. Garcia, and R. C. Hagan, "Stratigraphic Relations and Lithologic Variations in the Jemez Volcanic Field, New Mexico," J. Geophys. Res. 91(B2), 1763-1778 (1986).

Goff, F. E., and S. Sayer, "A Geothermal Investigation of Spring and Well Waters of the Los Alamos Region, New Mexico," Los Alamos Scientific Laboratory report LA-8326-MS (Apri1 1980).

Goff, F. E., C. 0. Grigsby, P. E. Trujillo, Jr., D. Counce, and A. Kron, "Geology, Water Geochemistry and Geothermal Potential of the Jemez Springs Area, Cañon de San Diego, New Mexico," J. Volcanol. Geotherm. Res. 10, 227-244 (1981).

Goff, F. E., and C. 0. Grigsby, "Valles Caldera Geothermal Systems, New Mexico, USA," J. Hydro1. 56, 119-136 (1982). 
Goff, F., T. McCormick, P. Trujillo, Jr., D. Counce, and C. Grigsby, "Geochemical Data for 95 Thermal and Nonthermal Waters of Valles Caldera--Southern Jemez Mountains Region, New Mexico," Los Alamos National Laboratory report LA-9367-OBES (1982).

Goff, F. E., et a1., "Geochemistry and Isotopes of Fluids from Sulphur Springs, Valles Caldera, New Mexico," J. Volcanol. Geotherm. Res. 23, 273-297 (1985).

Griggs, R. L., "Geology and Ground-Water Resources of the Los Alamos Area, New Mexico," US Geol. Surv. Water-Supply Paper 1753, 107 pp. (1964).

Grigsby, C. 0., F. Goff, P. E. Trujillo, and D. Counce, "Geochemical Behavior of a Hot Dry Rock Geothermal Reservoir," New Mexico Geol. Soc. Guidebook, 35 th Field Conf., pp. 265-270 (1984).

Heiken, G., and F. Goff, "Hot Dry Rock Geothermal Energy in the Jemez Volcanic Field, New Mexico," J. Volcanol. Geotherm. Res. 15, 223-246 (1983).

Heiken, G., F. Goff, J. Stix, S. Tamanyu, M. Shafiqullah, S. Garcia, and R. Hagan, "Intracaldera Volcanic Activity, Toledo Caldera and Embayment, Jemez Mountains, New Mexico," J. Geophys. Res. 91(B2), 1799-1815 (1986).

Laughlin, A. W., "The Geothermal System of the Jemez Mountains and Its Exploration," in Geothermal Systems-Principles and Case Histories, $L$. Rybach and L. J. P. MuffTer, Eds. (John Witey and Sons, Inc., New York, 1981) pp. 295-320.

Laughlin, A. W., A. C. Eddy, R. Laney, and M. J. Aldrich, Jr., "Geology of the Fenton Hill, New Mexico, Hot Dry Rock Site," J. Volcanol. Geotherm. Res. 15, 21-41 (1983).

Mariner, T. R., T. S. Presser, and W. C. Evans, "Chemical, Isotopic, and Gas Compositions of Selected Thermal Springs in Arizona, New Mexico and Utah," US Geol. Surv. Open-file report 77-654, 42 pp. (1977).

Nielson, D. L., and J. B. Hulen, "Internal Geology and Evolution of the Redondo Dome, Valles Caldera, New Mexico," J. Geophys. Res. 89, 8695-8711 (1984).

Norman, D. I., and C. A. Bernhardt, "Assessment of Geothermal Reservoirs by Analysis of Gases in Thermal Waters," Final Technical Report, New Mexico Energy Institute, New Mexico State Univ., Las Cruces, 130 pp. (1981).

Phillips, F. M., F. Goff, F. Vuataz, H. W. Bentley, D. Elmore, and H. E. Grove, ${ }^{36} \mathrm{Cl}$ as a Tracer in Geothermal Systems: Example from Valles Caldera, New Mexico," Geophys. Res. Lett. 11, 1227-1230 (1984).

Purtymun, W. D., and J. B. Cooper, "Development of Ground-Water Supplies on the Pajarito Plateau," US Geo1. Surv. Prof. Paper 650-B, B149-B153 (1969).

Purtymun, W. D., and S. Johansen, "General Geohydrology of the Pajarito Plateau," New Mexico Geol. Soc. Guidebook, 25 th Field Conf., pp. 347-349 (1974). 
Purtymun, W. D., F. G. West, and W. H. Adams, "Preliminary Study of the Quality of Water in the Drainage Area of the Jemez River and Rio Guadalupe," Los Alamos Scientific Laboratory report LA-5595-MS (Apri) 1974).

Purtymun, W. D., "Hydrologic Characteristics of the Los Alamos Well Field, with Reference to the Occurrence of Arsenic in We11 L-6," Los Alamos Scientific Laboratory report LA-7012-MS (November 1977).

Purtymun, W. D., "Water Supply at Los Alamos During 1978," Los Alamos Scientific Laboratory report LA-8074-PR (October 1979).

Purtymun, W. D., R. J. Peters, and J. W. Owens, "Geohydrology of White Rock Canyon of the Rio Grande from Otowi to Frijoles Canyon," Los Alamos Scientific Laboratory report LA-8635-MS (December 1980).

Ross, C. S., R. L. Smith, and R. A. Bailey, "Outline of the Geology of the Jemez Mountains, New Mexico," New Mexico Geol. Soc. Guidebook, 12th Field Conf., pp. 139-143 (1961).

Self, S., F. Goff, J. N. Gardner, J. V. Wright, and W. M. Kite, "Explosive Rhyolitic Volcanism in the Jemez Mountains, New Mexico: Vent Locations, Caldera Development, and Relation to Regional Structure," $\mathrm{J}$. Geophys. Res. 91(B2), 1779-1798 (1986).

Smith, R. L., R. A. Bailey, and C. S. Ross, "Structural Evolution of the Valles Caldera, New Mexico, and Its Bearing on the Emplacement of Ring Dikes," US Ge01. Surv. Prof. Paper 424-D, D145-D149 (1961).

Smith, R. L., R. A. Bailey, and C. S. Ross, "Geologic Map of the Jemez Mountains, New Mexico," US Geol. Surv. Misc. Geol. Investig. Map I-571 (1970).

Summers, W. K., "Catalog of Thermal Waters in New Mexico," New Mexico Bur. Mines Miner. Resour. Hydrol. Rep. 4, 80 pp. (1976).

Titus, F. B., Jr., "Ground-Water Geology of the Rio Grande Trough in NorthCentral New Mexico, with Sections on the Jemez Caldera and the Lucero Uplift," New Mexico Geol. Soc. Guidebook, 12th Field Conf., pp. 186-192 (1961).

Trainer, F. W., "Ground-Water in the Southern Part of the Jemez Mountains Volcanic Region, New Mexico," New Mexico Geol. Soc. Guidebook, 25th Field Conf., pp. 337-345 (1974).

Trainer, F. W., "Mixing of Thermal and Nonthermal Waters in the Margin of the Rio Grande Rift, Jemez Mountains, New Mexico," New Mexico Geol. Soc. Guidebook, 26 th Field Conf., pp. 213-218 (1975).

Trainer, F. W., "Geohydrologic Data From the Jemez Mountains and Vicinity, North-Centra1 New Mexico," US Geol. Surv. Water-Resour. Invest. 77-131, $146 \mathrm{pp}$. (1978). 
Trainer, F. W., and F. P. Lyford, "Geothermal Hydrology in the Rio Grande Rift, North Central New Mexico," New Mexico Geol. Soc. Guidebook, 30th Field Conf., pp. 299-306 (1979).

Trainer, F. W., "Thermal Springs in Cañon de San Diego as a Window into Valles Caldera, New Mexico," New Mexico Geol. Soc. Guidebook, 35th Field Conf., pp. 249-255 (1984).

Truesdell, A. H., and C. J. Janik, "Reservoir Processes and Fluid Origins in the Baca Geothermal System, Valles Caldera, New Mexico," J. Geophys. Res. 91(B2), 1817-1834 (1986).

Truji110, P. E., Jr., D. Counce, C. 0. Grigsby, F. Goff, and L. Shevene11, "Chemical Analysis and Sampling Techniques for Geothermal Fluids and Gases at the Fenton Hill Laboratory," Los Alamos National Laboratory report (in prep.)

Vuataz, F. D., and F. E. Goff, "Isotope Geochemistry of Thermal Waters in the Valles Caldera, Jemez Mountains, Northern New Mexico," J. Geophys. Res. 91(B2), 1835-1853 (1986).

Whi.te, A. F., et al., "Fluid Chemistry of the Baca Geothermal Field, Valles Caldera, New Mexico," New Mexico Geol. Soc. Guidebook, 35th Field Conf., pp. 257-263 (1984).

White, D. E., "Magmatic, Connate, and Metamorphic Waters," Geol. Soc. Am. Bu11. 68, 1659-1682 (1957):

White, D. E., L. J. P. Muffler, and A. H. Truesde11, "Vapor-Dominated Hydrotherma1 Systems Compared with Hot-Water Systems," Econ. Geol. 66, 75-97 (1971).

White, A. F., "Chemical and Isotopic Characteristics of Fluids Within the Baca Geothermal Reservoir, Valles Caldera, New Mexico," J. Geophys. Res. 91(B2), 1855-1866 (1986). 